\title{
Investigations on Dynamics and Control of a Rimless Wheel based 2D Dynamic Walker using Pulsed Torque Actuation
}

\author{
A Thesis Submitted for the \\ DEGREE OF
}

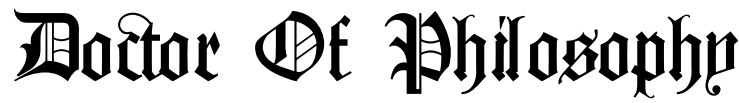

\author{
IN THE \\ FACUlty OF EngineERING
}

BY

\section{LALIT PATNAIK}

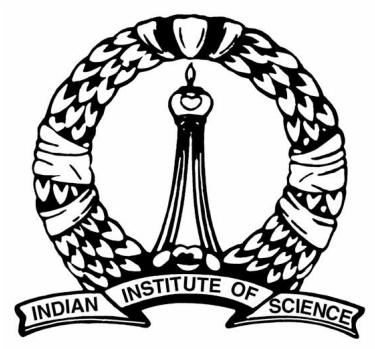

Department of Electronic Systems Engineering

Indian Institute of Science, Bangalore

NOVEMBER 2014

COPYRIGHT (C) 2014 IISC

All Rights Reserved 

Dedicated to my parents... 



\section{SYNOPSIS}

Wheeled systems are energy efficient on prepared surfaces like roads and tracks. Legged systems are capable of traversing different terrains but can be lossy. At low speeds and on off-road surfaces, legged systems using dynamic walking can be efficient. Towards this objective, the dynamics of the walker needs to be modelled and controlled. This thesis presents analysis and experiments on the dynamics and control of a rimless wheel based mobile robot (Chatur) in a category between wheeled and legged systems. It is effectively a 2D dynamic walker that serves as a platform for investigating inverted pendulum walking. A pulsed actuation torque is proposed for the system resulting in four torque regimes defined by the ratio of energy losses to available actuator torque. Five physical constraints that limit the choice of operating points of a generic inverted pendulum walker are expounded and location of optimal operating points is discussed. Chatur's hardware design is elaborated and a control topology is proposed for pulsed actuation of the dual brushless de (BLDC) motor driven platform with wheel synchronization.

A closed-form analytical solution, using hyperbolic functions, is proposed for the stance phase inverted pendulum dynamics. Suitable models for ground impact and braking losses are used. There are four types of sub-phases within a stance phase - actuated rise, unactuated rise, actuated fall, unactuated fall. These are concatenated in four different ways to form repeating cycles yielding the four torque regimes that are identified by the fraction of stance phase for which the actuator is energized. The experimental set-up is a fixed step-angle walker built using two adjacent rimless wheels independently actuated at the hub. Varying the magnitude and duty ratio of the torque pulse, the proposed regimes are experimentally shown. The mechanical cost of transport is computed for different speeds. Videos of the walks are also taken.

The operating point of an inverted pendulum walker can be specified by the combination of initial mid-stance velocity and step angle chosen for a given walk. Using basic mechanics, a framework of physical constraints that defines allowable operating points is presented. For any given speed, the optimal operating point with minimum mechanical power requirement is located based on tangency of the power and velocity contours. Repeating for different speeds, a family of optimal operating points, called the optimal locus, is obtained. Using an appropriate constant step angle over a range of speeds could lead to an inverted pendulum walker that is close to optimal.

The hardware design for Chatur and the caveats associated with reliable performance of its mechanical and electrical subsystems are elaborated. In order to ensure lateral stability, the system uses two contralateral wheels driven by separate BLDC hub motors. From a motor drive perspective, the reflected load torque has a characteristic cyclic variation that repeats several times within a mechanical revolution. The proposed control topology has two hierarchical levels, an inner loop for torque control of BLDC motor implemented using a standard proportional-integral controller, and an outer loop for torque reference generation that uses the information on the ground impact instants and the motor position feedback. Ground impacts of the spokes are detected by an accelerometer to initiate the application of torque. Wheel synchronization is achieved by incrementing the torque of the motor that is lagging. The BLDC motor drive uses non-commutating current feedback to reduce current spikes during sector transitions. Experimental waveforms for controller validation are shown. 



\section{ACKNOWLEDGMENTS}

This work would not have been possible without the able guidance of my research advisor Prof L Umanand. His insightful inputs and encouraging attitude formed the foundation and scaffolding of my work. I especially treasure the freedom he gave me in driving my research the way I wanted. I am grateful to our Chairman Prof K Gopakumar for his support and for the excellent lab facilities. The courses offered by Prof Gunasekaran, Prof NS Dinesh, Prof Rudra Pratap, Prof G Narayanan and late Prof VT Ranganathan helped me open up my engineering mind in many ways. I express my gratitude to Dr. JE Diwakar and Prof Ashitava Ghosal for their valuable inputs.

I cannot thank my lab-mates enough for the innumerable discussions and especially for creating a lab environment conducive for research with a tinge of fun. Thank you Tanmay, Das, Chintan, Rijil, Prasanna, Deepak, Rex, Abhijit, Sudharshan, Matthew, Jaison, Manoj, Sijo and others. I owe Sudharshan and Abhijit a treat for critically reviewing this thesis and suggesting several corrections.

No work of engineering can be accomplished without the right tools and the skills to use them. I thank the staff at the workshop (Murali and Sreeramappa) and PCB lab (Vasantha and Antonisamy) whose excellent skills helped me in building the experimental set-up. I sincerely appreciate the open source community for creating and supporting the free software tools that I used: gEDA/pcb, ngspice, octave, git, Freeplane, JabRef, LaTeX etc. The leap from Windows to Linux has been liberating.

No words can do justice in conveying my gratitude to my family. My parents have whole-heartedly supported me with unstinting love and patience. I dedicate this thesis to them. Special thanks to my friends circle - Pratibha, Varun, Alok, Gayatri and others in the Punter gang - for being there and for all the fun times that made life easier. I'll always cherish the times I spent with the IISc cricket team. I learnt as much in the play ground as I did in the lab. 



\section{Abstract}

Wheeled systems are energy efficient on prepared surfaces like roads and tracks. Legged systems are capable of traversing different terrains but can be lossy. At low speeds and on off-road surfaces, legged systems using dynamic walking can be energy efficient. Towards this objective, the dynamics of the walker needs to be modelled and controlled. In addition, the braking and ground impact losses need to be minimized. This thesis presents analysis and experiments on the dynamics and control of a rimless-spoked-wheel based mobile robot $\left(\right.$ Chatur $\left.^{*}\right)$ that belongs to a category between wheeled and legged systems. This rolling rimless wheel is effectively a 2D dynamic walker that serves as a platform for investigating the dynamics and energetics of inverted pendulum walking with constant step angle. A pulsed actuation torque is proposed for the system resulting in four torque regimes defined by the ratio of energy losses to available actuator torque. Five physical constraints that impose fundamental limits on the choice of operating points of a generic inverted pendulum walker are expounded and a method for locating optimal operating points is discussed. Chatur's hardware design is elaborated and a control topology is proposed for pulsed actuation of the dual brushless de (BLDC) motor driven platform with wheel synchronization.

Various actuator torque profiles can be used to achieve dynamic 'walking' in a hubactuated rimless wheel. The proposed pulsed actuation torque gives rise to four torque regimes that achieve sustained walking and a fifth regime where the walker keeps slowing down with each step. The regimes can be identified based on the fraction of stance phase for which the actuator is energized. Theoretical analysis and experimental results are presented. A simple closed-form analytical solution, using hyperbolic functions, is proposed for the stance phase inverted pendulum dynamics considering planar motion. Ground impacts are assumed to cause abrupt drop in velocity. A constant braking torque that lumps together the effect of several loss phenomena is also considered. Based on whether the CoM is rising or falling and whether or not there is an actuating torque, a stance phase can have four types of sub-phases - actuated rise, unactuated rise, actuated fall, unactuated fall. These are concatenated in four different ways to form repeating cycles yielding the four regimes. The experimental set-up is a fixed step-angle walker constructed using two synchronized adjacent rimless wheels independently actuated at the hub. Varying the magnitude and duty ratio of the torque pulse, the four proposed regimes are experimentally shown. The mechanical power consumption and cost of transport are computed from measured motor currents for different average forward speeds. Videos of the walks are also taken.

\footnotetext{
*means clever in Hindi
} 
The space of operating points for an inverted pendulum based bipedal dynamic walker in terms of constraints and optimality is investigated. The operating point of the walker can be specified by the combination of initial mid-stance velocity $\left(v_{0}\right)$ and step angle $\left(\phi_{m}\right)$ chosen for a given walk. Not all operating points lead to a realizable steady-state gait. Using basic mechanics, a framework of physical constraints that limit the choice of operating points is proposed. The constraint lines thus obtained delimit the valid region of operation of the walker in the $v_{0}-\phi_{m}$ plane. Within this allowable region, sub-regions that result in various regimes of walking are identified. A given average forward velocity $v_{x, a v g}$ can be achieved by several combinations of $v_{0}$ and $\phi_{m}$. Only one of these combinations results in the minimum mechanical power consumption and can be considered the optimum operating point for the given $v_{x, \text { avg }}$. A method is proposed for obtaining this optimal operating point based on tangency of the power and velocity contours. Putting together all such operating points for various $v_{x, a v g}$, a family of optimum operating points, called the optimal locus, is obtained. For the energy loss and internal energy models chosen, the optimal locus obtained has a largely constant step angle with increasing speed but tapers off at non-dimensional speeds close to unity. Thus, choosing the right step angle and keeping it fixed over a broad range of speeds could lead to an inverted pendulum walker that is close to optimal from a mechanical energy perspective.

The complete hardware design for Chatur and the caveats associated with reliable performance of the mechanical and electrical subsystems are elaborated. In order to ensure lateral stability, the system uses two contralateral wheels each driven by a separate BLDC hub motor. From a motor drive perspective, the mechanical load belongs to a unique class of dynamic loads whose reflected torque has a characteristic cyclic variation that repeats several times within a mechanical revolution. The proposed control topology has two hierarchical levels, an inner loop for torque control of BLDC motor implemented using a standard proportional-integral controller, and an outer loop for torque reference generation that uses the information on the ground impact instants and the motor position feedback. Ground impacts of the spokes are detected by an accelerometer to initiate the application of torque. The torque pulse magnitude can be set internally or by a manual operator via radio control. The pulse duration is programmable and enables attainment of various torque regimes at different steady state speeds. The wheels are synchronized so that corresponding spokes on both wheels move in unison. This is achieved by including a wheel synchronization loop that compensates for any lag between the wheels. Lag is detected based on number of sector changes in the hall-effect position sensor data received from both motors. An improved BLDC motor drive is developed wherein non-commutating current feedback is used to reduce current spikes during sector transitions. Experimental waveforms for controller validation are shown. 


\section{Contents}

Table of Contents $\quad$ xi

List of Figures $\quad$ Xv

List of Tables $\quad$ xix

List of Symbols $\quad$ xxi

List of Abbreviations $\quad$ xxv

1 Introduction 1

1.1 Terrestrial locomotion alternatives . . . . . . . . . . . . . . . . 1

1.2 Energetics of locomotion . . . . . . . . . . . . . . . . 2

1.3 Gaits in legged locomotion . . . . . . . . . . . . . . . . . 5 5

1.3.1 Definition of gait . . . . . . . . . . . . . . 5

1.3.2 Classification of gaits . . . . . . . . . . . . . . 5

Static walking . . . . . . . . . . . . . . . . . 7

ZMP-based walking . . . . . . . . . . . . . 8

Dynamic walking . . . . . . . . . . . . . . . 9

Choosing a locomotion alternative . . . . . . . . . . . . 11

1.3.3 Description of gaits . . . . . . . . . . . . . . . 11

1.4 Models of walking . . . . . . . . . . . . . . . . . . . . . . . 13

1.4.1 Inverted pendulum model . . . . . . . . . . . . . . . . 13

1.4.2 The rimless wheel . . . . . . . . . . . . . . . . 15

1.4 .3 Other models . . . . . . . . . . . . . . . . . . 17

1.5 Thesis scope . . . . . . . . . . . . . . . . . . . . . 17

1.6 Thesis outline . . . . . . . . . . . . . . . . . . . . . . 18 
2 Modeling of Dynamics and Torque Regimes

2.1 Passive walking on level ground . . . . . . . . . . . . . . . . 21

2.1 .1 Ideal case . . . . . . . . . . . . . . . . . . 21

2.1.2 Practical case ...................... 24

Braking loss . . . . . . . . . . . . . . . . . . 24

Impact loss . . . . . . . . . . . . . . . . . . . . . . 25

2.2 Sustained forward walk . . . . . . . . . . . . . . . . . . . 28

2.2.1 Downward incline . . . . . . . . . . . . . . . . . 28

2.2 .2 Torque actuation . . . . . . . . . . . . . . . . . 28

2.3 Analytical solution for the dynamics . . . . . . . . . . . . . 30

2.4 Torque regimes . . . . . . . . . . . . . . . . . . . . . 32

2.5 Experimental prototype . . . . . . . . . . . . . . 40

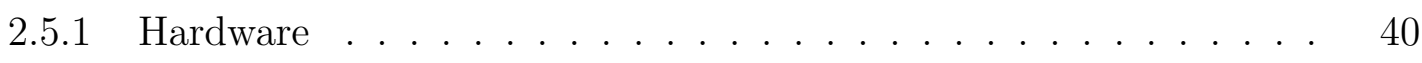

2.5.2 Control scheme ....................... 41

2.6 Experimental results . . . . . . . . . . . . . . . . . . . . . . 42

2.6.1 Torque Regimes . . . . . . . . . . . . . . . . . . 42

2.6.2 Calculation of average power and average velocity . . . . . . . . 44

2.6.3 Discussion . . . . . . . . . . . . . . . . . . 45

2.7 Conclusion . . . . . . . . . . . . . . . . . . . . . . . 47

3 Analysis of Operating Point Space 49

3.1 Constraints and limits . . . . . . . . . . . . . . . . . . . . . 49

3.1.1 Take-off constraint . . . . . . . . . . . . . . . . 49

3.1.2 Sliding constraint . . . . . . . . . . . . . . . 51

3.1.3 Fall-back constraint . . . . . . . . . . . . 52

3.1.4 Steady-state constraint . . . . . . . . . . . . . 52

$3.1 .5 v_{0} \approx v_{m}$ constraint .................... 53

3.2 Optimal walker . . . . . . . . . . . . . . . . . 54

3.2.1 Computation of average power and average velocity . . . . . . 54

3.2.2 Minimum mechanical power for inverted pendulum walker . . . . 55

3.3 Results . . . . . . . . . . . . . . . . . . . 57

3.3.1 Regions of operation . . . . . . . . . . . . 57

3.3.2 Optimal loci . . . . . . . . . . . . . . . . . 58 
3.3 .3 Discussion . . . . . . . . . . . . . . . . . 60

3.4 Conclusion . . . . . . . . . . . . . . . . . . . . 60

4 Hardware Design and Control Topology 63

4.1 Hardware design . . . . . . . . . . . . . . . . . 63

4.1.1 Mechanical subsystem ................. 64

Rimless wheels . . . . . . . . . . . . . . . . 64

Frame ......................... 66

Enclosures . . . . . . . . . . . . . . . . 68

4.1 .2 Electrical subsystem . . . . . . . . . . . . . . . . . 68

Energy source . . . . . . . . . . . . . . . . 69

Actuators . . . . . . . . . . . . . . . 69

Electronics . . . . . . . . . . . . . . . . . 70

CIPOS $^{\text {TM }}$ inverter board: . . . . . . . . . . . . . . . 71

Chatur controller board: . . . . . . . . . . . . . 72

4.1 .3 System integration . . . . . . . . . . . . . . 77

4.1.4 Load and actuator torque profile . . . . . . . . . . . 77

4.2 Control topology . . . . . . . . . . . . . . . . . . . . 78

4.2 .1 Torque control . . . . . . . . . . . . . . . . . . . . . 79

4.2.2 Current feedback . . . . . . . . . . . . . . . . 80

4.2 .3 Torque reference generation . . . . . . . . . . . . . . 82

4.2.4 Wheel synchronization . . . . . . . . . . . . . . 83

4.3 Experimental results . . . . . . . . . . . . . . . . . . . . . . 87

4.4 Conclusion . . . . . . . . . . . . . . . . . . . . . . . 88

5 Conclusion $\quad 89$

5.1 Summary of contributions . . . . . . . . . . . . . . 89

5.2 Scope for further work . . . . . . . . . . . . . . . . . . . . . . 91

A Jansen Mechanism $\quad 93$

A.1 Forward kinematics . . . . . . . . . . . . . . . . . . . . . . . . . 93

A.2 Inverse dynamics $\ldots \ldots \ldots \ldots$. . . . . . . . . . . . . . . . . . 97

B CAD Details $\quad 103$ 
B.1 Mechanical subsystem: AutoCAD drawings . . . . . . . . . . . . 103

B.2 Electrical subsystem . . . . . . . . . . . . . . . . . . . . 112

$\begin{array}{ll}\text { C List of Publications } & 141\end{array}$

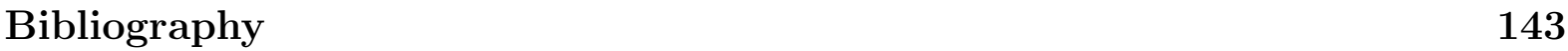




\section{List of Figures}

1.1 Energy budget in locomotion . . . . . . . . . . . . . . . . 3

1.2 Energy flow in locomotion . . . . . . . . . . . . . . 5

1.3 Gaits in legged locomotion . . . . . . . . . . . . . . . 6

1.4 Description of human walking . . . . . . . . . . . . . . 12

1.5 GRF patterns during stance phase in walking and running . . . . . . . 13

1.6 Models of walking . . . . . . . . . . . . . . . . . 14

1.7 Scope of the thesis . . . . . . . . . . . . . . . . . 18

2.1 Free body diagram of rimless wheel based walker. . . . . . . . . . . 22

2.2 Forward motion of inverted pendulum walker. . . . . . . . . . . . . 23

2.3 Fall angle and CoM velocity of inverted pendulum walker. . . . . . . . . 24

2.4 Ground impact loss models . . . . . . . . . . . . . . . 26

2.5 Actuation torque in human walking . . . . . . . . . . . . . 29

2.6 Analytical and numerical solutions for inverted pendulum dynamics . . . 31

2.7 Portion of stance phase for which actuation is present . . . . . . . . 33

2.8 Regime 1 and regime 2 walking . . . . . . . . . . . . . . . 36

2.9 Regime 3 and regime 4 walking . . . . . . . . . . . . . . . . . 37

2.10 Regime 5 walking . . . . . . . . . . . . . . . . . . . . . . . 38

2.11 Phase portraits for all five torque regimes . . . . . . . . . . . . . . 39

2.12 Experimental waveforms for regime 1 walking . . . . . . . . . . . . 42

2.13 Experimental waveforms for regime 2 walking . . . . . . . . . . . 43

2.14 Experimental waveforms for regime 3 walking . . . . . . . . . . . 43

2.15 Experimental waveforms for regime 4 walking . . . . . . . . . . . . 43

2.16 Average mechanical power versus average forward velocity . . . . . . . . 45

2.17 Mechanical CoT versus normalized average forward velocity . . . . . . . 45 
3.1 Take-off constraint lines. . . . . . . . . . . . . . . . . . 50

3.2 Sliding constraint lines for various values of coefficient of static friction . 51

3.3 Fall back constraint lines. . . . . . . . . . . . . . . . . . . . . . . 52

3.4 Steady-state constraint lines. . . . . . . . . . . . . . . . . . . . . 52

$3.5 v_{0} \approx v_{m}$ constraint lines .................... 53

3.6 Contour plots and construction of optimal locus . . . . . . . . . . . 56

3.7 Regions of operation . . . . . . . . . . . . . . . 57

3.8 Effect of changing $l$ on optimal locus and the corresponding $P_{\text {avg }}$ and CoT 59

4.1 Construction of a rimless wheel . . . . . . . . . . . . . . 65

4.2 Full mechanical assembly of Chatur . . . . . . . . . . . . . . . . 66

4.3 Block diagram of electrical system . . . . . . . . . . . . . . . . . . . . 68

4.4 Block rotor test of BLDC motor . . . . . . . . . . . . . . . . . . 70

4.5 Current directions of BLDC motor f . . . . . . . . . . . . . . . 70

4.6 Circuit schematic of inverter and BLDC motor . . . . . . . . . . . . . . 71

4.7 PCBs designed for Chatur . . . . . . . . . . . . . . . . . . 71

4.8 Electrical wiring diagram . . . . . . . . . . . . . . . . . . . . 75

4.9 Full system after integration . . . . . . . . . . . . . 76

4.10 Load and actuator torque profile for rimless wheel . . . . . . . . . . 78

4.11 Block diagram of torque control loop . . . . . . . . . . . . . . . . 79

4.12 Block diagram of the full control scheme . . . . . . . . . . . . . . . . . . 79

4.13 Reconstructing $I_{f b}$ from BLDC motor phase currents . . . . . . . . . . . 81

4.14 Strategy for obtaining motor currents once in every PWM cycle . . . . . 82

4.15 Flowchart of full control scheme $(1 / 2) \ldots \ldots$. . . . . . . . . . . . . 85

4.16 Flowchart of full control scheme $(2 / 2) \ldots \ldots$. . . . . . . . . . 86

4.17 Waveforms validating the working of the control scheme . . . . . . . . 88

A.1 Jansen leg mechanism at various crank angles . . . . . . . . . . . . 95

A.2 Trajectories of various points in Jansen leg mechanism . . . . . . . . . . 96

A.3 Links undergoing pure rotation . . . . . . . . . . . . . . . . . . . . . 98

A.4 Bond graph model for links undergoing pure rotation . . . . . . . . . . . 98

A.5 Links undergoing rotation and translation . . . . . . . . . . . . 100

A.6 Bond graph model for links undergoing rotation and translation . . . . . 100 
A.7 Bond graph model of Jansen Mechanism . . . . . . . . . . . . . . . 101

A.8 GRF, motor torque and link angular positions for Jansen mechanism . . 102

B.1 CAD drawing of annular disc . . . . . . . . . . . . . . . . . . 104

B.2 CAD drawing of Chatur frame: axle (main frame) . . . . . . . . . . 105

B.3 CAD drawing of Chatur frame: battery carrier (top piece) and axle . . . 106

B.4 CAD drawing of Chatur frame: battery carrier (bottom piece) . . . . . . 107

B.5 CAD drawing of Chatur frame: battery carrier (full) and axle . . . . . 108

B.6 CAD drawing of Chatur frame: tail piece . . . . . . . . . . . . . . 109

B.7 CAD drawing of Chatur frame: all parts . . . . . . . . . . . . . . 110

B.8 CAD rendering of enclosure for electronics . . . . . . . . . . . . . . . 111

B.9 CAD rendering of enclosure for terminal blocks . . . . . . . . . . . 111

B.10 CIPOS ${ }^{\top \mathrm{T}}$ inverter board OrCAD schematics . . . . . . . . . . . . . . 113

B.11 Chatur controller board gEDA schematics: Page-1 . . . . . . . . . . . . . 114

B.12 Chatur controller board gEDA schematics: Page-2 . . . . . . . . . . . . . 115

B.13 Chatur controller board gEDA schematics: Page-3 . . . . . . . . . . 116

B.14 Chatur controller board gEDA schematics: Page-4 . . . . . . . . . . . . 117

B.15 Chatur controller board gEDA schematics: Page-5 . . . . . . . . . . 118

B.16 PSoC ${ }^{\circledR}$ top-level schematics: Page-1 . . . . . . . . . . . . . . . . 123

B.17 PSoC ${ }^{\circledR}$ top-level schematics: Page-2 . . . . . . . . . . . . . . . . . 124

B.18 PSoC ${ }^{\circledR}$ top-level schematics: Page-3 . . . . . . . . . . . . . . 125

B.19 PSoC ${ }^{\circledR}$ top-level schematics: Page-4 . . . . . . . . . . . . . . . . 126 



\section{List of Tables}

1.1 Coefficient of rolling resistance for some common pairs of surfaces . . . . 2

1.2 Comparison of the three types of walking . . . . . . . . . . . . 7

2.1 Parameter values used for the torque regime waveforms . . . . . . . . . . 34

2.2 Specifications of Chatur . . . . . . . . . . . . . . . . 41

2.3 Measurements from experimental waveforms . . . . . . . . . . . . . 46

4.1 Specifications of sealed lead acid batteries . . . . . . . . . . . . 69

4.2 Specifications of BLDC motor . . . . . . . . . . . . . . 70

4.3 Commutation sequence (CCW rotation) of BLDC motor . . . . . . . . 70

4.4 CIPOS $^{T M}$ inverter board specifications . . . . . . . . . . . . . . 72

4.5 Chatur controller board specifications . . . . . . . . . . . . . 73

4.6 Specifications of flyback power supply . . . . . . . . . . . . . . . . 74

4.7 Time scales of various events/processes . . . . . . . . . . . . . . . . 83

A.1 Link lengths in Jansen leg mechanism in proportion to crank length. . . . 94

B.1 $\mathrm{PSoC}^{\circledR}$ port map . . . . . . . . . . . . . . . . . . . . . . 120 



\section{List of Symbols}

\begin{tabular}{|c|c|c|}
\hline Symbol & Units & Description \\
\hline$\beta$ & - & Duty factor \\
\hline$\gamma$ & $\mathrm{rad}$ & Slope angle of downward incline (for passive walker) \\
\hline$\lambda$ & $\mathrm{m}$ & Stride length \\
\hline$\mu_{r}$ & - & Coefficient of rolling resistance \\
\hline$\mu_{s}$ & - & Coefficient of static friction between foot point and grounc \\
\hline$\omega_{n}$ & $\mathrm{rad} / \mathrm{s}$ & Quantity $\left(\sqrt{\frac{g}{l}}\right)$ used for normalizing angular velocities \\
\hline$\Omega$ & $\mathrm{rad} / \mathrm{s}$ & CoM angular velocity \\
\hline$\phi$ & $\mathrm{rad}$ & Instantaneous angle of fall of the inverted pendulum \\
\hline$\Delta \phi$ & $\mathrm{rad}$ & Angle swept by inverted pendulum while being actuated \\
\hline$\phi_{a}$ & $\mathrm{rad}$ & Actuation torque parameter \\
\hline$\phi_{b}$ & $\mathrm{rad}$ & Braking torque parameter \\
\hline$\phi_{c}$ & $\mathrm{rad}$ & Difference between actuation \& braking torque parameters \\
\hline$\phi_{f}$ & $\mathrm{rad}$ & Final condition (for a given sub-phase) of fall angle \\
\hline$\phi_{m}$ & $\mathrm{rad}$ & Step angle or stance angle \\
\hline$\phi_{i}$ & $\mathrm{rad}$ & Initial condition (for a given sub-phase) of fall angle \\
\hline$\tau_{a}$ & $\mathrm{Nm}$ & Actuation torque \\
\hline$\tau_{b}$ & $\mathrm{Nm}$ & Braking torque \\
\hline$a_{z}$ & $\mathrm{~m} / \mathrm{s}^{2}$ & Accelerometer output, acceleration along z-axis \\
\hline$d$ & $\mathrm{~m}$ & Distance of travel; Duty ratio of power switches \\
\hline$d_{i}$ & - & Temporal duty ratio of actuator torque (current) \\
\hline$d_{i s}$ & - & Spatial duty ratio $\left(\Delta \phi / 2 \phi_{m}\right)$ of actuator torque (current) \\
\hline$E_{a}, E_{b}, E_{c}$ & $\mathrm{~V}$ & Motor winding back EMF \\
\hline$E_{b r k}$ & $\mathrm{~J}$ & Energy loss in braking \\
\hline$E_{\text {cont }}$ & $\mathrm{J}$ & Energy supplied continuosly for locomotion \\
\hline$E_{\text {ext }}$ & $\mathrm{J}$ & External energy, to move the CoM w.r.t. surroundings \\
\hline$E_{i m p}$ & $\mathrm{~J}$ & Energy loss in ground impacts (at heel strike) \\
\hline
\end{tabular}




\begin{tabular}{|c|c|c|}
\hline Symbol & Units & Description \\
\hline$E_{\text {init }}$ & $\mathrm{J}$ & Initial energy, to raise and accelerate the CoM of the walker \\
\hline$E_{\text {int }}$ & $\mathrm{J}$ & Internal energy, to move parts of locomotor w.r.t. its CoM \\
\hline$E_{i n v}$ & $\mathrm{~J}$ & $\begin{array}{l}\text { Energy invested in building and maintaining the locomotor } \\
\text { and any support infrastructure (road, rail etc.) }\end{array}$ \\
\hline$E_{k}$ & $\mathrm{~J}$ & Kinetic energy \\
\hline$E_{\text {loss }}$ & $\mathrm{J}$ & Energy losses \\
\hline$E_{p}$ & $\mathrm{~J}$ & Gravitational potential energy \\
\hline$E_{\text {phase }}$ & $\mathrm{J}$ & Mechanical energy supplied to the walker in a stance phase \\
\hline$E_{\text {total }}$ & $\mathrm{J}$ & Total energy for locomotion \\
\hline$f$ & $\mathrm{~Hz}$ & Step frequency \\
\hline$F_{c}$ & $\mathrm{~N}$ & Compression in the stance leg (fully extended) \\
\hline$F_{g r}$ & $\mathrm{~N}$ & Ground reaction force \\
\hline$F_{y}$ & $\mathrm{~N}$ & Vertical ground reaction force \\
\hline$g$ & $\mathrm{~m} / \mathrm{s}^{2}$ & Acceleration due to gravity $\left(9.81 \mathrm{~m} / \mathrm{s}^{2}\right)$ \\
\hline$\Delta h$ & $\mathrm{~m}$ & CoM height variation \\
\hline$I_{\max }$ & $\mathrm{A}$ & Rated motor current \\
\hline$I_{a}, I_{b}, I_{c}$ & $\mathrm{~A}$ & Motor phase currents \\
\hline$I_{f b}$ & A & Motor torque feedback \\
\hline$I_{\text {ref }}$ & A & Motor torque reference \\
\hline$K_{i}$ & - & Integral controller gain \\
\hline$K_{p}$ & - & Proportional controller gain \\
\hline$K_{\text {sync }}$ & - & Scaling factor for wheel synchronization \\
\hline$K_{t}$ & $\mathrm{Nm} / \mathrm{A}$ & Motor torque constant \\
\hline$l$ & $\mathrm{~m}$ & Leg length \\
\hline$l_{s}$ & $\mathrm{~m}$ & Step length \\
\hline$l_{s w}$ & $\mathrm{~m}$ & Effective length of swing leg \\
\hline$L_{a}, L_{b}, L_{c}$ & $\mathrm{H}$ & Motor winding inductance \\
\hline$m$ & $\mathrm{~kg}$ & Locomotor mass \\
\hline$m_{l e g}$ & $\mathrm{~kg}$ & Leg mass, considered for internal energy computation only \\
\hline$P_{a v g}$ & $\mathrm{~W}$ & Average mechanical power consumption \\
\hline$P_{\text {cont }}$ & W & Power delivered continuously to the locomotor \\
\hline$q$ & - & Shape factor \\
\hline$r_{g y r}$ & - & $\begin{array}{l}\text { Radius of gyration of rimless spoked wheel normalized by } \\
\text { spoke (leg) length }\end{array}$ \\
\hline
\end{tabular}


xxiii

$\begin{array}{lll}\text { Symbol } & \text { Units } & \text { Description } \\ R_{a}, R_{b}, R_{c} & \Omega & \text { Motor winding resistance } \\ t & \mathrm{~s} & \text { Time } \\ T & \mathrm{~s} & \text { Step duration or stance phase duration } \\ T_{s p} & \mathrm{~s} & \text { Sub-phase duration } \\ v & \mathrm{~m} / \mathrm{s} & \text { CoM velocity } \\ v_{0} & \mathrm{~m} / \mathrm{s} & \text { Initial mid-stance CoM velocity } \\ v_{0 s s} & \mathrm{~m} / \mathrm{s} & \text { Mid-stance CoM velocity at steady state } \\ v_{i} & \mathrm{~m} / \mathrm{s} & \text { Initial condition (for a given sub-phase) of CoM velocity } \\ v_{m} & \mathrm{~m} / \mathrm{s} & \text { Pre-impact CoM velocity } \\ v_{m}^{\prime} & \mathrm{m} / \mathrm{s} & \text { Post-impact CoM velocity } \\ v_{n} & \mathrm{~m} / \mathrm{s} & \text { Quantity }(\sqrt{g l}) \text { used for normalizing velocities } \\ v_{x, a v g} & \mathrm{~m} / \mathrm{s} & \text { Average forward velocity } \\ v_{x} & \mathrm{~m} / \mathrm{s} & \text { Horizontal component of CoM velocity } \\ V_{d c} & \mathrm{~V} & \text { DC link voltage of inverter } \\ W & \mathrm{~N} & \text { Weight of the locomotor }\end{array}$





\section{List of Abbreviations}

\section{Acronym Description}

2D Two Dimensional

3D Three Dimensional

ADC Analog-to-Digital Converter

AF Actuated Fall (of CoM)

AR Actuated Rise (of CoM)

ASK Amplitude Shift Keying

BLDC Brushless Direct Current (motor)

CAD Computer Aided Design

CCW Counter Clockwise

chan channel select (of analog multiplexer at ADC input)

CIPOS $^{\text {TM }}$ COntrol Integrated POwer System (from Infineon)

CoM Center of Mass

CoT Cost of Transport

CW Clockwise

DAC Digital-to-Analog Converter

DMA Direct Memory Access

DoF Degree of Freedom

EMA Exponential Moving Average (digital filter)

EMF Electromotive Force

GRF Ground Reaction Force

IGBT Insulated Gate Bipolar Transistor

LED Light Emitting Diode

LIPM Linear Inverted Pendulum Model

MEMS Micro-Electro-Mechanical System

MOSFET Metal Oxide Semiconductor Field Effect Transistor

MS Mild Steel 


\section{Acronym Description}

$\begin{array}{ll}\text { Mux } & \text { Multiplexer } \\ \text { PCB } & \text { Printed Circuit Board } \\ \text { PI } & \text { Proportional-Integral (controller) } \\ \text { PSoC }{ }^{\circledR} & \text { Programmable System on Chip (from Cypress Semiconductor) } \\ \text { PWM } & \text { Pulse Width Modulation } \\ \text { R1 } & \text { Regime 1 } \\ \text { R2 } & \text { Regime 2 } \\ \text { R3 } & \text { Regime } 3 \\ \text { R4 } & \text { Regime } 4 \\ \text { R5 } & \text { Regime } 5 \\ \text { RF } & \text { Radio frequency } \\ \text { RPM } & \text { Revolutions Per Minute } \\ \text { RX } & \text { Receiver } \\ \text { SAR } & \text { Successive Approximation Register (type of ADC) } \\ \text { SLIP } & \text { Spring Loaded Inverted Pendulum } \\ \text { soc } & \text { start-of-conversion (of ADC) } \\ \text { SWG } & \text { Standard Wire Gauge } \\ \text { TX } & \text { Transmitter } \\ \text { UART } & \text { Universal Asynchronous Receiver Transmitter } \\ \text { UF } & \text { Unactuated fall (of CoM) } \\ \text { UR } & \text { Unactuated rise (of CoM) } \\ \text { wheg } & \text { wheel }+ \text { leg } \\ \text { ZMP } & \text { Zero Moment Point } \\ & \end{array}$




\section{Chapter 1}

\section{Introduction}

\subsection{Terrestrial locomotion alternatives}

Animals, man-made vehicles and mobile robots move about in their environment land, water and air - in myriad different ways. In the animal world, motion on land i.e. terrestrial locomotion is largely achieved using appendages such as legs driven by muscles acting as motors. Limbs facilitate great versatility of activities in animals. For instance, with the same set of limbs humans can hold, lift, catch, manipulate, crawl, walk, jog, run, hop, skip, jump, climb, swim, brachiate and what not. Among all these capabilities, picking out just those relating to locomotion still leaves a wide spectrum of capabilities that enables traversal of different kinds of terrains. Legged robots are only beginning to mimic a few of the versatile locomotion capabilities of land animals.

In the man-made world, an overwhelming majority of terrestrial vehicles and many mobile robots move using wheels on prepared surfaces because of its low energetic cost of transport. Wheeled systems are energy efficient under three assumptions.

1. The aerodynamic drag of the system is either negligible or minimized by design.

2. The wheel is rigid.

3. The surface of travel is hard and even (such as roads and tracks).

Assumptions (2) and (3) ensure minimal deformation of the wheel or the surface of travel and hence a low rolling resistance. Table 1.1 shows the orders of magnitude of the coefficient of rolling resistance for a few common pairs of surfaces. Note that rolling 
Table 1.1: Coefficient of rolling resistance for some common pairs of surfaces (Clark and Dodge, 1979; Lawyer, 2004; Coutermarsh, 2007)

\begin{tabular}{lc}
\hline Pair of surfaces & $\begin{array}{c}\text { Coefficient of rolling resistance } \\
\mu_{r}=(\text { drag force }) /(\text { normal reaction })\end{array}$ \\
\hline Steel wheels on steel rails & $\sim 0.001$ \\
Car tyre on tar/asphalt road & $\sim 0.01$ \\
Car tyre on loose sand & $\sim 0.1$ \\
\hline
\end{tabular}

resistance of car tyres on tar road is an order of magnitude higher than that for steel wheels on steel rails. Car tyre on loose sand is another order of magnitude worse. If tyre pressure is low or the surface of travel is soft and/or uneven leading to a poor coefficient of rolling resistance, use of wheels could be an energetically costly solution and, in some cases, not even a solution (tyres can skid in place; obstacles can be higher than wheel radius). In such cases, it might be better to resort to legged locomotion even in manmade systems. But legged systems are harder to construct and control. So they tend to be more expensive than wheeled systems.

\subsection{Energetics of locomotion}

For any means of locomotion, whatever is in motion can be referred to as the locomotor - animal, vehicle, robot. The complete energy budget of any mode of locomotion is shown in Figure 1.1 and is formulated by

$$
E_{\text {total }}=E_{i n v}+E_{\text {ext }}+E_{\text {int }}
$$

where $E_{i n v}$ is invested energy, $E_{\text {ext }}$ is external energy and $E_{i n t}$ is internal energy.

$E_{i n v}$ is energy invested in building and maintaining the locomotor and any other support infrastructure that it needs (holds for man-made locomotors only). Most of this energy is put in at the beginning of the life time of the system with periodic maintenance top-ups once in few months or years. Unlike wheeled vehicles, legged vehicles/robots do not need prepared surfaces such as roads or tracks. Hence, the invested energy in legged systems is lower compared to wheeled systems.

Consider locomotion on level ground where the center of mass (CoM) of the locomotor does not have to be continuously raised (or lowered). External energy $\left(E_{\text {ext }}\right)$ is the energy 


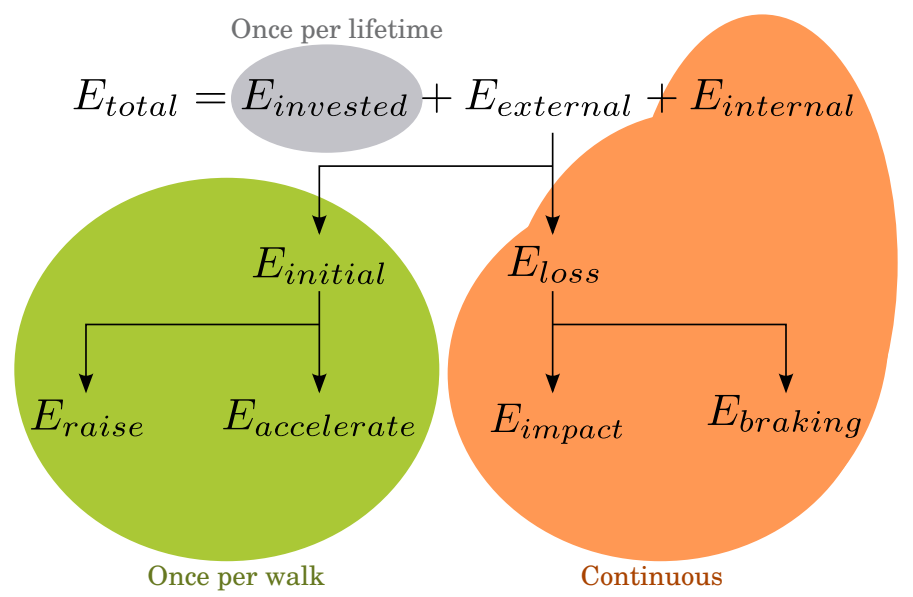

Figure 1.1: Energy budget in locomotion.

required to move the CoM with respect to its surroundings. It comprises two parts initial energy $\left(E_{\text {init }}\right)$ and energy losses $\left(E_{\text {loss }}\right)$.

$$
E_{\text {ext }}=E_{\text {init }}+E_{\text {loss }}
$$

For a legged system, at the start of a walk/run, some initial energy needs to be expended to raise and accelerate the CoM. This energy is put in only once per steady-state walk/run. A wheeled vehicle has a fixed CoM height above the surface of travel. The initial energy serves only to accelerate the vehicle to the desired speed. This is required once per stretch of constant speed travel. $E_{\text {loss }}$ is the energy expended to overcome losses in the system. Braking, ground impacts and aerodynamic drag are the loss mechanisms in a legged system. For a wheeled vehicle, losses are due to rolling resistance and aerodynamic drag.

Internal energy $\left(E_{\text {int }}\right)$ is the energy required to move the parts of a locomotor with respect to its CoM. Since the legs or wheels of a locomotor are much lighter than the full locomotor, the effect of $E_{i n t}$ is appreciable only at higher speeds. $E_{\text {loss }}$ and $E_{\text {int }}$ components need to be supplied continuously $\left(E_{\text {cont }}\right)$ in order to maintain a steady average speed.

$$
E_{\text {cont }}=E_{\text {loss }}+E_{\text {int }}
$$

The widely used dimensionless metric for comparing the energy efficiency of various modes of transport is specific resistance. It is also referred to as cost of transport (CoT) and is defined as

$$
\mathrm{CoT}=\frac{E_{\text {cont }}}{W d}=\frac{P_{\text {cont }}}{W v}
$$

where $W$ is the weight of the locomotor, $d$ is the distance of travel and $P_{\text {cont }}$ is the power that needs to be continuously delivered to the locomotor to achieve motion at a 
constant velocity $v$. Note that CoT is not a fixed value for any mode of transport. It increases with speed. Gabrielli and von Karman (1950) collated and plotted data on the specific resistance of various vehicles operating at a range of speeds. They used gross vehicle weight in their calculations. What needs to be transported is only the payload. Radtke (2008) uses the payload weight in his somewhat modified metric which he called thermal energetic economy. Tucker $(1970,1975)$ analyzed the energetic cost of transport for animals.

Figure 1.2 illustrates the energy flow in locomotion. The mechanical load is the body of the locomotor which has some inherent dynamics and loss mechanisms. It is driven by an actuator whose efficiency is high only in a given range of speeds and tapers off at higher and lower speeds.

The structure and function of animal bodies are solutions to optimization problems that involve multiple competing objectives - finding food and mates, escaping predation, defending territory and offsprings, and surviving harsh conditions. The muscles have a certain design that helps meet many of these challenges. Given this particular kind of actuator (muscle), animals try to choose gaits that minimize metabolic energy spent for any given speed (Minetti and Alexander, 1997). Srinivasan (2011) details the structure of gaits that minimize metabolic energy for a number of simple biped models. Any such optimization taking metabolic energy as the cost function involves an appropriate energetic model of the muscle. Note that a choice of gait does not necessarily guarantee minimum mechanical energy for the given speed.

As opposed to an animal, an artificial system can be optimized for any one criterion (if so desired), say energy. Hence, an engineer can construct and operate locomotors choosing gaits that minimize mechanical energy. Subsequently, based on the range of speeds of the mechanical load, an actuator can be designed that is most efficient in that range. This in turn will lead to the minimum energy input to the actuator. This approach is also useful while designing exo-skeletons for humans. 


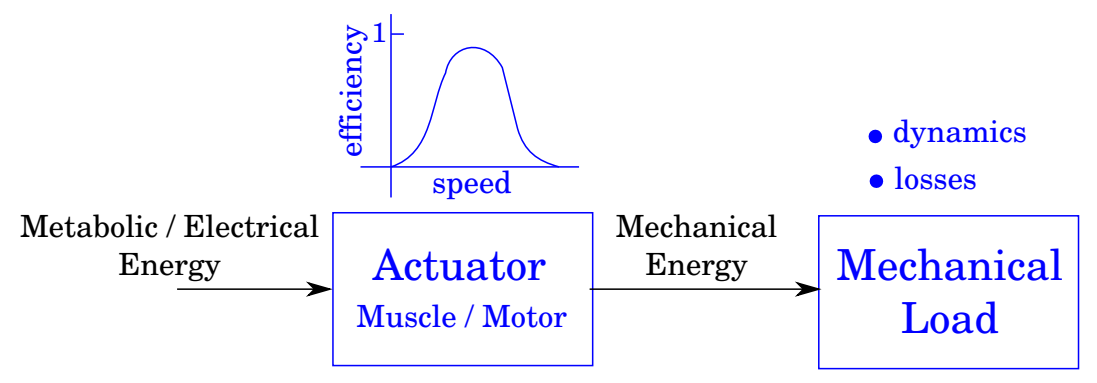

Figure 1.2: Energy flow in locomotion.

\subsection{Gaits in legged locomotion}

\subsubsection{Definition of gait}

Legged locomotion, whether in natural or artificial systems, can take on various possible forms or foot-fall patterns referred to as gaits. Humans walk at low speed and run at higher speed. Horses walk at low speed, trot at intermediate speed and gallop at higher speed. Alexander (1989) defined gait as "a pattern of locomotion characteristic of a limited range of speeds described by quantities of which one or more change discontinuously at transition to other gaits."

In the legged locomotion context, these quantities are duty factor (fraction of the stride duration for which a given foot is on the ground) and shape factor (ratio of Fourier coefficients describing the vertical ground reaction force acting on the foot; see Section 1.3.3). Alexander showed that for adult humans, the duty factor in walking is above 0.5 , typically in the range $0.55-0.70$. For running it is below 0.5 , typically in the range $0.3-$ 0.4. The changeover happens abruptly at around $2 \mathrm{~m} / \mathrm{s}$. Similarly, the shape factor also abruptly changes from positive values $(0.2-0.8)$ in walking to negative values $(-0.2-0)$ in running.

\subsubsection{Classification of gaits}

Various types of gaits (Figure 1.3) have been identified and studied in nature and subsequently mimicked in artificial systems. Some gaits are seen only in animals and some only in artificial systems. Gaits are classified into two broad categories - ballistic gaits and walking gaits.

Ballistic or running gaits have an air-borne phase when none of the legs are touching 


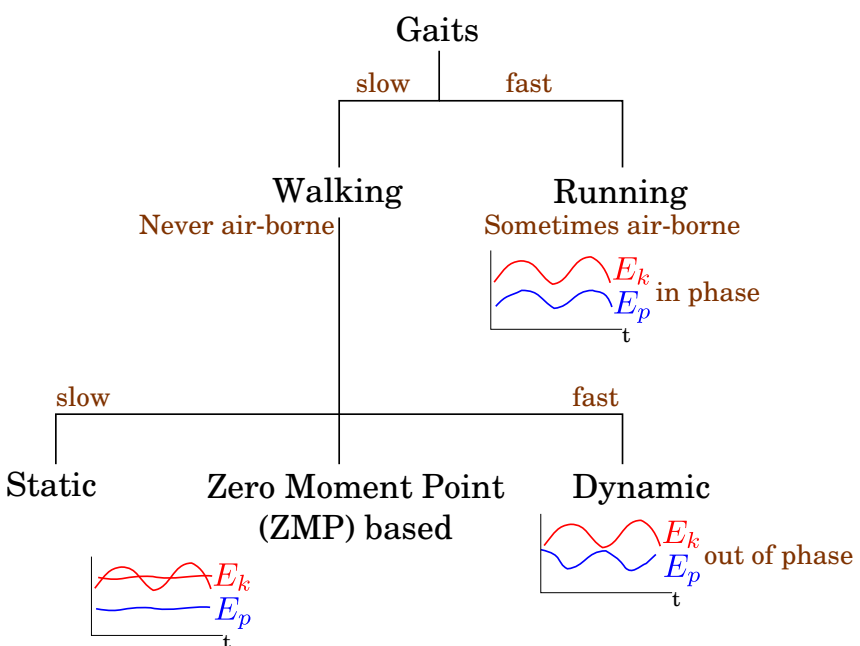

Figure 1.3: Gaits in legged locomotion. $E_{k}=$ Kinetic energy, $E_{p}=$ Potential energy.

the ground. In such gaits, the CoM trajectory resembles that of a bouncing ball and elastic strain energy plays a significant role. Kinetic energy $\left(E_{k}\right)$ and gravitational potential energy $\left(E_{p}\right)$ rise and fall in phase. Such gaits are used for travelling at high speed. In bipeds, ballistic gaits include jog, run, hop (on one leg) and jump (on both legs). Humans, birds and kangaroos exhibit biped ballistic gaits. Raibert built planar as well as 3D hopping and running robots having one, two and four legs (Raibert, 1986a,b). In quadrupeds, ballistic gaits include trot, pace, bound, gallop, canter and pronk. Several mammals - horses, deer, cats, dogs etc. - exhibit many of these gaits. Boston Dynamics built quadruped runners such as WildCat and Cheetah capable of galloping and bounding. Ballistic gaits are not addressed in this thesis.

In walking gaits, at least one leg is on the ground at all times. At no point of time is the walker completely air-borne. Generally, little energy gets stored as elastic strain energy. Such gaits are used for moving at low speed. They are further classified into static, Zero Moment Point (ZMP) based and dynamic walking. In static as well as ZMP-based walking, the CoM is largely maintained at a constant height leading to little variation in $E_{p} . E_{k}$ may or may not vary depending on the way the actuators are energized. In dynamic walking, $E_{p}$ and $E_{k}$ vary out of phase with one another. A brief discussion and literature review on each of these types of walking is given below. Table 1.2 compares the three types of walking. 
Table 1.2: Comparison of the three types of walking.

\begin{tabular}{llll}
\hline & Static & ZMP-based & Dynamic \\
\hline Stability & $\begin{array}{l}\text { High } \\
\text { (static stability) }\end{array}$ & $\begin{array}{l}\text { Low } \\
\text { (static stability) }\end{array}$ & $\begin{array}{l}\text { Intermediate } \\
\text { (orbital/cyclic stability) }\end{array}$ \\
\hline CoM position & Low & Intermediate & High \\
\hline Posture & Sprawling & Upright & Upright \\
(bent knees) & (extended knees) \\
\hline No. of legs & 4 or more & 2 & 2 and 4 \\
\hline required & High & High & Low \\
\hline Terrain & Uneven & Even & Intermediate \\
\hline Foot type & Point & Flat & Any \\
\hline Appearance & Natural & Unnatural & Natural \\
\hline Control & Conservative & Conservative & Optimistic \\
approach & (hardly any & natural dynamics) & natural dynamics) \\
(controller utilizes & natural dynamics) \\
\hline Speed & Slow & Slow & Fast \\
\hline
\end{tabular}

\section{Static walking}

In static walking, the system is always statically stable i.e. projection of the system's CoM on the ground always lies within the support polygon formed by the feet that are resting on the ground. At any instant, the walker is like a table or a tripod. It does not have a tendency to topple. Such walkers have four or more legs typically coming out at the sides of their torso/chassis. They tend to have a sprawling posture with a low CoM. Motion is slower than that in case of ZMP-based and dynamic walking.

Creeping is an example of static walking. It involves lifting and placing one leg at a time. A periodic creeping gait is called crawling. A non-periodic creeping gait is called a free gait. It is used in rough terrain to search for safe foot-holds, avoiding obstacles and maintaining static stability (Kajita and Espiau, 2008). When you are navigating over rocky terrain on all four limbs, you are using a free gait. Several animals are known 
to use variations of the creeping gait. Man-made walkers using creeping gaits include the GE quadruped walking truck (Mosher, 1969), the TITAN series of quadruped robots from Tokyo Institute of Technology (Hirose et al., 1997; Arikawa and Hirose, 1996; Kato and Hirose, 2001) and the LittleDog by Boston Dynamics (Murphy et al., 2010).

Many multipeds exhibit a kind of static walking where groups of legs are lifted and placed in synchronism. The tripod gait of hexapods involves lifting and placing three legs at a time in alternating triangles. Many insects like ants, termites, beetles and cockroaches use the tripod gait. Millipedes and centipedes exhibit metachronal wave gait where they lift and place several pairs of legs in unison. Static walking calls for usage of many legs and is mostly seen in smaller animals. There is no land animal weighing more than 100 g capable of walking on six legs (Gonzalez de Santos et al., 2006).

The Adaptive Suspension Vehicle - the largest hexapod in the world - built by Waldron and McGhee (1986) was capable of using free gait as well as tripod gait depending on the terrain. The COMET series from Chiba University (Nonami, 2002; Irawan et al., 2011; Irawan and Nonami, 2011) and IRobot's ARIEL are recent examples of manmade hexapods capable of tripod gait. Theo Jansen built several multi-legged walkers called Strandbeests. They exhibit static walking powered only by the beach wind (Jansen, 2014). The leg mechanism designed by Jansen is a planar linkage mechanism that mimics the leg motion pattern of a walking mammal.

Appendix A presents an analysis of the kinematics and dynamics of Jansen's one degree of freedom (1-DoF) leg mechanism. The kinematic analysis helps determine the foot point trajectory. The variation in step length and step height with change in crank radius is presented. The dynamic model is constructed using bond graph approach (Karnopp et al., 1990). This model helps determine the motor torque profile (and hence the torque rating) and the link as well as joint stresses for a given ground reaction force pattern.

About half of the earth's surface is presently inaccessible to wheeled vehicles (Knight and Nehmzow, 2002). The chief advantage of static walking multi-legged robots is their ability to negotiate such arduous terrain. Their applications span transporting of goods, civil construction, rescue operations and humanitarian demining among others.

\section{ZMP-based walking}

Static walking facilitates stable but slow motion in multipeds. For bipeds the legs come out under the torso/chassis and the support polygon is too small to make static stability 
practically possible. Vukobratovic and Juricic (1969) pioneered the zero moment point $(\mathrm{ZMP})^{*}$ principle which allows the projection of the CoM to leave the support polygon but ensures that the finite-sized stance foot always maintains flat contact with the ground.

Ichiro Kato and his successors from Waseda University, Japan designed and implemented several generations of bipeds using the ZMP principle - WL-series, WABOTseries, WABIAN-series (Lim and Takanishi, 2007). The Honda P-series culminating in the ASIMO (Sakagami et al., 2002; Honda, 2007) also used the ZMP approach. Other notable examples are Kawada's HRP series (Hirukawa et al., 2004) and Sony's QRIO (Nagasaka et al., 2004). The permeance of the ZMP concept in the robotics community over thirty-five years has been succinctly reviewed by Vukobratovic and Borovac (2004). There does not seem to be any examples of ZMP-based walking in animals except when human dancers mimic ZMP-based robots.

The control approach using the ZMP-criterion is conservative in that the inherent mechanical dynamics are damped out. Also, these walkers tend to keep their knees bent so as to lower their CoM thus improving stability. Both these factors - damping natural dynamics and holding knees in bent position - require the system to be actuated all the time. Hence, these walkers consume more energy and have an unnatural looking gait compared to human walking.

\section{Dynamic walking}

In dynamic walking - also called limit cycle walking — the system is statically unstable but stable in the sense that it is able to sustain a nominally repeating gait pattern without falling down. Hobbelen and Wisse (2007) defined limit cycle walking as "a nominally periodic sequence of steps that is stable as a whole but not locally stable at every instant in time." Consider a two-dimensional (2D) walker whose walking motion is restricted to its sagittal plane. The state of a $2 \mathrm{D}$ dynamic walker can be specified in terms of a displacement quantity (angular displacement $\phi$ of weight-bearing leg w.r.t vertical) and a velocity quantity (CoM velocity $v$ ). A plot between these two quantities - called the state space trajectory or phase portrait - provides a graphic visualization of the dynamics of the walker. For limit cycle walking, this state space trajectory is cyclic and is said to have orbital stability or cyclic stability (Strogatz, 2001). In the absence of energy losses, this trajectory would represent a particular mechanical energy level of the

\footnotetext{
*The term ZMP is not used in the paper; It was coined later.
} 
system akin to the orbital energy levels of electrons in atoms.

Such systems tend to have an upright posture with a high CoM. Like ZMP-based walkers, the legs come out under the torso. But the knees (if any) are mostly in fully extended position when bearing weight. The control approach is more adventurous as it tries to use the inherent mechanical dynamics of the system - inertia of motion and tipping moment due to gravity - instead of damping it completely. The gait looks natural. It is energy efficient as motion is largely achieved because of the pendular energy exchange between gravitational potential energy and kinetic energy. At every step, the collision of the foot against the ground causes some energy loss. This lost energy has to be compensated for in order to have a sustained gait. Losses can be reduced if some of the impact energy is stored in compliant elements rather than being dissipated. Dynamic walking is faster than static walking as well as ZMP-based walking (for the same spent energy) but slower than most ballistic gaits. In nature, several mammals (including humans) and birds exhibit dynamic walking.

Among man-made creations, passive (unactuated) dynamic walking was first seen in stiff-legged bipedal toys ${ }^{\dagger}$ that walked down shallow inclines. Various versions of these toys are described in several old patents (Fallis, 1888; Bechstein, 1912; Mahon, 1914; Wilson, 1938) and by McMahon (1984a,b). Inspired by these toys, McGeer (1990a,b) designed some impressive mechanisms that exhibit passive dynamic walking on gentle down slopes without and with knees. These 2D passive walkers need no control or energy inputs and are powered only by gravity. McGeer's 'bipeds' are four-legged with one inner pair of legs and one outer pair of legs. Each pair moves in unison resembling the walk of a man on crutches. Proceeding further on McGeer's ideas, Andy Ruina and his group developed several passive and passive-based walkers. Collins et al. (2001) built the first 3D passive dynamic walking robot with two legs and knees. The rolling and yawing tendencies in their prototype are kept in check using a special foot design and counter-swinging arms.

Adding actuators to passive walking systems make them capable of walking on level surfaces without the need for a ramp. Such dynamic walking systems with minimal actuation and control are also called under-actuated walkers. They use actuation at one or more of the three joints - ankle, knee and hip. Some salient examples are MIT's Spring Flamingo (Pratt and Pratt, 1999; Pratt, 2000), Cornell and Delft bipeds (Collins et al., 2005; Collins and Ruina, 2005), Delft's MIKE (Wisse and van Frankenhuyzen, 2006), Twente's Dribel (Dertien, 2005, 2006) and Cornell Ranger (Bhounsule et al., 2014).

\footnotetext{
${ }^{\dagger}$ sometimes called Wilson Walkies
} 


\section{Choosing a locomotion alternative}

If speed is of primal importance in a particular case of terrestrial locomotion, then hard wheels on hard and even surfaces is the best solution. The other option is to use legged systems employing ballistic gaits. But they can be more energy intensive than wheels unless the terrain is off road. Sometimes the ability to move with low energy expenditure could matter more than being able to move fast. For example, going on a jungle trail, in a desert or grassland, in planetary exploration and so on. Such off-road terrains, where people and animals can walk, are places where vehicles/robots could resort to dynamic walking gaits to get maximum energy efficiency albeit at low speed. The scope of this thesis lies in such gaits. On the other extreme, if the terrain is highly uneven, neither wheeled locomotion nor dynamic walking may be possible and static walking has to be resorted to, in the interest of stability.

\subsubsection{Description of gaits}

Visualization or graphical description of gaits can be done using foot marks, support sequences (Muybridge, 1957), gait diagrams (Hildebrand, 1965), photography and videography. Muybridge photographed gaits of several running mammals using a series of cameras triggered in rapid succession. His pioneering work played a key role in understanding animal gaits and in starting legged locomotion as a field of research.

Some of the terminology commonly used in legged locomotion literature to describe various aspects of a gait is now discussed. Take the case of a biped dynamic walker like a human. Figure 1.4 illustrates some of the terms associated with human walking. Each leg has a stance phase (when it is on the ground) and a swing phase (when it is in the air). At the start of a stance phase, there is an energy dissipating heel strike. Then the walker passively vaults over the stance leg like an inverted pendulum keeping the knee extended, the leg stiff and the foot flat on the ground. During this time, the swing leg is in retracted position. This helps prevent the swing leg from scuffing against the ground thus disturbing the inverted pendulum. The stance leg is vertical at mid-stance. Towards the end of a stance phase, toe-off happens where the calf muscles extend the ankle to push against the ground. This push-off is the 'power stroke' that replenishes the mechanical energy lost during heel strike. This ensures that the system has enough mechanical energy for the vaulting over the next stance leg to commence the way it happened in the previous stance phase. 


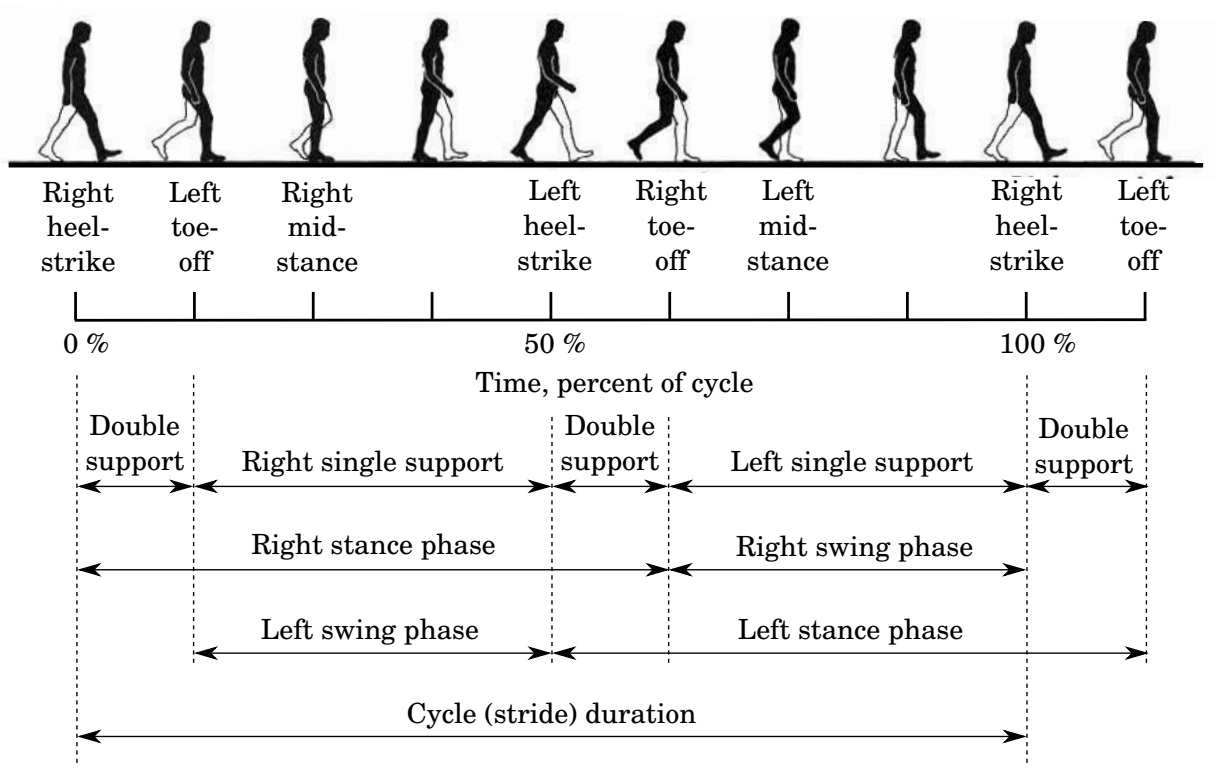

Figure 1.4: Description of human walking. Illustration adapted from Inman et al. (1981).

A complete walking cycle is called a stride. From the heel strike of a leg to the next heel strike of the same leg comprises a stride. Fraction of the stride duration for which a given foot is on the ground is called duty factor $(\beta)$. The two legs of a biped alternately switch roles as stance and swing legs with a small overlap period known as double support. The double support duration as a fraction of the stride duration can be expressed as $2 \beta-1$. So a biped dynamic walker with a duty factor of 0.6 has a double support duration that is $20 \%$ of the stride duration. The horizontal distance moved in one stride is called stride length $(\lambda)$. The reciprocal of the stride duration is called stride frequency. An event happening on one leg to the corresponding event on the other leg comprises a step. The distance moved in one step is called step length and the reciprocal of step duration is called step frequency or cadence. Note that step lengths for left and right legs need not be equal.

During a stance phase in walking and running, the characteristic ground reaction force (GRF) patterns experienced at the foot are shown in Figure 1.5 (Geyer, 2005). The vertical component of GRF tends to have a two-hump ${ }^{\ddagger}$ shape for dynamic walking and a bell shape for running. The double-hump shape is seen not just in normal human walking but also in tip-toe and high-heel walking as well as in walking ostriches (Usherwood et al., 2012). For a stance duration extending from $-\tau / 2$ to $+\tau / 2$, Alexander and Jayes (1980)

\footnotetext{
‡Like a Bactrian camel’s back
} 

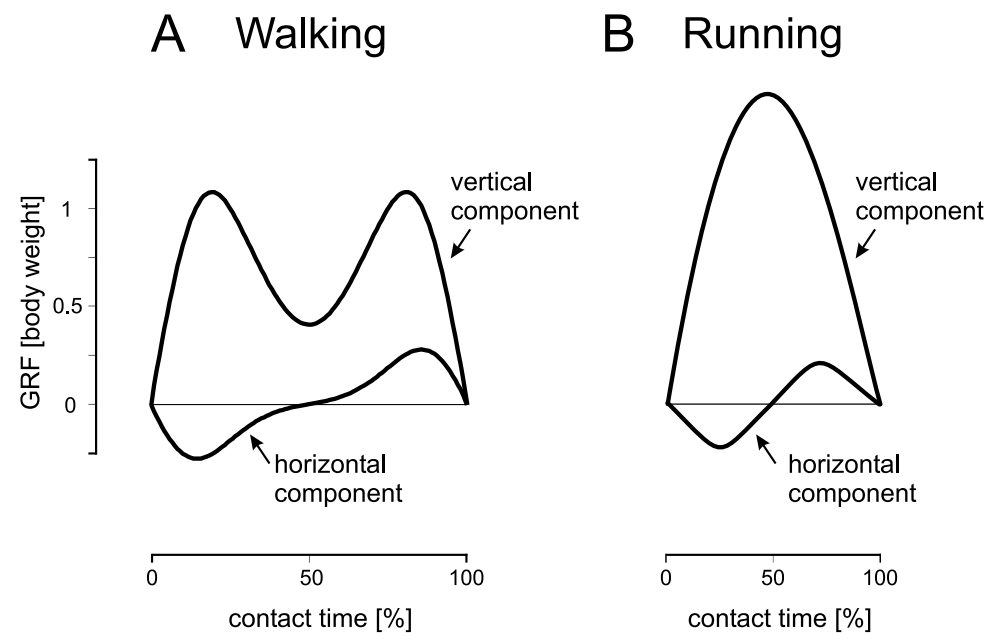

Figure 1.5: Characteristic ground reaction force (GRF) patterns observed during the stance phase in walking (A) and running (B). Illustration taken from Geyer (2005) with permission.

expressed the vertical GRF as follows:

$$
F_{y}=A\left[\cos \left(\frac{\pi t}{\tau}\right)-q \cos \left(\frac{3 \pi t}{\tau}\right)\right]
$$

where $\mathrm{A}$ is chosen so as to make the mean value of $F_{y}$, over a complete stride, equal to body weight. The parameter $q$ is called shape factor as it determines whether the force is two-humped (positive $q$ ) or bell-shaped (negative $q$ ) or anywhere in between. The horizontal component of GRF is negative in the first half of the stance phase and positive during the second half.

\subsection{Models of walking}

Several models for walking (Figure 1.6) have been proposed and analyzed. These models capture the essential aspects of human walking to varying degrees. They have been subsequently used for designing and building robotic walkers.

\subsubsection{Inverted pendulum model}

The simplest and probably the most elegant model for 2D dynamic walking is the inverted pendulum model (Figure 1.6a). Alexander (1976) describes it as the minimal bipedal walker. It comprises repeated vaulting of the CoM (mass $m$ ) over a rigid massless leg of fixed length $(l)$. The rigid leg assumption draws on the fact that humans typically 

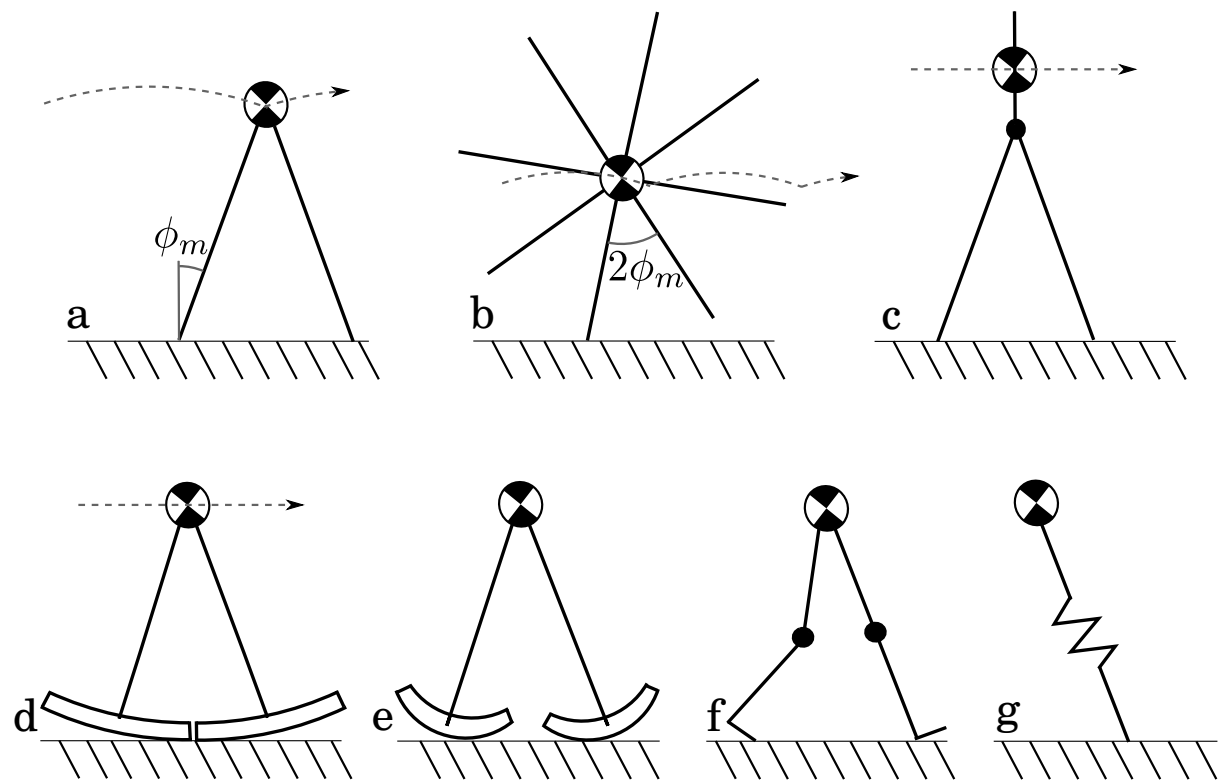

Figure 1.6: Models of walking: (a) Inverted pendulum, (b) Rimless spoked wheel, (c) Linear inverted pendulum (d) Synthetic wheel, (e, f) Inverted pendulum with heavy legs, without and with knees, (g) Spring loaded inverted pendulum. Adapted from Alexander (1995).

keep the knee of the weight-bearing leg fully extended. The massless leg assumption is permissible since the mass of the leg is much smaller than the total mass of the biped. The two legs alternate their roles of bearing the weight (stance) and being in the air (swing). The CoM rises and falls tracing a series of arcs of radius equal to the leg length.

Assuming symmetry of the CoM trajectory about mid-stance, the step angle $\left(\phi_{m}\right)$ is the angle made by the stance leg with the vertical at the beginning and the end of a stance phase. If the step lengths of both legs are equal, the step angle is half the inter-leg angle at heel strike. At any arbitrary instant during the stance phase the angle of fall of the inverted pendulum is denoted as $\phi$. The maximum fall angle equals the step angle. The angle made by the new stance leg with respect to the ground is called the angle of attack and equals $90^{\circ}-\phi_{m}$.

The step length $\left(l_{s}\right)$ and the CoM height variation $(\Delta h)$ predicted by the inverted pendulum model are as follows.

$$
\begin{gathered}
l_{s}=2 l \sin \phi_{m} \\
\Delta h=l\left(1-\cos \phi_{m}\right)
\end{gathered}
$$

The CoM height variation over a stance phase of a human is empirically found to be about $40 \mathrm{~mm}$ (Margaria, 1976). Assuming a leg length of $1 \mathrm{~m}$, this gives a step angle of about $16^{\circ}$. The corresponding step length works out to $0.55 \mathrm{~m}$. The step angle as well as 
the step length calculated from the model are lesser than observed typical values $-20^{\circ}$ and $0.7 \mathrm{~m}$. This can be explained by a flexible finite-sized foot as opposed to a point foot. Such a foot leads to a finite double support duration during which the walking deviates from the inverted pendulum model. The CoM trajectory near its minimum height point is smoothened out as shown by Cavagna et al. (1977). The inverted pendulum model with point feet implies instantaneous support transfer from one leg to the other. This only allows for an infinitesimally small double support duration.

Since gravity provides the centripetal acceleration for the CoM, the model predicts a maximum possible mid-stance speed $v_{0}$ of $\sqrt{g l}$ which evaluates to $3.1 \mathrm{~m} / \mathrm{s}$. Usherwood (2005) argues that an average forward speed of $\sqrt{g l}$ can only be realized for step angle close to zero and prohibitively high step frequencies that are not practically realizable. Using basic mechanics, he proves that the maximum realizable mid-stance speed (and hence average forward speed) is lower than $\sqrt{g l}$ for fall angles larger than zero. The upper limit for the step angle is about $48^{\circ}$ which can be realized only for close to zero mid-stance speed (see Figure 3.1). Note that this upper limit is independent of leg length and gravitational acceleration.

Race walkers are able to achieve up to $4.4 \mathrm{~m} / \mathrm{s}$ - much greater than $3.1 \mathrm{~m} / \mathrm{s}$ which implies that their walking gait is not akin to an inverted pendulum. This has been validated by two observations. (1) They move their hips in peculiar ways so that the arc formed by the CoM trajectory has a radius larger than leg length (Alexander, 1996). (2) Even though they are supposed to be walking, slow motion videos have shown that they indeed have an air-borne phase (Pavei et al., 2014).

Garcia et al. (1998) show numerically and analytically that the compass gait of the inverted pendulum model explains the passive dynamic walking achieved by McGeer's walkers down an incline, when the legs have finite point masses at the foot. Also, a substantial metabolic cost associated with step-to-step transitions in human walking is because of walking like an inverted pendulum (Kuo et al., 2005; Kuo, 2007). The inverted pendulum model thus explains several aspects of bipedal dynamic walking and is hence preferred in this work over other multi-body models.

\subsubsection{The rimless wheel}

The problem of ground scuffing in bipeds caused by the swing leg can be solved in many ways. Bipedal ramp-walking toys tackled it by rocking from side to side. Another solution 
is to have retractable legs using knees or telescopic legs. This would require an extra actuator (per leg) that actuates the retraction. The rimless spoked wheel (Figure 1.6b), actuated at the hub, facilitates a repeating sequence of inverted pendulum vaults without the added cost and complexity of extra motors for swing leg retraction/actuation.

Bekker (1961) mentioned the conceptual similarity in the CoM kinematics and dynamics of a walking biped and that of a rolling polygon. Much like a rolling polygon, a rimless wheel, with only one motor at its center, could serve as a model for the inverted pendulum walking of bipeds. It can be pictured as a wagon wheel with the rim removed and having sturdy spokes that can bear large compressive forces (Margaria, 1976). The rolling motion of the rimless spoked wheel can be described as a 'walk' since the locomotor's point of contact with the ground shifts discontinuously from one spoke (leg) to another.

Dynamics of a passive rimless wheel rolling down a slope have been analyzed for both 2D (McGeer, 1990a) and 3D (Coleman et al., 1997) cases. Smith and Berkemeier (1998) modelled the motion of a finite-width rimless wheel in 3D. The consecutive spokes in their model are on alternate sides of the wheel thus taking into account the finite leg separation in humans or robots. Passive walking rimless wheels (on downward incline) with springy feet and visco-elastic legs have been demonstrated (Narukawa et al., 2009; Asano et al., 2012). Rimless wheel actuated by radially expanding legs have also been explored (Asano, 2009; Yan and Agrawal, 2004). There does not seem to be any attempt at a hub actuated rimless wheel walker. OutRunner built by Robotics Unlimited uses the rimless wheel approach for running (Robotics-Unlimited, 2014). It uses springy legs that help store and release the impact energy.

Rimless wheels with various number and shapes of spokes have been employed as solutions for traversing difficult terrains. European Space Agency's PRELERO used six such 'wheels' each with only one spoke (Martin-Alvarez et al., 1996). Boston Dynamics' RHex also used six of them each having three arc-shaped spokes (Saranli et al., 2001). The Biorobotics Group at Case Western Reserve University built the WHEGS ${ }^{\mathrm{TM}}$ series of robots using three straight spokes per rimless wheel (Quinn et al., 2002; Morrey et al., 2003; Allen et al., 2003).

Existing prototypes of the rimless wheel focus either on its passive walking on a downward incline or on its multi-terrain capabilities. The present work explores their potential for energy efficiency when used as dynamic walkers in normal terrains (places where humans can walk normally). The rolling rimless wheel serves as a platform for 
investigating the dynamics and energetics of inverted pendulum walking with constant step angle.

\subsubsection{Other models}

Kajita et al. (1990) proposed the Linear Inverted Pendulum Model (LIPM; Figure 1.6c) for bipedal walking. It assumed a constant CoM height throughout the walk. They designed and implemented a 2D biped and later a 3D biped (Kajita et al., 2001) using the LIPM approach. Though the LIPM has enabled the implementation of several successful biped walkers, these walkers are quite energy intensive on account of being ZMP based. Luo et al. (2013) proposed the possibility of achieving limit cycle walking using LIPM. Even then, the LIPM does not capture the vertical excursions of the CoM observed in natural walkers.

McGeer (1990a) discusses the concept of a synthetic wheel (Figure 1.6d) that simultaneously performs a biped-like walk and a wheel-like roll on account of having arc-shaped feet whose radius of curvature equals the leg length. At each step, the swing foot lands just ahead of the stance foot to virtually synthesize the effect of a rolling wheel. But like the LIPM, this model also maintains a constant CoM height. McGeer also proposed a couple of models having heavy legs without and with knees (Figure 1.6e,f). These models get closer to accurately describing bipedal gaits albeit with increased mathematical sophistication.

The Spring Loaded Inverted Pendulum (SLIP; Figure 1.6g) or the spring-mass model incorporates a compliance element so that the leg of the inverted pendulum is no longer a rigid member of fixed length. This model was originally used for describing ballistic gaits. By suitably adjusting the spring stiffness, Geyer (2005) used it to encompass walking as well.

\subsection{Thesis scope}

Wheeled systems are energy efficient on prepared surfaces like roads and tracks. Legged systems are capable of traversing different terrains but can be lossy. At low speeds and on off-road surfaces, legged systems using dynamic walking can be energy efficient. Towards this objective, the dynamics of the walker needs to be modelled and controlled. In addition, the braking and ground impact losses need to be minimized. This thesis 


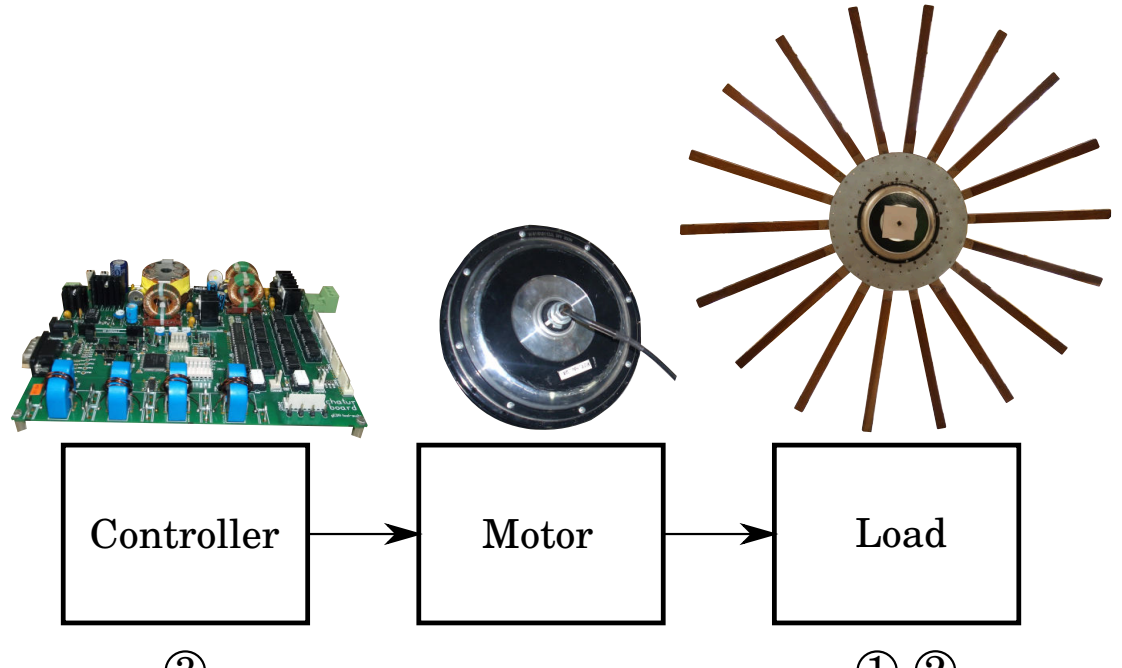

(3)

(1) (2)

Figure 1.7: Scope of the thesis. Numbers denote the problems addressed (see text below).

presents analysis and experiments on the dynamics and control of a rimless-spoked-wheel based mobile robot (Chatur) that belongs to a category between wheeled and legged systems.

The thesis addresses the following problems:

(1) Modelling the natural load dynamics and energetics of a rimless wheel based 2D dynamic walker and achieving sustained forward motion with pulsed torque actuation of varying pulse width.

(2) Investigating the physical constraints that impose fundamental limits on the choice of operating points of a generic inverted pendulum walker and locating optimal operating points.

(3) Developing a control topology for pulsed torque actuation of a rimless wheel based dynamic walker with its characteristic cyclic variations in reflected load torque and with wheel synchronization.

\subsection{Thesis outline}

Chapter 2 discusses the dynamics of a hub-actuated rimless wheel 'walking' on level ground. Various actuator torque profiles can be used to achieve dynamic walking of the rimless wheel. This chapter proposes a pulsed actuation torque giving rise to four torque regimes that achieve sustained walking and a fifth regime where the walker keeps slowing down with each step. The regimes are defined based on the relative magnitudes of energy 
losses and available actuator torque and can be identified based on the fraction of stance phase for which the actuator is energized. Theoretical analysis and experimental results are presented.

A simple closed-form analytical solution, using hyperbolic functions, is proposed for the stance phase inverted pendulum dynamics considering planar motion. Ground impacts are assumed to cause abrupt drop in velocity. A constant braking torque that lumps together the effect of several loss phenomena is also considered. Based on whether the CoM is rising or falling and whether or not there is an actuating torque, a stance phase can have four types of sub-phases - actuated rise, unactuated rise, actuated fall, unactuated fall. These are concatenated in four different ways to form repeating cycles yielding the four regimes. The experimental set-up is a fixed step-angle 2D dynamic walker named Chatur. It is constructed using two synchronized adjacent rimless wheels actuated at the hub using separate BLDC motors. Varying the magnitude and duration of the torque pulse, the proposed regimes are experimentally shown. The mechanical power consumption and cost of transport are computed from measured motor currents for different average forward speeds. Videos of the walks are also taken.

Chapter 3 investigates the choice of operating points for an inverted pendulum based bipedal dynamic walker in terms of constraints and optimality. The operating point of the walker can be specified by the combination of initial mid-stance velocity $\left(v_{0}\right)$ and step angle $\left(\phi_{m}\right)$ chosen for a given walk. Not all operating points lead to a realizable steadystate gait. In this chapter, using basic mechanics, a framework of physical constraints that limit the choice of operating points is proposed. The constraint lines thus obtained delimit the valid region of operation of the walker in the $v_{0}-\phi_{m}$ plane. Within this allowable region, sub-regions that result in various regimes of walking are identified.

A given average forward velocity $v_{x, a v g}$ can be achieved by several combinations of $v_{0}$ and $\phi_{m}$. Only one of these combinations results in the minimum mechanical power consumption and can be considered the optimum operating point for the given $v_{x, \text { avg }}$. This chapter proposes a method for obtaining this optimal operating point based on tangency of the power and velocity contours. Putting together all such operating points for various $v_{x, a v g}$, a family of optimum operating points, called the optimal locus, is obtained. For the energy loss and internal energy models chosen, the optimal locus obtained has a largely constant step angle with increasing speed but tapers off at non-dimensional speeds close to unity. Thus, choosing the right step angle and keeping it fixed over a broad range of speeds could lead to an inverted pendulum walker that is close to optimal from a 
mechanical energy perspective.

Chapter 4 elaborates the hardware design and proposes a control topology for pulsed torque actuation of Chatur, the rimless-wheel based mobile robot mentioned earlier. Pitfalls and caveats associated with reliable performance of the mechanical and electrical subsystems are pointed out. In order to ensure lateral stability, the system uses two contralateral wheels each driven by a separate BLDC hub motor. From a motor drive perspective, the mechanical load belongs to a unique class of dynamic loads whose reflected torque has a characteristic cyclic variation that repeats several times within a mechanical revolution.

The proposed control scheme has two hierarchical levels, an inner loop for torque control of BLDC motor implemented using a standard proportional-integral controller, and an outer loop for torque reference generation that uses the information on the ground impact instants and the motor position feedback. Ground impacts of the spokes are detected by an accelerometer to initiate the application of torque. The torque pulse magnitude can be set internally or by a manual operator via radio control. The pulse duration is programmable and enables attainment of various torque regimes at different steady state speeds. The wheels are synchronized so that corresponding spokes on both wheels move in unison. This is achieved by including a wheel synchronization loop that compensates for any lag between the wheels. Lag is detected based on number of sector changes in the hall-effect position sensor data received from both motors. An improved BLDC motor drive is developed wherein non-commutating current feedback is used to reduce current spikes during sector transitions. Experimental waveforms for controller validation are shown.

Chapter 5 summarizes the key research contributions made in the thesis and gives pointers for future work. 


\section{Chapter 2}

\section{Modeling of Dynamics and Torque Regimes}

Existing prototypes of the rimless wheel focus either on its passive 'walking' on a downward incline or on its multi-terrain capabilities. This chapter discusses the dynamics of a hub-actuated rimless wheel walking on level ground. Various actuator torque profiles can be used to achieve dynamic walking of the rimless wheel. A pulsed torque actuation is proposed giving rise to four torque regimes categorized by the pulse width range. A closed-form solution is proposed for the stance phase dynamics considering planar motion of an inverted pendulum (as an equivalent to a rimless spoked wheel with zero radius of gyration). Sustained forward motion spanning all four proposed regimes is achieved in an experimental prototype.

\subsection{Passive walking on level ground}

Consider a rimless wheel based walker, with stiff massless spokes, walking on level ground without any actuation. The ideal (lossless) and practical (lossy) cases are discussed in this section.

\subsubsection{Ideal case}

Figure 2.1a shows the free body diagram of the ideal rimless wheel walker. Only the spoke that is resting on the ground (stance leg) is shown making it an inverted pendulum. The 


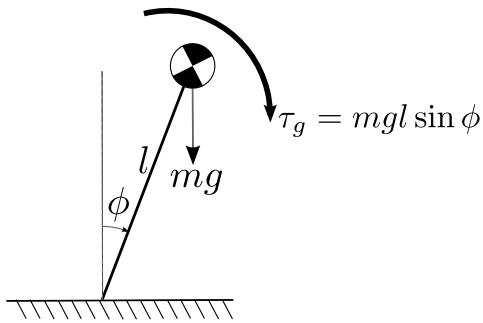

(a) Ideal: no braking, no actuation.

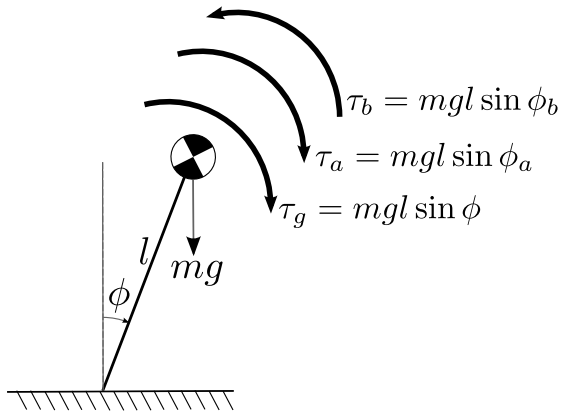

(b) Practical: with braking and actuation.

Figure 2.1: Free body diagram of a rimless wheel based walker of mass $m$, leg length $l$ and instantaneous fall angle $\phi$. Only the stance spoke is shown making it an inverted pendulum. $\tau_{g}, \tau_{a}$ and $\tau_{b}$ denote gravitational, actuator and braking torques.

other spokes are assumed to have no effect on the dynamics (until impact when the stance leg changes) and are hence not shown. Gravity applies a torque $m g l \sin \phi$ on the inverted pendulum about the foot point of the stance leg. The differential equation describing the dynamics over one stance phase is

$$
\frac{d^{2} \phi}{d t^{2}}-\frac{g}{l} \sin \phi=0
$$

The following constraints are used:

1. On account of having a constant inter-spoke angle $\left(2 \phi_{m}\right)$, the rimless wheel walker maintains a constant step angle $\left(\phi_{m}\right)$ and hence constant step length for the entire walk.

2. The walker is two-dimensional (2D) without any lateral dynamics i.e. the CoM moves only in the sagittal plane.

The walker starts moving from a vertical position having been imparted a finite CoM velocity $v_{0}$ (Figure $2.2 \mathrm{a}$ ). The initial energy $\left(E_{\text {init }}\right)$ imparted to the walker is hence $\frac{1}{2} m v_{0}^{2}$. As the inverted pendulum falls under the influence of gravity, the CoM traces a falling arc of radius equal to leg length $(l)$. It loses gravitational potential energy $\left(E_{p}\right)$ while gaining kinetic energy $\left(E_{k}\right)$. After falling through an angle $\phi_{m}$ (step angle or stance angle), the CoM velocity increases to $v_{m}$ (Figure $2.2 \mathrm{~b}$ ). If the transformation between gravitational potential energy and kinetic energy is lossless, conservation of mechanical energy yields

$$
v_{m}^{2}=v_{0}^{2}+2 g l\left(1-\cos \phi_{m}\right)
$$




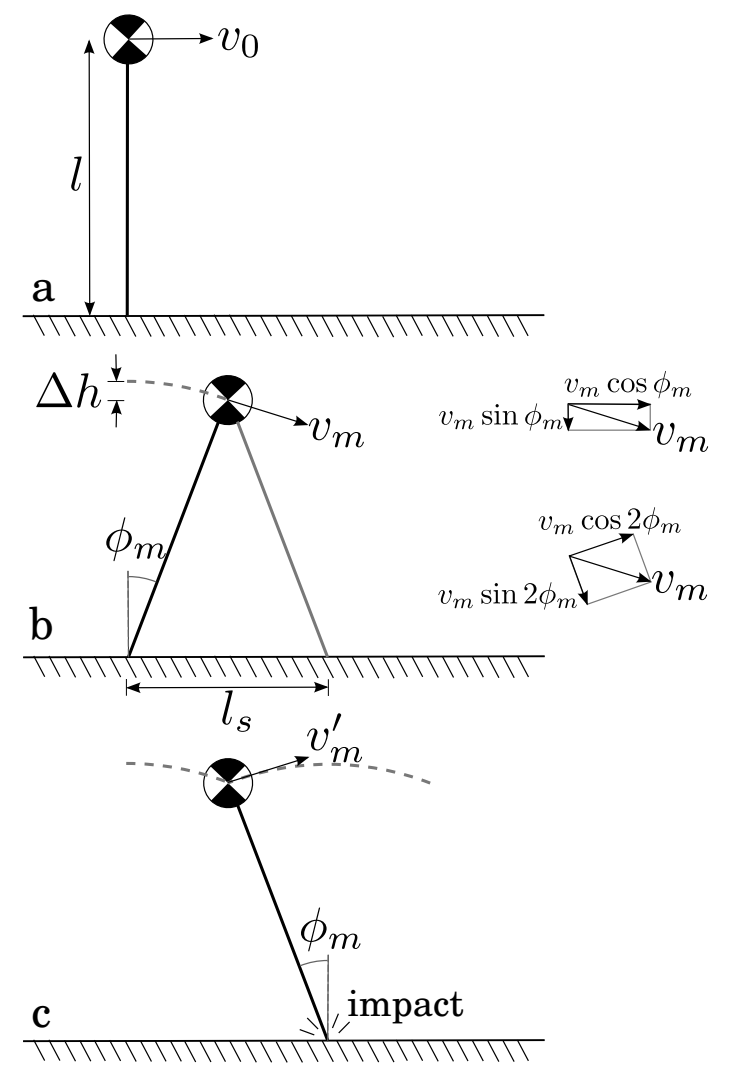

Figure 2.2: Forward motion of an inverted pendulum walker with (a) initial mid-stance velocity $v_{0}$, (b) step angle $\phi_{m}$, pre-impact velocity $v_{m}$, and (c) post-impact velocity $v_{m}^{\prime}$. The step length $\left(l_{s}\right)$ and CoM height variation $(\Delta h)$ are annotated. The CoM traces a trajectory consisting of a series of arcs of radius equal to the leg length $(l)$.

At this instant, the next leg strikes the ground and becomes the new stance leg (Figure 2.2c). If this ground impact were to be lossless, the CoM velocity $v_{m}$ gets instantaneously redirected along the arc corresponding to the new stance leg without any reduction in magnitude i.e. $v_{m}^{\prime}=v_{m}$. The CoM now traces a rising arc. The walker again reaches the mid-stance position at a CoM velocity $v_{0}$. This cycle can keep repeating indefinitely and steady forward motion is obtained without any net mechanical energy input to the system except at the start $\left(E_{\text {init }}\right.$; see Section 1.2). This ideal walker can execute passive walking on a level surface. Figure 2.3 plots, in black solid lines, the fall angle $\phi(t)$ and the CoM velocity $v(t)$ of this ideal walker. These are obtained by solving Equation 2.1 and are similar to the time domain waveforms presented in Pratt and Drakunov (2007). 


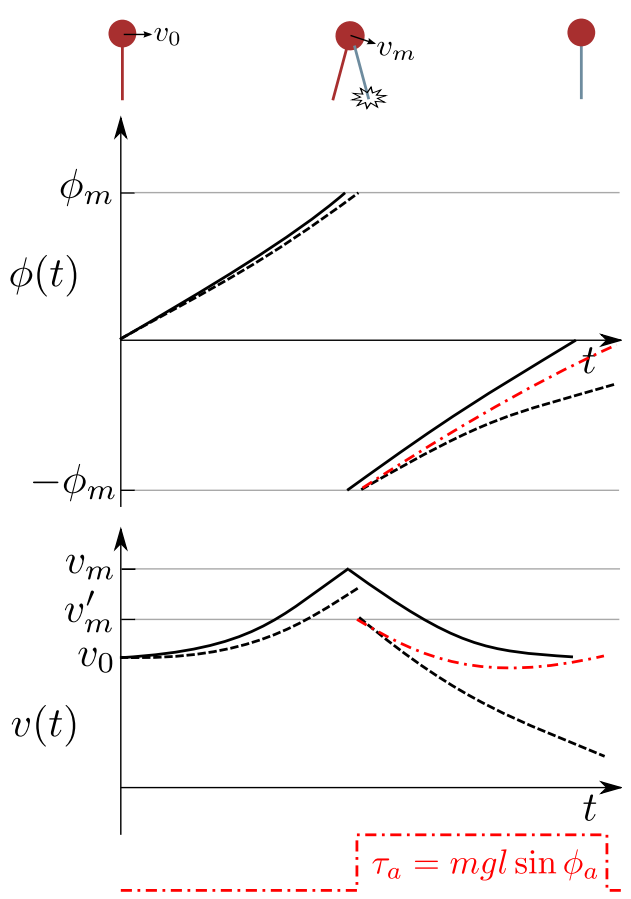

Figure 2.3: Fall angle $\phi(t)$ and CoM velocity $v(t)$ of ideal (black solid line) and practical (black dashed line) inverted pendulum walker on level ground, without actuation. Actuation (red dash dots) can put the system back to the state where it started $\left(v=v_{0}, \phi=0\right)$ thus enabling a sustained walk. The actuation torque may be present only for a portion of the stance phase.

\subsubsection{Practical case}

Practical walkers have braking as well as ground impact losses. This subsection models these two losses and discusses their effects on the dynamics and energetics of the walker.

\section{Braking loss}

Various phenomena that have a braking effect on the walker are listed below.

1. In human walking, some of the muscles perform negative work during the lowering of the CoM. They are fighting the fall dissipating energy as heat (Kunzig, 2001; Cavagna et al., 2002). Motors at the joints of a robotic walker can have opposing detent torques.

2. The active and passive rotary joints have Coulomb friction losses.

3. A finite sized foot pressing against the ground causes deformation in the foot as well as the ground. This results in a rolling resistance which can have a braking effect on the walker. 
The braking loss can be modeled by considering a continuous braking torque $\left(\tau_{b}\right)$ about the foot point. This torque lumps together the effects of all three phenomena mentioned above and continuously tries to slow down the walker. Strictly speaking, this torque need not be constant but assuming a constant braking torque is permissible because of two reasons: (i) the effect of braking torque on the dynamics of the walker is much smaller than that of the gravitational torque (and actuator torque, when present) and (ii) mathematical tractability. The braking torque is taken as

$$
\tau_{b}=m g l \sin \phi_{b}
$$

where $\phi_{b}$ is the braking torque parameter. Putting the braking torque in this form enables easy comparison with the instantaneous torque due to gravity $(m g l \sin \phi)$. It also facilitates an analytical solution for the dynamics of the walker (Section 2.3) with better insights.

As a result of braking loss, the transformation between gravitational potential energy and kinetic energy is not $100 \%$. The energy lost to braking as the walker falls through an angle $\phi_{m}$ from the vertical (half a stance phase) is given by

$$
\begin{aligned}
E_{b r k} & =(\text { braking torque }) \times(\text { angular displacement }) \\
& =\tau_{b} \phi_{m}=m g l \phi_{m} \sin \phi_{b}
\end{aligned}
$$

Conservation of energy gives an expression for the CoM velocity $v_{m}$ at the lowest potential energy position.

$$
v_{m}^{2}=v_{0}^{2}+2 g l\left(1-\cos \phi_{m}-\phi_{m} \sin \phi_{b}\right)
$$

With braking loss, the CoM velocity $v_{m}$ achieved at the end of a passive fall is lower than that for the ideal case (Equation 2.2). The differential equation describing the dynamics of one stance phase of this practical walker with braking loss can be derived as

$$
\frac{d^{2} \phi}{d t^{2}}-\frac{g}{l} \sin \phi+\frac{g}{l} \sin \phi_{b}=0
$$

\section{Impact loss}

In the absence of compliant elements to store and release the impact energy, the heel strike is lossy. It causes vibrations and heat dissipation resulting in an impulsive energy loss in the system. The kinetic energy at the start of the new stance phase is hence lesser 


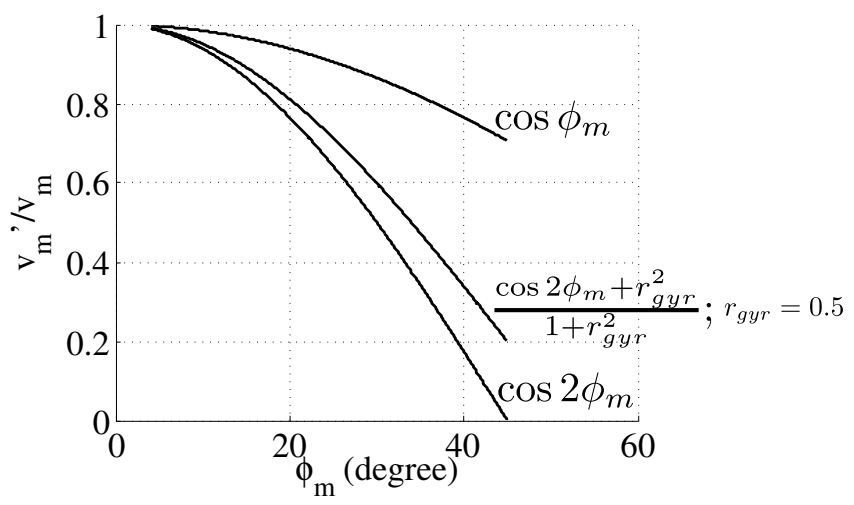

Figure 2.4: Ground impact loss models showing the drop in CoM velocity for various step angles. Higher $v_{m}^{\prime} / v_{m}$ implies lower losses.

than that at the end of the previous stance phase. The post-impact CoM velocity $v_{m}^{\prime}$ is less than $v_{m}$.

Alexander (2003) suggests that the vertical component of $v_{m}$ is completely lost on ground impact where as the horizontal component is completely retained (see Figure $2.2 \mathrm{~b}$ ). Hence, the kinetic energy lost on impact is

$$
E_{i m p}=\frac{1}{2} m v_{m}^{2} \sin ^{2} \phi_{m}
$$

The kinetic energy at the start of the new stance phase just after ground impact is computed as

$$
\frac{1}{2} m v_{m}^{\prime 2}=\frac{1}{2} m v_{m}^{2}-E_{i m p}=\frac{1}{2} m v_{m}^{2} \cos ^{2} \phi_{m}
$$

The post-impact CoM velocity simplifies to

$$
v_{m}^{\prime}=v_{m} \cos \phi_{m}
$$

In case of a rimless wheel with finite radius of gyration, McGeer (1990a) used conservation of angular momentum about the impact point to calculate the loss in speed.

$$
v_{m}^{\prime}=v_{m} \frac{\cos 2 \phi_{m}+r_{g y r}^{2}}{1+r_{g y r}^{2}}
$$

where $r_{g y r}$ is the radius of gyration normalized by leg length $l$. For $r_{g y r}=0$, the rimless wheel is equivalent to an inverted pendulum and the impact loss model in Equation 2.10 reduces to

$$
v_{m}^{\prime}=v_{m} \cos 2 \phi_{m}
$$

It is possible to arrive at Equation 2.11 by a different approach. Upon heel strike, the CoM cannot have any component of motion in the direction of the colliding leg because 
of the rigid leg assumption. Hence, the component of $v_{m}$ along the new stance leg is completely lost while the component perpendicular to it is unaffected (see Figure $2.2 \mathrm{~b}$ ). The kinetic energy lost on impact is

$$
E_{i m p}=\frac{1}{2} m v_{m}^{2} \sin ^{2} 2 \phi_{m}
$$

The kinetic energy at the start of the new stance phase is

$$
\frac{1}{2} m v_{m}^{\prime 2}=\frac{1}{2} m v_{m}^{2}-E_{i m p}=\frac{1}{2} m v_{m}^{2} \cos ^{2} 2 \phi_{m}
$$

Equation 2.13 yields a post-impact CoM velocity as given by Equation 2.11. Figure 2.4 plots the impact loss models described above (Equations 2.9-2.11) in terms of $v_{m}^{\prime} / v_{m}$ versus $\phi_{m}$. A higher value of $v_{m}^{\prime} / v_{m}$ indicates lower impact losses. Equation 2.11 is used in subsequent analysis of the dynamics of the walker.

Note that while braking loss occurs continuously, the ground impact loss is almost instantaneous*. Solving Equation 2.6 for the initial conditions $\phi_{i}=0, v_{i}=v_{0}$ yields the fall angle $\phi(t)$ and the CoM velocity $v(t)$ till heel strike. The impact loss causes an instantaneous change in state from $\left(\phi=\phi_{m}, v=v_{m}\right)$ to $\left(\phi=-\phi_{m}, v=v_{m}^{\prime}\right)$. Solving Equation 2.6 again for these new initial conditions gives the time evolution of $\phi(t)$ and $v(t)$ post impact.

Figure 2.3 plots, in black dashed lines, the fall angle $\phi(t)$ and the CoM velocity $v(t)$ of this lossy walker. Notice how the losses force the dynamics to slow down. If no measures are taken to replenish the energy lost to braking and impacts, this walker keeps slowing down till there is not enough energy to take the CoM to the highest potential energy position. The walker finally has to fall back instead of vaulting forward. This could happen in the next step or within a few steps, depending on the initial mid-stance velocity and the magnitude of the losses.

The total energy loss in one stance phase is given by

$$
\begin{aligned}
E_{\text {loss }} & =E_{b r k}+E_{i m p} \\
& =2 m g l \phi_{m} \sin \phi_{b}+\frac{1}{2} m v_{m}^{2} \sin ^{2} 2 \phi_{m}
\end{aligned}
$$

${ }^{*}$ Braking and ground impact losses are analogous to conduction and switching losses in semiconductor switches. 


\subsection{Sustained forward walk}

\subsubsection{Downward incline}

One way to achieve a sustained inverted pendulum type walk, is to make the passive walker go down a ramp that is at fixed angle $\gamma$ to the horizontal. The passive dynamic walkers mentioned in Section 1.3.2 use this approach. Introducing just the right amount of downward incline enables gravity to compensate for the impact and braking losses. The stance phase differential equation that describes this dynamics is given by

$$
\frac{d^{2} \phi}{d t^{2}}-\frac{g}{l} \sin (\phi+\gamma)+\frac{g}{l} \sin \phi_{b}=0
$$

If the slope is too small, gravity might not be able to compensate for the losses. The walker cannot have a sustained walk and eventually falls back. If the slope is too large, the walker keeps accelerating. Finally, the swing leg fails to catch up and be placed in time and the walker falls forward. A slope in between these two extremes enables the walker to passively walk down the slope at a nominally constant forward velocity determined by the slope angle.

\subsubsection{Torque actuation}

In the absence of a downward incline and in order to get a controllable average forward velocity, an actuator is required. Several passive-based dynamic walkers using actuators have been demonstrated (see Section 1.3.2). The actuator puts in just the right amount of mechanical energy in every stance phase to compensate for losses and puts the system back to the same state where it started thus enabling a sustained walk (see Figure 2.3, red dash dots). Various actuator torque profiles can be used to achieve a sustained walk. For the sake of simplicity, let the actuator apply a constant torque given by

$$
\tau_{a}=m g l \sin \phi_{a}
$$

where $\phi_{a}$ is the actuator torque parameter. As in the case of braking torque (Equation 2.3), Equation 2.16 expresses $\tau_{a}$ in a form similar to the torque due to gravity $(m g l \sin \phi)$.

In human walking, actuation is provided by the push-off of the trailing leg during the finite double support duration (Figure 2.5). The push-off force is a result of the calf muscles pulling the portion of the foot behind the ankle joint thus making the toe end of the foot push against the ground. The point of application of this force is about a step 

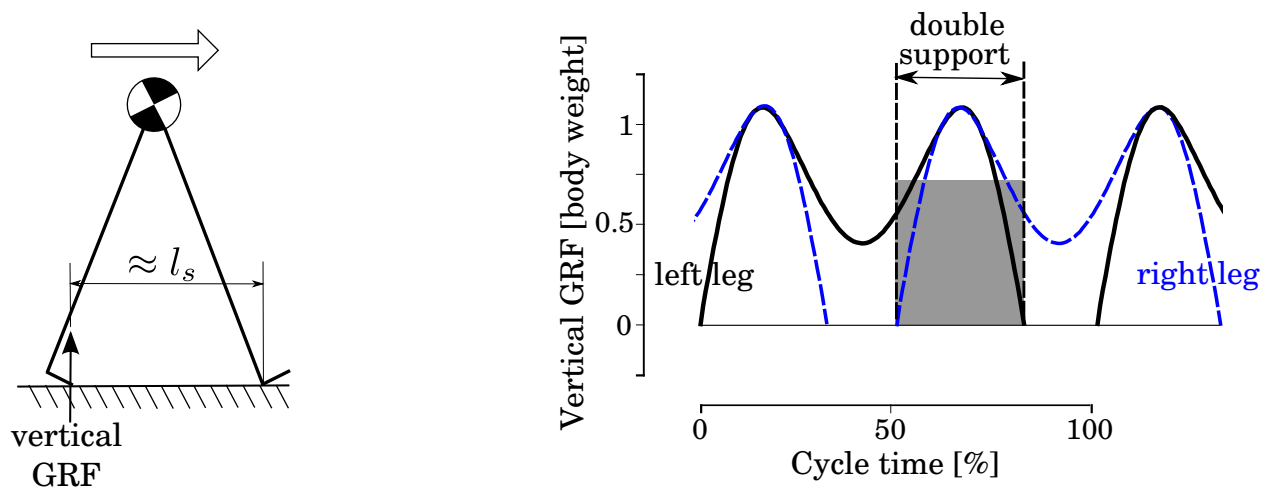

Figure 2.5: In human walking, the vertical component of ground reaction force (GRF) of the trailing leg during the double support duration provides the actuation torque: $\tau_{a} \approx$ (vertical GRF) $\times($ step length). Average value of the vertical GRF over the double support duration could be about $0.7 m g$. Adapted from Geyer (2005, chap. 1).

length $\left(l_{s}\right)$ away from the stance foot thus providing a mechanical advantage. It is capable of generating appreciable actuation torque on the system even with a modest magnitude of push-off force. Once the trailing leg leaves the ground there is no actuation on the system. Hence, the actuation can only be pulsed but not continuous. The actuation torque need not be constant valued. Equation 2.16 can approximate the effect of this varying actuator torque by using a value of $\tau_{a}$ equal to the average value of the torque for the double support duration which in turn is obtained from the average value of the vertical ground reaction force (GRF) during that duration (Figure 2.5).

Robotic walkers with actuated feet mimic the actuation method in humans and hence imbibe the merits and demerits discussed above. In a robotic walker without actuated feet, the motor that provides actuation could be at the hip joint as in case of a hubactuated rimless wheel. In such a case, there is no mechanical advantage and hence the motor torque requirement is higher. Actuation can be either pulsed or continuous depending on the fraction of the stance phase for which the motor is energized.

In presence of actuation, conservation of energy gives (compare with Equation 2.5)

$$
v_{m}^{2}=v_{0}^{2}+2 g l\left(1-\cos \phi_{m}+\phi_{m} \phi_{c}\right)
$$

where

$$
\phi_{c}=\sin \phi_{a}-\sin \phi_{b}
$$




\subsection{Analytical solution for the dynamics}

Incorporating braking and actuator torques, the free body diagram of the inverted pendulum is shown in Figure 2.1b. The stance phase dynamics of this practical inverted pendulum walker on level ground is formulated by

$$
\frac{d^{2} \phi}{d t^{2}}-\frac{g}{l} \sin \phi-\frac{g}{l} \sin \phi_{a}+\frac{g}{l} \sin \phi_{b}=0
$$

Equation 2.19 is a non-linear differential equation and can be solved numerically. Using the small angle approximation $(\sin \phi \approx \phi$ for small $\phi$ ) gives us a mathematically tractable linear differential equation.

$$
\frac{d^{2} \phi}{d t^{2}}-\frac{g}{l} \phi-\frac{g}{l} \sin \phi_{a}+\frac{g}{l} \sin \phi_{b}=0
$$

The maximum step angle in humans is about $25^{\circ}$ which is not a small enough angle to warrant the use of the small angle approximation. So the analytical solution to Equation 2.20 can have appreciable error with respect to the exact numerical solution of Equation 2.19. Yet, it can provide insight on the relative effects of various parameters on the dynamics. Taylor and Thomas (2014, chap. 3) present an analytical solution for the inverted pendulum model. Their solution uses elliptic integrals and is hence not a closed-form solution. In this work, a simple closed-form analytical solution using hyperbolic functions is proposed.

It is assumed that the actuation is either continuous or, if pulsed, is initiated at the instant of ground impact. If the actuation is continuous, Equation 2.20 holds throughout the stance phase. If actuation is pulsed, the differential equation changes for the portion of the stance phase that is unactuated. The $(g / l) \sin \phi_{a}$ term vanishes for the unactuated duration. In order to obtain an analytical solution for Equation 2.20, separate sets of initial conditions are required for the actuated and unactuated sub-phases of a stance phase.

Let us assume the following generic initial conditions at time $t=0$ :

$$
\phi(t=0)=\phi_{i} \text { and }\left.\frac{d \phi}{d t}\right|_{t=0}=\Omega_{i}=\frac{v_{i}}{l}
$$

For the above initial conditions, the analytical solution to Equation 2.20 gives an expression for the fall angle as a function of time:

$$
\phi(t)=\frac{v_{i}}{v_{n}} \sinh \left(\omega_{n} t\right)+\left(\phi_{c}+\phi_{i}\right) \cosh \left(\omega_{n} t\right)-\phi_{c}
$$



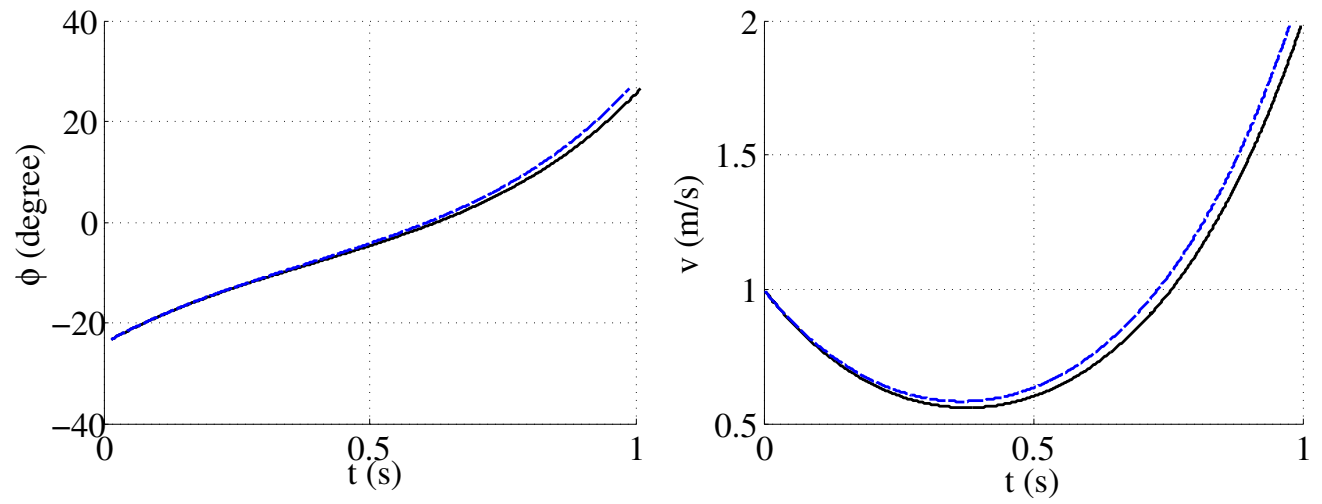

Figure 2.6: Comparison of analytical (solid) and numerical (dashed) solutions for inverted pendulum dynamics. Parameter values: $m=50 \mathrm{~kg}, l=1 \mathrm{~m}, \phi_{b}=5^{\circ}$ and $\phi_{a}=15^{\circ}$. Initial conditions: $\phi_{i}=-25^{\circ}$ and $v_{i}=1 \mathrm{~m} / \mathrm{s}$. Final condition: $\phi_{f}=25^{\circ}$.

where $v_{n}, \omega_{n}$ and $\phi_{c}$ (Equation 2.18) are constants.

$$
\begin{aligned}
& v_{n}=\sqrt{g l} \\
& \omega_{n}=\sqrt{\frac{g}{l}}
\end{aligned}
$$

The instantaneous angular velocity of the inverted pendulum is given by:

$$
\Omega(t)=\frac{d \phi}{d t}=\frac{v_{i}}{l} \cosh \left(\omega_{n} t\right)+\left(\phi_{c}+\phi_{i}\right) \cdot \omega_{n} \cdot \sinh \left(\omega_{n} t\right)
$$

The magnitude of the instantaneous CoM velocity is given by:

$$
v(t)=\Omega(t) l=v_{i} \cosh \left(\omega_{n} t\right)+\left(\phi_{c}+\phi_{i}\right) \cdot v_{n} \cdot \sinh \left(\omega_{n} t\right)
$$

The forward velocity of the CoM is the horizontal component of the above velocity.

$$
v_{x}(t)=v(t) \cos \phi
$$

In order to compare the above analytical solution with the numerically computed exact solution, consider an inverted pendulum with $m=50 \mathrm{~kg}$ and $l=1 \mathrm{~m}$. Consider an exaggerated stance phase ranging from $\phi_{i}=-25^{\circ}$ to $\phi_{f}=25^{\circ}$ with initial velocity $v_{i}=1 \mathrm{~m} / \mathrm{s}$. Let $\phi_{b}=5^{\circ}$ and $\phi_{a}=15^{\circ}$ where the actuation is present throughout the stance phase. The analytical solution (Equation 2.22 and 2.26) slowly keeps diverging from the exact solution of Equation 2.19 when both are given the same initial conditions (Figure 2.6). In this worst case, where the stance leg sweeps through an angle of $50^{\circ}$ ( $25^{\circ}$ before and after mid-stance), the analytical solution is about $6 \%$ higher than the numerical solution. For typical fall angles lesser than $25^{\circ}$, the error is smaller. 
Unlike the above extreme case, generally the actuation is present only for a portion of the stance phase. If the walker starts from the vertical position with an initial mid-stance velocity $v_{0}$ as in Section 2.1, the initial conditions are

$$
\phi_{i}=0 \text { and } v_{i}=v_{0}
$$

Take $\phi_{a}=0$ for this unactuated fall. Substituting these into Equations 2.22-2.27 gives the complete dynamics for the unactuated fall. In the absence of braking loss (i.e. $\phi_{b}=0$ ), the fall angle would vary as a sine hyperbolic function and the angular velocity (and CoM velocity) as a cosine hyperbolic function. At the instant of ground impact, the actuator is energized to give a constant torque (Equation 2.16). The CoM velocity instantaneously changes to $v_{m}^{\prime}$ as given by Equation 2.11. For the subsequent actuated rise of the CoM, the initial conditions are

$$
\phi_{i}=-\phi_{m} \text { and } v_{i}=v_{m}^{\prime}
$$

Again substituting these into Equations 2.22-2.27 gives the complete dynamics for the actuated rise (and fall).

\subsection{Torque regimes}

A nominally periodic sequence of steps can be realised only if the actuator keeps putting in the right amount of energy. To achieve a sustained walk, the energy put in by the actuator during the time that it is energized should compensate for the losses incurred in one stance phase (Equation 2.14). Let the actuator be energized for the duration that the inverted pendulum sweeps an angle $\Delta \phi$ from $\phi=-\phi_{m}$ to $\phi=-\phi_{m}+\Delta \phi$. For a given actuator torque, $\Delta \phi$ works out to

$$
\Delta \phi=\frac{E_{\text {loss }}}{\tau_{a}}
$$

The spatial duty ratio $\left(d_{i s}\right)$ of the actuator torque (current) can be defined as

$$
d_{i s}=\frac{\text { angular displacement when actuated }}{\text { angular displacement in one stance phase }}=\frac{\Delta \phi}{2 \phi_{m}}
$$

Based on the value of $\Delta \phi$ obtained in Equation 2.30, there are five possibilities.

Case 1: $\tau_{a}>\frac{E_{\text {loss }}}{\phi_{m}}, \Delta \phi<\phi_{m}, d_{i s}<0.5$

Actuator torque is high enough to be removed before mid-stance (Figure 2.7a). 


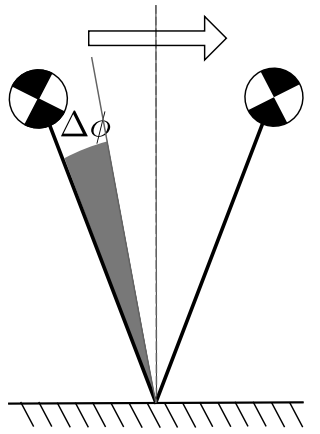

(a)

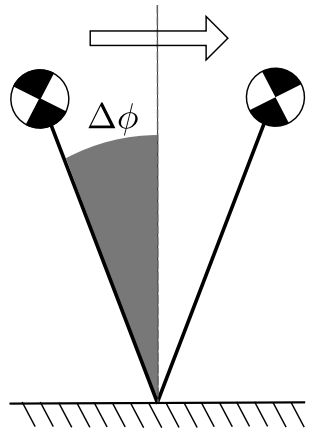

(b)

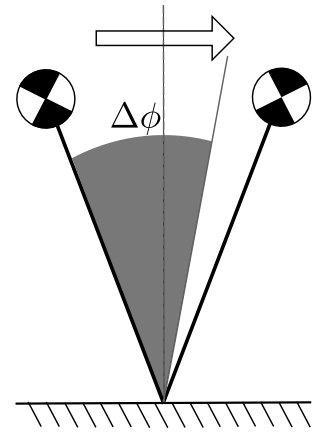

(c)

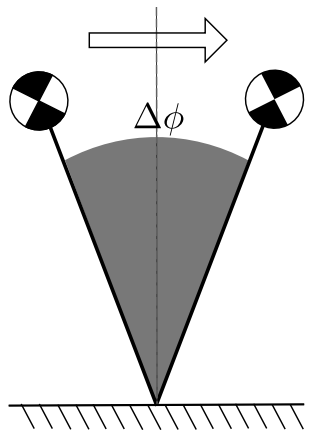

(d)

Figure 2.7: Portion of stance phase for which actuation is present in an inverted pendulum walker. (a) Regime 1, $\Delta \phi<\phi_{m}$, (b) Regime 2, $\Delta \phi=\phi_{m}$, (c) Regime 3, $\phi_{m}<\Delta \phi<2 \phi_{m}$, (d) Regime $4, \Delta \phi=2 \phi_{m}$.

Case 2: $\tau_{a}=\frac{E_{\text {loss }}}{\phi_{m}}, \Delta \phi=\phi_{m}, d_{i s}=0.5$

Actuator torque is just enough to be removed at mid-stance (Figure 2.7b).

Case 3: $\frac{E_{\text {loss }}}{2 \phi_{m}}<\tau_{a}<\frac{E_{\text {loss }}}{\phi_{m}}, \phi_{m}<\Delta \phi<2 \phi_{m}, 0.5<d_{i s}<1$

Actuator torque needs to be removed after mid-stance but before next heel-strike (Figure $2.7 \mathrm{c})$.

Case 4: $\tau_{a}=\frac{E_{\text {loss }}}{2 \phi_{m}}, \Delta \phi=2 \phi_{m}, d_{i s}=1$

Actuator torque needs to be applied continuously to sustain a steady walk (Figure $2.7 \mathrm{~d}$ ).

Case 5: $\tau_{a}<\frac{E_{\text {loss }}}{2 \phi_{m}}, \Delta \phi>2 \phi_{m}, d_{i s}>1$

Actuator torque is insufficient to sustain a steady walk even when applied continuously unless the step angle is higher for subsequent steps.

Consider the parameters as indicated in Table 2.1. For a given $\phi_{m}, v_{0}$ can be varied to obtain all five cases described above. Figures 2.8-2.10 plot the fall angle, CoM velocity, mechanical energy and actuator torque of the inverted pendulum walker for Case 1Case 5 (computed using the analytical solutions obtained in Section 2.3). Each case is annotated with the sub-phases marked in terms of whether the CoM is rising or falling and whether or not there is any actuation in that sub-phase. There are four types of subphases - actuated rise (AR), unactuated rise (UR), actuated fall (AF), unactuated fall (UF). The rise time of the CoM is generally larger than its fall time. But with reasonably high actuator torques, it is possible to achieve almost equal rise and fall times. 
Table 2.1: Parameter values used for the torque regime waveforms (Figures 2.8-2.10).

\begin{tabular}{cc}
\hline Parameter & Value \\
\hline$m$ & $65 \mathrm{~kg}$ \\
$g$ & $9.81 \mathrm{~m} / \mathrm{s}^{2}$ \\
$l$ & $1 \mathrm{~m}$ \\
$\phi_{a}$ & $10^{\circ}$ \\
$\phi_{b}$ & $1^{\circ}$ \\
\hline
\end{tabular}

Case 1-Case 4 delineate the four possible torque regimes for sustained walking of the practical inverted pendulum walker. In regimes 1 and 2, the repeating limit cycle is achieved within the second mid-stance. The mid-stance velocity is the same for all steps. Regimes 3 and 4 achieve limit cycle within the second ground impact. The second mid-stance velocity is lower than the first one, but is maintained at that lower value for all subsequent mid-stances. Humans mostly exhibit regime 1 limit cycle walking where the actuation due to push-off is present only for a short duration at the beginning of a stance phase. Sections 2.5-2.6 describe the construction and working of a rimless wheel based walker that exhibits all four torque regimes.

Case 5 leads to a fifth regime that does not yield a sustained walk within the second ground impact. It results in the walker slowing down to lower and lower mid-stance velocities at each subsequent step till the losses equal the energy put in by the actuator. A steady state regime 4 walk could thus be reached in a matter of few steps. If the actuator torque is too low, the walker keeps slowing down till it falls back and stops walking. Note that once fall back happens, the direction of the braking torque reverses. So the term corresponding to braking torque in Equation 2.20 flips sign and the expression for the analytical solution changes.

Figure 2.11 plots the state space trajectories (phase portraits) of the five regimes on the $\phi-v$ plane. The grey lines depict phase portraits of the ideal inverted pendulum walker. Since it has no losses, each grey line represents a particular mechanical energy level. In presence of losses, the walker keeps falling to lower energy levels. The task of the actuator is to restore the system back to the original energy level thus enabling a cyclic trajectory. This 'home' energy level is defined by the combination of $v_{0}$ and $\phi_{m}$ chosen. The repeating cycles, obtained by suitable concatenation of the sub-phases, are annotated above each of the phase portraits in Figure 2.11. Regime 5 does not yield repeating cycles as seen in the phase portrait. 
Each of the sub-phase durations $\left(T_{s p}\right)$ is a non-linear function of many variables and can be derived using Equation 2.22 and the initial and final conditions for that sub-phase. Taking the generic final condition as $\phi=\phi_{f}$, a sub-phase duration can be deduced to

$$
T_{s p}=\frac{\sinh ^{-1} z}{\omega_{n}}
$$

where

$$
\begin{aligned}
z & =\frac{-B+\sqrt{B^{2}-4 A C}}{2 A} \\
A & =\frac{v_{i}^{2}}{v_{n}^{2}}-\left(\phi_{c}+\phi_{i}\right)^{2} \\
B & =-2\left(\phi_{c}+\phi_{f}\right) \frac{v_{i}}{v_{n}} \\
C & =\phi_{f}^{2}-\phi_{i}^{2}-2 \phi_{c}\left(\phi_{i}-\phi_{f}\right)
\end{aligned}
$$

For any of the four regimes of sustained walking, the stance phase duration $(T)$ is obtained by adding the durations of all the sub-phases involved. The step frequency $(f)$ is the reciprocal of the stance phase duration. 

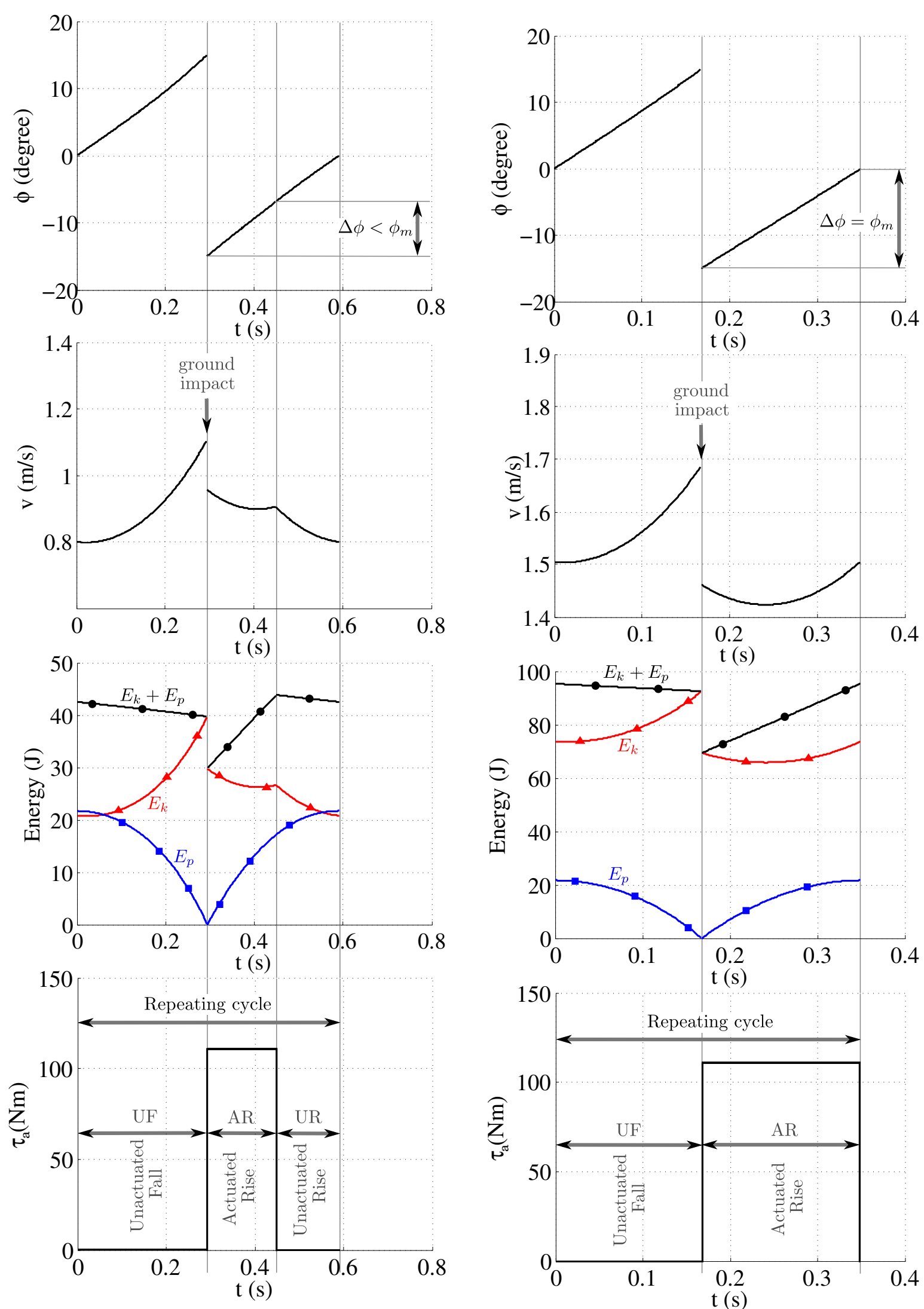

(a) Regime 1 walking: $v_{0}=0.8 \mathrm{~m} / \mathrm{s}, \phi_{m}=15^{\circ}$ (b) Regime 2 walking: $v_{0}=1.5 \mathrm{~m} / \mathrm{s}, \phi_{m}=15^{\circ}$

Figure 2.8: Regime 1 and regime 2 walking 

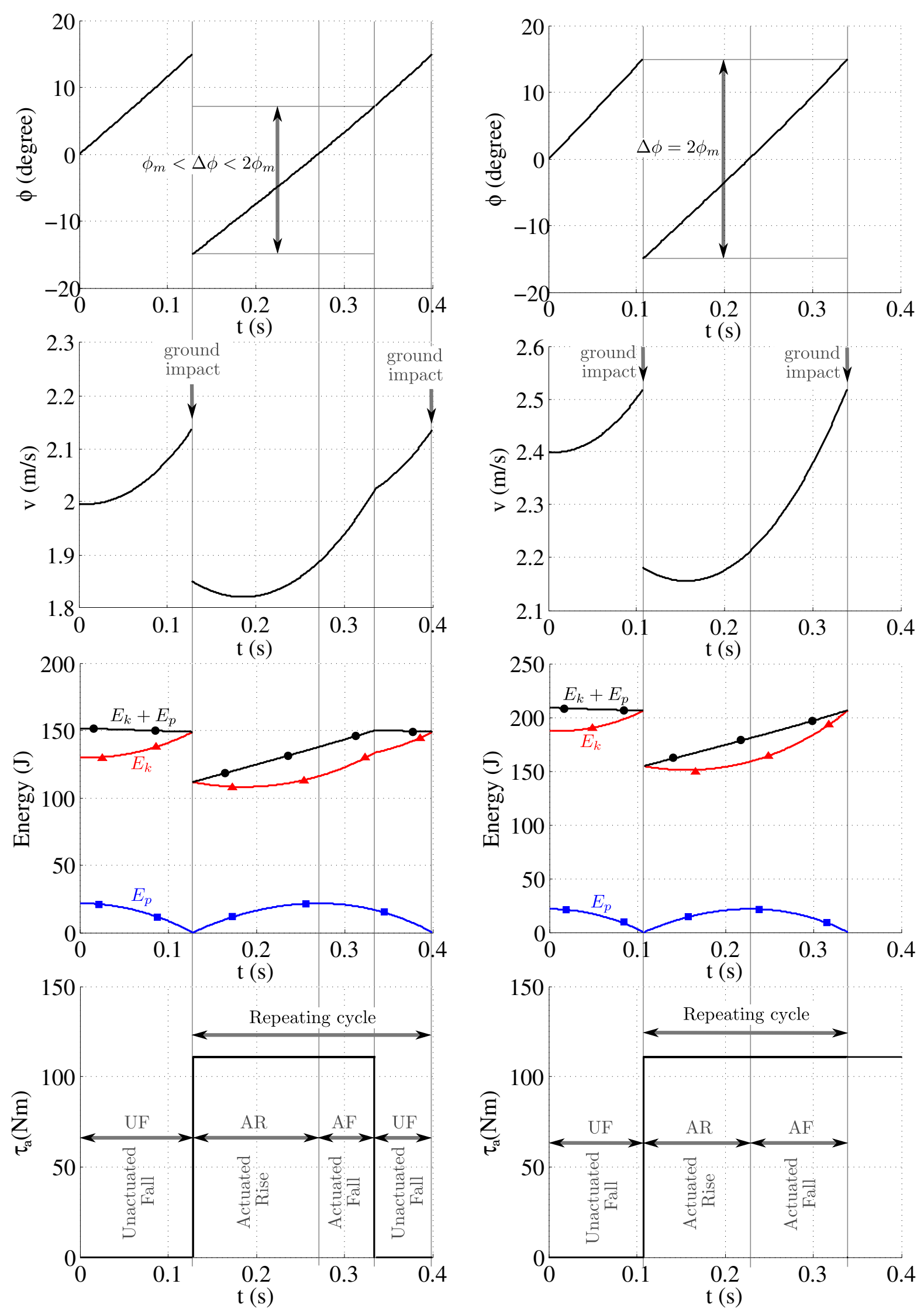

(a) Regime 3 walking: $v_{0}=2 \mathrm{~m} / \mathrm{s}, \phi_{m}=15^{\circ}$

(b) Regime 4 walking: $v_{0}=2.4 \mathrm{~m} / \mathrm{s}, \phi_{m}=15^{\circ}$

Figure 2.9: Regime 3 and regime 4 walking 

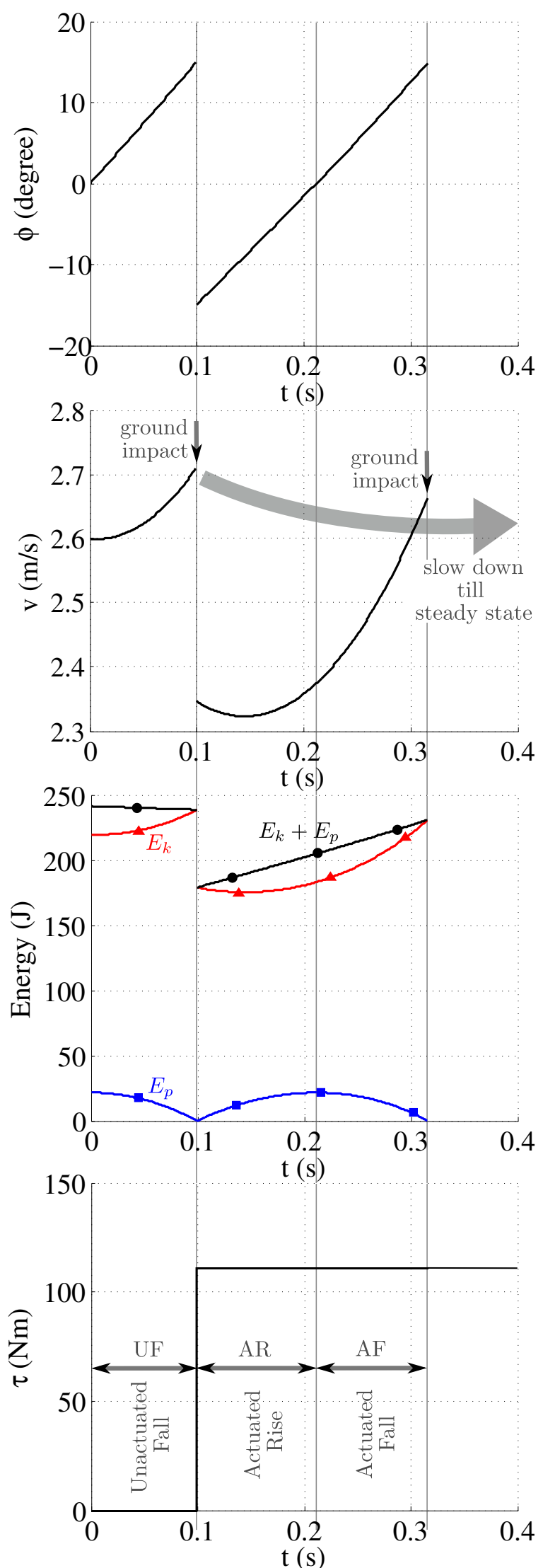

Figure 2.10: Regime 5 walking: $v_{0}=2.6 \mathrm{~m} / \mathrm{s}, \phi_{m}=15^{\circ}$ 


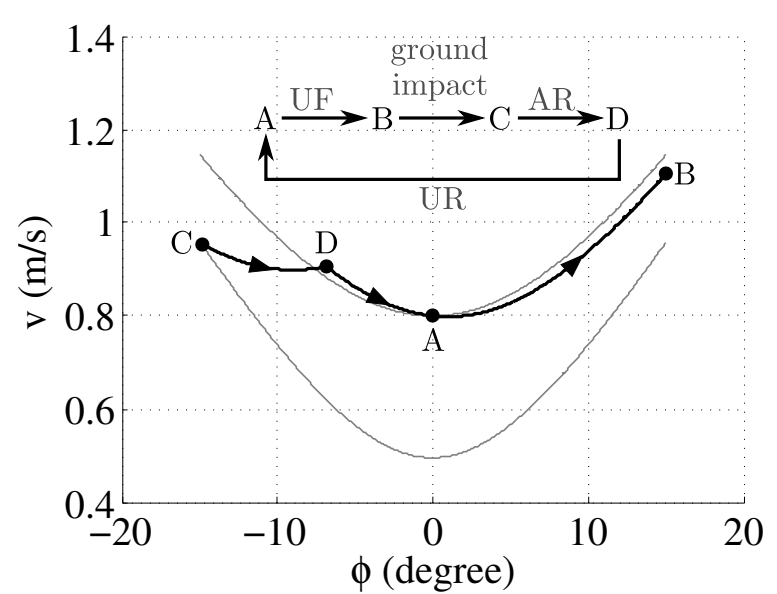

(a) Regime 1 walking: $v_{0}=0.8 \mathrm{~m} / \mathrm{s}, \phi_{m}=15^{\circ}$

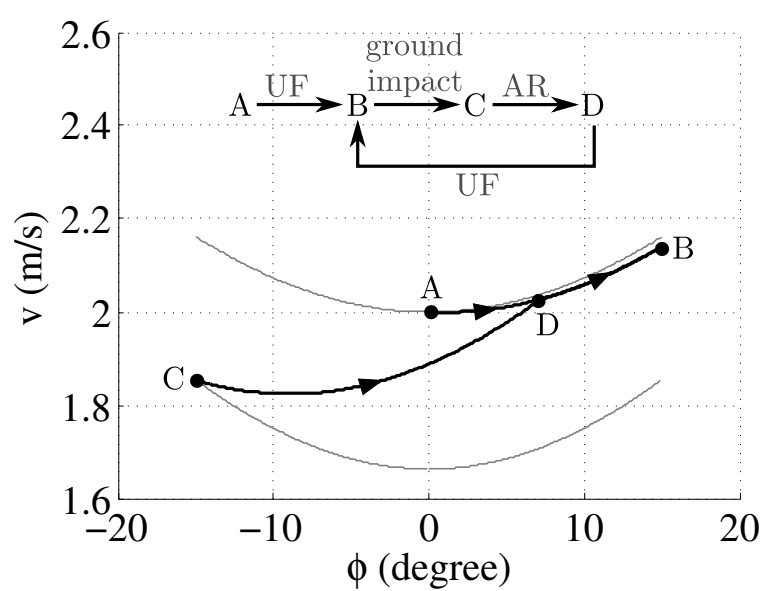

(c) Regime 3 walking: $v_{0}=2 \mathrm{~m} / \mathrm{s}, \phi_{m}=15^{\circ}$

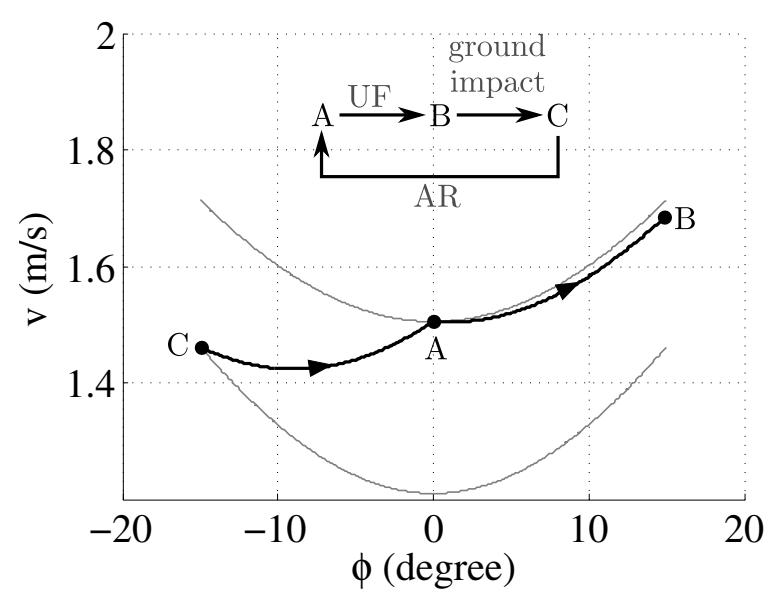

(b) Regime 2 walking: $v_{0}=1.5 \mathrm{~m} / \mathrm{s}, \phi_{m}=15^{\circ}$

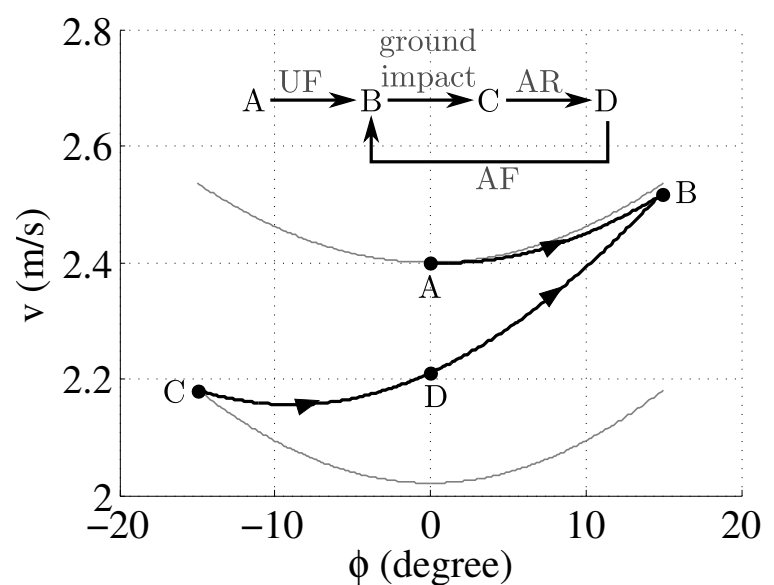

(d) Regime 4 walking: $v_{0}=2.4 \mathrm{~m} / \mathrm{s}, \phi_{m}=15^{\circ}$

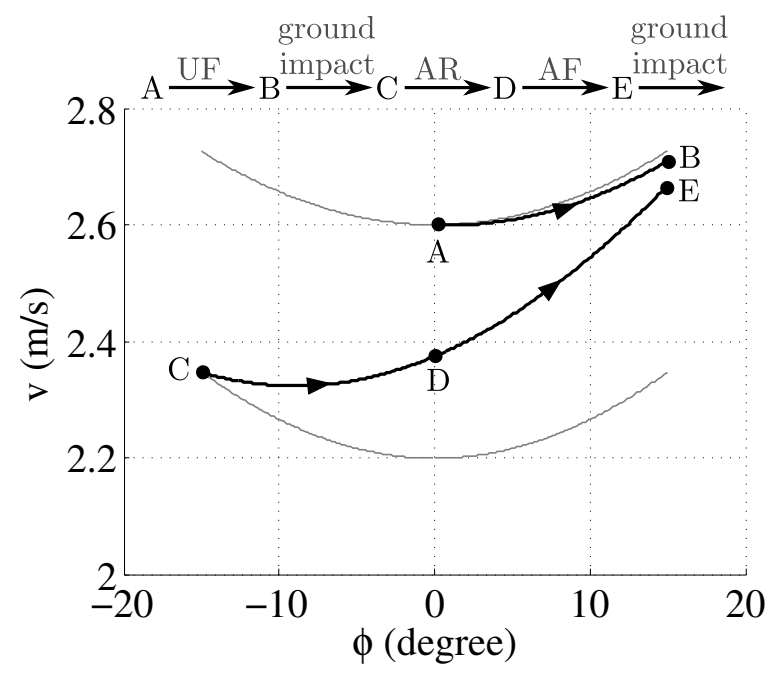

(e) Regime 5 walking: $v_{0}=2.6 \mathrm{~m} / \mathrm{s}, \phi_{m}=15^{\circ}$

Figure 2.11: Phase portraits for all five torque regimes corresponding to Figures 2.8-2.10. 


\subsection{Experimental prototype}

Chapter 4 gives a detailed description of the experimental prototype (a rimless wheel based 2D walker named Chatur) and the control scheme used for pulsed torque control of the actuators. A brief outline is given here.

\subsubsection{Hardware}

Figure 4.9 shows the experimental prototype built. Chatur is a fixed step-angle 2D dynamic walker implemented using the concept of rimless spoked wheel. Lateral stability is ensured by using two contralateral rimless wheels. The mechanical subsystem consists of the rimless wheels, a welded mild steel (MS) frame and enclosures for the electronics. No attempt is made to minimize weight, so that the heavy parts - MS frame and sealed lead acid (SLA) batteries - can emulate payload weight (refer Table 2.2 for part weights).

The electrical hardware consists of batteries, motors, and PCBs for power and control electronics. Each rimless wheel is actuated by a three-phase brushless dc (BLDC) hub motor with 18 equidistant wooden spokes attached to its external rotor. The BLDC motors are driven by three-phase inverters. The sensing, signal conditioning and control circuits reside in a controller card designed for Chatur. Table 2.2 specifies the parameters of the system to put it in context with the mathematical model. The rated motor current $\left(I_{\max }\right)$ is $12 \mathrm{~A}$ and the motor torque constant $\left(K_{t}\right)$ is $0.6 \mathrm{Nm} / \mathrm{A}$. Hence the maximum actuator torque parameter (combined effect of both motors driven by rated current) is given by

$$
\phi_{a, \max }=\sin ^{-1}\left(\frac{2 I_{\max } K_{t}}{m g l}\right) \approx 3^{\circ}
$$

This implies that if the motor is stuck at zero velocity at the lowest potential energy position $\left(\phi=\phi_{m}=10^{\circ}\right)$, it cannot raise itself. Owing to this torque limitation of the motor, initial raising of the center of mass is done manually and measurements are taken for steady state walks of Chatur. The braking torque parameter $\left(\phi_{b}\right)$ is only approximately known as it is difficult to separate the impact losses and braking losses in experimental measurements.

As the motor tries to rotate its rotor, there is a counter torque acting on the stator that is attached to the frame. In order to stop this counter torque from rotating the frame instead of the rimless wheels, a tail piece is added. The end of the tail has a castor wheel that rests on the ground. The ground reaction force acting on this castor helps resist the 
Table 2.2: Specifications of Chatur.

\begin{tabular}{ll}
\hline Parameter & Value \\
\hline Leg length, $l$ & $0.57 \mathrm{~m}$ \\
\hline Step angle, $\phi_{m}$ & $10^{\circ}$ \\
\hline Step length, $l_{s}=2 l \sin \phi_{m}$ & $0.2 \mathrm{~m}$ \\
\hline CoM height variation, $\Delta h=l\left(1-\cos \phi_{m}\right)$ & $9 \mathrm{~mm}$ \\
\hline Mass, $m$ & rimless wheels, $2 \times 12 \mathrm{~kg}$ \\
& frame, $7 \mathrm{~kg}$ \\
& batteries, $15 \mathrm{~kg}$ \\
& others, $4 \mathrm{~kg}$ \\
& total, $50 \mathrm{~kg}$ \\
\hline Motor torque constant, $K_{t}$ & $0.6 \mathrm{Nm} / \mathrm{A}$ \\
\hline Maximum actuator torque parameter, & $3^{\circ}$ \\
$\phi_{a, m a x}$ (at $I_{\text {max }}=12$ A) & \\
\hline Braking torque parameter, $\phi_{b}$ & $\sim 1^{\circ}$ \\
\hline
\end{tabular}

counter torque on the frame. A design without the need for a tail piece and castor wheel is also possible. The OutRunner from Robotics Unlimited uses a counterweight, placed away from the axle, on the frame to oppose the counter torque (Robotics-Unlimited, 2014).

\subsubsection{Control scheme}

The torque of the BLDC hub motor is controlled in a pulsed manner. Torque control is achieved using a Proportional-Integral (PI) controller for the motor current. Ground impacts are detected by an accelerometer to initiate the application of constant torque. The magnitude and duration of the applied torque pulse is programmable by the user and enables attainment of various regimes at different steady state speeds. The magnitude of the torque pulse is set by a manual operator via radio control. The rimless wheels need to be synchronized so that corresponding legs on both wheels move in unison without a lag between one another. This is achieved by adding an extra term in the torque reference that compensates for any lag sensed between the rimless wheels. Lag is detected based 


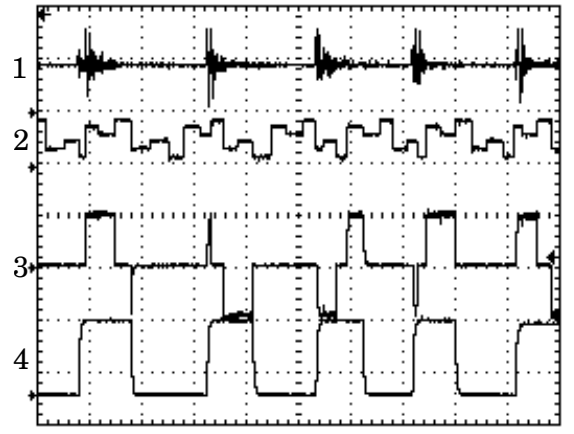

(a) $v_{x, a v g}=0.37 \mathrm{~m} / \mathrm{s}$.

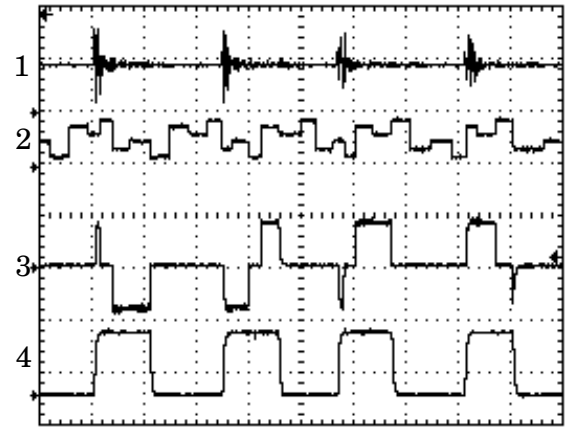

(b) $v_{x, a v g}=0.33 \mathrm{~m} / \mathrm{s}$.

Figure 2.12: Experimental waveforms for regime 1 walking: (1) Accelerometer output (2 $\mathrm{V} /$ div), (2) Motor-1 sector number, (3) Motor-1 phase current $I_{a 1}(10 \mathrm{~A} / \mathrm{div})$, (4) Torque reference $(1 \mathrm{~V} /$ div $)$, time scale $250 \mathrm{~ms} / \operatorname{div}, d_{i s}=3 / 8$.

on number of sector changes in the position sensor data received from both motors.

The rotor position is determined by a 3-bit digital output from a hall effect position sensor that is built into the motor. The sensor gives six different position outputs (sector numbers) per electrical cycle. This translates to a mechanical position resolution of $2.4^{\circ}$ for the 25 pole pair motor. The sequence of sector numbers for counter-clockwise (CCW) rotation is 5-4-6-2-3-1 (see experimental waveforms in Section 2.6). This is used for the right motor on Chatur. The reverse sequence gives clockwise (CW) rotation and is used for the left motor.

\subsection{Experimental results}

This section details the experimental results obtained with Chatur. Since the set-up is mobile, all waveforms are captured using a battery-operated four-channel oscilloscope mounted on Chatur (Figure 4.9). Waveforms for all four torque regimes are depicted. For each set of waveforms, average power and average velocity calculations are done based on known system parameters (see Table 2.2). Videos of the walks are also taken.

\subsubsection{Torque Regimes}

At the instant of ground impact (detected by an impulse in the accelerometer output), the torque reference is raised to a value set by the operator. The torque reference is brought back to zero after a predefined number of sector changes following the ground impact. 


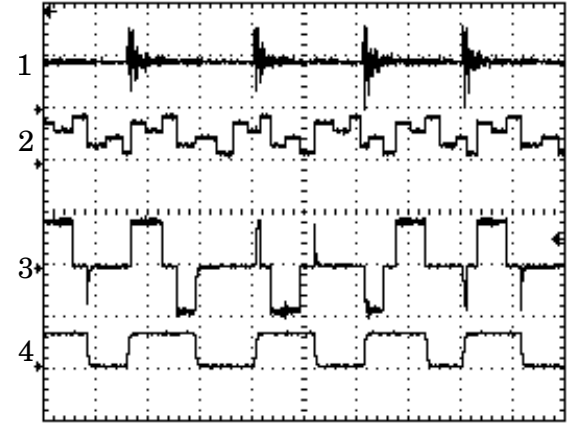

(a) $v_{x, a v g}=0.38 \mathrm{~m} / \mathrm{s}$.

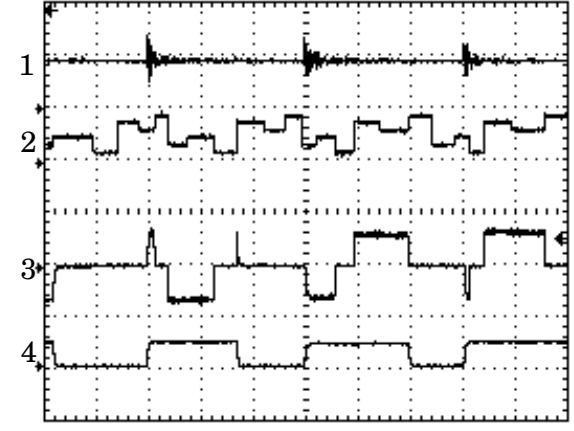

(b) $v_{x, a v g}=0.27 \mathrm{~m} / \mathrm{s}$.

Figure 2.13: Experimental waveforms for regime 2 walking: (1) Accelerometer output (2 V/div), (2) Motor-1 sector number, (3) Motor-1 phase current $I_{a 1}$ (10 A/div), (4) Torque reference $(2 \mathrm{~V} / \mathrm{div})$, time scale $250 \mathrm{~ms} / \mathrm{div}, d_{i s}=4 / 8$.

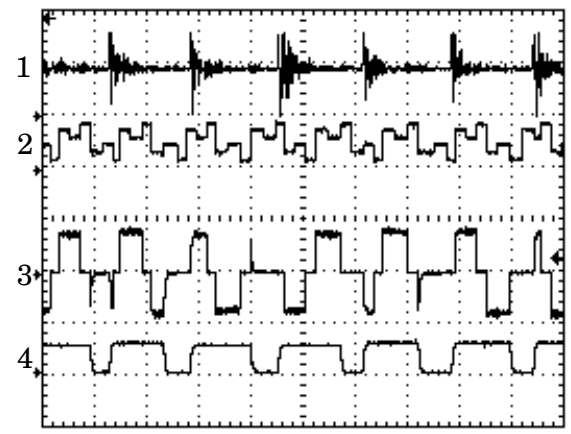

(a) $v_{x, a v g}=0.49 \mathrm{~m} / \mathrm{s}$.

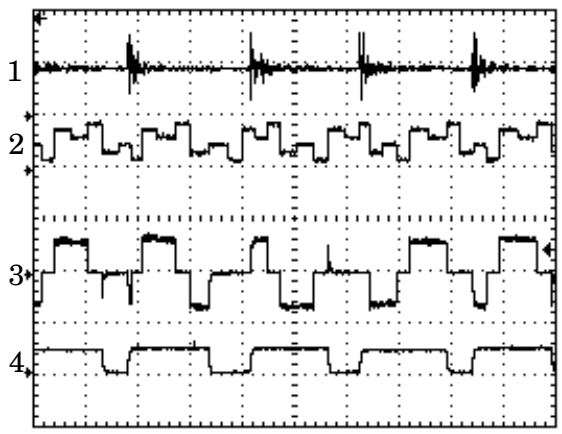

(b) $v_{x, a v g}=0.36 \mathrm{~m} / \mathrm{s}$.

Figure 2.14: Experimental waveforms for regime 3 walking: (1) Accelerometer output (2 V/div), (2) Motor-1 sector number, (3) Motor-1 phase current $I_{a 1}(10 \mathrm{~A} /$ div), (4) Torque reference $(2 \mathrm{~V} / \mathrm{div})$, time scale $250 \mathrm{~ms} / \operatorname{div}, d_{i s}=5 / 8$.

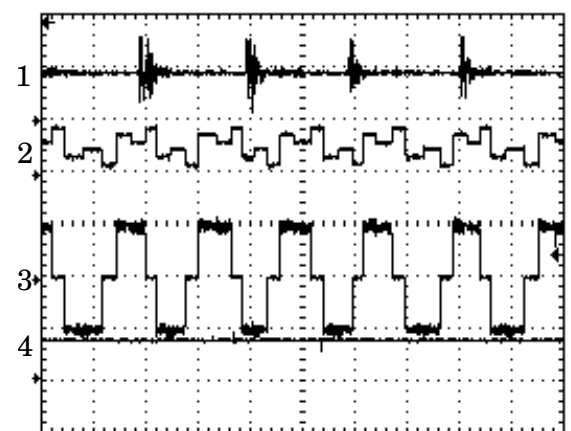

(a) $v_{x, a v g}=0.39 \mathrm{~m} / \mathrm{s}$.

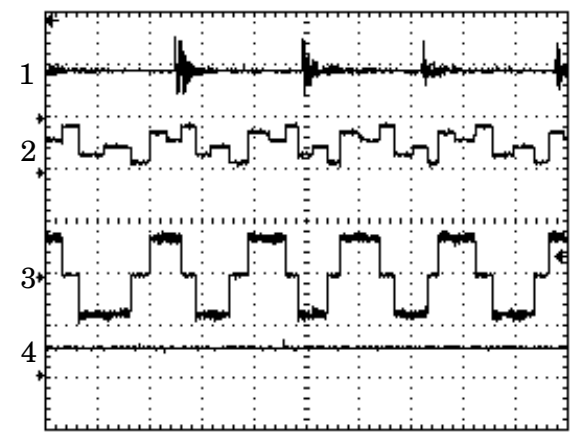

(b) $v_{x, a v g}=0.33 \mathrm{~m} / \mathrm{s}$.

Figure 2.15: Experimental waveforms for regime 4 walking: (1) Accelerometer output (2 V/div), (2) Motor-1 sector number, (3) Motor-1 phase current $I_{a 1}$ (5 A/div), (4) Torque reference $(1 \mathrm{~V} /$ div $)$, time scale $250 \mathrm{~ms} / \mathrm{div}, d_{i s}=1$. 
All waveforms and measurements correspond to nominally steady state walks on cement floor. The four torque regimes (Figures 2.12-2.15) are achieved by suitable choice of the magnitude and duty ratio of the applied torque. Only one of the motor phase currents is shown. Note that the sector dwell times around the ground impact instants are smaller than those away from the impacts. This is because the CoM speed and hence the motor speed is higher at the lowest potential energy position. Waveforms for two operating points of each regime are shown. Several such operating points are taken to carry out the power calculations.

The spatial duty ratio $\left(d_{i s}\right)$ of the torque is varied from $3 / 8$ to 1 . The motor speed is not constant during a stance phase and the rise and fall time of the CoM can also be unequal. Hence, the spatial duty ratio can translate to a slightly different temporal duty ratio $\left(d_{i}\right)$ as seen in the waveforms. The torque magnitude is varied by changing the motor current over the range 3-10 A.

Regime 1 walking is achieved only at higher torques (for $I>8 \mathrm{~A}$ ) and hence not many operating points are obtained. Even for a given speed, not all ground impacts have same losses because of practical asymmetries in step angles and non-uniform nature of foot points and surface of travel. A walk that is nominally in regime 2 keeps going to regimes 1 and 3 every now and then owing to these asymmetries. Similarly, a walk that is nominally in regime 4 keeps going to regimes 3 and 5 every now and then.

If the applied torque is too low, fall back can happen following a particularly lossy ground impact. Once fall back occurs, Chatur cannot raise itself owing to the torque limitation discussed in Section 2.5.1. At higher speeds, the ground impacts can be quite severe leading to large shocks on the rimless wheels that are capable of damaging it. Thus the range of achieved average speeds is limited at both ends.

\subsubsection{Calculation of average power and average velocity}

For a given set of waveforms obtained in any given regime, the average torque in a motor is given by

$$
\tau_{\text {avg }}=K_{t} \cdot I \cdot d_{i}
$$

where $K_{t}$ is the motor torque constant, $I$ is the magnitude of the current pulse and $d_{i}$ is the duty ratio (temporal) of the current pulse. The average time interval between consecutive impacts gives the average stance phase duration (or average step duration, $T$ ). 


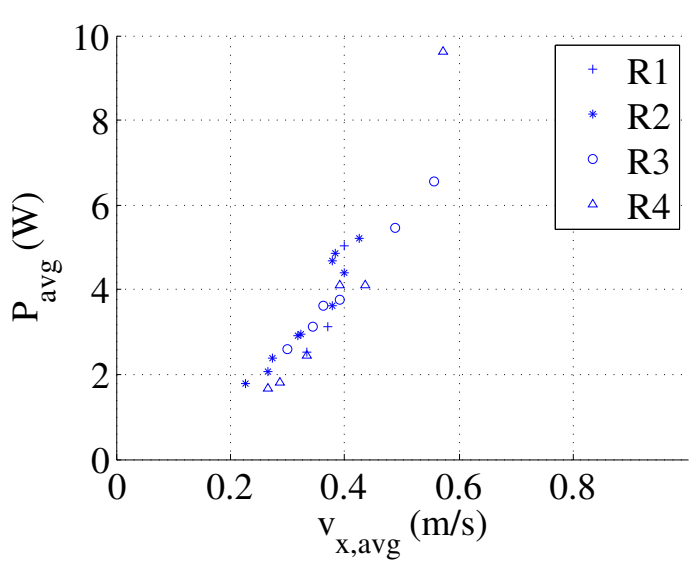

Figure 2.16: Average mechanical power versus average forward velocity.

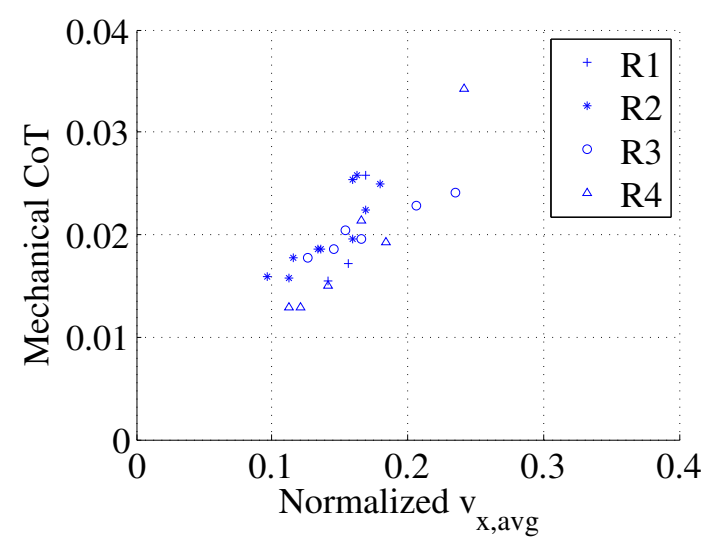

Figure 2.17: Mechanical cost of transport versus normalized average forward velocity.

For step length $l_{s}$, the average forward velocity is computed as

$$
v_{x, a v g}=\frac{l_{s}}{T}
$$

For leg length $l$, the average angular velocity of the rimless wheels is computed as

$$
\omega_{\text {avg }}=\frac{v_{x, a v g}}{l}
$$

The average mechanical power including both motors is then calculated as

$$
P_{a v g}=2 \cdot \tau_{a v g} \cdot \omega_{a v g}
$$

These calculations are carried out for various operating points (Table 2.3). The scatter plot of average power versus average velocity is shown in Figure 2.16. Non-dimensionalizing both quantities, the scatter plot for mechanical CoT $\left(P_{a v g} / m g v_{x, a v g}\right)$ versus normalized velocity $\left(v_{x, a v g} / \sqrt{g l}\right)$ is shown in Figure 2.17. The scatter plots use separate markers for the different regimes as indicated in the legend. R1-R4 denote regimes 1-4.

\subsubsection{Discussion}

Though the experimental set-up is built to emulate the mathematical model described in Sections 2.1-2.4, there are bound to be some deviations in practice. The 'foot' i.e. the end of a spoke is a finite sized rectangle $(33 \mathrm{~mm} \times 25 \mathrm{~mm})$ as opposed to being a point. This affects the CoM trajectory and hence its total vertical excursion $(\Delta h)$ in a stance phase. The type of foot - its shape, size and compliance — plays a key role in determining the ground impact losses. The losses are likely to be more complex than the simplistic models chosen (Equation 2.14). The loss parameters are not constant and have 
Table 2.3: Measurements from experimental waveforms.

\begin{tabular}{|c|c|c|c|c|c|c|}
\hline$I(\mathrm{~A})$ & $T(\mathrm{~s})$ & $d_{i}$ & $\tau_{a v g}(\mathrm{Nm})$ & $v_{x, a v g}(\mathrm{~m} / \mathrm{s})$ & $\omega_{\text {avg }}(\mathrm{rad} / \mathrm{s})$ & $P_{a v g}(\mathrm{~W})$ \\
\hline \multicolumn{7}{|c|}{ Regime $1, d_{i s}=3 / 8$} \\
\hline 10 & 0.50 & 0.60 & 3.60 & 0.40 & 0.70 & 5.1 \\
\hline 10 & 0.54 & 0.40 & 2.40 & 0.37 & 0.65 & 3.1 \\
\hline 8 & 0.60 & 0.45 & 2.16 & 0.33 & 0.58 & 2.5 \\
\hline \multicolumn{7}{|c|}{ Regime $2, d_{i s}=4 / 8$} \\
\hline 10 & 0.47 & 0.58 & 3.48 & 0.43 & 0.75 & 5.2 \\
\hline 10 & 0.53 & 0.59 & 3.54 & 0.38 & 0.66 & 4.7 \\
\hline 10 & 0.52 & 0.60 & 3.60 & 0.38 & 0.67 & 4.9 \\
\hline 9 & 0.50 & 0.58 & 3.13 & 0.40 & 0.70 & 4.4 \\
\hline 8 & 0.53 & 0.57 & 2.74 & 0.38 & 0.66 & 3.6 \\
\hline 7 & 0.73 & 0.59 & 2.48 & 0.27 & 0.48 & 2.4 \\
\hline 7 & 0.62 & 0.62 & 2.60 & 0.32 & 0.57 & 2.9 \\
\hline 7 & 0.88 & 0.53 & 2.23 & 0.23 & 0.40 & 1.8 \\
\hline 7 & 0.63 & 0.62 & 2.60 & 0.32 & 0.57 & 2.9 \\
\hline 6 & 0.75 & 0.61 & 2.20 & 0.27 & 0.47 & 2.1 \\
\hline \multicolumn{7}{|c|}{ Regime $3, d_{i s}=5 / 8$} \\
\hline 8 & 0.36 & 0.70 & 3.36 & 0.56 & 0.97 & 6.5 \\
\hline 7.5 & 0.41 & 0.71 & 3.20 & 0.49 & 0.86 & 5.5 \\
\hline 6.5 & 0.51 & 0.70 & 2.73 & 0.39 & 0.69 & 3.8 \\
\hline 6.5 & 0.55 & 0.73 & 2.85 & 0.36 & 0.64 & 3.6 \\
\hline 6 & 0.67 & 0.69 & 2.48 & 0.30 & 0.52 & 2.6 \\
\hline 6 & 0.58 & 0.72 & 2.59 & 0.35 & 0.61 & 3.1 \\
\hline \multicolumn{7}{|c|}{ Regime $4, d_{i s}=8 / 8=1$} \\
\hline 8 & 0.35 & 1.00 & 4.80 & 0.47 & 1.00 & 9.6 \\
\hline 5 & 0.51 & 1.00 & 3.00 & 0.39 & 0.69 & 4.1 \\
\hline 4.5 & 0.46 & 1.00 & 2.70 & 0.43 & 0.76 & 4.1 \\
\hline 3.5 & 0.60 & 1.00 & 2.10 & 0.33 & 0.58 & 2.5 \\
\hline 3 & 0.70 & 1.00 & 1.80 & 0.29 & 0.50 & 1.8 \\
\hline 3 & 0.75 & 1.00 & 1.80 & 0.27 & 0.47 & 1.7 \\
\hline
\end{tabular}


wide variations in a practical scenario. There is a lot of distributed mass in the entire set-up as opposed to a lumped mass at the axle. The rimless wheels have some mass distributed away from the center. This results in a non-zero radius of gyration $\left(r_{g y r}\right)$ of the wheel thus requiring some internal energy $\left(E_{i n t}\right)$ to rotate it as well as altering the inverted pendulum dynamics. The heavy batteries are hanging below the axle taking the CoM of the system a few centimeters below the axle. The castor wheel (not present in mathematical model) faces some rolling resistance and can alter the dynamics. All these are non-idealities that can be further considered to extend the proposed model.

\subsection{Conclusion}

This chapter proposed the existence of four torque regimes in a rimless wheel based 2D walker actuated at the hub by pulsed torque. The four regimes are categorized based on the relative magnitudes of energy losses and actuator torque which in turn dictates the portion of the stance phase for which actuation is required. A closed-form analytical solution for the 2D dynamics of an inverted pendulum walker is presented which is also applicable for the rimless wheel with zero radius of gyration. An experimental prototype called Chatur is built using two contralateral rimless wheels and the four regimes are experimentally validated. The mechanical power as well as cost of transport is computed for all operating points. Pulsed torque of constant magnitude has been considered in this work. More generic walkers can have time varying actuator torques. But the basic idea of actuation being present over a certain angular displacement of the stance leg still holds. Hence the proposed torque regimes with the defined repeating cycles can be observed in all walkers - engineered or biological. 


\section{Chapter 3}

\section{Analysis of Operating Point Space}

Taking mass, leg length, braking torque and available actuator torque as fixed parameters, the operating point of an inverted pendulum walker can be specified by the combination of initial mid-stance velocity $\left(v_{0}\right)$ and step angle $\left(\phi_{m}\right)$ chosen for a given walk. The analysis in this chapter builds on the inverted pendulum model and investigates its implications on the choice of operating points for a bipedal walker. Using basic mechanics, Section 3.1 proposes a framework of physical constraints that define the valid region of operating points. Section 3.2 proposes an optimal locus of operating points (within the valid region) that tries to minimize the mechanical power expended for a given forward velocity.

\subsection{Constraints and limits}

Not all combinations of initial mid-stance velocity $v_{0}$ and step angle $\phi_{m}$ are realizable. This section explains all the physical constraints that limit the region of valid operating points for the inverted pendulum walker.

\subsubsection{Take-off constraint}

If the bob of an inverted pendulum moves too fast, it no longer stays on the circular trajectory shown in Figure 2.2c i.e. it takes off. Usherwood (2005) argues that even when the mid-stance speed equals $\sqrt{g l}$, at larger fall angles the CoM is bound to go away from its nominal trajectory (arc of radius equal to leg length). This is because as the angle increases, the component of gravity along the leg reduces while the velocity and 


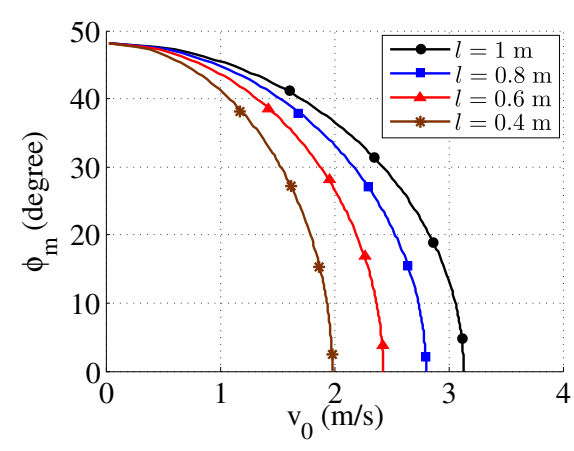

(a) $\phi_{b}=0$

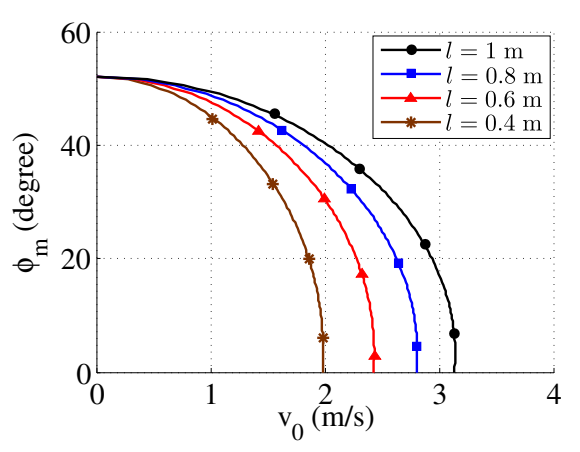

(b) $\phi_{b}=5^{\circ}$

Figure 3.1: Take-off constraint lines for various leg lengths and no actuation $\left(\phi_{a}=0\right)$. (a) is a reproduction of the plots in Usherwood (2005) mapped to the $v_{0}-\phi_{m}$ plane.

hence the required centripetal acceleration increases. Accordingly, the take-off constraint can be specified in terms of the maximum possible step angle for any average forward velocity (Usherwood, 2005; Usherwood et al., 2008). Take-off happens when centripetal acceleration equals the component of gravity along the stance leg.

$$
\frac{v^{2}}{l}=g \cos \phi
$$

In the limiting case, take-off happens right at the end of the fall i.e. at $\phi=\phi_{m}$. At that instant, the centripetal acceleration is given by

$$
\frac{v_{m}^{2}}{l}=g \cos \phi_{m}
$$

The take-off constraint for an ideal lossless inverted pendulum can be obtained by substituting Equation 3.2 in Equation 2.2. Substituting instead in Equation 2.5 gives the take-off constraint line in presence of braking.

$$
v_{0}^{2}=g l\left(3 \cos \phi_{m}+2 \phi_{m} \sin \phi_{b}-2\right)
$$

Figure 3.1a plots the take-off constraint lines for various values of $l$ for the lossless unactuated fall. Figure 3.1b plots the same with braking loss included. Operating points below these constraint lines guarantee no take-off. Actuation has not been considered here as the fall of the CoM is typically unactuated. In case of an actuated fall, the take-off constraint lines will move lower down.

There is another equivalent interpretation of the take-off constraint. As the inverted pendulum falls, the compression in the leg keeps reducing. At the instant the compression becomes zero, the ground will need to start pulling the leg. This is not possible (unless the foot is stuck on the ground due to some adhesive or suction pads) and hence the inverted pendulum takes off. 


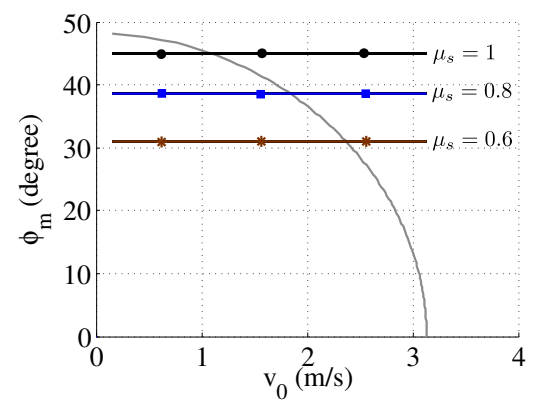

Figure 3.2: Sliding constraint lines for various values of coefficient of static friction $\left(\mu_{s}\right)$.

\subsubsection{Sliding constraint}

Taking large steps (large step angles) can lead to sliding. This is why one takes smaller steps while walking on a slippery surface like ice or wet floor. The static friction force is proportional to the vertical component of the ground reaction force. Since the rod is massless, the ground reaction force $\left(F_{g r}\right)$ acting at the bottom of the rod equals the compressive force $\left(F_{c}\right)$ in the rod because of the weight of the bob pressing on it

$$
F_{g r}=F_{c}=m g \cos \phi-\frac{m v^{2}}{l}
$$

As the inverted pendulum falls, the vertical component of the ground reaction force keeps decreasing while the horizontal component keeps increasing. At the instant when the horizontal component equals the maximum static friction available, sliding occurs. In the limiting case, the maximum step angle equals that at which sliding occurs.

$$
\begin{array}{ll}
F_{g r} \sin \phi_{m} & =\mu_{s} F_{g r} \cos \phi_{m} \\
\Rightarrow F_{g r}=0 & \text { or } \quad \phi_{m}=\tan ^{-1} \mu_{s}
\end{array}
$$

where $\mu_{s}$ is the coefficient of static friction between the foot point and the ground. Zero ground reaction force is equivalent to zero compression in the leg and has already been dealt with as the take-off constraint. Hence, the sliding constraint is formulated by

$$
\phi_{m}=\tan ^{-1} \mu_{s}
$$

Figure 3.2 shows the sliding constraint lines for a few different values of $\mu_{s}$. They are superimposed on the take-off constraint line (no braking) for reference. Operating points below the sliding constraint line ensure no sliding. 


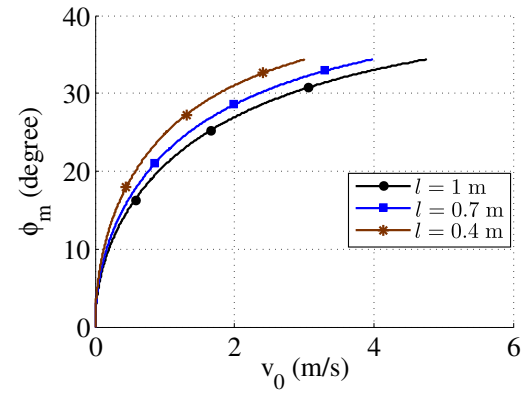

(a) $\phi_{a}=0, \phi_{b}=0$

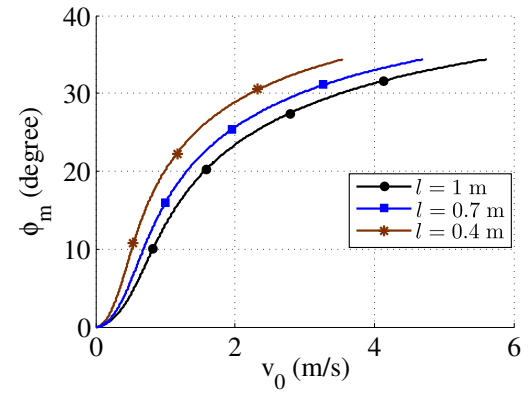

(b) $\phi_{a}=0, \phi_{b}=5^{\circ}$

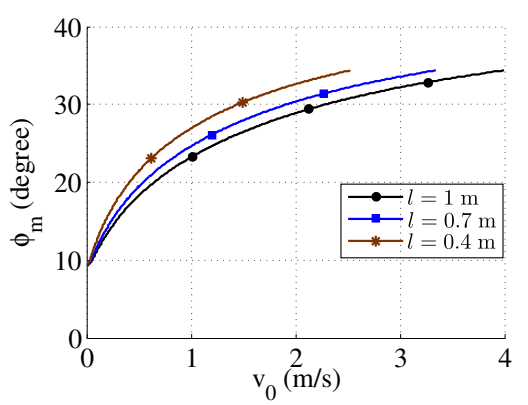

(c) $\phi_{a}=10^{\circ}, \phi_{b}=5^{\circ}$

Figure 3.3: Fall back constraint lines for various leg lengths.

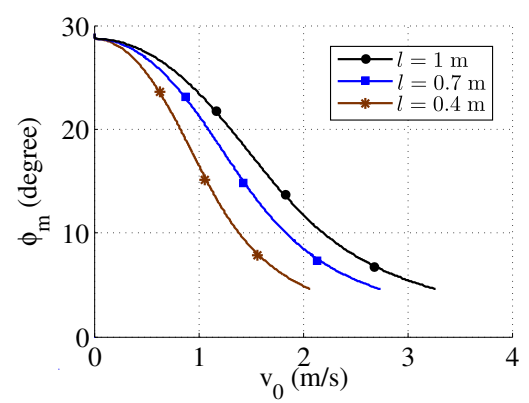

(a) $\phi_{a}=5^{\circ}, \phi_{b}=0$

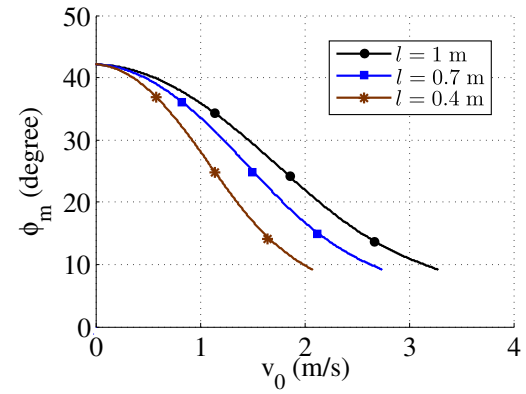

(b) $\phi_{a}=10^{\circ}, \phi_{b}=0^{\circ}$

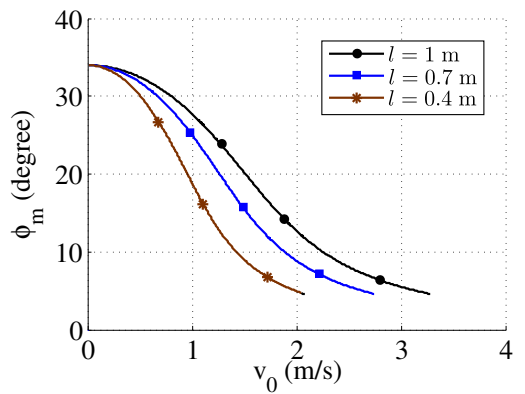

(c) $\phi_{a}=10^{\circ}, \phi_{b}=5^{\circ}$

Figure 3.4: Steady-state constraint lines for various leg lengths.

\subsubsection{Fall-back constraint}

The inverted pendulum walker just averts falling backwards if, following a ground impact, the inverted pendulum reaches mid-stance at zero CoM velocity. In this limiting case, application of conservation of energy gives

$$
\begin{gathered}
\frac{1}{2} m v_{m}^{\prime 2}+\phi_{m} m g l \sin \phi_{a}-\phi_{m} m g l \sin \phi_{b}=m g l\left(1-\cos \phi_{m}\right) \\
v_{m}^{\prime 2}=2 g l\left(1-\cos \phi_{m}-\phi_{m} \phi_{c}\right)
\end{gathered}
$$

Using Equations 2.5, 2.11 and 3.8, the fall back constraint is obtained in terms of $v_{0}$ and $\phi_{m}$. It is plotted for a few different values of parameters in Figure 3.3. Choosing operating points below these constraint lines makes sure that the walker does not fall back.

\subsubsection{Steady-state constraint}

Given an actuator torque, certain combinations of $v_{0}$ and $\phi_{m}$ lead to a scenario where the losses are too high for the actuator to compensate within one stance phase (Case 5 


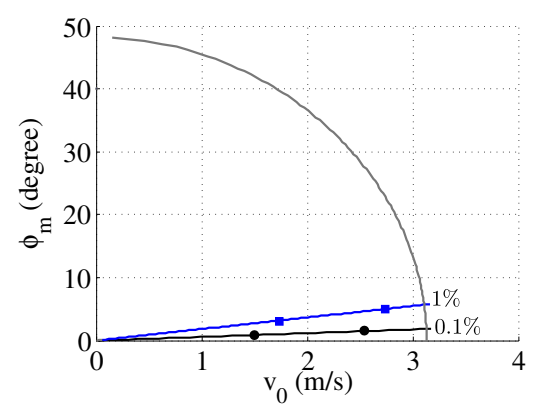

Figure 3.5: $v_{0} \approx v_{m}$ constraint lines for $1 \%$ and $0.1 \%$ difference between $v_{0}$ and $v_{m} . l=1 \mathrm{~m}$ and $\phi_{b}=0$

in Section 2.4). The actuation provided is not enough to sustain the gait. The walker keeps slowing down to lower and lower mid-stance speeds at each subsequent step till the losses equal the energy that the actuator can impart in one stance phase (the operating point moves to a regime 4 steady-state walk). To avoid such a situation, the actuation should be at least enough to attain a steady state walk by the next ground impact. In the limiting case, the actuation is just enough to maintain steady state (regime 4). Neglecting internal energy, the expression for $E_{\text {phase }}$ in Equation 3.11 reduces to the expression for $E_{\text {loss }}$ in Equation 2.14. Substituting Equation 2.14 into Case 4 in Section 2.4 yields

$$
v_{m}^{2}=\frac{4 g l \phi_{m} \phi_{c}}{\sin ^{2} 2 \phi_{m}}
$$

Plugging Equation 2.5 into the above equation gives the steady-state constraint line in terms of $v_{0}$ and $\phi_{m}$. Figure 3.4 plots the steady-state constraint lines for few different values of parameters. All operating points on the steady-state constraint line yield regime 4 walking. Only operating points on or below this line lead to steady-state walks (no slowing down).

\subsection{5 $v_{0} \approx v_{m}$ constraint}

For very small $\phi_{m}$ and reasonably high values of $v_{0}$, two undesirable effects are observed.

1. Since the variation in CoM height (Equation 1.7) is negligible, hardly any energy is transformed from potential to kinetic. The CoM velocity $v_{m}$ at the end of a stance phase is almost equal to the mid-stance CoM velocity $v_{0}$. This situation is like the case of the synthetic wheel (Section 1.4.3) but with very large internal energy requirement because of high step frequency.

2. Sub-phase and stance phase durations become very small and can be of the same 
order as the computation time step while solving for the dynamics of the walker. This can lead to appreciable errors in the computation of $v_{x, a v g}$ and $P_{\text {avg }}$.

Because of the impractically high internal energy requirement and for ease of computation, such operating points may be avoided. Accordingly, a $v_{0} \approx v_{m}$ constraint line is obtained after solving the dynamics of the walker for various operating points. Figure 3.5 shows these constraint lines for $1 \%$ and $0.1 \%$ difference between $v_{0}$ and $v_{m}$. They are superimposed on the take-off constraint line (no braking) for reference. Operating points above the $v_{0} \approx v_{m}$ constraint lines have a larger difference between $v_{0}$ and $v_{m}$ and are of interest for us.

\subsection{Optimal walker}

Once the limits on the choice of operating points are defined, the next logical step is to look for optimal operating points that prescribe the best way to walk. This requires choosing a suitable cost function and a method to compute and then minimize it. Section 1.2 justifies choosing mechanical power consumption as the cost function for engineering applications.

\subsubsection{Computation of average power and average velocity}

For an inverted pendulum walker using a compass gait with alternating legs, in addition to overcoming losses (Equation 2.14), some energy needs to be spent to move and place the finite mass swing-leg at every step $\left(E_{i n t}\right)$. At normal walking speeds, this energy might be much smaller than the losses. But at fast walking speeds, the contribution due to internal energy is likely to dominate. The swing leg can be modelled as a simple pendulum of mass $m_{l e g}$ and effective length $l_{s w}$. The internal energy is the energy required to drive this simple pendulum and can be derived from basic mechanics.

$$
E_{\text {int }}=8 m_{\text {leg }} l_{\text {sw }}^{2} \phi_{m}^{2} f^{2}
$$

where $f$ is the step frequency. For humans, the leg mass is about $10 \%$ of the body mass. The effective length of the swing leg can be taken as 0.5l. A higher duty factor implies lesser time available for driving the swing leg and hence higher $E_{\text {int }}$ than that in Equation 3.10. In the case of a rimless wheel, the distributed legs configuration reduces the internal energy requirement as there is no swing leg that needs to be driven and placed in time for the next step. $E_{\text {loss }}$ and $E_{\text {int }}$ need to be delivered continuously to the 
walker throughout a steady gait. Mechanical energy supplied to the walker in a stance phase is

$$
E_{\text {phase }}=E_{\text {loss }}+E_{\text {int }}
$$

Note that different muscles/motors could be used to supply the $E_{\text {loss }}$ and $E_{\text {int }}$ portions. The average mechanical power is given by

$$
P_{\text {avg }}=\frac{E_{\text {phase }}}{T}
$$

where the stance phase duration $(T)$ is computed by adding all the sub-phase durations $\left(T_{s p}\right.$; Equation 2.32) in the given regime. Taking $m, g, l, \phi_{a}$ and $\phi_{b}$ as fixed parameters, $P_{a v g}$ is a function of two variables $v_{0}$ and $\phi_{m}$. In abbreviated notation

$$
P_{\text {avg }}=f_{1}\left(v_{0}, \phi_{m}\right)
$$

Similarly, the average forward velocity is also a function of these two variables.

$$
v_{x, a v g}=\frac{2 l \sin \phi_{m}}{T}=f_{2}\left(v_{0}, \phi_{m}\right)
$$

Thus, $v_{0}$ and $\phi_{m}$ are the controllable variables that decide the average forward velocity and the average rate of mechanical work done for a steady walk. Both non-linear functions, $f_{1}$ and $f_{2}$, can be visualized in the form of contour plots for a given set of parameters (Figures 3.6a,b).

\subsubsection{Minimum mechanical power for inverted pendulum walker}

Any chosen operating point results in a particular value of average forward velocity $v_{x, a v g}$ and average mechanical power consumption $P_{\text {avg }}$. Figure 3.6 a shows the $v_{x, \text { avg }}$ contour plot for $m=65 \mathrm{~kg}, g=9.81 \mathrm{~m} / \mathrm{s}^{2}, l=1 \mathrm{~m}, \phi_{a}=10^{\circ}$ and $\phi_{b}=1^{\circ}$. Each contour represents all possible combinations of $v_{0}$ and $\phi_{m}$ that give the same value of $v_{x, a v g}$. Figure $3.6 \mathrm{~b}$ shows the contour plot for $P_{\text {avg }}$ for the same parameter values as above. Here, each contour represents combinations of $v_{0}$ and $\phi_{m}$ that give the same value of $P_{\text {avg. }}$. From the contour plots note that both $P_{\text {avg }}$ and $v_{x, \text { avg }}$ are a lot more sensitive to variation in $v_{0}$ than they are to variation in $\phi_{m}$. The envelope of the contour plots is bounded by the constraint lines discussed earlier.

A particular value of average forward velocity $v_{x, a v g}$ can be achieved by choosing any of several combinations of $v_{0}$ and $\phi_{m}$. One of these combinations results in the least mechanical power consumption and can be considered the optimum operating point for 


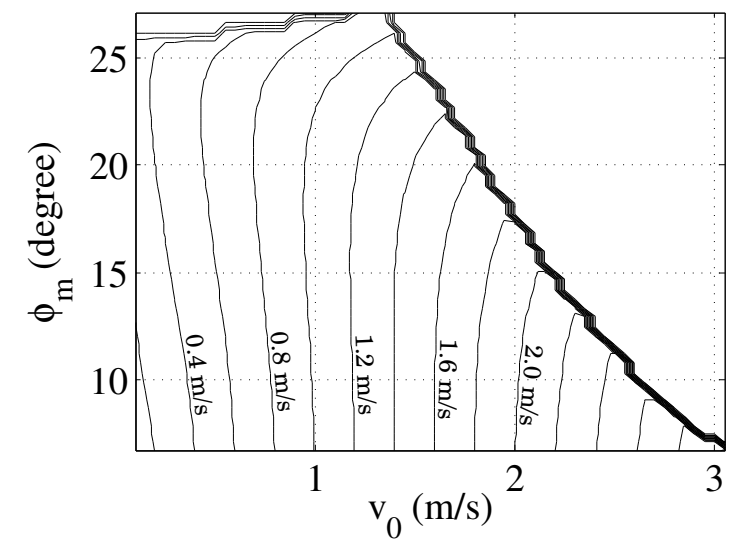

(a) $v_{x, a v g}$ contours. All operating points on a given contour lead to the same $v_{x, a v g}$.

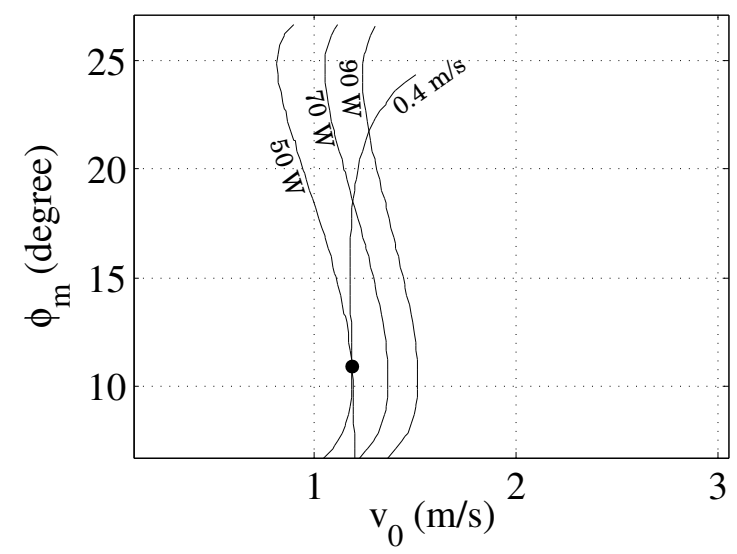

(c) Optimal operating point for a given average forward velocity.

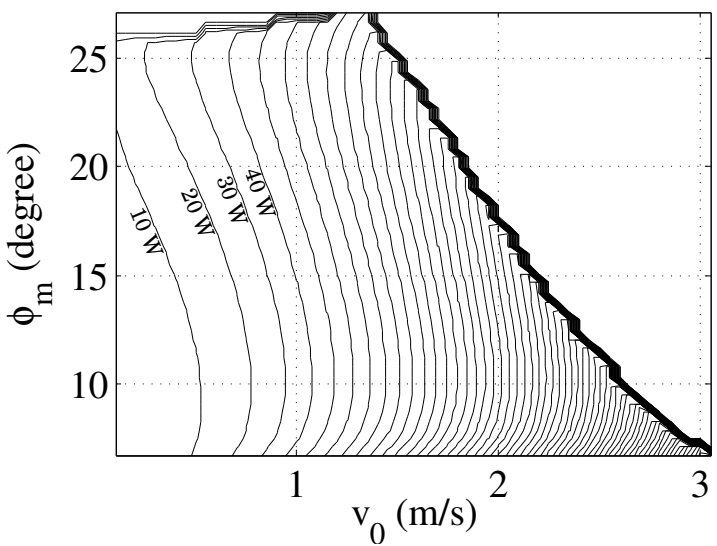

(b) $P_{\text {avg }}$ contours. All operating points on a given contour lead to the same $P_{\text {avg }}$.

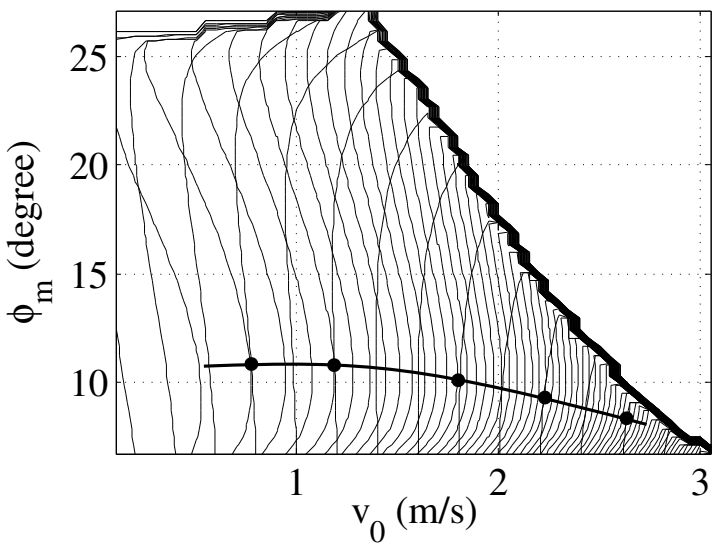

(d) Family of optimal operating points gives the optimal locus.

Figure 3.6: Average velocity and average power contours and construction of optimal locus. Parameters: $m=65 \mathrm{~kg}, l=1 \mathrm{~m}, \phi_{a}=10^{\circ}$ and $\phi_{b}=1^{\circ}$.

the given $v_{x, a v g}$. To find the optimal operating point, the lowest valued $P_{\text {avg }}$ contour the one that is tangential to the given $v_{x, a v g}$ contour - needs to be found. The point of tangency gives the optimal operating point (Figure 3.6c). Any other operating point on the given $v_{x, \text { avg }}$ contour has a higher mechanical power requirement.

Similarly, one can find the optimal operating points corresponding to increasing values of $v_{x, a v g}$ till one hits any of the constraint lines (Section 3.1). The family of optimal solutions thus obtained constitutes the optimal locus (Figure 3.6d). Choosing operating points on this locus ensures that the walker expends the minimum possible mechanical energy for all realizable speeds. 


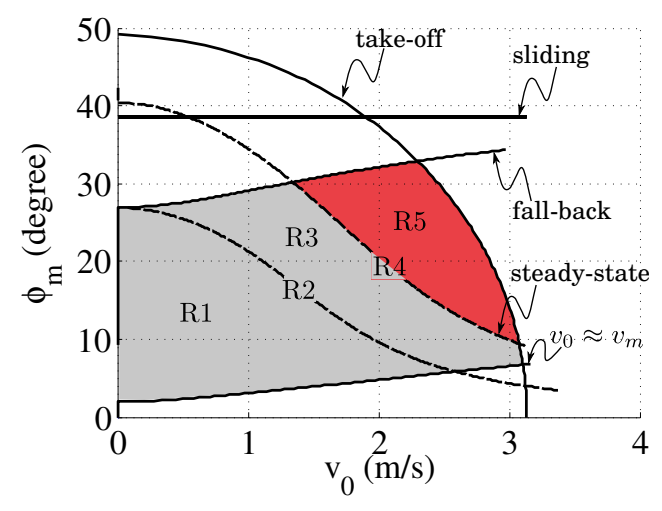

(a) $\phi_{a}=10^{\circ}$

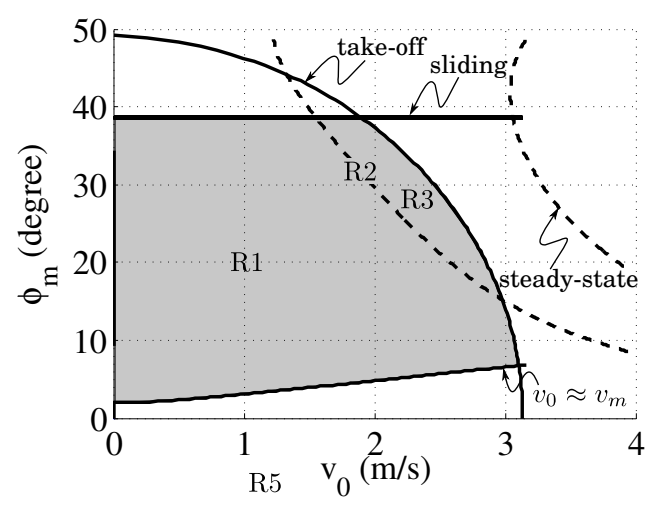

(b) $\phi_{a}=30^{\circ}$

Figure 3.7: Regions of operation for $l=1 \mathrm{~m}$ and $\phi_{b}=1^{\circ}$. R1-R5 correspond to regimes 1-5. The grey region indicates valid operating points that result in steady-state walking (regimes 1-4). The red region indicates operating points in regime 5 that result in a walk that keeps slowing down till the operating point reaches the regime 4 line. A higher actuator torque (b) results in a larger valid region of operation.

\section{$3.3 \quad$ Results}

\subsubsection{Regions of operation}

Figure 3.7a illustrates all the constraint lines (as derived in Section 3.1) for $l=1 \mathrm{~m}$, $\phi_{a}=10^{\circ}$ and $\phi_{b}=1^{\circ}$. Superimposing all the constraints, the valid region of operation for the inverted pendulum walker is obtained. Only operating points within this region are physically realizable.

Case 2 in Section 2.4 gives the regime 2 line (lower dashed line in Figure 3.7a). Operating points on this line correspond to regime 2 walking. It is the boundary between operating regions that yield regime 1 and regime 3 walks. Operating points on the steady state constraint line correspond to regime 4 walking (upper dashed line in Figure 3.7a). This line forms the boundary between regions of operating points that belong to regime 3 and regime 5. Regimes 1-4 give steady-state limit cycle walking.

The actuation torque plays a major role in defining the fall-back, steady-state and $v_{0} \approx v_{m}$ constraint lines and hence needs a closer look. Humans and several robots with finite-sized feet have actuated ankles. The vertical ground reaction force (GRF) acting on the foot of the trailing leg during the push-off (at double support) provides the actuation torque (see Figure 2.5). The point of application of this force is about a step length 
behind the stance foot thus providing a mechanical advantage.

$$
\tau_{a} \approx(\text { vertical GRF }) \times(\text { step length })
$$

The average value of the vertical GRF over the double support duration is a function of the duty factor $(\beta)$. A typical value is about $70 \%$ of the body weight i.e. $0.7 \mathrm{mg}$. Taking a typical step length $\left(l_{s}\right)$ of $0.7 \mathrm{~m}$, this amounts to a torque of about $0.5 \mathrm{mg}$ acting on the inverted pendulum. This implies $\phi_{a} \approx 30^{\circ}$ for humans (substitute $l=1 \mathrm{~m}$ and $\tau_{a}=0.5 \mathrm{mg}$ in Equation 2.16). For such a high value of $\phi_{a}$, the fall-back and steady-state constraint lines are pushed out beyond the take-off constraint line to regions of invalid operating points from a take-off perspective (Figure 3.7b). Hence, the valid region of operation is now bounded only by take-off, sliding and $v_{0} \approx v_{m}$ constraint lines. Only walking regimes 1, 2 and 3 are possible. For even higher actuation torques, only regime 1 walking is possible.

Humans, on account of having a relatively high actuator torque, have a larger valid region of operation (Figure $3.7 \mathrm{~b}$ ). Humans with emaciated calf muscles and robots with motors of low torque capability can have a smaller region of operation (Figure 3.7a).

With ankle actuation, going from regime 1 to higher regimes requires increasing the double support duration. This reduces the time available for the swing leg to be raised and placed for the next step. Hence, regimes 4 and 5 (continuous actuation) cannot be achieved since that would leave no time for driving the swing leg. Only operating points farther away from the steady state constraint line can be realized because of internal energy limitations. With hip actuation, it is possible to get continuous actuation albeit without the mechanical advantage.

\subsubsection{Optimal loci}

As the leg length is varied, the optimal loci (as derived in Section 3.2) obtained from the model are found to overlap when $v_{0}$ is normalized by $\sqrt{g l}$ (Figure 3.8a). For a broad range of actuation torques $\left(10^{\circ}<\phi_{a}<50^{\circ}\right)$, the shape and location of the optimal loci remains largely similar. With changing $\phi_{a}$, the loci shift up or down within a couple of degrees of each other. The optimal loci upto a non-dimensional speed of 0.7 have largely constant step angles. Thus choosing the right step angle and keeping it fixed over a broad range of speeds could lead to an inverted pendulum walker with minimum mechanical energy consumption. Chapter 4 describes the design, construction and working of Chatur - a fixed- $\phi_{m} 2 \mathrm{D}$ walker implemented using rimless wheels. 


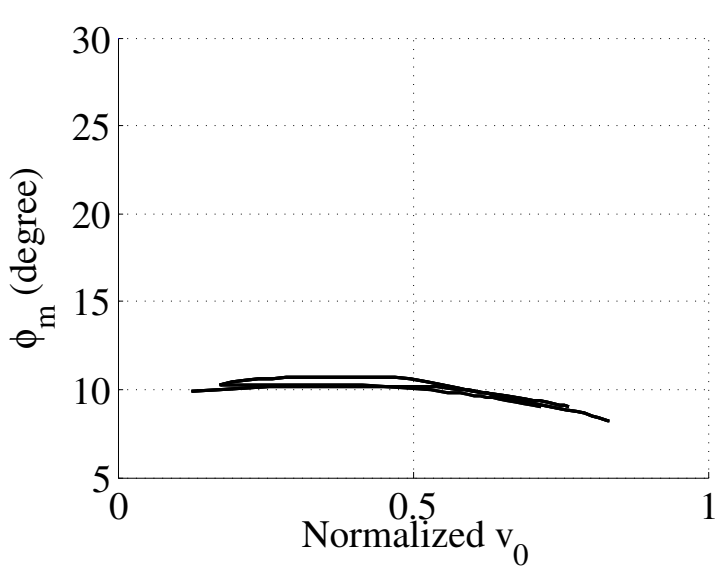

(a) Optimal loci for various $l$

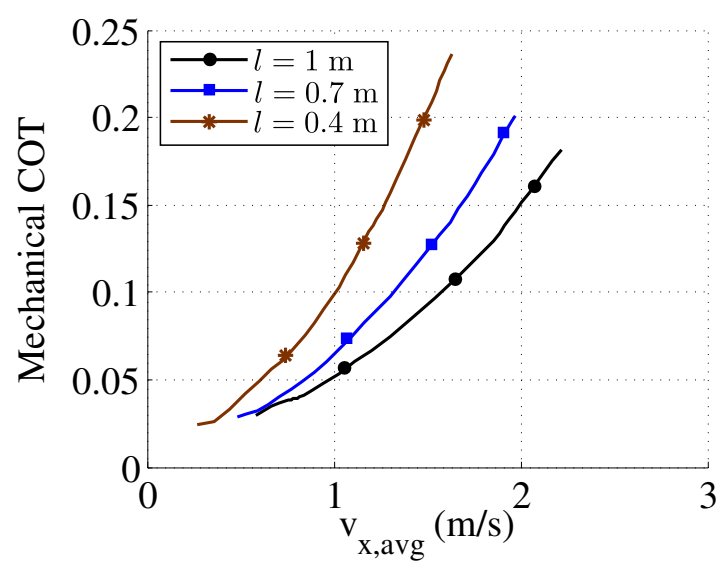

(c) Absolute $v_{x, a v g}$ for various $l$

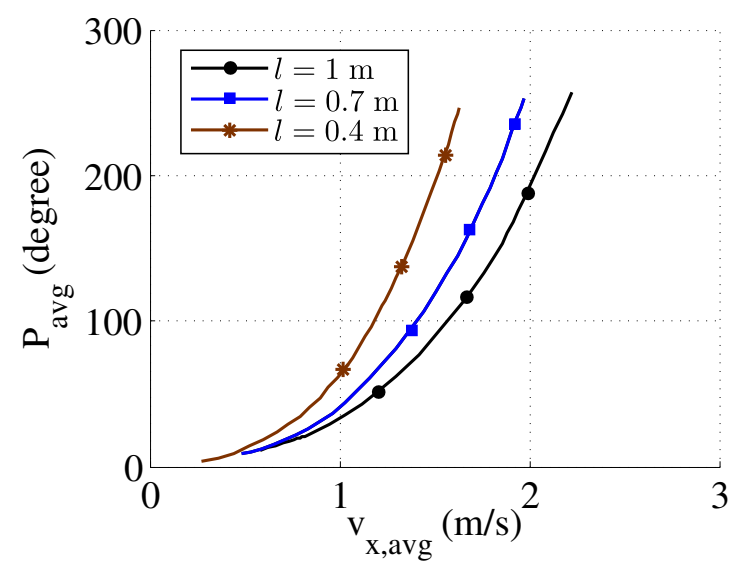

(b) $P_{\text {avg }}$ versus $v_{x, a v g}$ for various $l$

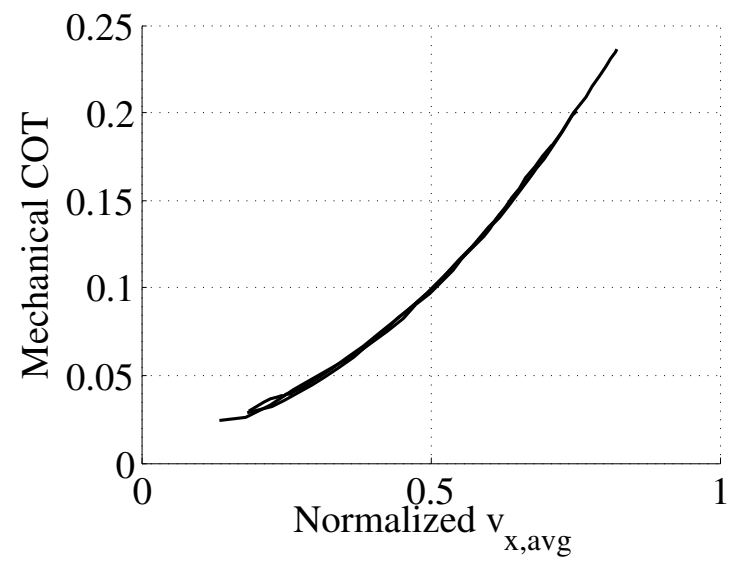

(d) Normalized $v_{x, a v g}$ for various $l$

Figure 3.8: Effect of changing $l$ on optimal locus and the corresponding $P_{\text {avg }}$ and mechanical cost of transport. Parameters: $m=65 \mathrm{~kg}, g=9.81 \mathrm{~m} / \mathrm{s}^{2}, \phi_{a}=10^{\circ}$ and $\phi_{b}=1^{\circ}$.

Figure $3.8 \mathrm{~b}$ plots the $P_{\text {avg }}$ versus $v_{x, a v g}$ assuming the walker always chooses operating points on the optimal locus. Figure 3.8c plots the corresponding mechanical cost of transport (Equation 1.4) for this optimal inverted pendulum walker. Larger leg lengths lead to smaller mechanical CoT for any given speed. Dividing $v_{x, a v g}$ by $\sqrt{g l}$, the nondimensional average forward velocity is obtained. Plotting the mechanical CoT versus this normalized $v_{x, a v g}$ (Figure 3.8d), almost overlapping curves are obtained irrespective of leg lengths.

Smaller step angles lead to lesser impact losses. But they warrant larger step frequencies that increases the internal energy requirements. Conversely, larger step angles lead to lower $E_{\text {int }}$ but higher $E_{\text {loss }}$. The shape and location of the optimal locus in the $v_{0}-\phi_{m}$ plane is a result of the trade-off between $E_{\text {loss }}$ and $E_{\text {int }}$. Hence, the accuracy of the models for losses (Equation 2.14) and swing leg actuation (Equation 3.10) play a key role 
in defining the optimal locus correctly. The internal energy requirement changes when taking into account the fact that the time available for the swing leg to be driven goes on reducing as one goes from regime 1 to regime 4. Also if the configuration of the inverted pendulum walker is a rimless wheel instead of a compass, the internal energy required is much smaller as there is no swing leg to actuate. Springy legs reduce impact losses and shift the optimal locus upwards. Lighter legs reduce the internal energy requirement thus shifting the optimal locus downwards.

The inverted pendulum walker modelled here is quite lossy and hence has relatively higher mechanical CoT compared to human walking. Humans store part of the impact energy in elastic tendons on the front side of the shin. For a man-made inverted pendulum walker, adding compliant elements to store and release the impact energy reduces the mechanical power requirement. This shifts the optimal locus upwards to larger step angles as is typically observed in humans $\left(15^{\circ}-25^{\circ}\right)$. However, it should be noted that human walking gait optimizes for metabolic energy and not mechanical energy. Hence, even with reduced impact losses, the inverted pendulum walker can have a different optimal locus compared to human walking. Experimental measurements (Cavagna et al., 2000) show that the step lengths (and hence the step angles) for humans increases from $0.5 \mathrm{~m}$ to $0.9 \mathrm{~m}$ with walking speed increasing from $0.5 \mathrm{~m} / \mathrm{s}$ to $2 \mathrm{~m} / \mathrm{s}$.

\subsubsection{Discussion}

The constraint lines and optimal loci on the $v_{0}-\phi_{m}$ plane can be easily mapped to the $v_{x, a v g}-l_{s}$ and $f-l_{s}$ planes by using known relations between the quantities. The operating point of the walker can also be specified by the combination of steady state mid-stance velocity $\left(v_{0 s s}\right)$ and step angle $\left(\phi_{m}\right)$. $v_{0 s s}$ can be lower than $v_{0}$ if the actuation torque is too low. Assuming that the actuation torque is high enough to achieve steady state within the second ground impact, the $v_{0 s s}$ is either equal to or uniquely related to $v_{0}$.

\subsection{Conclusion}

The operating point of the inverted pendulum walker is specified by a combination of initial mid-stance velocity and step angle chosen for a given walk. A framework of physical constraints that limit the choice of operating points is proposed. Five physical constraints that delineate the valid region of operation are presented. These constraints impose 
fundamental limits on the walking. For any given speed, the optimal operating point that ensures minimum mechanical energy expenditure, based on tangency of the power and velocity contours, is proposed. Applying the proposed method to different speeds, the locus of optimal operating points is obtained. The shape and location of this optimal locus is sensitive to the models chosen for loss and internal energy. Using an appropriate constant step angle over a broad range of speeds could lead to an inverted pendulum walker that is close to optimal. This optimality is different from the optimality based on minimum metabolic cost. 


\section{Chapter 4}

\section{Hardware Design and Control Topology}

This chapter describes the hardware design and proposes a control topology for pulsed torque actuation of a dual-rimless-wheel based 2D walker named Chatur. A characteristic feature of this class of mechanical loads is that the reflected torque has a cyclic variation that repeats several times within a mechanical revolution. The torque pulse tries to put in just the right amount of energy to maintain the forward motion of the mobile platform. Continuous actuation with constant torque is a special case where the duty ratio of the pulse is $100 \%$. Section 4.1 details the design and construction of the complete hardware set-up of Chatur. Section 4.2 describes the design and implementation of the proposed control scheme for pulsed torque actuation of Chatur ensuring synchronization of the rimless wheels. The BLDC motor drive uses non-commutating current feedback to reduce current spikes during sector transitions. Section 4.3 shows experimental waveforms that validate the working of the proposed controller.

\subsection{Hardware design}

Chatur is a fixed step-angle dynamic walker implemented using the rimless wheel approach. The CoM kinematics and dynamics of a rimless wheel with zero radius of gyration is similar to that of an inverted pendulum walker. In both cases, the CoM traces a trajectory consisting of a series of arcs (shown in dashed lines in Figure 1.6a,b). The spoke length of the rimless wheel is equivalent to the leg length of the biped. The inter- 
spoke angle is equivalent to the inter-leg angle of the compass walker at foot-strike and is twice the step angle (Figure 1.6b). The distance between the far ends of two consecutive spokes signifies a side of the rolling polygon and stands for the step length of the biped. Since the spokes are spaced at constant angles, this rimless wheel based walker cannot vary its step angle unlike the more dextrous biped using alternating legs.

Each time a new leg is to be placed on the ground, the rimless wheel makes it easily possible because of its distributed legs configuration. This avoids the need to drive a swing leg fast enough to be placed in time for the next step. Hence, the rimless wheel approach leads to a lower internal energy requirement compared to a compass gait with alternating legs.

A single rolling rimless wheel can fall sideways. To ensure lateral stability two contralateral rimless wheels are used (see Figure 4.2) each driven by a separate BLDC hub motor. It is effectively a walker with an actuated hip joint. There is no mechanical advantage and hence the motor torque requirement is higher compared to foot actuation. In order to ensure fidelity with the 2D inverted pendulum model, both the rimless wheels need to move in synchronism. The foot point of each leg of a rimless wheel should hit the ground exactly as its counterpart on the other wheel. By synchronizing the two wheels, motion is limited to the sagittal plane making it a 2D walker. Actuation can be either pulsed or continuous by energizing the motor accordingly.

\subsubsection{Mechanical subsystem}

The mechanical subsystem comprises the two rimless wheels, a welded mild steel (MS) frame and enclosures for the onboard electronics. No attempt is made to reduce weight of the system, so that the heavy parts, such as MS frame $(7 \mathrm{~kg})$ and sealed lead acid (SLA) batteries $(15 \mathrm{~kg})$, can emulate some payload weight. All the CAD drawings/renderings are given in Appendix B (Section B.1).

\section{Rimless wheels}

Figure 4.1 shows the construction of a rimless wheel with a BLDC hub motor as the actuator (Figure 4.1a). The reasons for choosing a hub motor are threefold:

1. The bearings are designed to take lateral shocks as the target application of these motors are electric scooters and electric assist bicycles. 


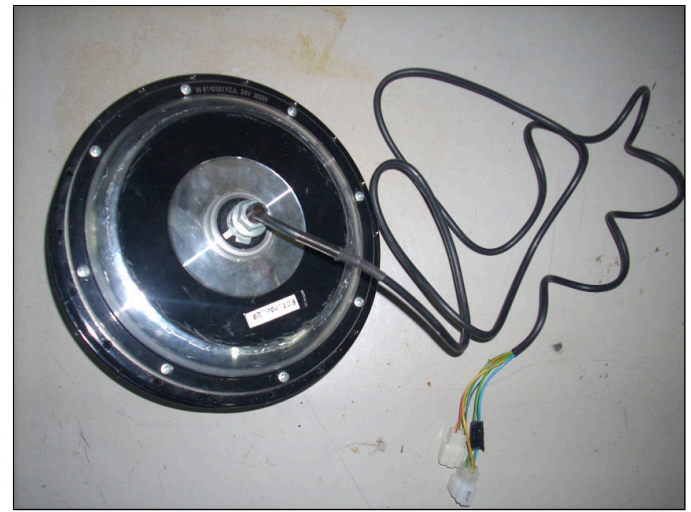

(a) Brushless dc hub motor

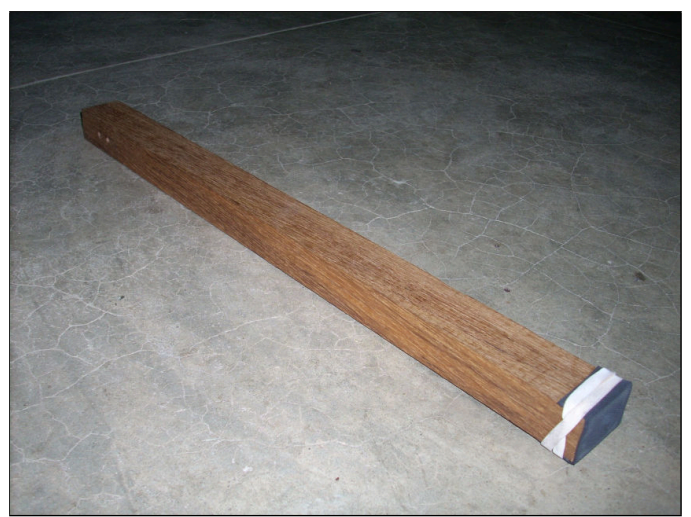

(c) Teak wood spoke with rubber 'foot'

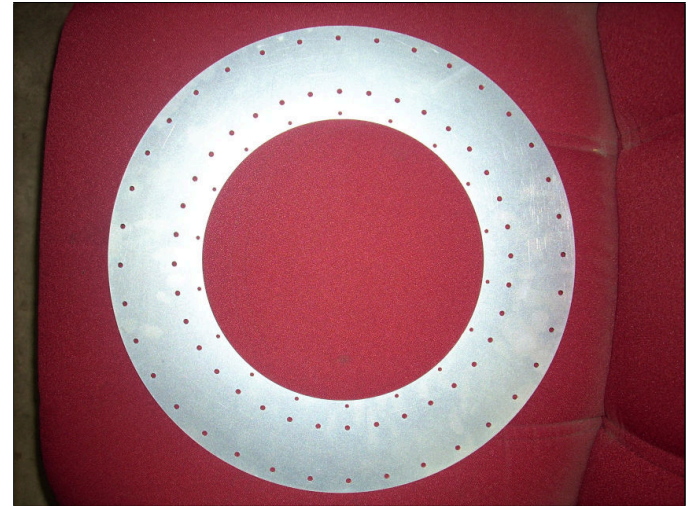

(b) Galvanized MS annular disc

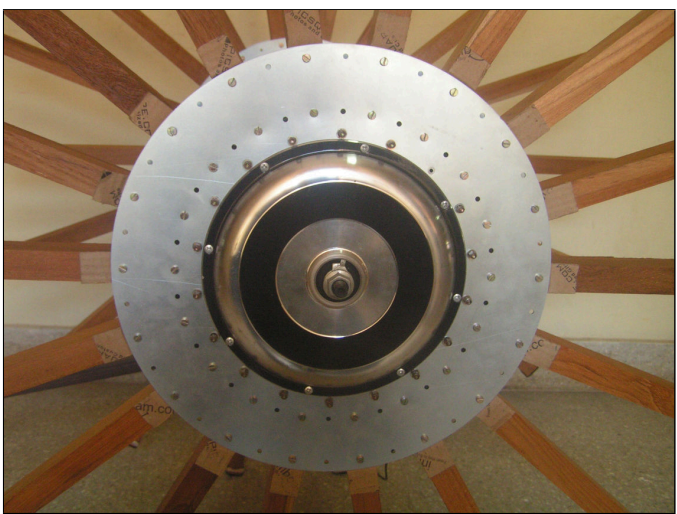

(d) Assembly of all three parts

Figure 4.1: Construction of a rimless wheel.

2. The outer rotor allows for easy construction of the wheel without the need for a transmission. The spokes can be directly attached to the outer rotor.

3. These motors are capable of low speed, high torque operation.

Eighteen equidistant spokes at $20^{\circ}$ intervals are attached to the outer rotor of the hub motor. Initially, aluminium spokes were explored. But owing to unavailability of aluminum extrusions of required dimensions, wooden spokes are used $(457 \mathrm{~mm} \times 33 \mathrm{~mm}$ $\times 25 \mathrm{~mm}$, Figure 4.1c). The spokes are attached to the rotor via mild steel annular discs using M4 nuts and bolts (Figure 4.1d). The annular discs are fabricated using laser cutting (Figure 4.1b; CAD drawing Figure B.1). Rivet nuts, as opposed to normal hex nuts, are used to reduce the stress on the bolt threads. MS washers are used to prevent brinelling of the annular disc. Spring washers are used to prevent the loosening of the joints due to vibrations on impacts. The distal ends of the distributed legs (spokes) have rubber 'feet' in order to reduce impact vibrations and losses. After assembly, each rimless 

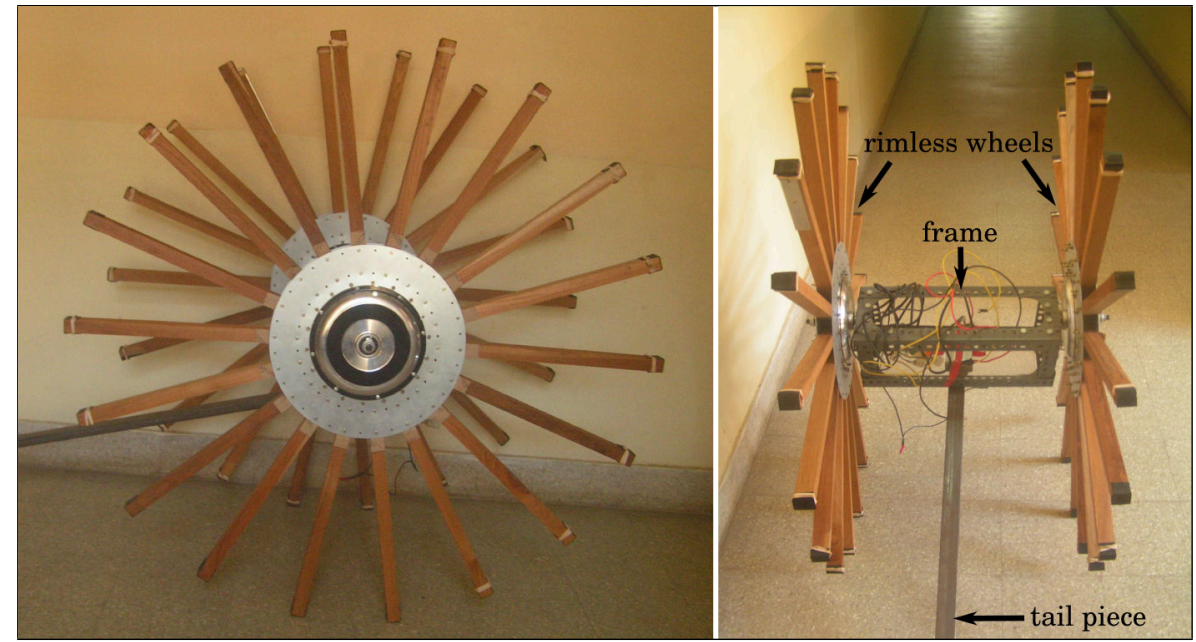

(a) Version-1: slotted angle frame.

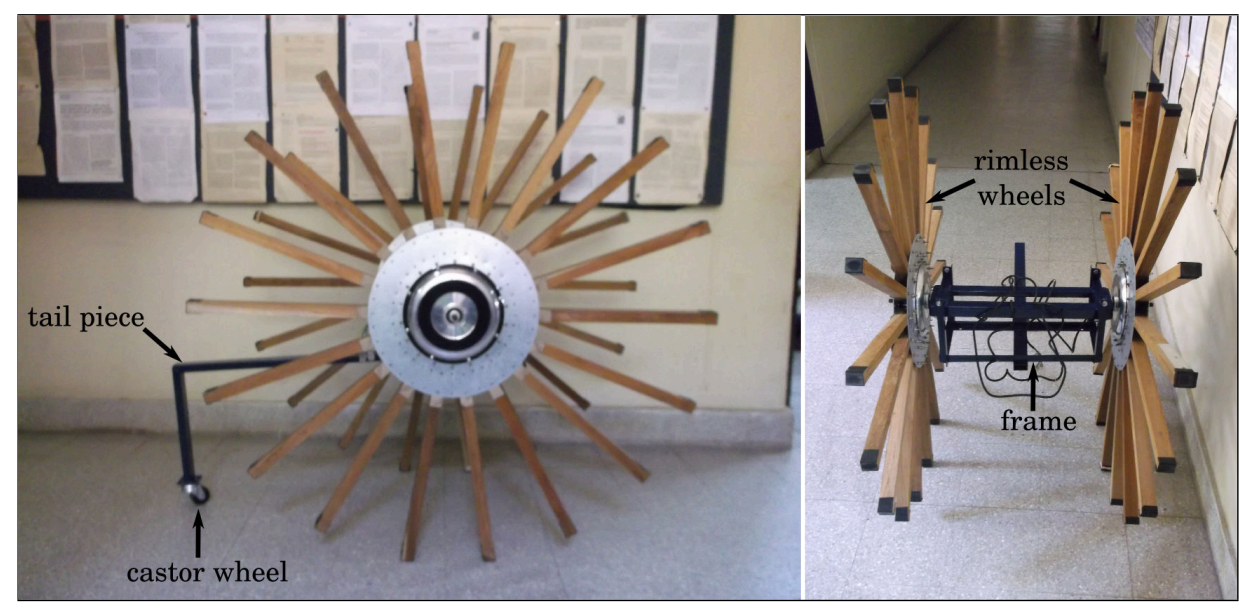

(b) Version-2: welded mild steel frame.

Figure 4.2: Full mechanical assembly of Chatur excluding enclosures for onboard electronics: two contralateral rimless wheels and frame with attached tail piece.

wheel weighs $12 \mathrm{~kg}$.

\section{Frame}

The first version of the frame (chassis) was built using mild steel slotted angles (Figure 4.2a). Availability of several slots providing a flexibility in modifying the frame size was the chief reason for choosing slotted angles. Joints between slotted angle members were made using corner plates and M7 nuts/bolts.

When energized, the motors try to rotate the wheels and a counter torque acts on the 
stators which are attached to the chassis. To prevent this counter torque from rotating the frame rather than the wheels, a tail piece is introduced (Figure 4.2). The bottom end of the tail has a small castor wheel resting on the floor. The ground reaction force on the castor helps resist the counter torque on the frame. An alternate design without the tail piece is possible by using a counterweight (located away from the axle) on the frame (Robotics-Unlimited, 2014). The top plate of the castor wheel needs to be horizontal in order to avoid undesirable yawing torques that try to turn Chatur to the left or right. Initially, a swivelling castor wheel was used. But any lateral component of the ground reaction force on the castor wheel resulted in appreciable yawing torque on Chatur. Hence, it is replaced with a normal non-swivelling castor wheel.

The first version of the frame had the following problems:

1. Wheel alignment: the planes of the rimless wheels were not parallel (they had some camber and toe-in) resulting in a large reflected torque on the motor.

2. Wobbling wheels: lack of stiffness in the slotted angle members resulted in a flimsy frame. The planes of the rimless wheels kept wobbling leading to dynamics very different from that of a $2 \mathrm{D}$ rimless wheel.

3. Assembly issues: attaching the rimless wheel to the frame was cumbersome because of inaccessibility of the nuts on the inner side.

In order to overcome the above short-comings, a second version of the frame is designed and constructed using precision welded mild steel angles (Figure 4.2b). This frame consists of three parts: (i) axle or main frame, (ii) battery carrier and (iii) tail piece and castor wheel assembly.

Figure B.2 shows the CAD drawing of the main frame. It is made of $35 \mathrm{~mm}$ L-angles of $5 \mathrm{~mm}$ thickness. The two motors are attached to this part by placing the stator axle of the hub motor in the $11 \mathrm{~mm}$ slots provided and then fastening using nuts. Figures B.3-B.5 show the CAD drawings of the battery carrier (along with the main frame). It is made of $20 \mathrm{~mm}$ L-angles of $3 \mathrm{~mm}$ thickness. Figure B.6 shows the CAD drawing of the tail piece. It is made of rectangular MS pipe (40 $\mathrm{mm} \times 20 \mathrm{~mm} ; 2 \mathrm{~mm}$ thickness). Figure B.7 shows the CAD drawing of the full assembly of the frame. 


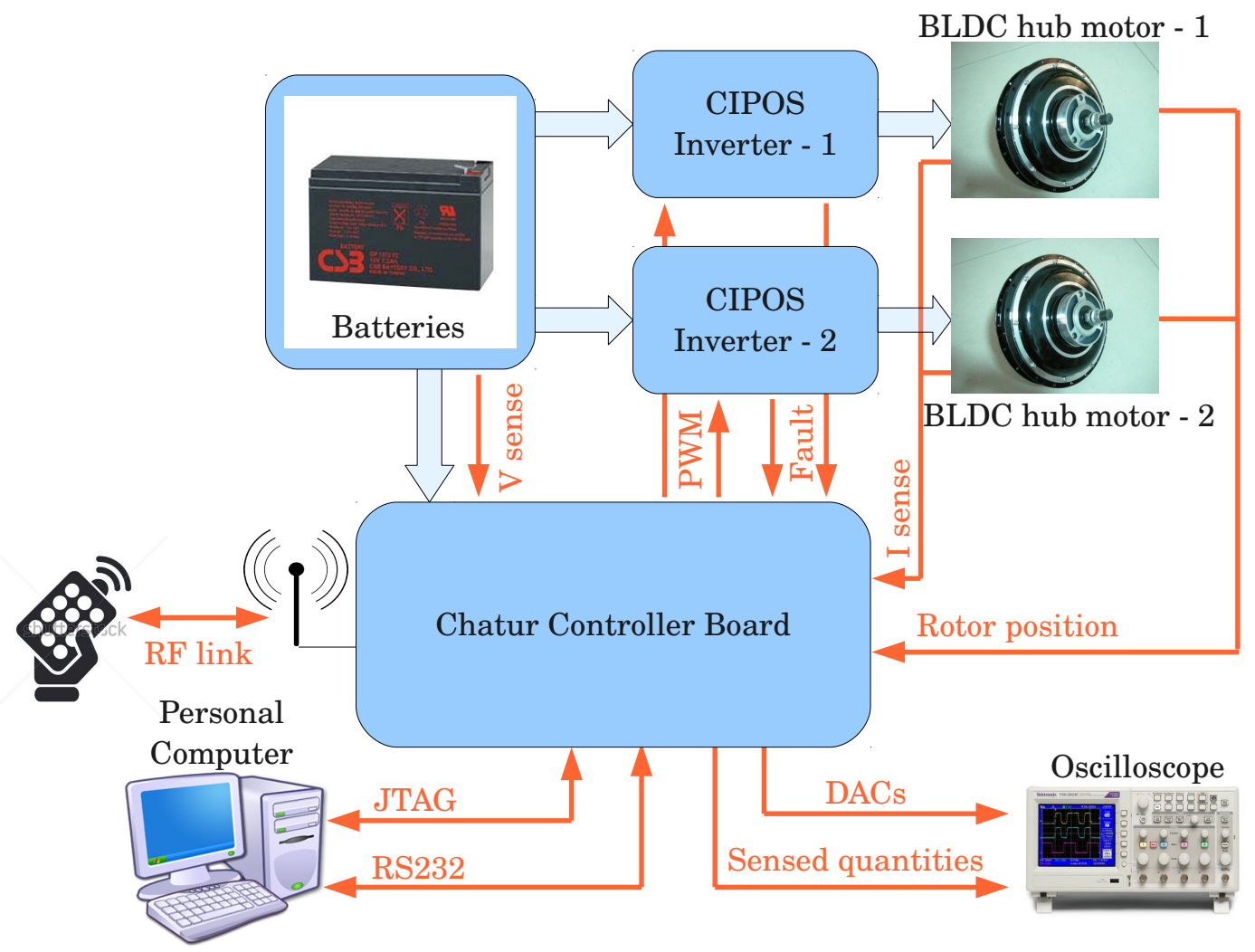

Figure 4.3: Block diagram of electrical subsystem.

\section{Enclosures}

An aluminium enclosure (Figure B.8) is designed for housing the power and control electronics. A polycarbonate enclosure (Figure B.9) is designed for housing the terminal block where connections are made between the batteries, motors, manual switches, fuses and heat-sink fans. Both the enclosures along with the electronics sit on top of the main frame.

\subsubsection{Electrical subsystem}

The block diagram of the complete electrical subsystem - energy source, actuators and electronics - is shown in Figure 4.3. 
Table 4.1: Specifications of sealed lead acid batteries (CSB GP1272).

\begin{tabular}{lc}
\hline Parameter & Value \\
\hline Voltage & $12 \mathrm{~V}$ \\
Ampere-hour & $7.2 \mathrm{Ah}$ \\
Mass & $2.4 \mathrm{~kg}$ \\
Dimensions & $150 \mathrm{~mm} \times 95 \mathrm{~mm} \times 65 \mathrm{~mm}$ \\
\hline
\end{tabular}

\section{Energy source}

The energy source is a set of SLA batteries (specifications in Table 4.1). Two batteries in series give $24 \mathrm{~V}$ nominally. Two such pairs provide the dc links for the inverters that drive the two motors. One more pair is used to power the control electronics. Thus, six batteries are used in total. Fuse protection (20 A, 20 A, 0.8 A) is provided for each of the pairs.

\section{Actuators}

Each of the two wheels is actuated by a three-phase BLDC hub motor (specifications in Table 4.2). The torque constant $\left(K_{t}\right)$ and winding inductance $\left(L_{a}=L_{b}=L_{c}=L_{p h}\right)$ and resistance $\left(R_{a}=R_{b}=R_{c}=R_{p h}\right)$ are determined experimentally by conducting blocked rotor tests. Figure 4.4 shows the set up for measuring the stalling torque using a digital weighing scale and known lever arm. The measurement of torque constant has a $10 \%$ tolerance owing to the torque variation in the motor with change in rotor position.

A hall effect position sensor built into the motor gives the rotor position as a 3-bit digital output. Within each electrical cycle, the sensor gives six different position outputs or sector numbers. For the 25 pole pair motor, this translates to a mechanical position resolution of $2.4^{\circ}$. The sequence of sector numbers is 5-4-6-2-3-1 for counter-clockwise rotation (CCW; Table 4.3) and is used for the right motor on Chatur. The reverse sequence gives clockwise $(\mathrm{CW})$ rotation and is used for the left motor. At any instant, only two of the three phase windings of the BLDC motor carry current while the third one is left unused. This leads to six possibilities as shown in Figure 4.5. Each of the six current directions leads to a different stator mmf space vector orientation which the permanent magnet rotor tries to catch up to (Figure 4.6b). Table 4.3 shows the winding current commutation sequence for CCW rotation. CW rotation is achieved by reversing 


\begin{tabular}{lc}
\hline Parameter & Value \\
\hline Power & $300 \mathrm{~W}$ \\
Voltage & $24 \mathrm{~V}$ \\
Current & $12 \mathrm{~A}$ \\
Pole pairs & 25 \\
Torque constant $\left(K_{t}\right)$ & $0.6 \mathrm{Nm} / \mathrm{A}$ \\
Phase resistance $\left(R_{p h}\right)$ & $0.35 \Omega$ \\
Phase inductance $\left(L_{p h}\right)$ & $0.12 \mathrm{mH}$ \\
Mass & $5.2 \mathrm{~kg}$ \\
\hline
\end{tabular}

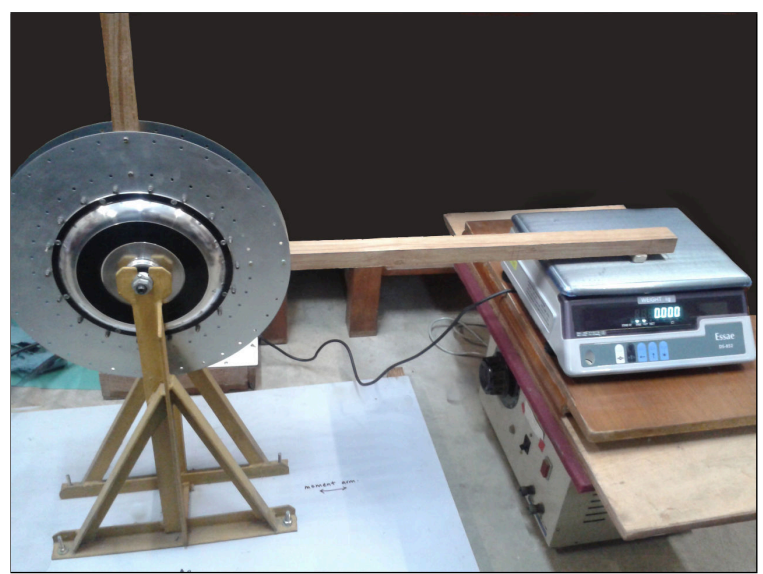

Table 4.2: Specifications of BLDC motor. Figure 4.4: Block rotor test of BLDC motor.

\begin{tabular}{ccccc}
\hline Sector no. & Motor current & \multicolumn{4}{c}{ Inverter state } \\
& direction & A & B & C \\
\hline$(101)_{2}=5$ & $\mathrm{~A}^{+} \mathrm{B}^{-}$ & sw & 0 & $\mathrm{x}$ \\
$(100)_{2}=4$ & $\mathrm{~A}^{+} \mathrm{C}^{-}$ & sw & x & 0 \\
$(110)_{2}=6$ & $\mathrm{~B}^{+} \mathrm{C}^{-}$ & $\mathrm{x}$ & $\mathrm{sw}$ & 0 \\
$(010)_{2}=2$ & $\mathrm{~B}^{+} \mathrm{A}^{-}$ & 0 & $\mathrm{sw}$ & $\mathrm{x}$ \\
$(011)_{2}=3$ & $\mathrm{C}^{+} \mathrm{A}^{-}$ & 0 & $\mathrm{x}$ & $\mathrm{sw}$ \\
$(001)_{2}=1$ & $\mathrm{C}^{+} \mathrm{B}^{-}$ & $\mathrm{x}$ & 0 & $\mathrm{sw}$ \\
\hline
\end{tabular}

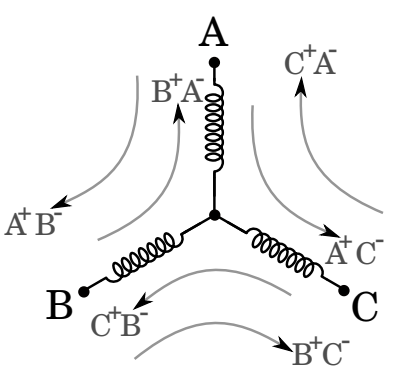

Table 4.3: Commutation sequence (CCW rotation) of BLDC motor. $\mathrm{sw}=$ switching, $0=$ clamped to $0, \mathrm{x}=$ both switches $\mathrm{OFF}$.

Figure 4.5: Current directions of BLDC motor

the current direction for each sector.

\section{Electronics}

The power electronics circuitry consists of two CIPOS $^{\mathrm{TM} *}$ based three-phase inverters to drive the two BLDC motors. The signal electronics circuitry comprises sensing, signal conditioning and control circuits that reside in a $\mathrm{PSoC}{ }^{\circledR} 5^{\dagger}$ based printed circuit board (PCB) called Chatur controller board. The complete circuit schematics for both the boards are given in Appendix B (Section B.2).

\footnotetext{
*Control Integrated POwer System from Infineon

${ }^{\dagger}$ Programmable System on Chip from Cypress Semiconductor
} 


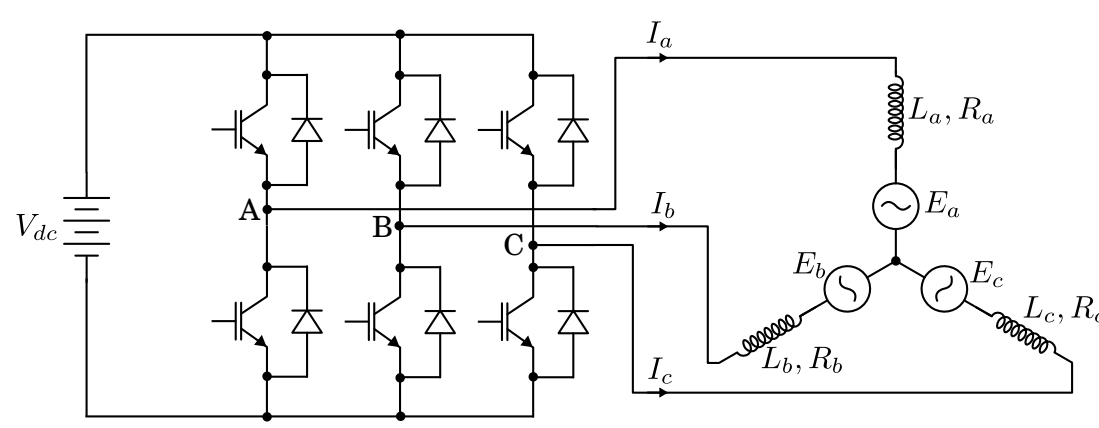

(a)

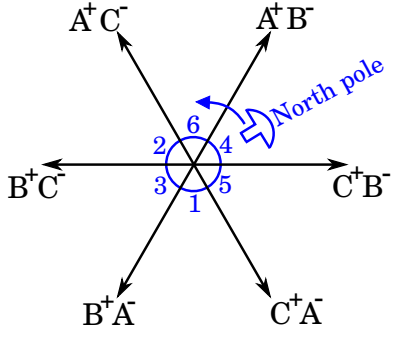

(b)

Figure 4.6: (a) Circuit schematic of three phase inverter and BLDC motor. (b) Stator mmf orientations and rotor positions over one electrical cycle.

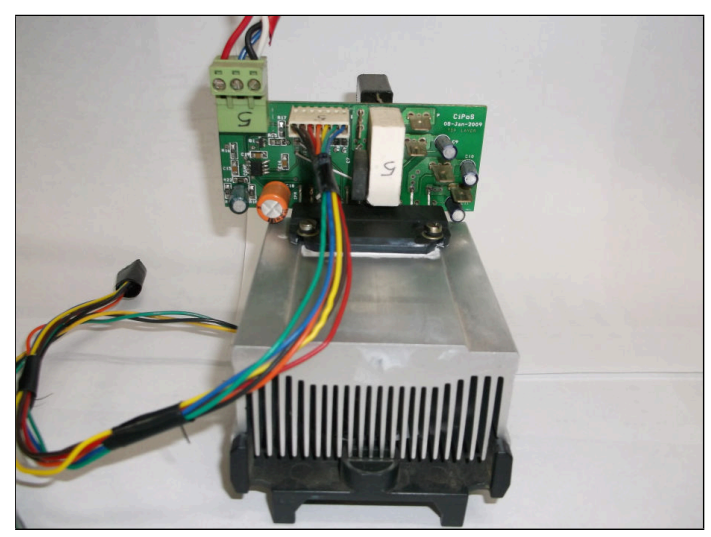

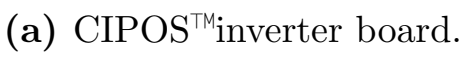

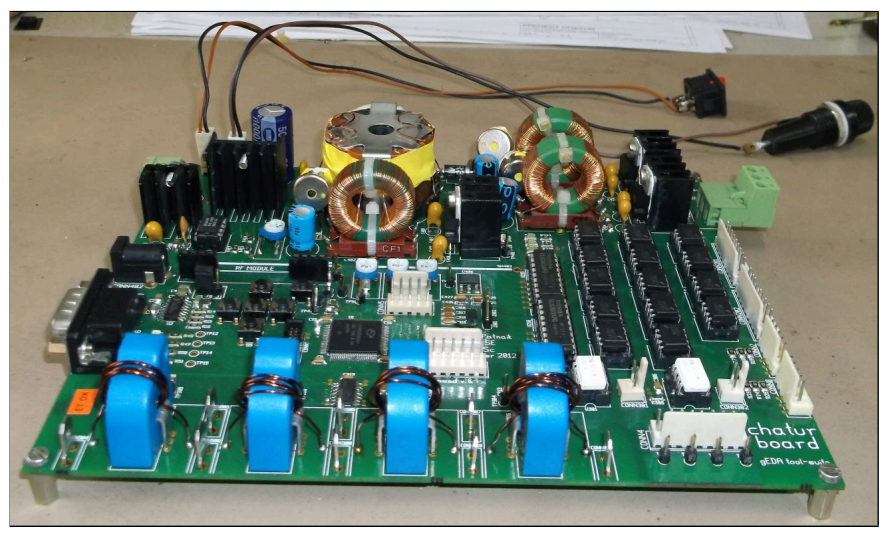

(b) Chatur controller board.

Figure 4.7: PCBs designed for Chatur: power and control electronics

CIPOS $^{\mathrm{TM}}$ inverter board: This board ${ }^{\ddagger}$ (Figure $4.7 \mathrm{a}$ ) is based on the reference design given in Infineon's application note (JunBae Lee and Chung, 2010). The specifications of the board are listed in Table 4.4 and its circuit schematic is given in Figure B.10. It uses the CIPOS ${ }^{T M}$ Integrated Power Module (IKCS22F60AA). This module is a conventional three-phase inverter along with gate drive circuit for all six Insulated Gate Bipolar Transistors (IGBTs) in one package. The board has a comparator based circuit for generating a fault signal in case of over-current or over-temperature. The current trip value is set at $15 \mathrm{~A}$. The inverter is operated at a switching frequency of $16.33 \mathrm{kHz}$ which yields a PWM cycle time of $61.2 \mu \mathrm{s}$ (less than one-fifth of the motor winding time constant, $\left.L_{p h} / R_{p h} \approx 350 \mu \mathrm{s}\right)$. To prevent shorting of the dc link, a dead time $(3 \mu \mathrm{s})$ is provided between the turn-OFF of a switch and the turn-ON of its complementary switch on the same inverter leg. Cooling for CIPOS ${ }^{T M}$ is provided by an off-the-shelf force-cooled heatsink (with attached fan) typically used for any Socket-478 CPU (Pentium-4/Celeron).

\footnotetext{
‡Designed during the author’s M.Tech project at IISc, Bangalore (Patnaik, 2009, chap. 13)
} 
Table 4.4: CIPOS $^{\top M}$ inverter board specifications.

\begin{tabular}{ll}
\hline Inputs & (1) $24 \mathrm{~V}$ dc link, $12 \mathrm{~A}$ \\
& (2) Power supply for gate drive $(15 \mathrm{~V})$ \\
& $\begin{array}{l}\text { and fault detection circuit }(5 \mathrm{~V}) \\
\text { (3) Six PWM control signals for the IGBTs }\end{array}$ \\
\hline Outputs & (1) Three pole voltages of the inverter \\
& (2) Fault indication to controller \\
\hline Dimensions & $87 \mathrm{~mm} \times 37 \mathrm{~mm} \times 1.6 \mathrm{~mm}$ \\
\hline
\end{tabular}

The circuit schematic in Figure 4.6a shows the connection of the inverter and BLDC motor. $V_{d c}$ denotes the $24 \mathrm{~V}$ dc link coming from battery. The reference directions of the phase currents $\left(I_{a}, I_{b}\right.$ and $\left.I_{c}\right)$ are indicated. The winding back EMFs $\left(E_{a}, E_{b}\right.$ and $\left.E_{c}\right)$ can be neglected $(<1 \mathrm{~V})$ for the low speed operation $(<10 \mathrm{rpm})$ in the present application. For CCW rotation of motor, the inverter switching states in the six sectors are indicated in Table 4.3. 'sw' indicates that the corresponding inverter leg is switching between $V_{d c}$ and $0 \mathrm{~V}$ with a duty ratio set by the torque controller (see Section 4.2.1). ' 0 ' indicates that the inverter leg is clamped to $0 \mathrm{~V}$ i.e the bottom device is $\mathrm{ON}$ and the top device is OFF. ' $x$ ' indicates that both the switches in the inverter leg are OFF.

Chatur controller board: This board is a custom PCB designed for controlling Chatur (Figure 4.7b). The design is carried out using the open source gEDA tool suite. The specifications of the board are listed in Table 4.5. The top-level block diagram and the circuit schematics of the board are given in Figures B.11-B.15. This board houses the $\mathrm{PSoC}^{\circledR} 5$ microcontroller (CY8C5568) and its peripheral circuits in addition to the circuits for housekeeping power supply, sensing, signal conditioning, communication, opto-isolation, and other miscellaneous tasks.

Sensors for motor currents, battery voltages, rotor-position and an accelerometer are provided. Four hall effect current sensors (LEM LTS25NP) are used for sensing phase currents of both motors $\left(I_{a 1}, I_{b 1}, I_{a 2}\right.$ and $\left.I_{b 2}\right)$. Each current carrying wire makes six turns around the current sensors giving a sensitivity of $150 \mathrm{mV} / \mathrm{A}$. The third phase current $\left(I_{c 1}\right.$ and $\left.I_{c 2}\right)$ is left unmeasured as it can be derived from the other two phase currents. Provision for sensing battery voltages - using resistive divider followed by isolation amplifier — is available on the board but is left unused. Since the built-in position sensors of the BLDC motor are of open-collector type, they need pull-up resistors that 
Table 4.5: Chatur controller board specifications.

\begin{tabular}{ll}
\hline Inputs & (1) Power \\
& (a) $24 \mathrm{~V}, 0.6$ A power supply (for flyback) \\
& (b) $5 \mathrm{~V}$ aux. power supply (unused in present work) \\
& (2) Sensing \\
& (a) Motor currents $\left(I_{a 1}, I_{b 1}, I_{a 2}\right.$ and $\left.I_{b 2}\right)$ \\
& (b) Battery voltages $\left(V_{d c 1}\right.$ and $\left.V_{d c 2}\right)$ \\
& (c) Rotor positions $(3$-bit digital output from each motor) \\
& (3) Others \\
& (a) JTAG programming and debugging interface \\
& (b) Remote control signal from RF transmitter \\
& (c) Fault indication from inverters \\
\hline Outputs & Six PWM control signals for each inverter \\
\hline Dimensions & 190.5 mm $\times 146.9$ mm $\times 1.6$ mm \\
\hline
\end{tabular}

are provided on the Chatur board. A MEMS accelerometer (MMA7331) is used to detect the ground impacts. When used in the $4 \mathrm{~g}$ sensitivity range, it gives $363 \mathrm{mV} / \mathrm{g}$. Signal conditioning for each of the analog sensor outputs comprises a second order Sallen-key low pass filter (cut-off frequency $723 \mathrm{~Hz}$ ) for anti-aliasing.

A radio frequency communication link is provided for an operator to send in commands. Off-the-shelf 433MHz ASK transmitter (TX) and receiver (RX) modules are used in conjunction with the UART within PSoC ${ }^{\circledR}$. Both ends use $19 \mathrm{~cm}$ monopole antennae. The transmitter sends a moving average filtered version of the motor torque reference set by a sliding potentiometer. Only the receiver resides on the Chatur board. The transmitter is implemented using the ASK TX module and a general purpose PSoC board ${ }^{\S}$. Caveats: (1) Even though nominally equal clock frequencies are used at TX and RX, care should be taken to use same valued load capacitors on the crystals on both ends, else the frequency at TX can be slightly off from that at RX leading to communication errors. (2) A silent period of about $5 \mathrm{~ms}$ is added between consecutive samples to avoid the wrong bit being taken as start bit.

PWM pulses (from Chatur board to CIPOS $^{\text {TM }}$ boards) and fault signals (from the

\footnotetext{
${ }^{\S}$ Designed by Sudharshan Kaarthik, the author's lab-mate
} 
Table 4.6: Specifications of flyback power supply.

\begin{tabular}{ll}
\hline Input & $23-27 \mathrm{~V}, 0.6 \mathrm{~A}$ \\
\hline Outputs & (1) $5 \mathrm{~V}, 0.3 \mathrm{~A}$ (for all circuits on control side ground) \\
& (2) $5 \mathrm{~V}, 0.3 \mathrm{~A}$ (for low voltage circuits on power side ground) \\
& (3) $15 \mathrm{~V}, 0.3 \mathrm{~A}$ (for CIPOS ${ }^{\top \mathrm{M}}$ gate drive) \\
\hline Switching frequency & $100 \mathrm{kHz}$ \\
\hline
\end{tabular}

CIPOS $^{\text {TM}}$ boards to Chatur board) are opto-isolated (using 6N137). Potentiometers, indicator LEDs, push-button switches and pull-downs for current DACs (of PSoC ${ }^{\circledR}$ ) are provided for testing and debugging purpose. $\mathrm{PSoC}{ }^{\circledR}$ programming is done through a 10-pin JTAG interface using the MiniProg3 programmer.

The power supply for Chatur board is a multi-output flyback converter (Mohan et al., 2005; Umanand, 2009). It also supplies for the gate drive circuit and the fault detection circuit on CIPOS ${ }^{T M}$ board. It has three isolated outputs and switches at $100 \mathrm{kHz}$. The circuit schematic for the power supply is given in Figure B.15. A PWM controller (UC3845) generates the gate pulses for the MOSFET (IRF540). An RC snubber is used for protecting the MOSFET against voltage spikes at turn off. The flyback transformer is wound on a 36/22 ferrite pot core. SWG26 enamelled copper wire is used. The MATLAB/octave script for designing the flyback transformer is given in Listing B.1. Each output winding of the flyback transformer is followed by an LC filter, a common-mode choke and a linear regulator. Specifications of the flyback power supply are given in Table 4.6. Caveats: (1) Even though the chosen core size and shape is correct, the ferrite material used should be capable of $100 \mathrm{kHz}$ operation. Otherwise high core losses can lead to the flyback transformer running hot. (2) Common mode noise at the output needs to be reduced by using an inter-winding shield in the flyback transformer and a common-mode choke at each output.

『During early stages of testing, the controller board got burnt due to lack of isolation! 


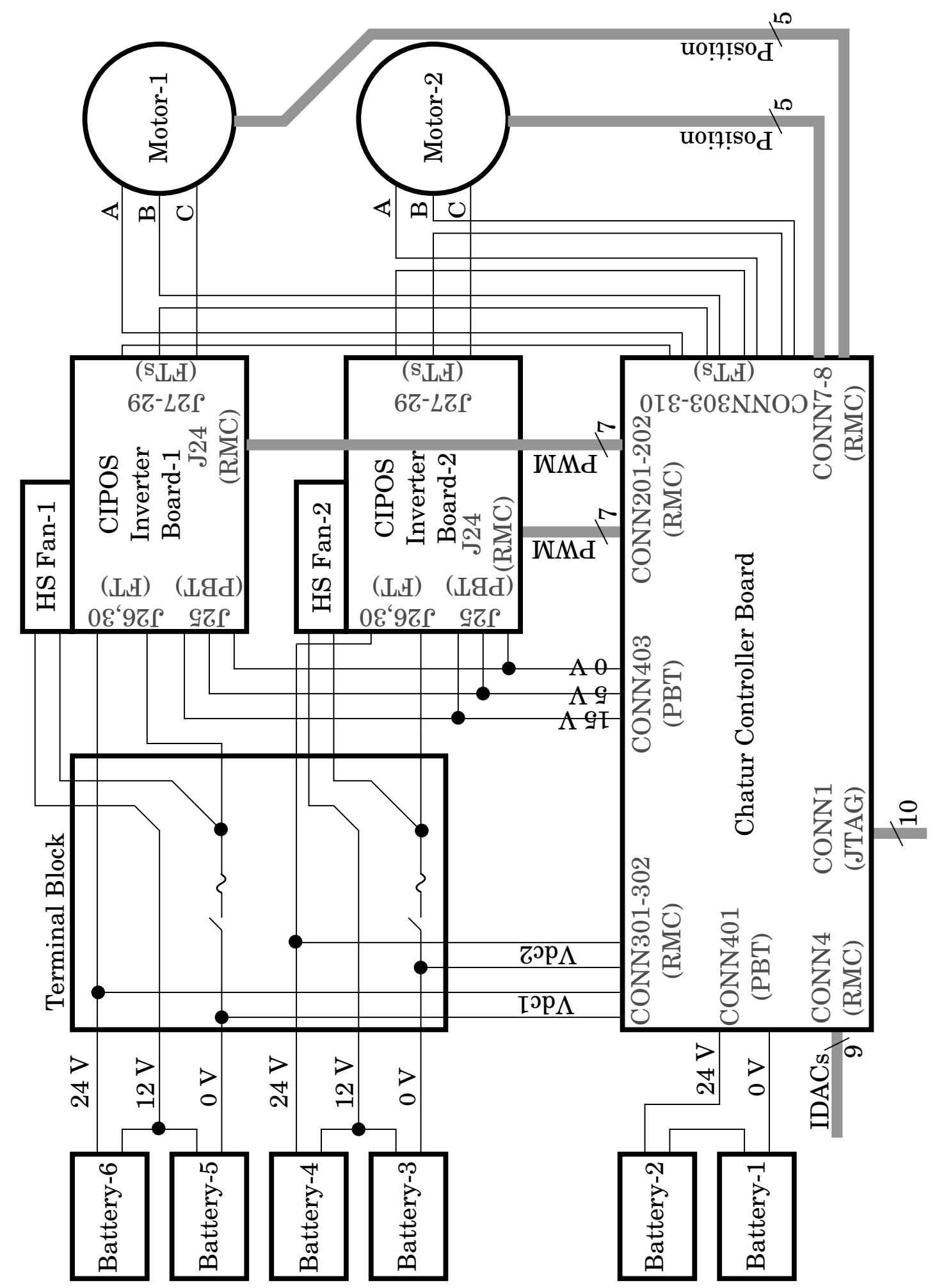

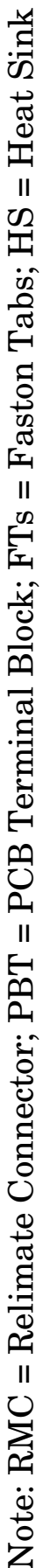

Figure 4.8: Electrical wiring diagram. 


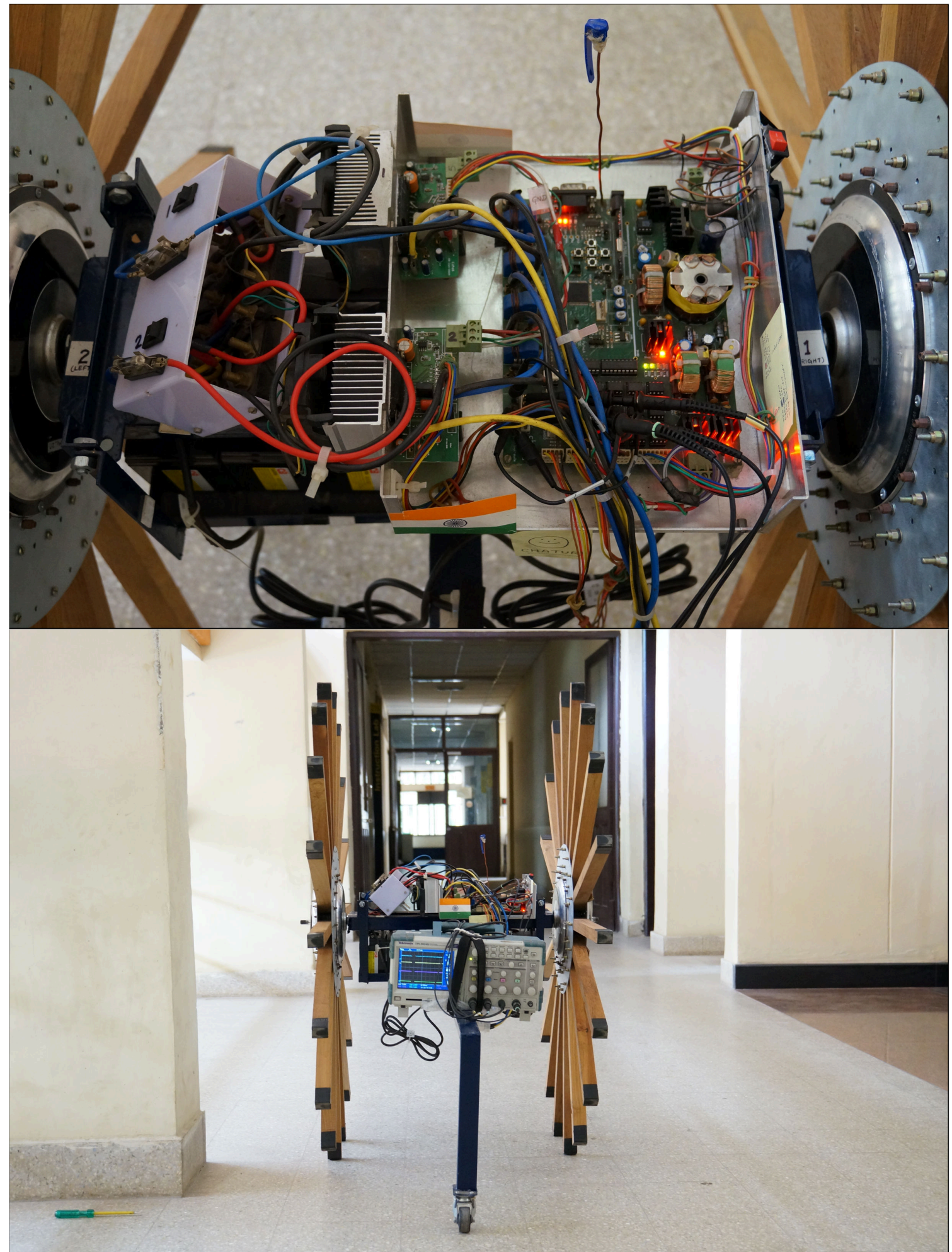

Figure 4.9: Full system after integration. Chatur is a fixed step-angle 2D dynamic walker implemented using two contralateral rimless spoked wheels. Each wheel is actuated by a BLDC hub motor with 18 equidistant spokes attached to the outer rotor. The frame contains the batteries and the electronics for power, control and measurement. A battery-operated fourchannel oscilloscope is mounted onboard to capture waveforms. 


\subsubsection{System integration}

Figure 4.8 shows the electrical wiring diagram of the complete system with connector details. Since the system is subjected to vibrations that could loosen friction based connectors, automobile grade locking connectors are used for the power and signal connections between motor and the PCBs. The mechanical and electrical subsytems are integrated to form the full mobile robot system called Chatur. The effective spoke length ( $l$; from center of motor to end of spoke) is $0.57 \mathrm{~m}$ and the inter-spoke angle $\left(2 \phi_{m}\right)$ is $20^{\circ}$. The full system mass $(m)$ is about $50 \mathrm{~kg}$. After integrating the mechanical and electrical subsytems of Chatur, the full system is shown in Figure 4.9.

\subsubsection{Load and actuator torque profile}

The rated motor current $\left(I_{\max }\right)$ is $12 \mathrm{~A}$ and the motor torque constant $\left(K_{t}\right)$ is $0.6 \mathrm{Nm} / \mathrm{A}$. Hence, the maximum actuator torque (combined effect of both motors driven by rated current) is given by $2 I_{\max } K_{t}=14.4 \mathrm{Nm}$. If the motor is at zero velocity at the lowest potential energy position, it needs a raising torque of $m g l \sin \phi_{m}=48.5 \mathrm{Nm}$. Hence, Chatur cannot raise itself from stand still in this position. Owing to this torque limitation of the motor, initial raising of the CoM is done manually and waveforms are taken for steady state forward motion.

Typical motor drive systems deal with mechanical loads that are defined in terms of three components: (1) A load torque term $\left(\tau_{l}\right)$ obtained from the torque-speed static characteristics, (2) A dynamic torque term $\left(J \frac{d \omega}{d t}\right)$ that accounts for the rotational acceleration overcoming inertia, and (3) A speed-dependent loss term $(B \omega)$ that accounts for viscous friction losses. The mechanical load on the motor in this rimless wheel system, unlike in a conventional wheel system, has a load torque that varies with the angle made by the stance spoke with the vertical $(m g l \sin \phi)$. Also, unlike typical loads, the system has the capability to convert its gravitational potential energy to kinetic energy because of which it is possible to actuate the system with a torque magnitude much smaller than the peak load torque. Figure 4.10 depicts the load and actuator torque profile for one stance phase with an inter-spoke angle of $20^{\circ}$ (inset). The same profile is repeated eighteen times in one mechanical revolution of the rimless wheel. Though the system is not self starting, once the CoM is manually raised, the actuator torque pulse just needs to overcome the losses in the system to keep it going forward. 


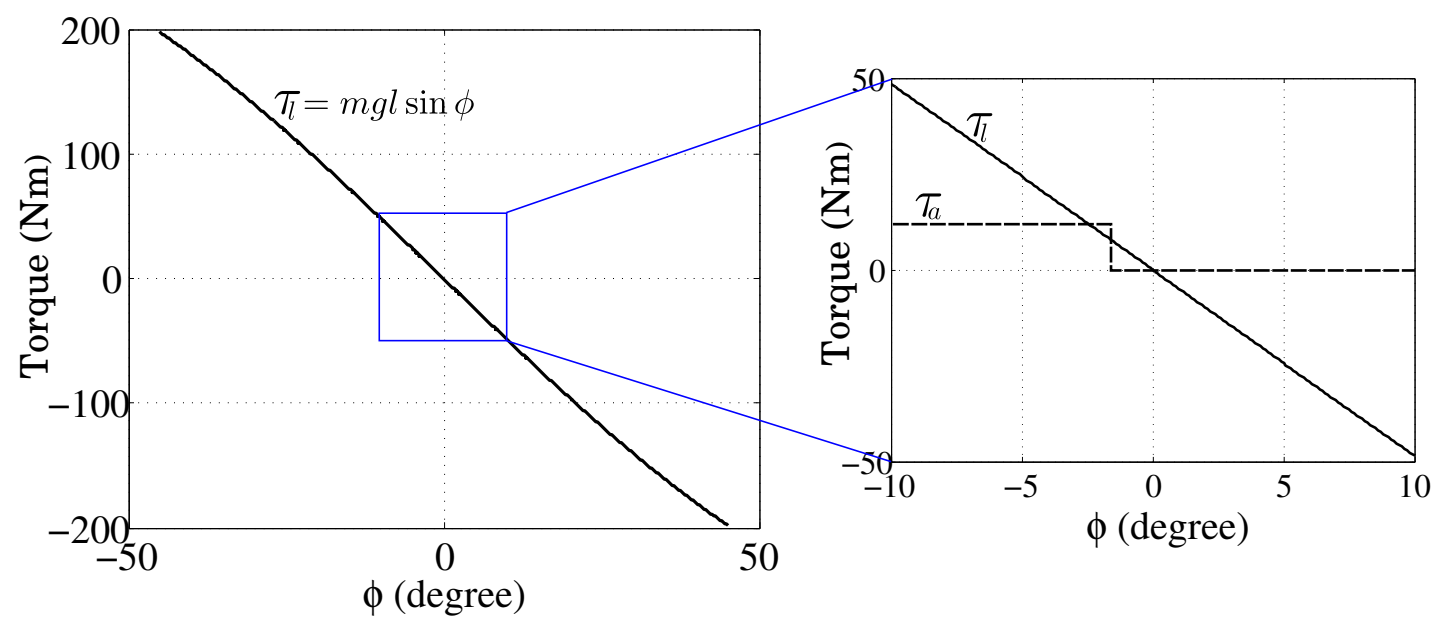

Figure 4.10: Torque profile for the opposing load torque $\left(\tau_{l}\right)$ of the rimless wheel varies sinusoidally with angular position of the stance spoke with respect to vertical. The present setup uses an inter-spoke angle $\left(20^{\circ}\right)$. The resulting variation of the load torque and the pulsed actuator torque $\left(\tau_{a}\right)$ over one stance phase is shown inset. The same pattern repeats eighteen times in one mechanical revolution of the motor.

\subsection{Control topology}

This section proposes a control topology for Chatur and describes its implementation using the $\mathrm{PSoC}^{\circledR} 5$ controller. The control scheme has two hierarchical levels:

1. Inner loop: torque control of BLDC motor

2. Outer loop: torque reference generation

Each of these tasks is implemented using hardware blocks in conjunction with embedded software running within the $\mathrm{PSoC}{ }^{\circledR}$. Hence, the control scheme implementation comprises two parts:

1. A top-level schematic that configures blocks of digital and analog resources available within $\mathrm{PSoC}^{\circledR}$.

2. A control algorithm (an embedded software) that runs on the ARM core of PSoC ${ }^{\circledR}$.

The PSoC ${ }^{\circledR}$ port map as applicable on Chatur board (Table B.1), the top level PSoC ${ }^{\circledR}$ schematics for the project (Figures B.16-B.19) and the C-code for the control algorithm (Listing B.2) are provided in Appendix B. 


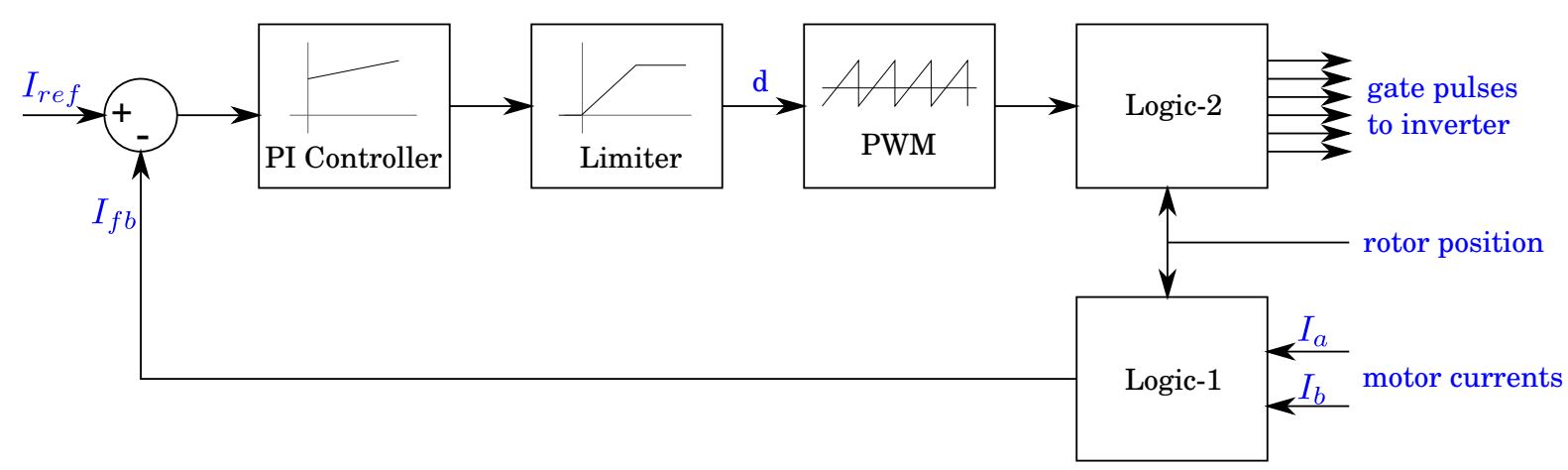

Figure 4.11: Block diagram of inner loop of control scheme: torque control of brushless dc motor. The duty ratio (d) input to the PWM block is the inverter pole voltage duty ratio (different from the pulsed torque duty ratio.)

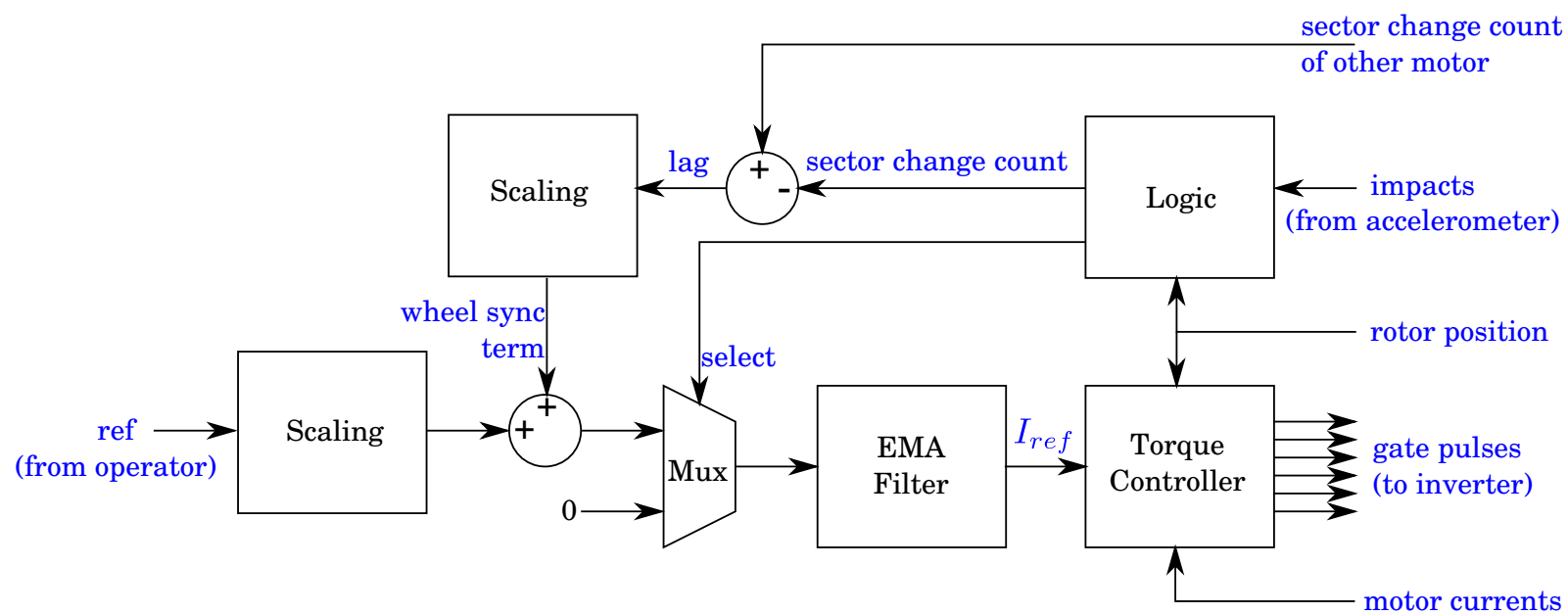

Figure 4.12: Block diagram of the full control scheme: inner loop (torque controller) and outer loop (torque reference generation).

\subsubsection{Torque control}

The heart of the control scheme is the torque control of the BLDC motor shown in Figure 4.11 (Patil et al., 2009). Since it is a permanent magnet motor" , torque control can be achieved by controlling the stator current. Based on the sensed rotor position, the torque feedback value is calculated from the sensed phase currents (Figure 4.13) and the right pair of windings are excited as per Table 4.3. Thus in each $60^{\circ}$ (electrical) sector, the BLDC motor can be treated as a DC motor. The current control is done using a Proportional-Integral (PI) controller. Figure 4.11 shows the block diagram of the torque control loop for one BLDC motor. The second motor too has a similar control structure.

\footnotetext{
${ }^{\|}$The outer rotor is embedded with permanent magnets
} 
The PI controller gains $\left(K_{p}\right.$ and $\left.K_{i}\right)$ are tuned using Ziegler Nichols method. Care is taken to avoid integrator wind-up by latching the integrator output once it reaches an upper limit. The PI controller output is limited to avoid large duty ratios that could lead to higher than rated currents. The PWM block uses a ramp compare method to generate the gating pulses of the required duty ratio. The logic block (Logic-2) after the PWM block decides which pair of windings to excite and which to leave unused based on the sensed rotor position.

A hardware based method is employed for inverter fault checking. The fault signal coming from CIPOS $^{\top \mathrm{TM}}$ board is passed through a glitch filter followed by a latch the output of which gates all the PWM outputs. Software based fault checking (polling, interrupts) should be avoided as it is slow and unreliable. Spurious trips caused due to noise on the fault signals are eliminated by using hardware glitch filters (glitch length $4.9 \mu \mathrm{s}$ ). Noise issues could also introduce glitches in sector identification. These glitches are eliminated by using a hardware glitch filter (glitch length $600 \mu \mathrm{s}$ ) in $\mathrm{PSoC}^{\circledR}$.

\subsubsection{Current feedback}

Figure 4.13 illustrates the nominal current waveforms (neglecting switching frequency artifacts) for a three phase BLDC motor. Typically, the time constant of the current rise and fall is a much smaller fraction of the sector dwell time than what is indicated in the figure.

Taking a commutating phase current as the torque feedback leads to current overshoots at sector transitions. This is because the feedback value is low during the commutation when the current magnitude is still rising. The controller tries to correct for this perceived drop in torque leading to a spike in current that can trip the inverter. Hence, the phase current that best conveys the information of the motor torque is the one that is not commutating in the sector transition. The torque feedback is thus reconstructed from the non-commutating current in each sector. Various position sensorless current commutation techniques exist for BLDC motors (Chen and Liaw, 1999; Chi et al., 2013). But most of these methods rely on the sensed back emf which is very low $(<1 \mathrm{~V})$ and hence unusable in the present application.

Figure 4.14 depicts the strategy used for obtaining the motor currents once in every PWM cycle. The measured motor currents $\left(I_{a}\right.$ and $\left.I_{b}\right)$ obtained from the current sensors are relayed to an 8-bit SAR ADC through an analog multiplexer available within $\mathrm{PSOC}^{\circledR}$. 


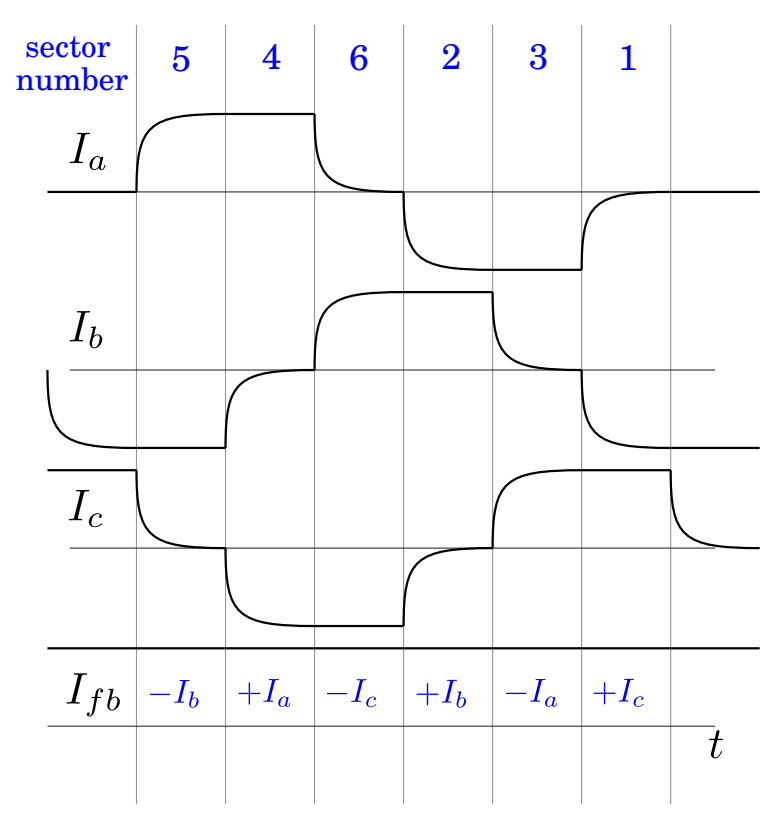

(a) CCW rotation

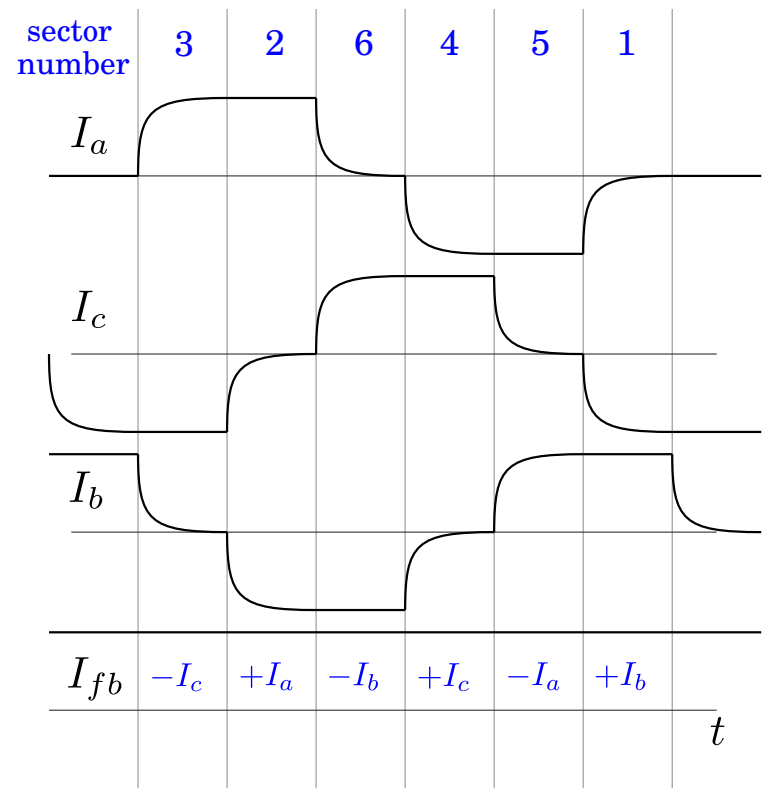

(b) CW rotation

Figure 4.13: Reconstructing torque feedback $\left(I_{f b}\right)$ from BLDC motor phase currents. At each sector transition, the magnitude of the non-commutating phase current is taken as $I_{f b}$.

The multiplexer alternately switches between $I_{a}$ and $I_{b}$ every $30.6 \mu$ s (half of the PWM cycle time). It can have a finite settling time after a channel switch happens. Hence, the start-of-conversion to the ADC is given after the multiplexer switches to a new channel and stays there for $15.3 \mu \mathrm{s}$ (half of the channel dwell duration of the multiplexer). The start-of-conversion to the ADCs in $\mathrm{PSoC}{ }^{\circledR}$ needs to be a level (maintained throughout the conversion) and not just an edge**. The ADC completes conversion in $10 \mu \mathrm{s}$ and writes the 8-bit words for the sensed currents into designated registers through DMA transfer, without interrupting the processor. This helps save processor cycles thus reducing software loop time. This method is used for getting $I_{a}$ and $I_{b}$ of both the motors (two separate ADCs).

The ARM processor picks up the latest digital words corresponding to the noncommutating current from the registers once every software cycle. It then rectifies, level-shifts, scales and filters it in order to get a torque feedback value. Rectification is required as the sensed current is negative in certain sectors. Level shifting gets rid of the DC offset of the sensor. Scaling translates the 8-bit value to a 16-bit current feedback value in 4.12 fixed point format. Filtering eliminates switching and high frequency noise artifacts from the measured current. It comprises a glitch filter followed by an exponen-

\footnotetext{
**This discrepancy resulted in wrong ADC output values in an early version of implementation.
} 

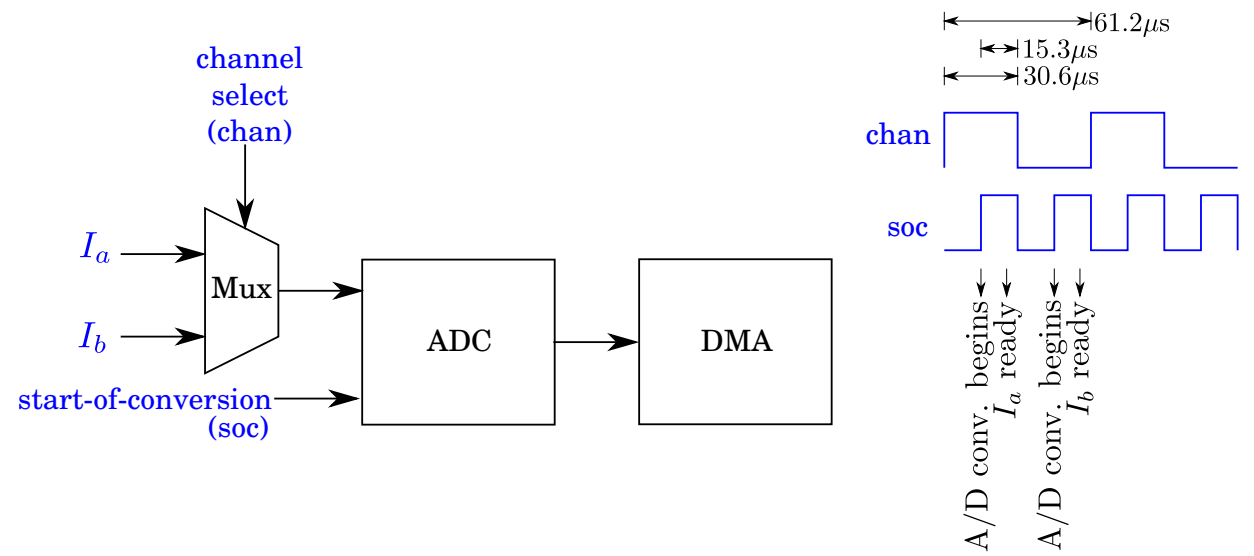

Figure 4.14: Strategy for obtaining motor currents once in every PWM cycle.

tial moving average filter. This glitch filter checks if difference between two consecutive samples is too large to be physically possible and retains the old sample value if so. The delay in sensing introduced due to the moving average filter should not be too large, else the current can reach its trip value before the controller could act. In Figure 4.11, the logic block (Logic-1) after the current sensors incorporates all the above tasks for computing the current feedback depending on the sector number.

\subsubsection{Torque reference generation}

In order to obtain a pulsed actuation torque, the torque reference $\left(I_{r e f}\right)$ needs to be shaped depending on the intended regime of walking and the corresponding spatial duty ratio. For instance, in case of regime 2 walking, a positive step in $I_{r e f}$ is introduced at ground impact and then reduced back to zero upon reaching mid-stance.

The accelerometer output $\left(a_{z}\right)$ is checked at the start of each software cycle. Whenever $a_{z}$ crosses a threshold, a ground impact is detected and a positive step is introduced in $I_{r e f}$. The magnitude of the torque step is set internally or controlled by the operator via the radio control. Depending on the intended regime of walking, $I_{\text {ref }}$ is reduced back to zero after the desired number of sector changes in motor position. Between two consecutive impacts, the wheel (and hence the motor) sweeps through $20^{\circ}$. As sector change in position sensors happens every $360^{\circ} /(25 \times 6)=2.4^{\circ}$, there are eight sector changes in a stance phase. The processor keeps track of the number of sector changes following an impact and uses this information to time the removal of the torque pulse. For instance, a nominal regime 2 walk requires the removal of the torque pulse after four sector changes following a ground impact. This gives a spatial duty ratio $\left(d_{i s}\right)$ of $4 / 8$ i.e. 0.5. 
Table 4.7: Time scales of various events/processes.

\begin{tabular}{ll}
\hline Description & Time \\
\hline ADC conversion time & $10 \mu \mathrm{s}$ \\
PWM cycle time & $61.2 \mu \mathrm{s}$ \\
Software loop time & $100 \mu \mathrm{s}$ \\
Motor $L / R$ time constant & $350 \mu \mathrm{s}$ \\
Sector dwell time & $30-120 \mathrm{~ms}$ \\
Double impact blindfold window & $300 \mathrm{~ms}$ \\
Step duration & $350-900 \mathrm{~ms}$ \\
\hline
\end{tabular}

Figure 4.12 shows the block diagram of the full control scheme. The reference value commanded by the operator is used as the step size of the torque pulse. The select line for the multiplexer controls the torque duty ratio and hence the regime of walking. The multiplexer output is filtered (exponential moving average) before being passed on as the torque reference.

Chatur's steady state speed settles at the value where the energy losses equal the energy put in by the actuator. The losses are likely to change from step to step because of physical asymmetries and non-uniformities in inter-spoke angle, shape of the foot point and hardness of the surface of travel. Hence, the steady state speed is only nominally constant. Large variations in speed can be compensated for by manually changing the reference value sent via radio control.

\subsubsection{Wheel synchronization}

Owing to practical asymmetries, there is a possibility of the two wheels going out of sync. In such a case, the ground impacts of corresponding spokes on the two wheels are time displaced leading to a double impact as opposed to a single impact (see Figure 4.17b). In such a case, the number of sector changes in a stance phase is smaller for one motor and larger for the other. In the subsequent stance phase (duration between two ground impacts when the rimless wheel vaults over one spoke), the motor that is lagging is given a slightly higher torque reference and the one that is leading is given a corresponding lower torque reference. Wheel synchronization is achieved in this way. The scaling factor $\left(K_{\text {sync }}\right)$ for the wheel sync term is tuned by trial and error.

The flowchart for the full control scheme of Chatur is given in two parts in Fig- 
ures 4.15-4.16. Immediately following a ground impact, a double impact blind-fold window $(300 \mathrm{~ms})$ is introduced. During this interval the processor is blind to any further impacts. This ensures that the second impact in a double impact event is not interpreted as a fresh ground impact that resets the sector change counts. The software loop time can vary (80-90 $\mu \mathrm{s})$ due to 'if' and 'case' statements in the code. Varying software loop time changes the effective controller gains. Hence, the loop time is fixed to $100 \mu \mathrm{s}$ by using a timed interrupt. After all the computation in a software iteration is done, the processor remains in a WAIT state till the periodic interrupt arrives. Only then is the the next iteration resumed. The time scales of the various events/processes in the system are collated in Table 4.7. 


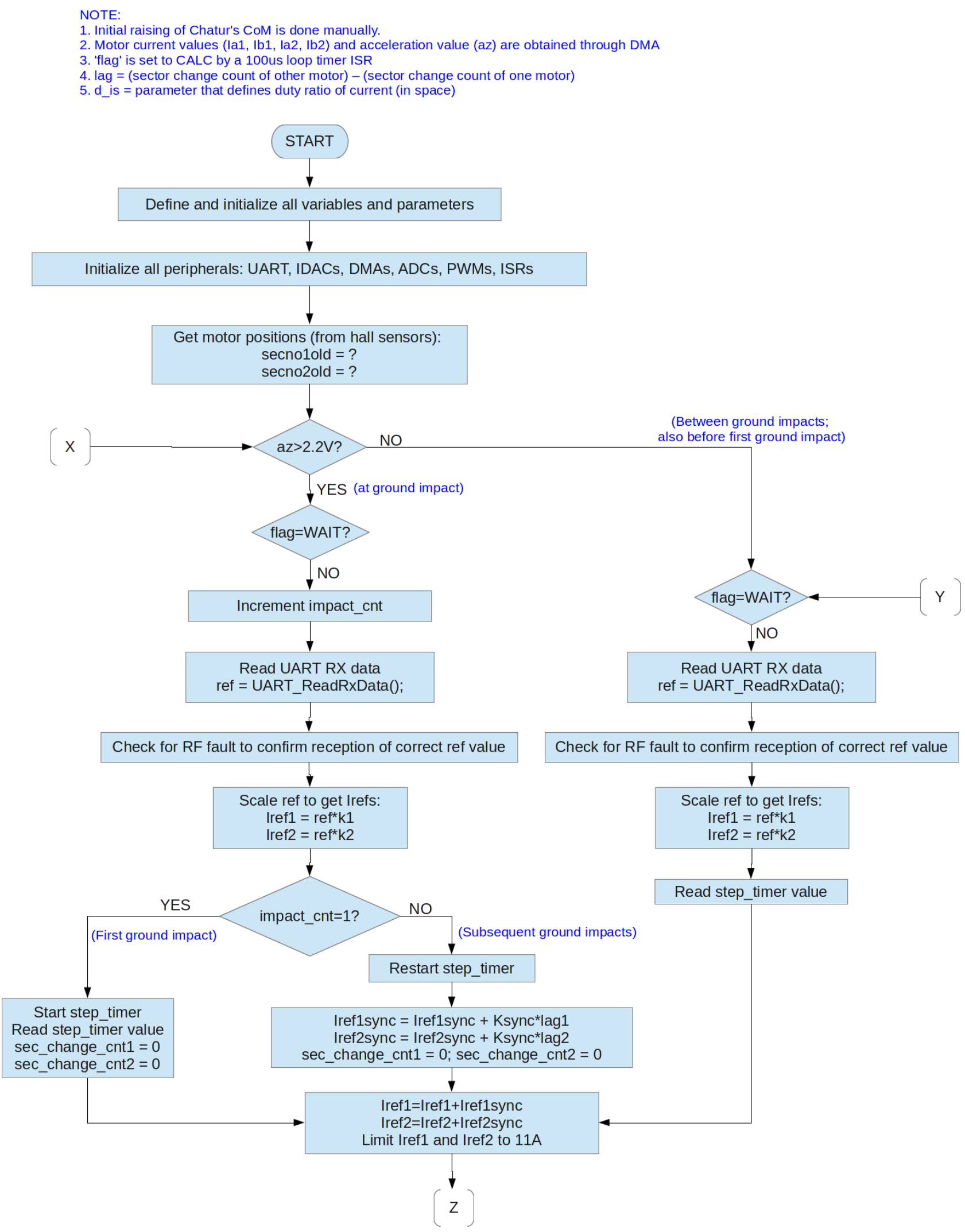

Figure 4.15: Flowchart of full control scheme (1/2). 


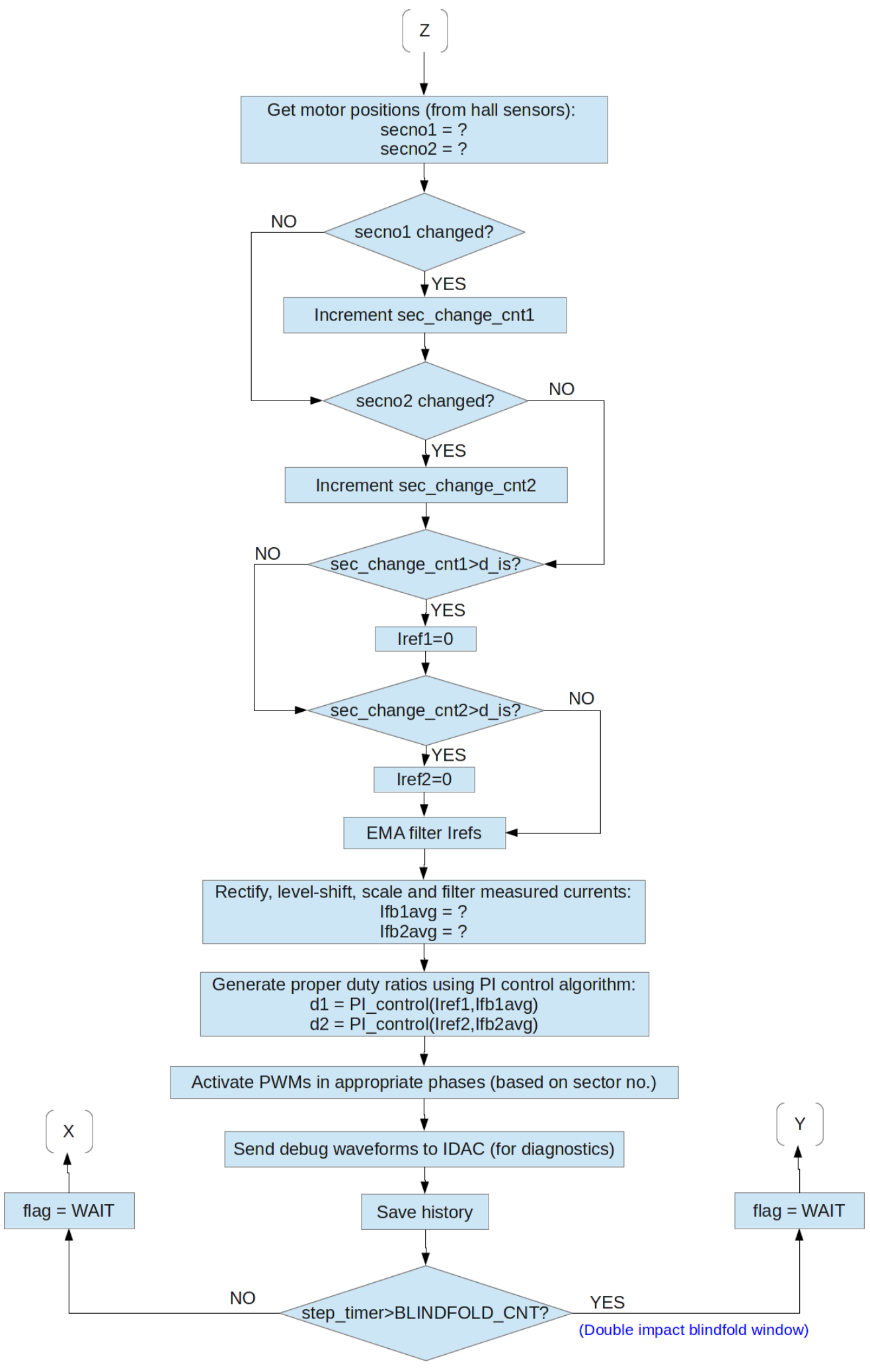

Figure 4.16: Flowchart of full control scheme (2/2). 


\subsection{Experimental results}

This section gives the experimental results in terms of the working of the control scheme - torque control, wheel synchronization and steady state forward motion of Chatur using suitable waveforms.

At the instant of ground impact, detected by an impulse in the accelerometer output (Figure 4.17a), the torque reference is raised to a value set by the operator. The torque reference is brought back to zero after a predefined number of sector changes following the ground impact. The waveforms show that the torque feedback tracks the torque reference. One of the motor phase currents is also shown for reference.

Figure $4.17 \mathrm{~b}$ illustrates a double impact identified by a double impulse in the accelerometer output. Owing to a finite lag between the two motors, the wheel synchronization loop kicks in to change the torque references of the two motors (see Figure 4.12) thus bringing the wheels back in sync on subsequent ground impacts. The torque duty ratio in this set of waveforms is kept at $100 \%$ (continuous actuation; regime 4) to make it easier to notice the dip in the torque reference of one of the motors. The sector number of motor- 1 is seen to follow the cyclic sequence 5-4-6-2-3-1 (CCW rotation). The waveforms for steady state forward motion of Chatur in all four torque regimes are depicted in Section 2.6. Waveforms for two different average speeds achieved by changing the torque duty ratio are reproduced here (Figure $4.17 \mathrm{c}, \mathrm{d}$ ). 


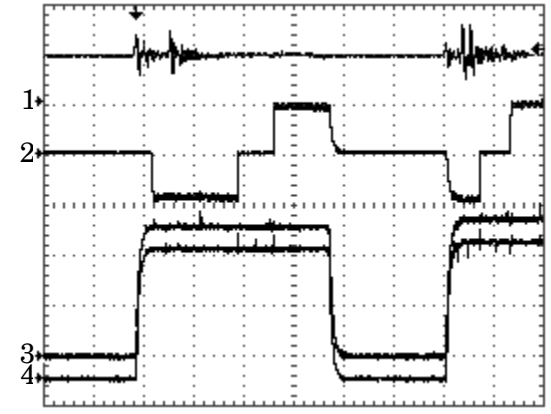

(a) Torque control: torque feedback is seen tracking the pulsed torque reference.

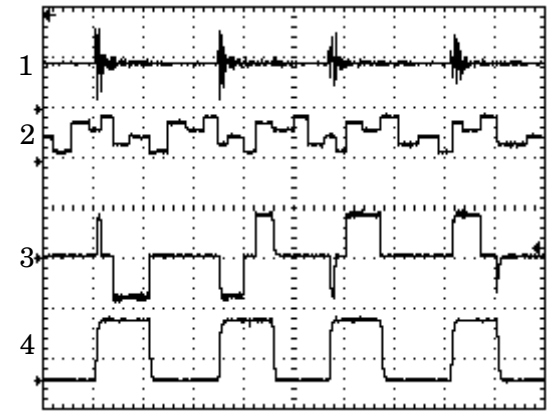

(c) Duty ratio $<0.5, v_{\text {avg }}=0.33 \mathrm{~m} / \mathrm{s}$.

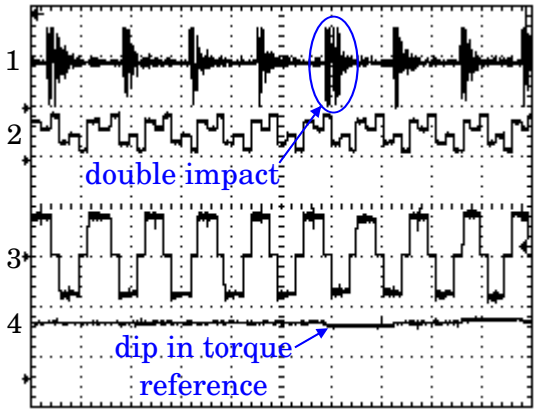

(b) Wheel sync: double impact causes a dip in torque reference for one motor.

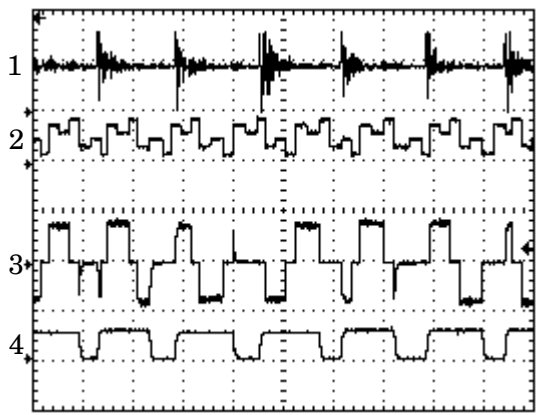

(d) Duty ratio $>0.5, v_{a v g}=0.49 \mathrm{~m} / \mathrm{s}$.

Figure 4.17: Waveforms validating the working of the control scheme: (a) pulsed torque control, (b) wheel synchronization, (c,d) steady state forward motion of Chatur. Scales: (a) (1) Accelerometer output: $2 \mathrm{~V} /$ div, (2) Motor phase current: $10 \mathrm{~A} /$ div, (3) Torque reference: $0.5 \mathrm{~V} /$ div, (4) Torque feedback: $0.5 \mathrm{~V} /$ div, time scale: $100 \mathrm{~ms} /$ div. (b,c,d) (1) Accelerometer output: $2 \mathrm{~V} /$ div, (2) Motor-1 sector number, (3) Motor-1 phase current $I_{a 1}: 10 \mathrm{~A} / \operatorname{div}$, (4) Torque reference: $1 \mathrm{~V} / \mathrm{div}$ in (b,c) and $2 \mathrm{~V} / \mathrm{div}$ in (d), time scale: $250 \mathrm{~ms} /$ div.

\subsection{Conclusion}

This chapter describes the hardware design and proposes a control topology for pulsed torque actuation of two BLDC hub motors in a dual-rimless-wheel walker with a characteristic cyclic variation in reflected load torque. Experimental waveforms validate the working of the control scheme in terms of torque control, wheel synchronization and steady state forward motion of the mobile robot. An improved BLDC drive is used that reduces current overshoots during sector transitions by using non-commutating current feedback. The magnitude and duration of the applied torque pulse is programmable by the user and enables attainment of various steady state speeds. 


\section{Chapter 5}

\section{Conclusion}

This chapter summarizes the research contributions in terms of the key findings and their implications. The scope for further work is also suggested in the mathematical as well as physical models.

\subsection{Summary of contributions}

1. Closed-form analytical solution for stance phase dynamics of inverted pendulum walking: A simple closed-form analytical solution, using hyperbolic functions, is proposed for the stance phase dynamics of $2 \mathrm{D}$ inverted pendulum walking. In addition to gravity, constant actuation and braking torques expressed in a parametric form, are taken into account. The proposed solution (analytical) approximates the exact one (numerical) within $6 \%$ upto a step angle of $25^{\circ}$. On account of being analytical, as opposed to numerical, the proposed solution is a useful tool for investigating the effect of various parameters and variables on the stance phase dynamics of any inverted pendulum based walker.

2. Torque regimes in a rimless wheel based walker using pulsed torque actuation: A pulsed actuation torque is proposed for the rimless wheel based 2D dynamic walker resulting in four torque regimes defined by the ratio of energy losses to available actuator torque. Higher the losses, more the pulse duty ratio required for a steady-state walk. A stance phase can have four types of sub-phases - unactuated rise, unactuated fall, actuated rise, actuated fall. These are concatenated in four different ways to form repeating cycles yielding the four regimes. This work 
considers pulsed torque of constant magnitude. More generic walkers can have time varying actuator torques. But the basic idea of the actuation being present only for a portion of the stance phase still holds. Hence the proposed regimes with the defined repeating cycles can be observed in all walkers - engineered or biological.

\section{Framework of physical constraints on the choice of operating points in} inverted pendulum walking: The operating point of the inverted pendulum walker is specified by a combination of initial mid-stance velocity $\left(v_{0}\right)$ and step angle $\left(\phi_{m}\right)$ chosen for a given walk. Not all operating points lead to a realizable steadystate gait. Using basic mechanics, a framework of physical constraints that limit the choice of operating points is proposed. These constraints are the fundamental limits on walking like an inverted pendulum. The constraint lines thus obtained delimit the valid region of operation of the walker in the $v_{0}-\phi_{m}$ plane. Within this allowable region, sub-regions that result in various regimes of walking are identified. The magnitude of the available actuation torque affects the shape and size of the valid region of operation. Operating points of all inverted pendulum walkers must fall within this valid region.

4. Optimal operating points in inverted pendulum walking: For any given speed, the optimal operating point that ensures minimum mechanical power consumption is located based on tangency of the power and velocity contours. Repeating for different speeds, the locus of optimal operating points is obtained. The shape and location of this optimal locus is sensitive to the models chosen for loss and internal energy. Using an appropriate constant step angle over a broad range of speeds could lead to an inverted pendulum walker that is close to optimal. This optimality is different from the optimality based on minimum metabolic cost and is more relevant for engineered walkers rather than biological walkers.

\section{Hardware design and control topology for pulsed torque actuation of} a synchronized dual BLDC motor driven platform: The hardware design for the mechanical and electrical subsystems of Chatur are elaborated. To avoid falling sideways, the system uses two adjacent wheels driven by separate BLDC hub motors. The reflected load torque has a characteristic cyclic variation repeating several times within a mechanical revolution. The proposed control topology has two hierarchical levels, an inner loop for torque control of BLDC motor, and an outer loop for torque reference generation. Ground impacts of the spokes are detected by an accelerometer to initiate the application of torque. Wheel synchronization is 
achieved by incrementing the torque of the motor that is lagging. The BLDC motor drive uses non-commutating current feedback to reduce current spikes during sector transitions. The proposed control topology, with suitable ad hoc modifications, can be used in other synchronized dual motor systems with pulsed actuation.

\subsection{Scope for further work}

\section{Mathematical model}

1. A unified mathematical model for walking and rolling needs to be arrived at. Substituting a step angle of zero in the model should produce the model of a rolling wheel of radius equal to the chosen leg length. To make such a model possible, a particular type of surface of travel and type of foot has to be assumed. The stance phase during walking involves rolling over the stance foot as the center of pressure moves from the toe-end to the heel end of the foot. A suitable rolling resistance model needs to be devised that holds for walking as well as rolling.

2. The model presented in this work neglects the contribution of the internal energy $\left(E_{\text {int }}\right)$ to the dynamics and considers its effect only on the energetics. Effect of distributed mass, as opposed to a lumped mass, needs to be incorporated in the mathematical model. Consequently, the effect of $E_{\text {int }}$ on the dynamics of the walker can be investigated.

\section{Experimental model}

1. Losses need to be reduced in order to make the experimental prototype more energy efficient. The impact losses can be reduced by designing a suitable compliance into the leg. The braking losses can be reduced by better wheel alignment (lesser toe-in and camber) and by using motors with lower detent torque. Actuators with high efficiency in the low speed region $(0.4-10 \mathrm{rad} / \mathrm{s}$ i.e. $4-10 \mathrm{rpm})$ need to be used.

2. The experimental model needs to be augmented with the ability to start from standstill. This can be done by increasing actuator torque or introducing some mechanical advantage. One solution is to use actuated feet as opposed to hip actuation. That would require a large number of motors (one per spoke) of lower torque rating. Another solution is to use spokes of variable length which again requires additional linear actuators. 
3. In order to avoid the undesirable effects of the castor wheel on the dynamics of the system, the tail piece needs to be done away with. Reducing the overall system weight and using a suitably positioned counterweight on the stator can help in this regard. An additional benefit is the ability to walk forward as well as backward.

4. Chatur is a fixed step-angle dynamic walker owing to the constant inter-spoke angle of the rimless wheel. A mechanism of varying the step-angle needs to be incorporated. This will enable spanning a larger set of operating points in the $v_{0^{-}}$ $\phi_{m}$ plane raising the possibility of an optimal walker that always tries to be on the optimal locus as discussed in Section 3.2.

5. Instead of keeping the rimless wheels in sync, it is possible to achieve straight line walking with two rimless wheels that are phase displaced in their motion. The spokes of the left and right wheel would hit the ground alternately bringing the model in closer resemblance to a 3D walking biped. To enable this, the distance between the wheels has to be smaller than that in the present set-up. The walker will sway from side to side i.e. there will be some lateral dynamics which will have to be taken into account.

6. The present work investigates the dynamics and energetics of $2 \mathrm{D}$ motion. With the rimless wheels moving at different speeds, it is possible to achieve turning albeit with large reflected torque on the actuators. The dynamics and energetics of turning needs to be investigated and suitable techniques to achieve it with minimal energy expenditure needs to be developed. 


\section{Appendix A}

\section{Jansen Mechanism}

Theo Jansen designed a planar linkage mechanism that mimics the leg motion pattern of a walking mammal (Figure A.1). It is a 12-link mechanism including a crank link that is actuated by a rotary actuator and a stationary link that is part of the chassis of the legged system. The stationary link - between the crank center and a pin joint - is shown in dashed line and is inclined at an angle of $11.6^{\circ}$ to the horizontal. All other links and joints are unactuated and move because of the motion imparted by the crank. Their positions and orientations are uniquely defined by specifying the crank angle and hence the mechanism has only one degree of freedom (1-DoF). The link lengths as designed by Jansen are given in Table A.1 where the link numbers are as shown in Figure A.2a.

\section{A.1 Forward kinematics}

Determining the motion of every point on the mechanism from known geometry is called forward kinematics. Points where the links are pin jointed are referred to as nodes. Using circle intersection method, Ingram (2004) determined the trajectories of various nodes (Figure A.2a) and link CoMs (Figure A.2b) in the chassis reference frame. The foot point trajectory forms a closed shape whose horizontal extent gives the step length and vertical extent gives the step height (Figure A.2c).

The end of the crank (node-0) and the foot point (node-5) trace their respective trajectories in the same sense. If the crank rotates clockwise, the foot point also traces its trajectory clockwise while the knee joint (node-4) goes anti-clockwise. In presence of a number of such legs in a walker, the landing and take-off points are annotated on the 
Table A.1: Link lengths in Jansen leg mechanism in proportion to crank length.

\begin{tabular}{ll}
\hline Link & Length \\
\hline Link-0 (crank) & $l_{0}=1.00$ \\
Link-1 & $l_{1}=3.33$ \\
Link-2 & $l_{2}=3.72$ \\
Link-3 & $l_{3}=2.63$ \\
Link-4 & $l_{4}=4.38$ \\
Link-5 & $l_{5}=3.27$ \\
Link-6 & $l_{6}=4.13$ \\
Link-7 & $l_{7}=2.45$ \\
Link-8 & $l_{8}=2.62$ \\
Link-9 & $l_{9}=2.77$ \\
Link-10 & $l_{10}=2.67$ \\
Link-11 (fixed) & $l_{11}=2.59$ \\
\hline
\end{tabular}

foot point trajectory. The foot point trajectory is almost flat between the landing and take-off points. Changing the frame of reference from the chassis of the walker to the ground, the trajectory of the crank center is quite flat. Hence the trajectory of the walker CoM (located in the chassis) is also flat.

In order to have a variable step length, one more degree of freedom needs to be added to the Jansen mechanism. This can be done by making any one of the links have a variable length by introducing a linear actuator in the link. The step length in Jansen mechanism is not very sensitive to change in the length of the stationary link. The effect of changing crank length on step length and step height is plotted in Figure A.2d. The variation in step length $\left(l_{s}\right)$ with crank length $\left(l_{0}\right)$ is found to be linear and is approximated by the following equation.

$$
l_{s}=4 l_{0}+0.5
$$




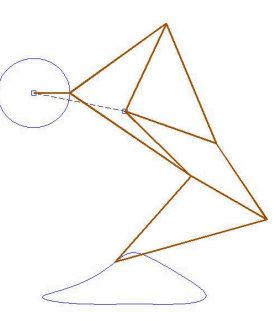

(a) crank angle $=0^{\circ}$.

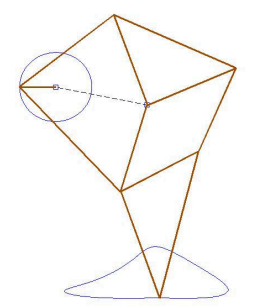

(f) crank angle $=180^{\circ}$.

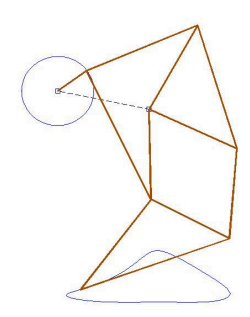

(b) crank angle $=36^{\circ}$.

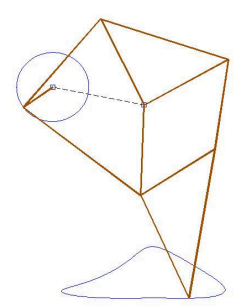

(g) crank angle $=216^{\circ}$
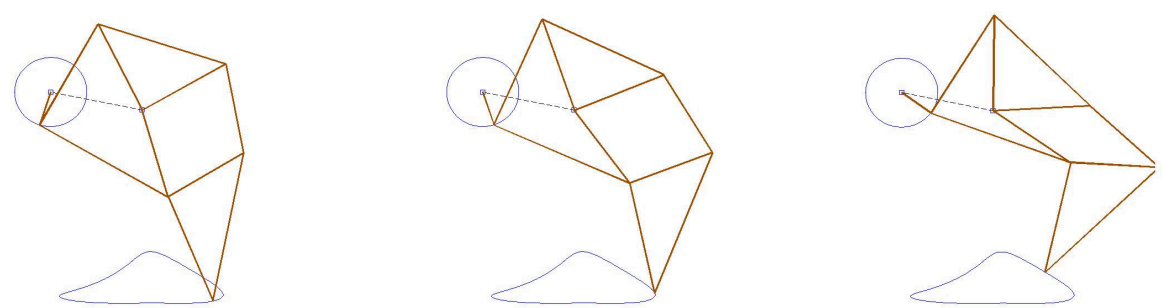

(h) crank angle $=252^{\circ}$.

(i) crank angle $=288^{\circ}$

(j) crank angle $=324^{\circ}$.

Figure A.1: Jansen leg mechanism at various crank angles. 


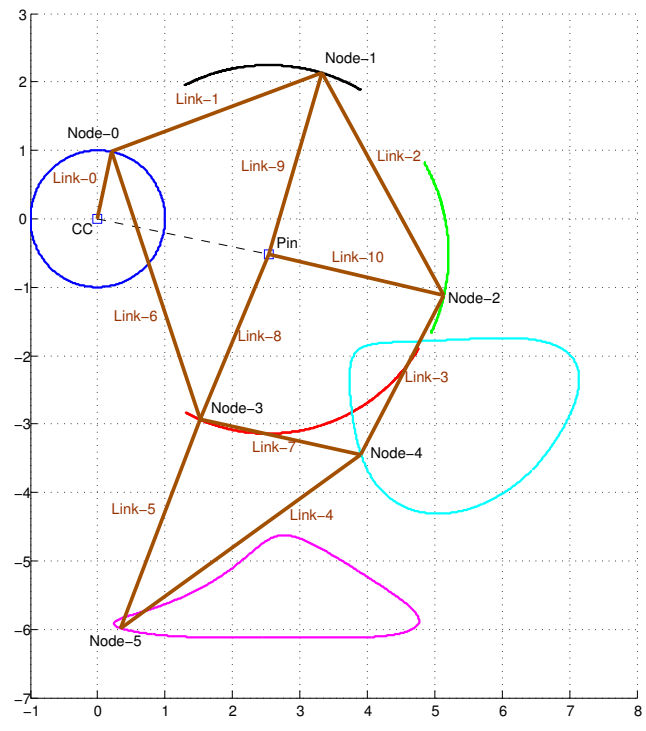

(a) Node trajectories.

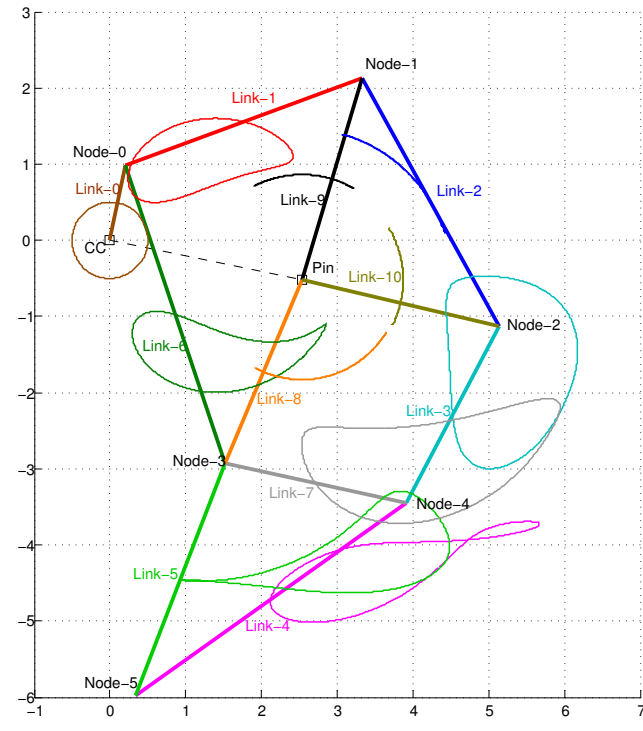

(b) Link CoM trajectories.

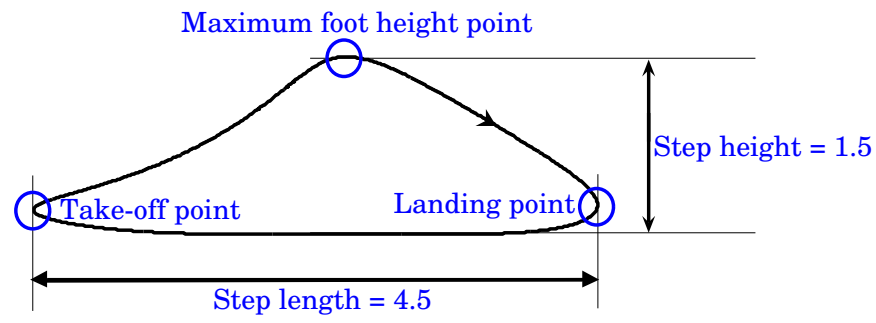

(c) Step length and step height.

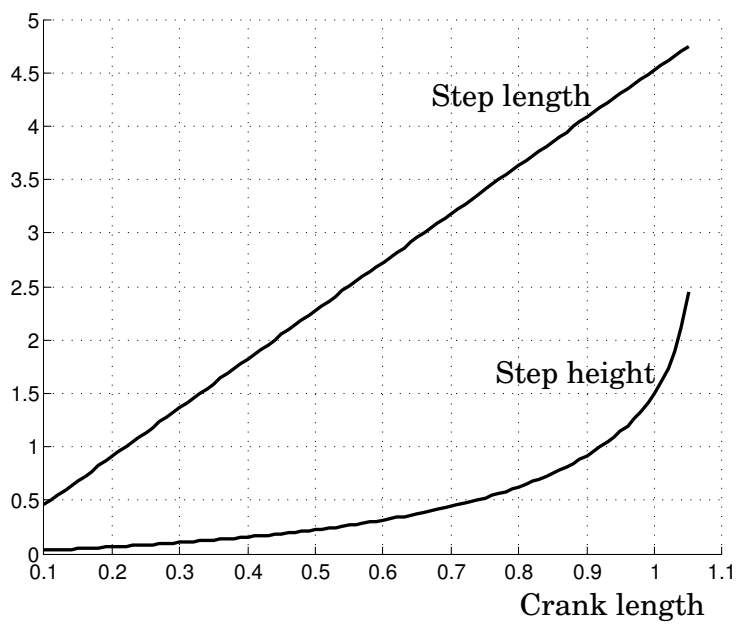

(d) Step length and step height with varying crank length.

Figure A.2: Trajectories of various points in Jansen leg mechanism and obtaining step length and step height from the foot point trajectory. Crank length $=1$ for (a), (b) and (c). 


\section{A.2 Inverse dynamics}

Determining internal forces and/or torques from the given motion of the elements and known external forces is called inverse dynamics. In order to obtain the design specifications of the motor and the links in the Jansen mechanism, inverse dynamics needs to be carried out assuming the crank rotates at a constant angular speed.

The dynamics of linkage mechanisms can be modelled using bond graphs (Karnopp and Margolis, 1979; Karnopp et al., 1990; Diwakar, 1996). The bond graph model for each link comprises multiport modulated transformers (mTF) that transform the forces at the ends of the links to forces acting on the link CoM and/or torques acting on the link. They also transform link end velocities to link CoM velocity and/or link angular velocity. The modulation coefficients in an $\mathrm{mTF}$ are a function of the angular position (variable) of the link and the geometry (fixed) of the link in terms of the relative position of its CoM with respect to the point of application of the force.

The simple links in Jansen mechanism - links $0,1,3,6$ and 8 - are assumed to have their CoM at the mid-point of the link. When three links form a triangle, it is a fixed structure incapable of relative motion between the links. Such links are considered as composite links — link 2-9-10 and link 4-5-7 — with their CoM located within the triangle. Let the frame of reference be fixed on the chassis as in Section A.1. Some of the links in Jansen mechanism - link 0, link 8 and link 2-9-10 - undergo pure rotation about a fixed point (Figure A.3). Their CoM trajectories are arcs of fixed radii. The bond graph model of such links is shown in Figure A.4. The other moving links - links 1, 3, 6 and link 4-5-7 — undergo rotation as well as translation (Figure A.5). The bond graph model of such links is shown in Figure A.6. If a link is subjected to more forces, further mTFs need to be added to these link models to incorporate the effect of those forces.

The interaction between the links happens at the pin jointed ends of the links. Putting together the models for all the links and that for a constant speed motor at the crank, the bond graph model for the complete mechanism is obtained (FigureA.7). Note that the joints between links are modelled as stiff springs with damping (compliance, $C=10^{-8}$; damping resistance, $R=0.001)$. This takes care of causality issues in the model. Some of the mTFs are not shown (between links 1 and 6; between links 4-5-7 and 6) in the figure for ease of viewing. All the bonds are numbered for ease of referencing. The angular velocities of links- $0,1,2,3,4,6,8$ are integrated with respect to time to obtain 


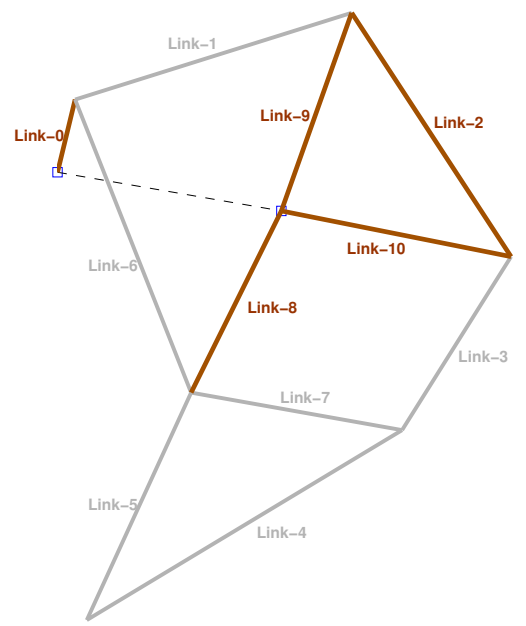

Figure A.3: Links undergoing pure rotation.

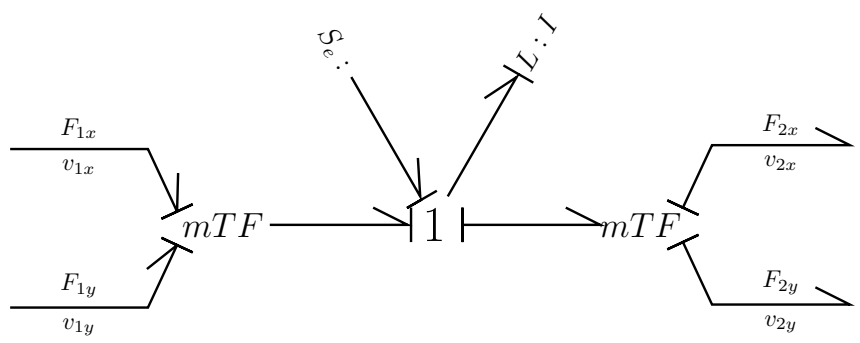

Figure A.4: Bond graph model for links undergoing pure rotation.

the respective angular displacements $q_{1}, q_{16}, q_{33}, q_{61}, q_{126}, q_{91}$ and $q_{110}$ (numbering as per bond numbers, not link numbers). Angular speed and hence displacement of links-9 and 10 are same as that of link-2. Angular speed and hence angular displacement of links-5 and 7 are same as that of link-4. The mTF coefficients are given by the following equations.

$$
\begin{aligned}
& M_{1}=M_{8}=\left[\begin{array}{ll}
-l_{0} \sin q_{1} & l_{0} \cos q_{1}
\end{array}\right] \\
& M_{2}=\left[\begin{array}{ccc}
1 & 0 & \frac{l_{1}}{2} \sin q_{16} \\
0 & 1 & -\frac{l_{1}}{2} \cos q_{16}
\end{array}\right] \\
& M_{3}=\left[\begin{array}{ccc}
1 & 0 & -\frac{l_{1}}{2} \sin q_{16} \\
0 & 1 & \frac{l_{1}}{2} \cos q_{16}
\end{array}\right] \\
& M_{4}=\left[\begin{array}{ll}
-l_{9} \sin q_{33} & l_{9} \cos q_{33}
\end{array}\right]
\end{aligned}
$$




$$
\begin{aligned}
& M_{5}=\left[-l_{10} \sin \left(q_{33}-86.27^{\circ}\right) \quad l_{10} \cos \left(q_{33}-86.27^{\circ}\right)\right] \\
& M_{6}=\left[\begin{array}{ccc}
1 & 0 & -\frac{l_{3}}{2} \sin q_{61} \\
0 & 1 & \frac{l_{3}}{2} \cos q_{61}
\end{array}\right] \\
& M_{7}=\left[\begin{array}{ccc}
1 & 0 & \frac{l_{3}}{2} \sin q_{61} \\
0 & 1 & -\frac{l_{3}}{2} \cos q_{61}
\end{array}\right] \\
& M_{9}=\left[\begin{array}{ccc}
1 & 0 & -\frac{l_{6}}{2} \sin q_{91} \\
0 & 1 & \frac{l_{6}}{2} \cos q_{91}
\end{array}\right] \\
& M_{10}=\left[\begin{array}{ccc}
1 & 0 & \frac{l_{6}}{2} \sin q_{91} \\
0 & 1 & -\frac{l_{6}}{2} \cos q_{91}
\end{array}\right] \\
& M_{11}=M_{12}=\left[\begin{array}{ll}
l_{8} \sin q_{110} & -l_{8} \cos q_{110}
\end{array}\right] \\
& M_{13}=\left[\begin{array}{ccc}
1 & 0 & -l_{4} \sin \left(q_{126}+83.4^{\circ}\right) \\
0 & 1 & l_{4} \cos \left(q_{126}+83.4^{\circ}\right)
\end{array}\right] \\
& M_{14}=\left[\begin{array}{ccc}
1 & 0 & -l_{5} \sin q_{126} \\
0 & 1 & l_{5} \cos q_{126}
\end{array}\right]
\end{aligned}
$$

The constant angles used in Equations A.6 and A.12 are obtained from the geometry of the two composite links. Since all the elements in the bond graph model can be mapped to typical electrical circuit elements, the entire model can be mapped to a SPICE netlist and then simulated. The initial angular positions of all the links are obtained from the kinematic analysis in Section A.1 assuming the crank starts at an angle $q_{1}=0$ (horizontal). The vertical component of the ground reaction force (GRF) is modelled as per Equation $1.5(A=200 \mathrm{~N}$ and $q=1 / 6)$. The horizontal component is approximated as a second harmonic (of amplitude $A / 7$ ) of the fundamental chosen in Equation 1.5. This GRF is applied to the bond graph model at the foot point as it traces the part of its trajectory between the landing and take-off points. Figure A.8 shows the time variation of GRF (vertical and horizontal components), reflected motor torque and link angular positions. Based on the peak value of the reflected motor torque, the torque specification of the motor is obtained. 


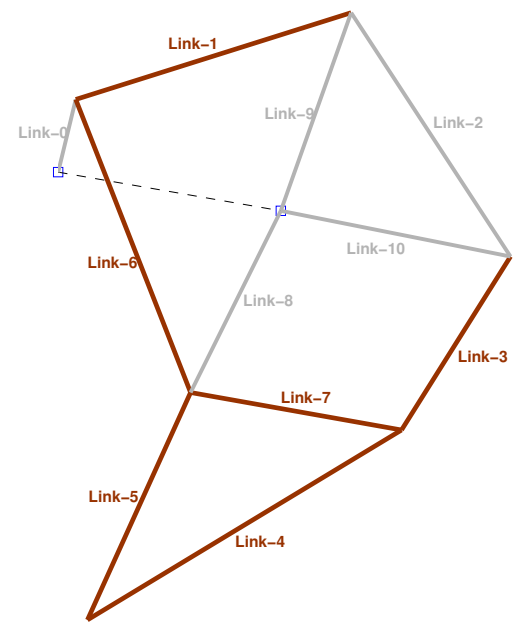

Figure A.5: Links undergoing rotation and translation.

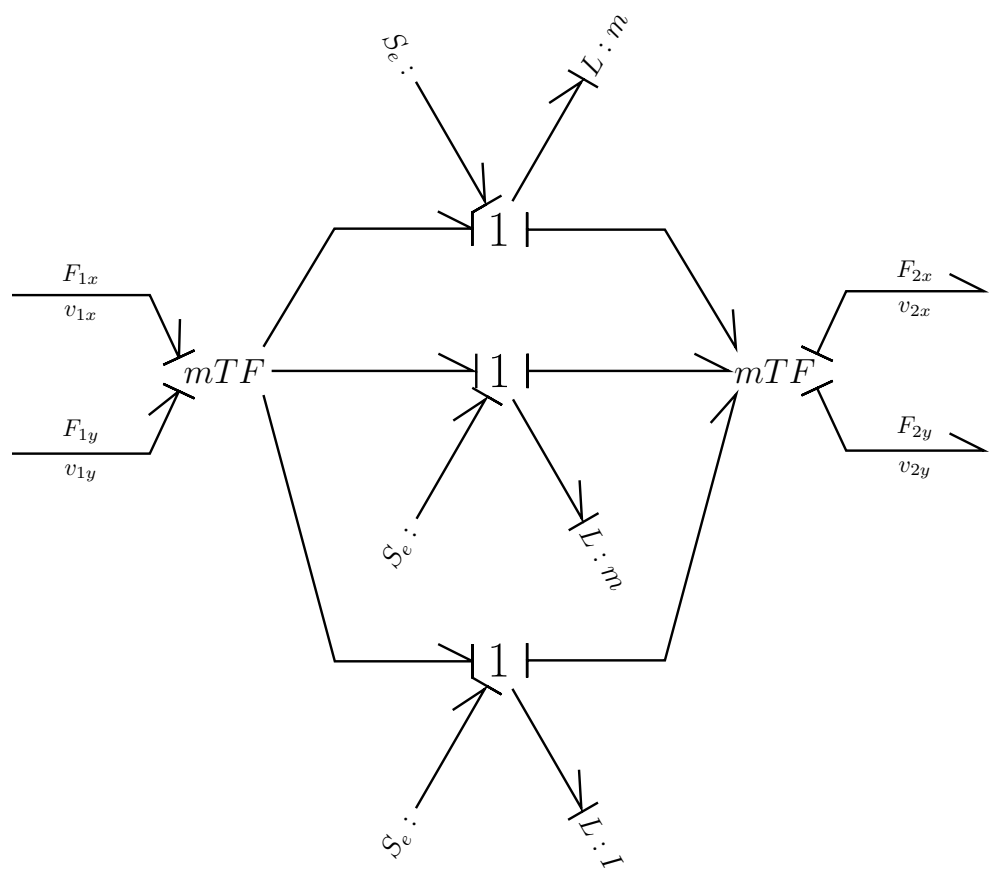

Figure A.6: Bond graph model for links undergoing rotation and translation. 


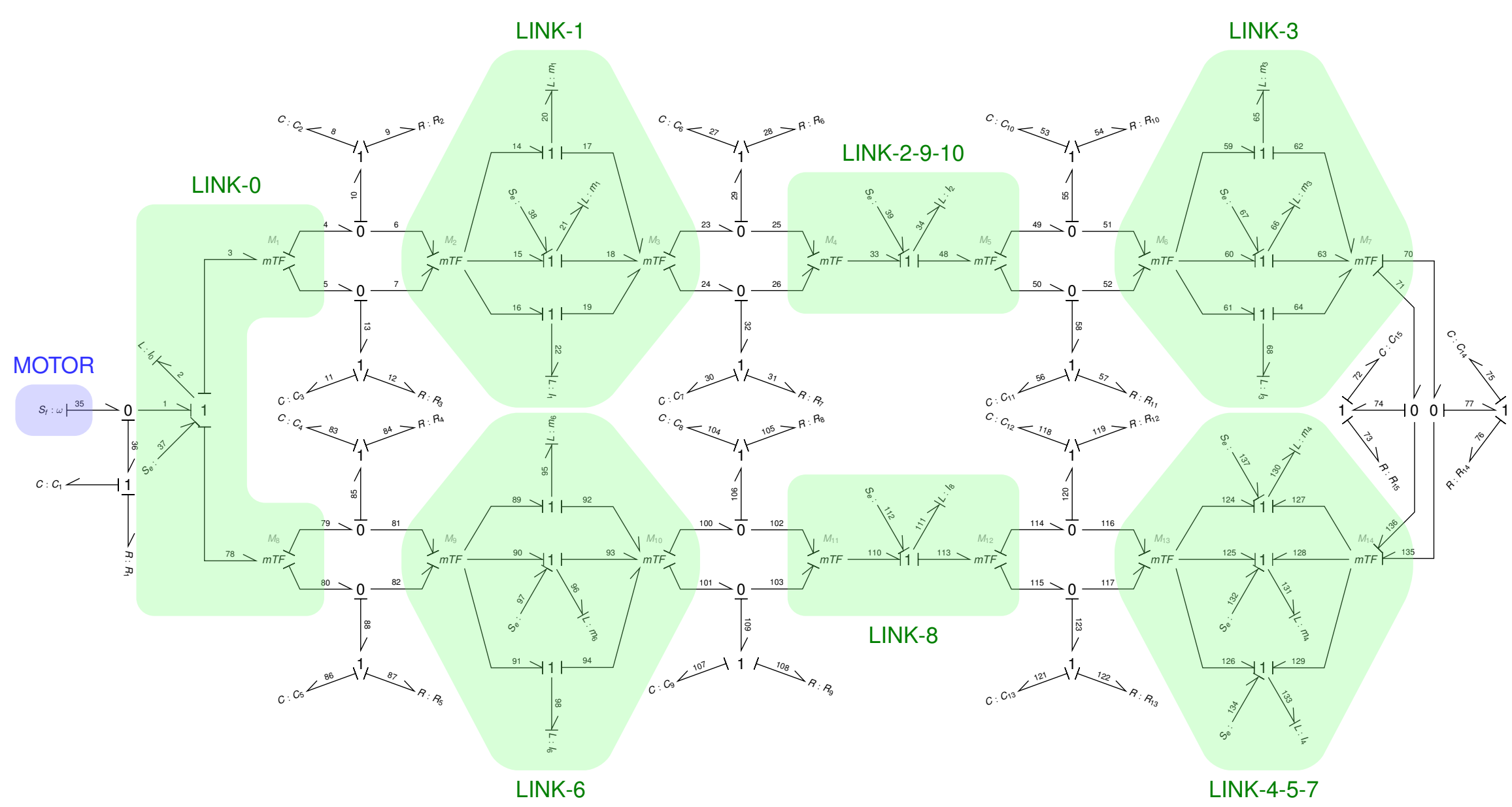

Figure A.7: Bond graph model of Jansen Mechanism 

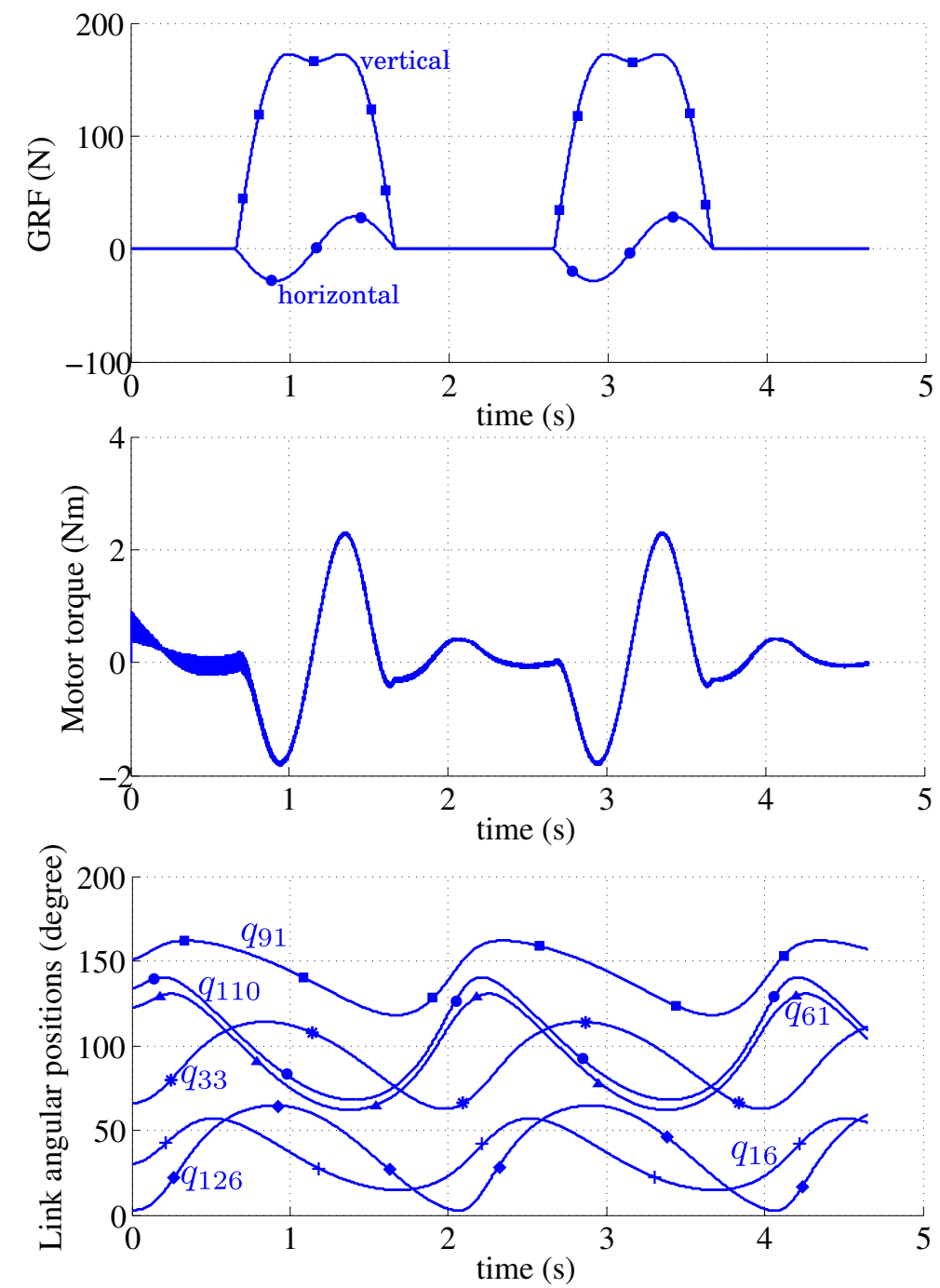

Figure A.8: Time variation of GRF (vertical and horizontal components), reflected motor torque and link angular positions for Jansen mechanism. $A=200 \mathrm{~N}, q=1 / 6$, crank length $l_{0}=0.05 \mathrm{~m}$, link density $=1 \mathrm{~kg} / \mathrm{m}$, motor angular speed $=\pi \mathrm{rad} / \mathrm{s}$. 


\section{Appendix B}

\section{CAD Details}

This appendix contains the complete CAD details for the mechanical and electrical subsystems of Chatur.

\section{B.1 Mechanical subsystem: AutoCAD drawings}

- Annular disc that couples the BLDC hub motor to the spokes

- Chatur frame: axle (main frame)

- Chatur frame: battery carrier (top piece) and axle

- Chatur frame: battery carrier (bottom piece)

- Chatur frame: battery carrier (full) and axle

- Chatur frame: tail piece

- Chatur frame: all parts

- Enclosure for electronics

- Enclosure for terminal block 


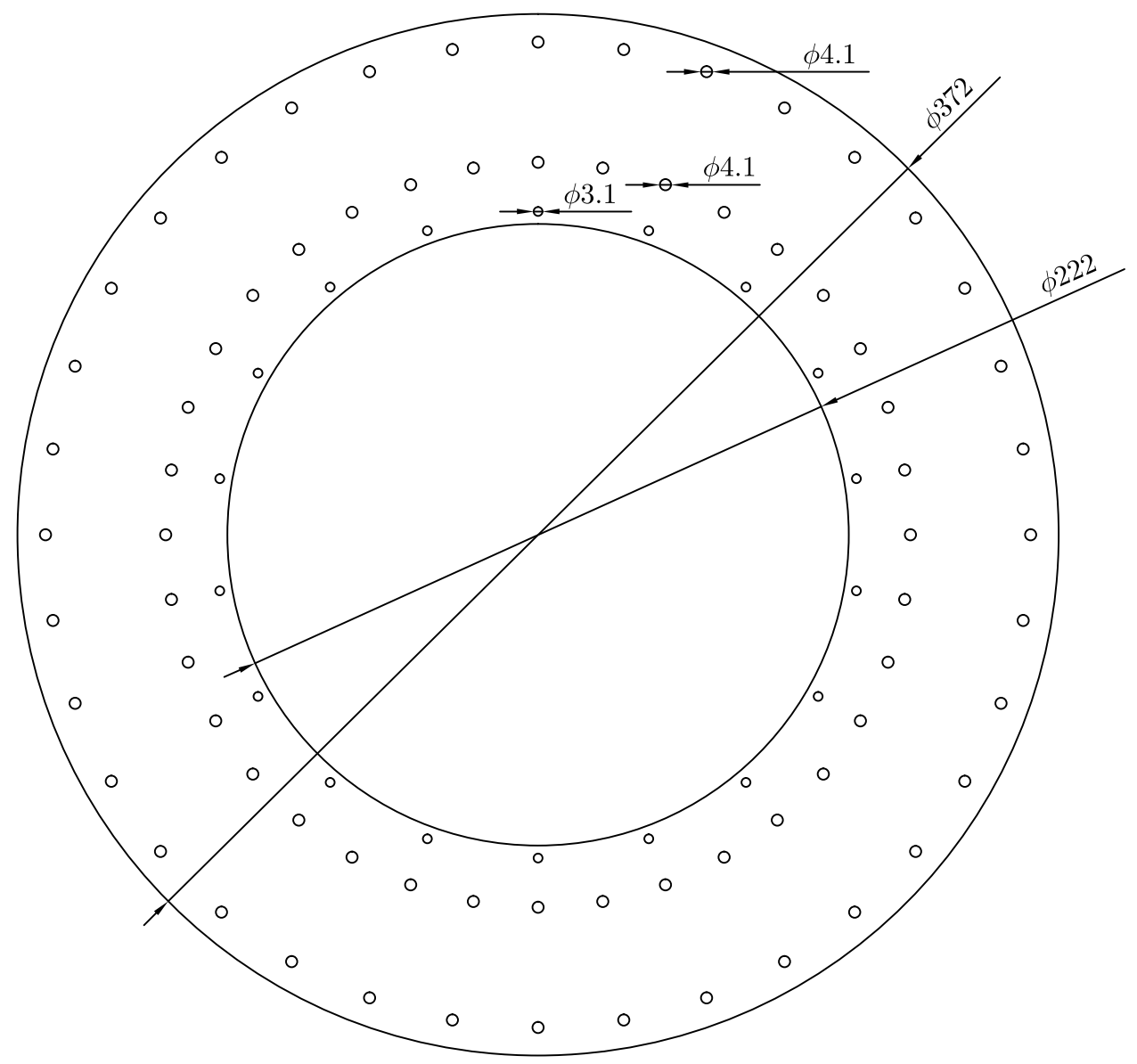

Figure B.1: CAD drawing of annular disc that couples the BLDC hub motor to the spokes (two discs per motor). It is fabricated using MS sheet of $1.2 \mathrm{~mm}$ thickness. All dimensions are in $\mathrm{mm}$. 


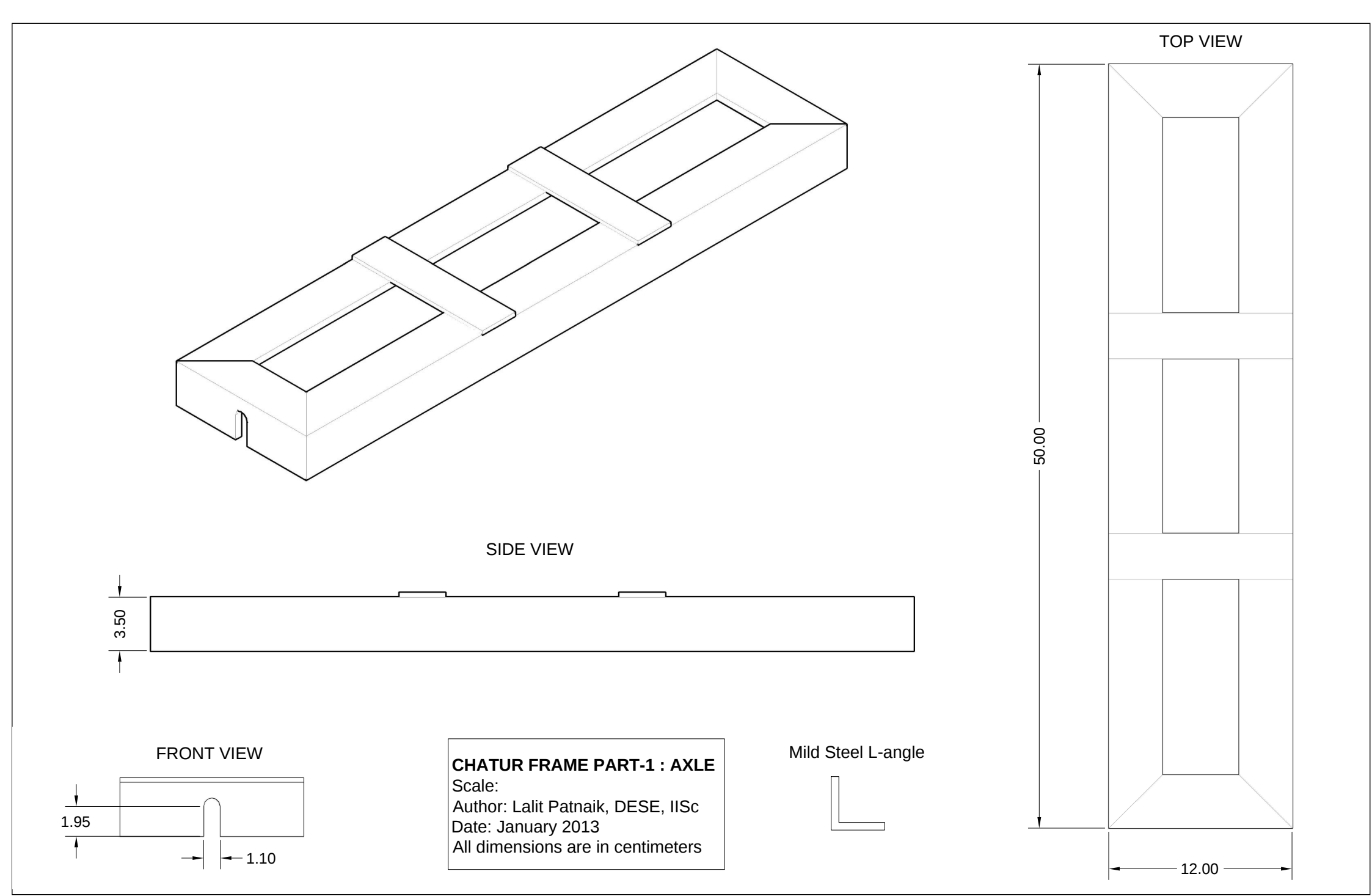

Figure B.2: CAD drawing of Chatur frame: axle (main frame). 


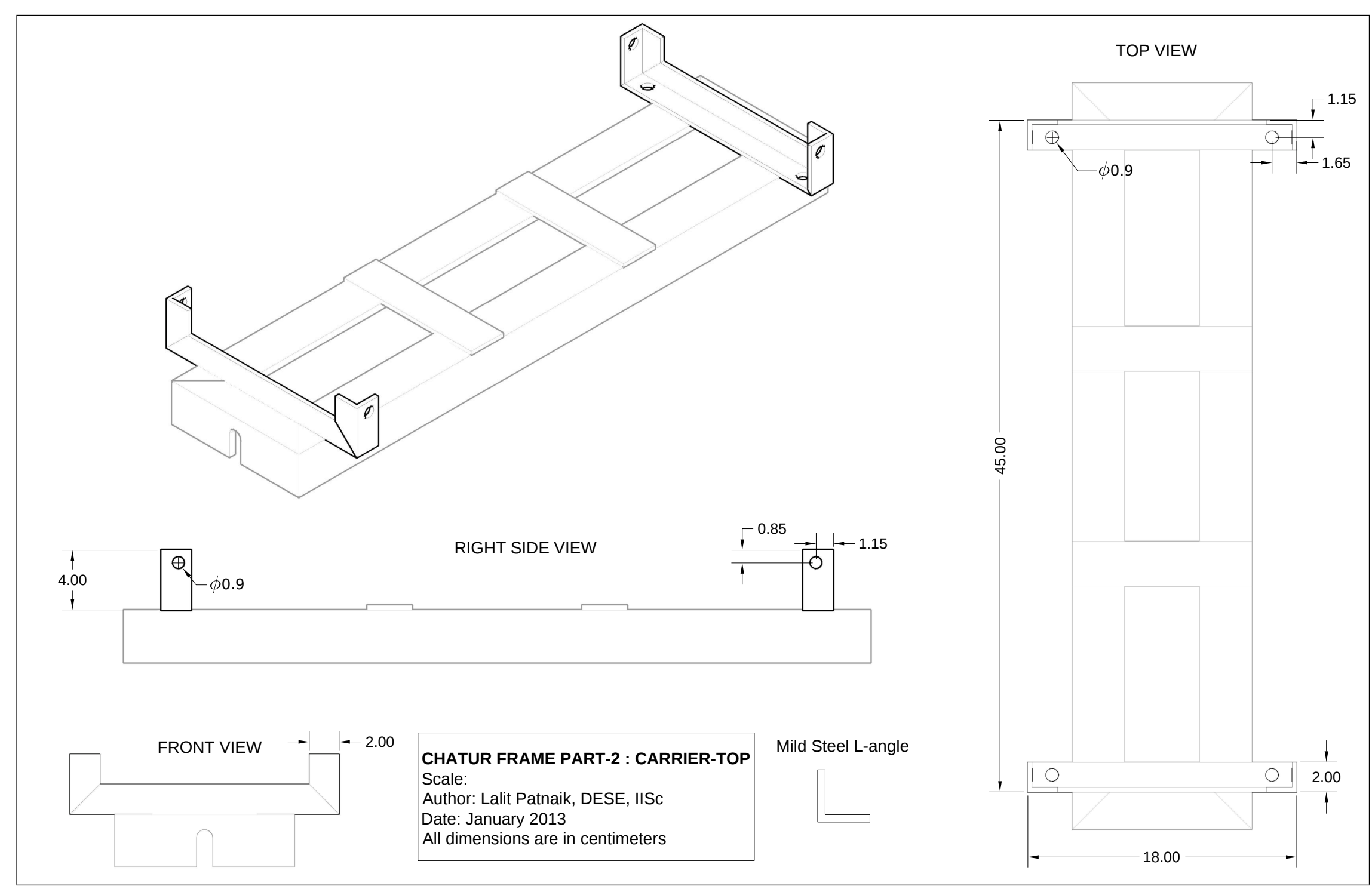

Figure B.3: CAD drawing of Chatur frame: battery carrier (top piece) and axle. 


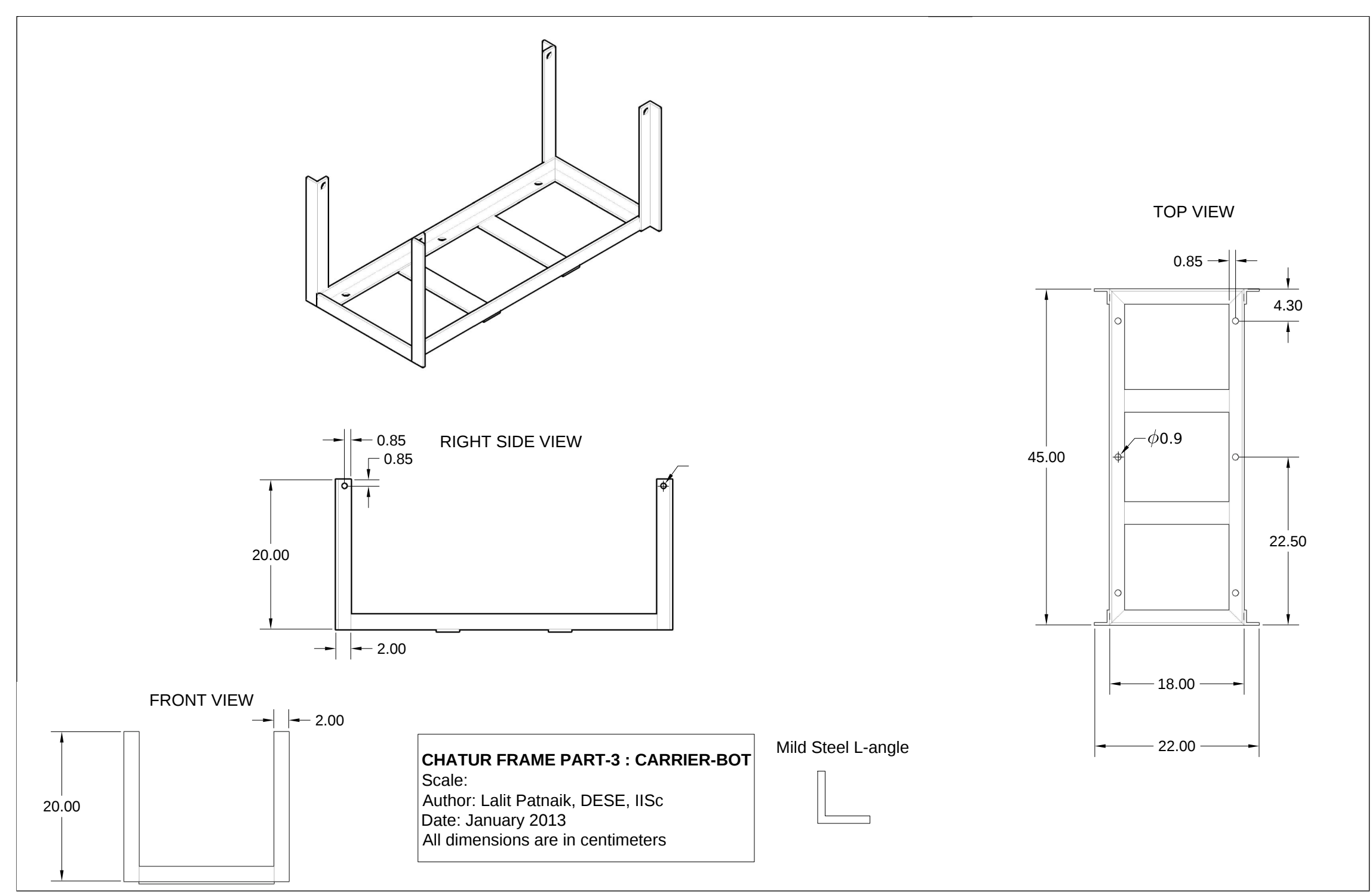




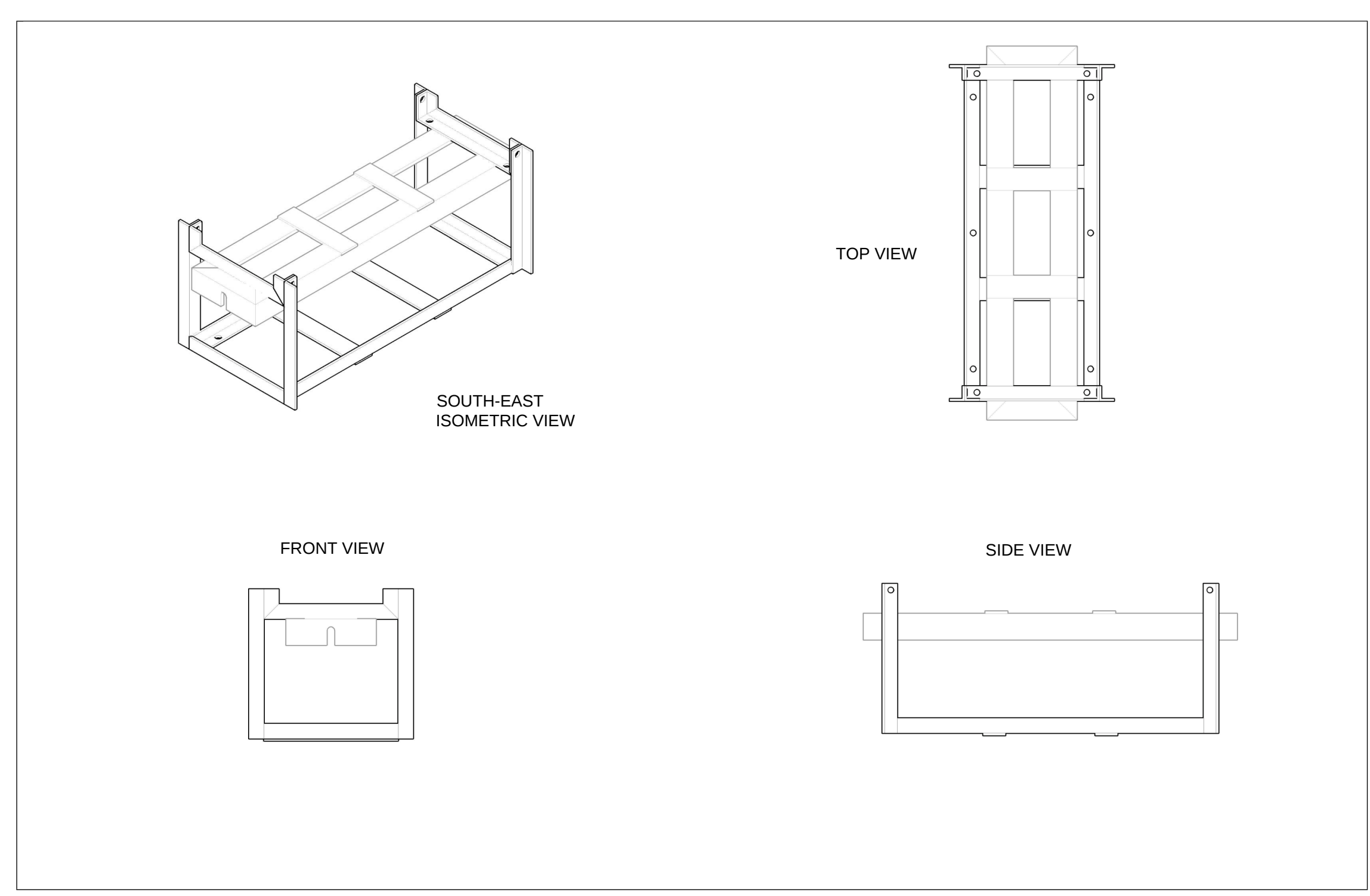

Figure B.5: CAD drawing of Chatur frame: battery carrier (full) and axle. 


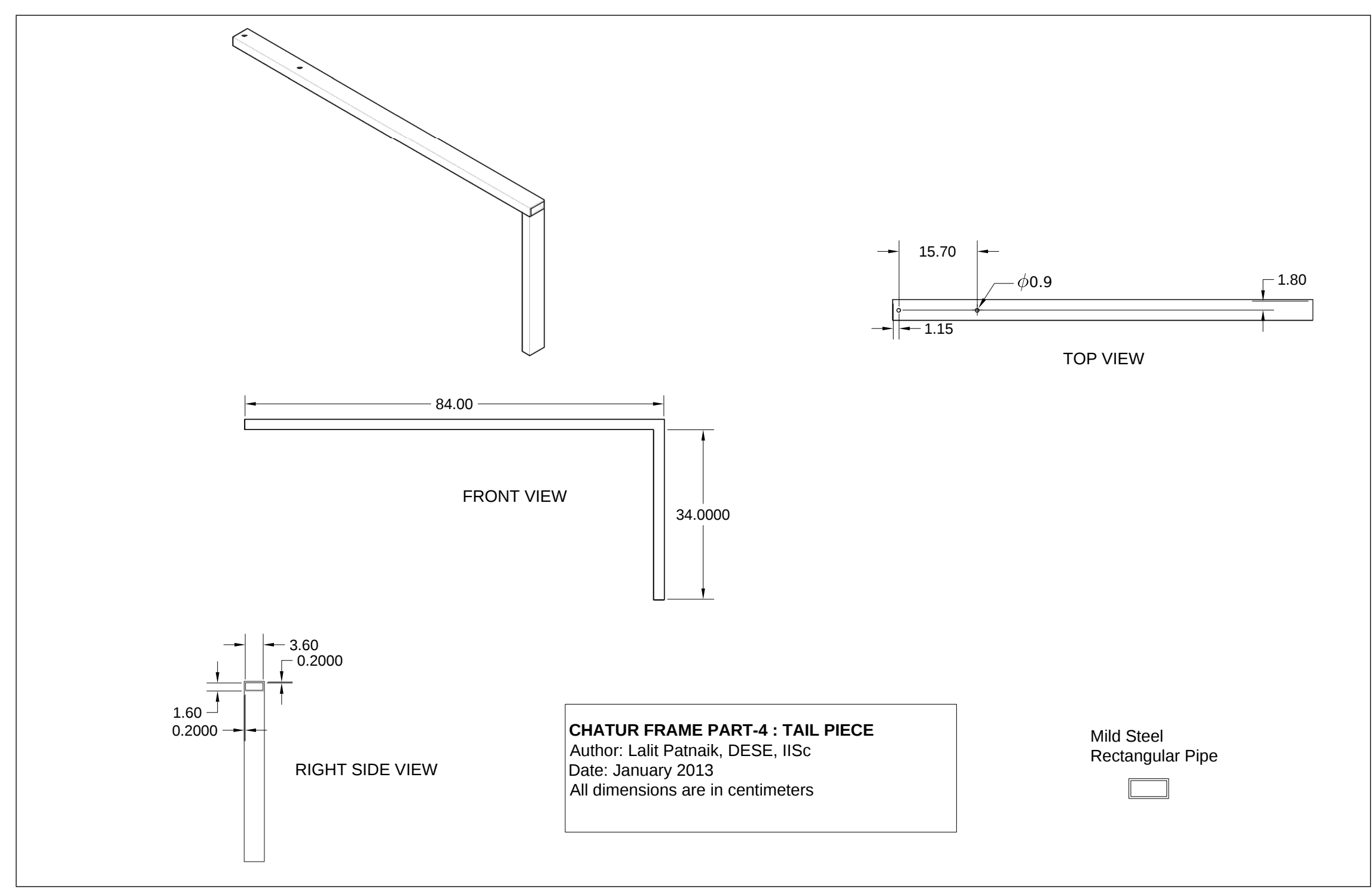

Figure B.6: CAD drawing of Chatur frame: tail piece. Castor wheel (not shown) is attached at the bottom as shown in Figure $4.2 \mathrm{~b}$. 


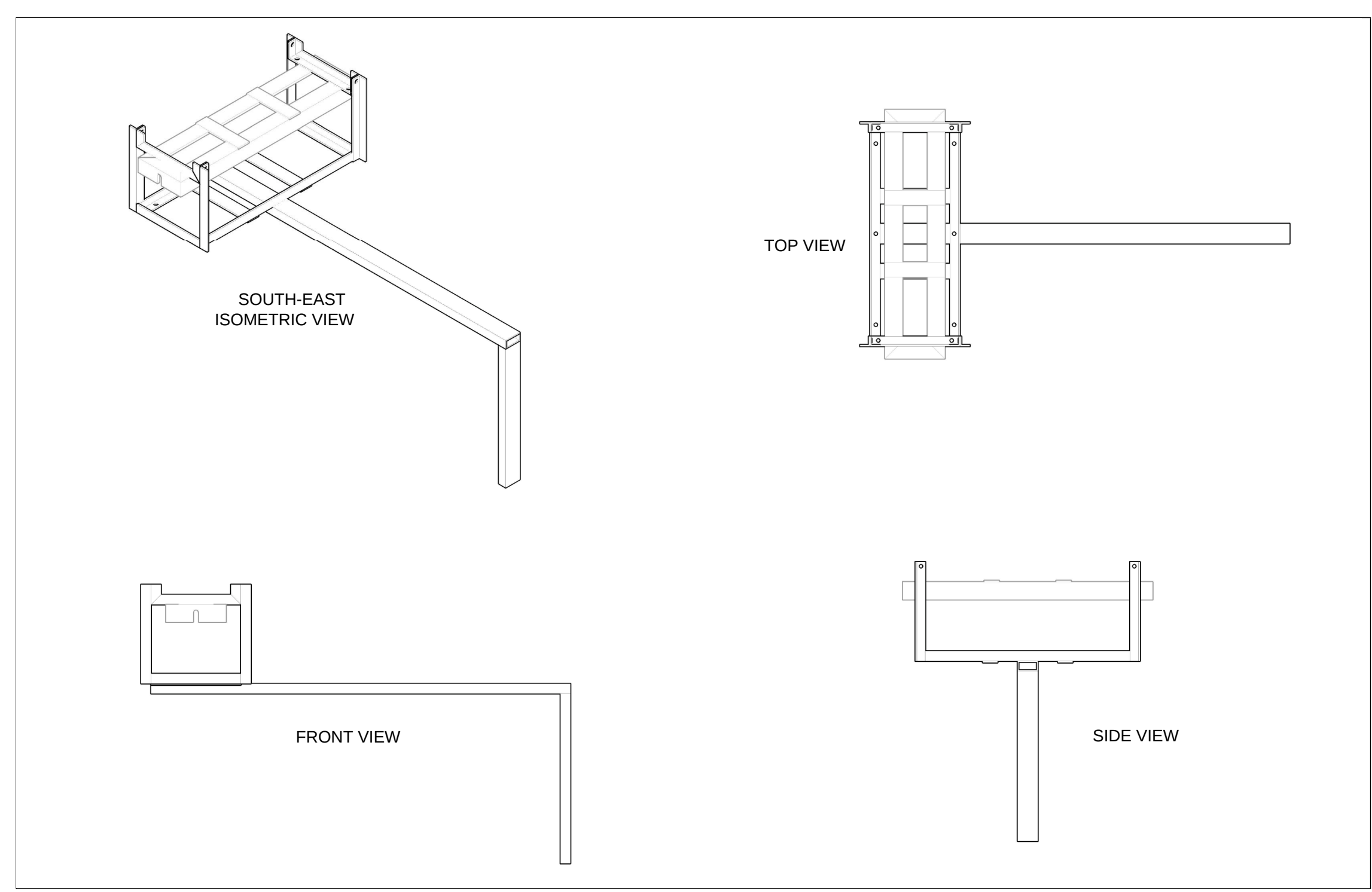

Figure B.7: CAD drawing of Chatur frame: all parts. Assembly is done using M7 nuts and bolts. 


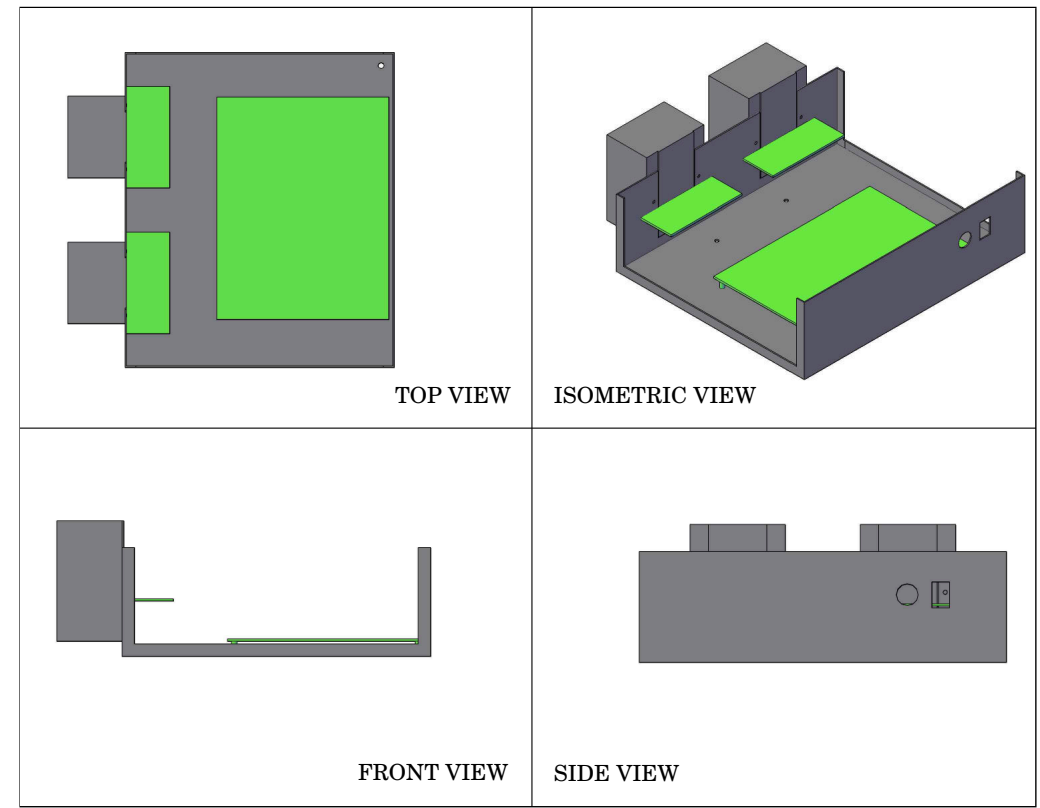

Figure B.8: CAD rendering of enclosure for electronics. The small green rectangles are the CIPOS inverter boards. The large green rectangle is the Chatur controller board. The blocks at the wall of the enclosure are the heat sinks. The slots on the opposite wall are for mounting the power switch and fuse for the controller board.

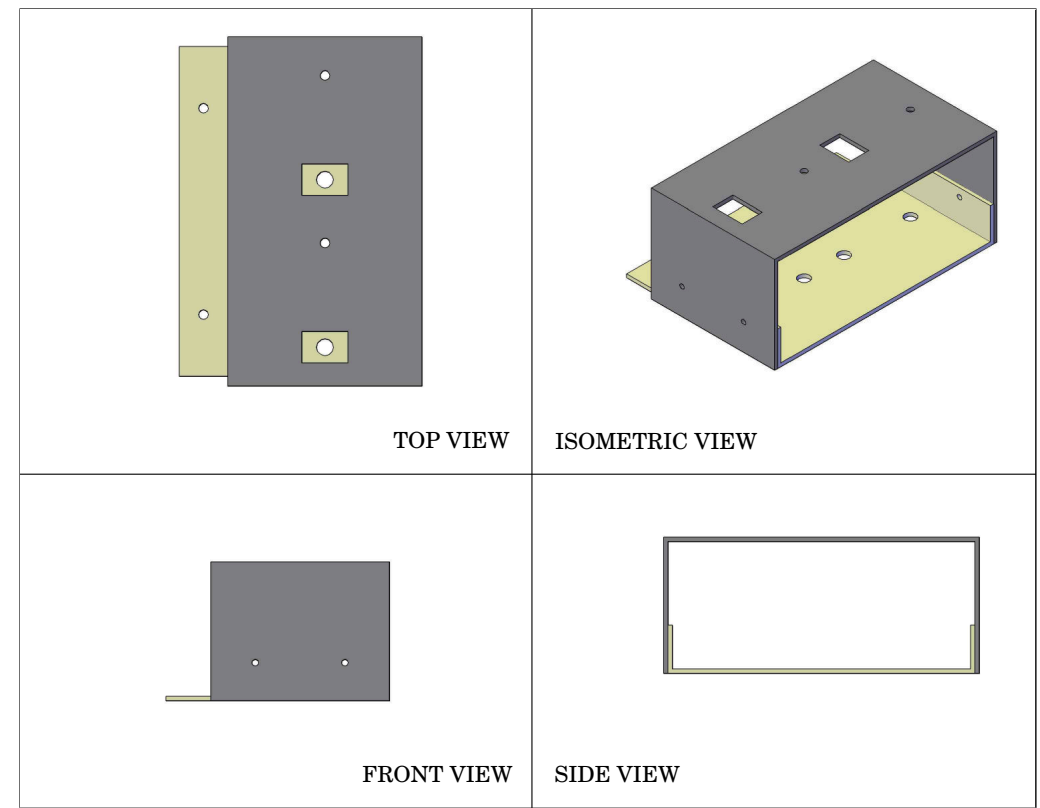

Figure B.9: CAD rendering of enclosure for terminal blocks. The terminal blocks are not shown in the figure. The enclosure has two open sides. The wires from the battery bank come in on one side. The wires to the inverters go out on the other side. The slots on the roof are for mounting the power switches and fuses for the inverter board. 


\section{B.2 Electrical subsystem}

- PCB Schematics

- OrCAD schematics of CIPOS ${ }^{T M}$ inverter board

- gEDA schematics of Chatur board

* Top level block diagram (top.sch)

* PSoC ${ }^{\circledR} 5$ and its peripherals (psoc.sch)

* Opto-isolation of PWM and fault signals (opto.sch)

* Sensing and signal conditioning (sense.sch)

* Three output flyback converter (power.sch)

- MATLAB/octave script for design of three-output flyback transformer used on Chatur board (flyback.m)

- PSoC ${ }^{\circledR}$ port map as applicable on Chatur board

- Top level PSoC ${ }^{\circledR}$ schematics

- Page-1: Fault servicing, position sensing and PWM generation for motor-1

- Page-2: ADCs and UART

- Page-3: Fault servicing, position sensing and PWM generation for motor-2

- Page-4: Timers and IDACs

- C-code for the control algorithm (embedded software that runs on the ARM core) 


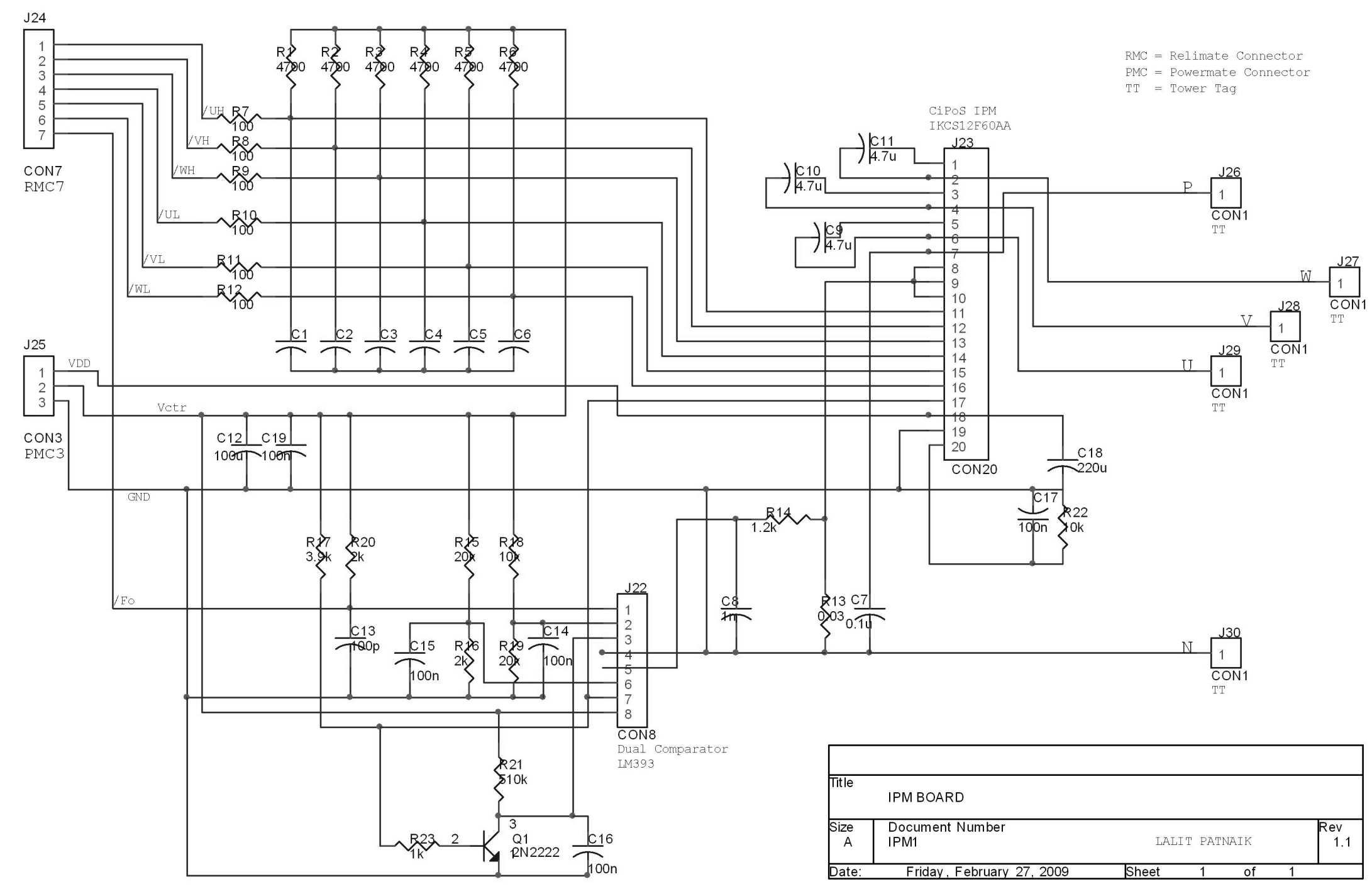

Figure B.10: CIPOS $^{\top \mathrm{T}}$ inverter board OrCAD schematics 


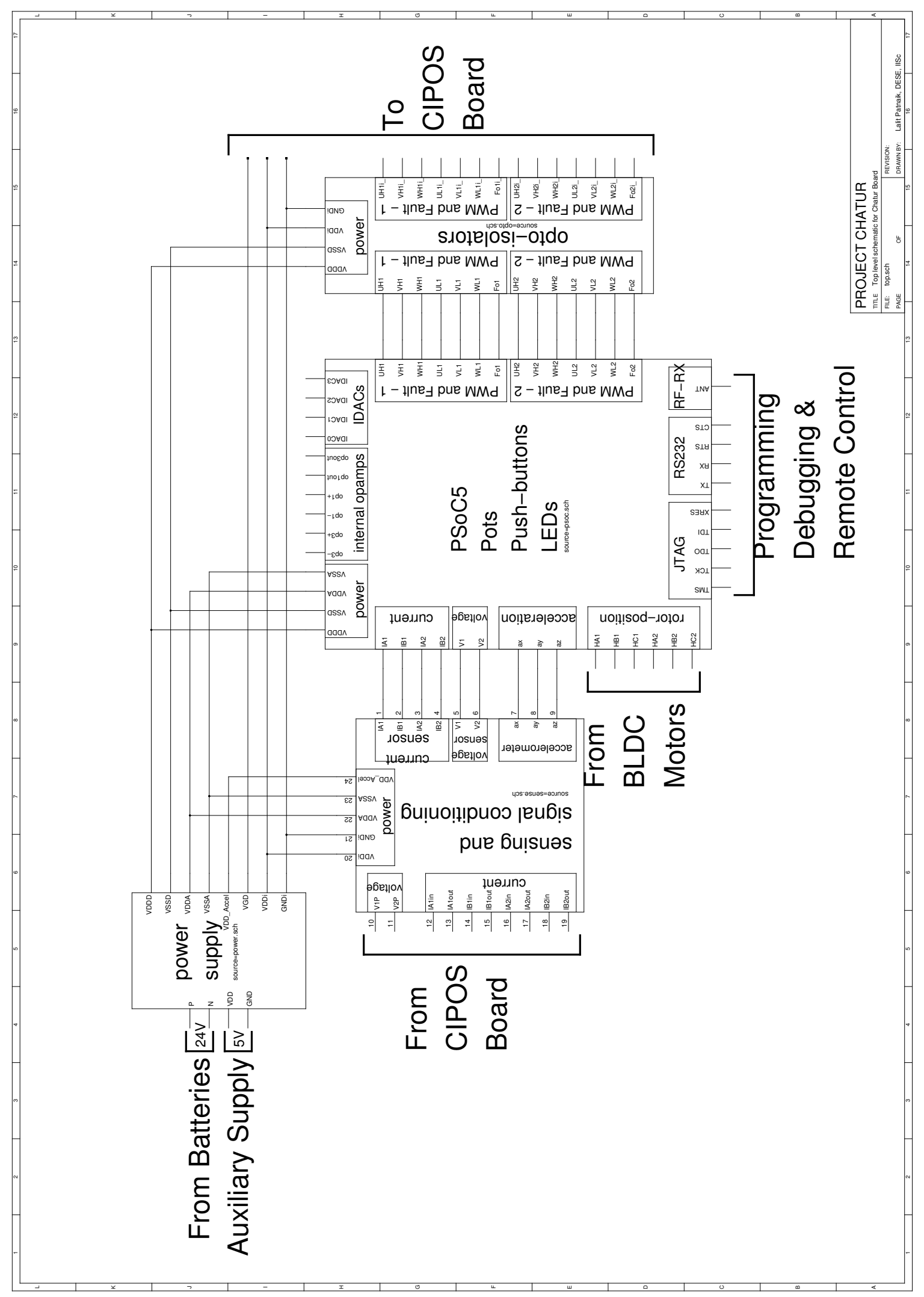

Figure B.11: gEDA schematics of Chatur board: Top level block diagram 


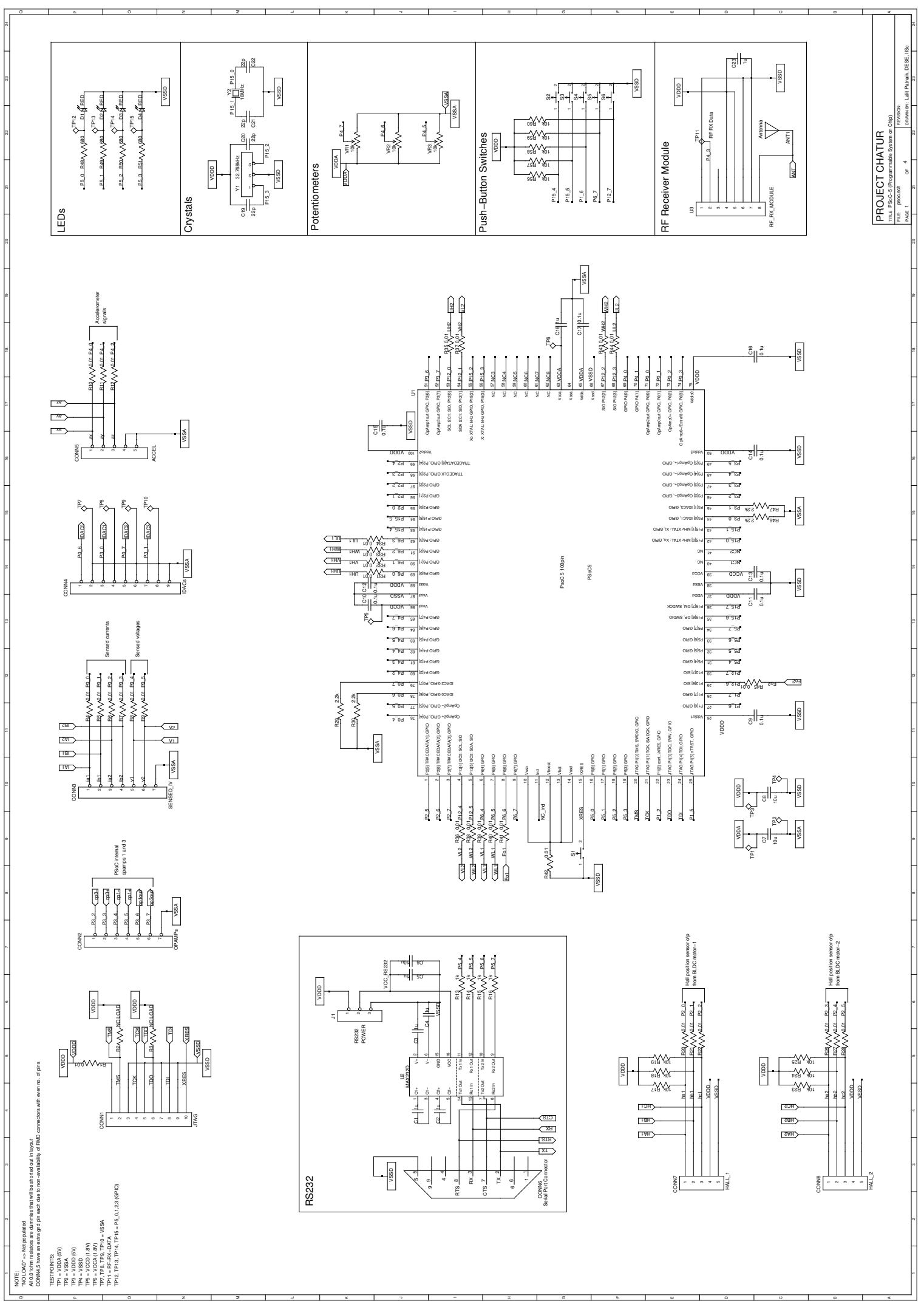

Figure B.12: gEDA schematics of Chatur board: $\mathrm{PSoC}^{\circledR} 5$ and its peripherals 


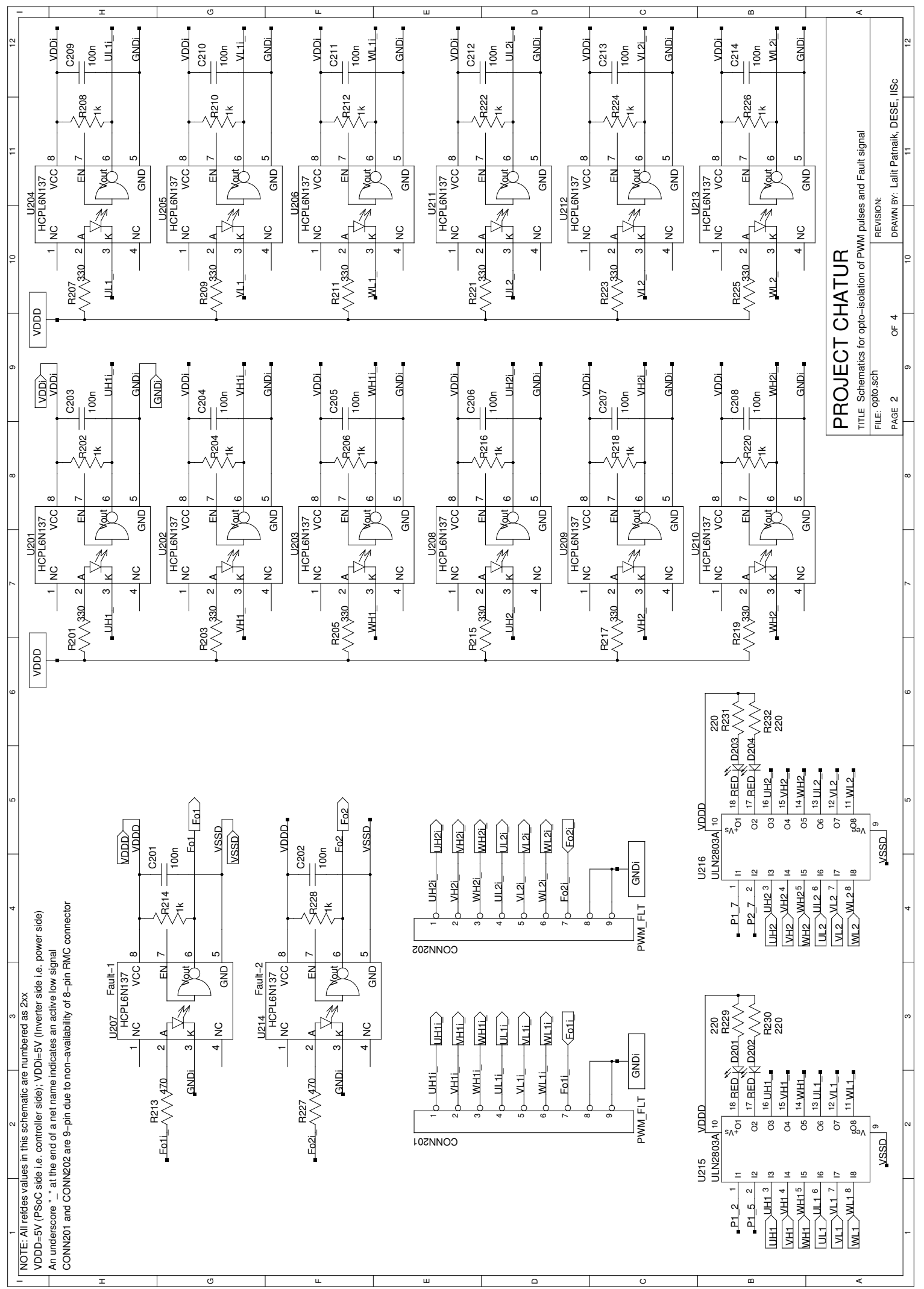

Figure B.13: gEDA schematics of Chatur board: Opto-isolation of PWM and fault signals 


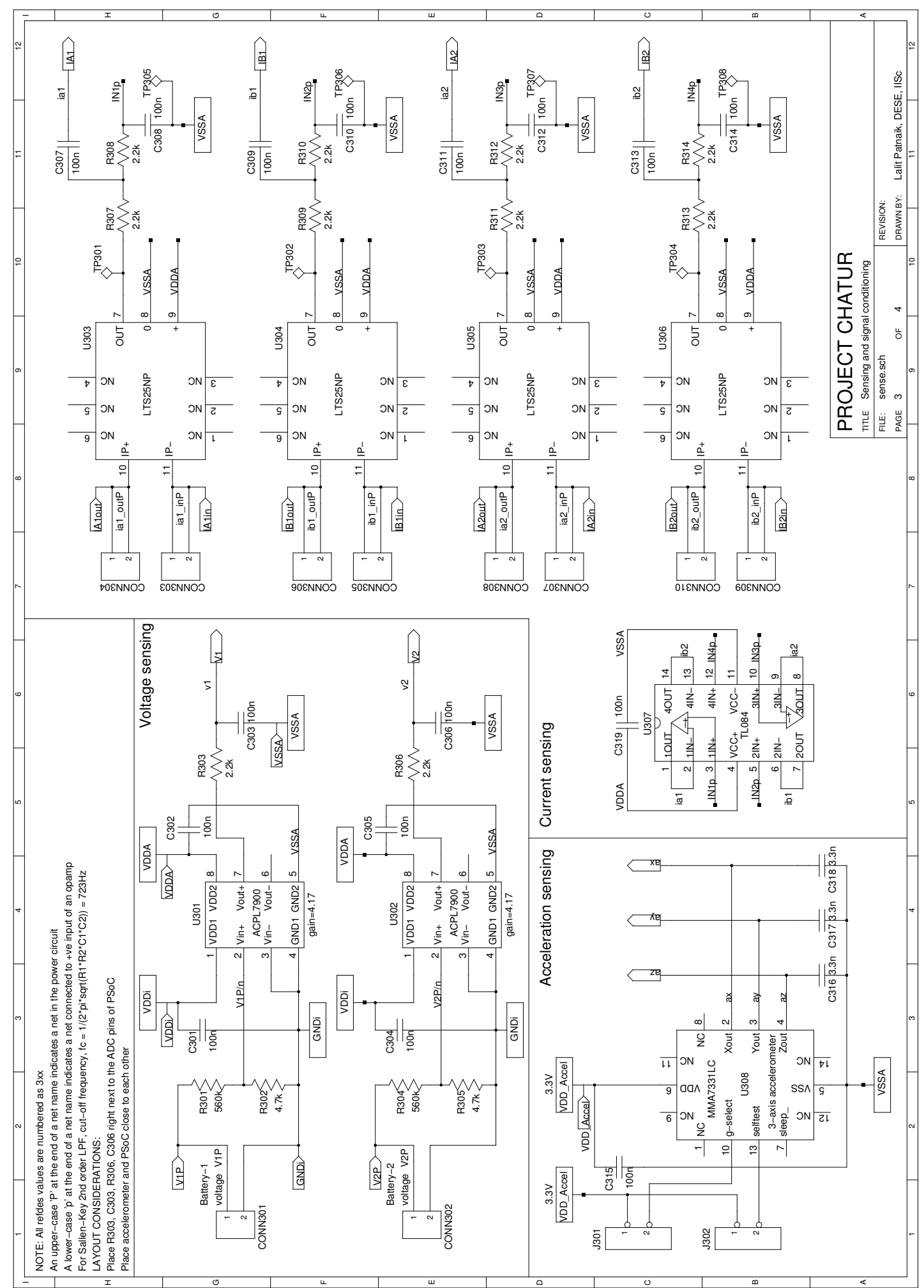

Figure B.14: gEDA schematics of Chatur board: Sensing and signal conditioning 


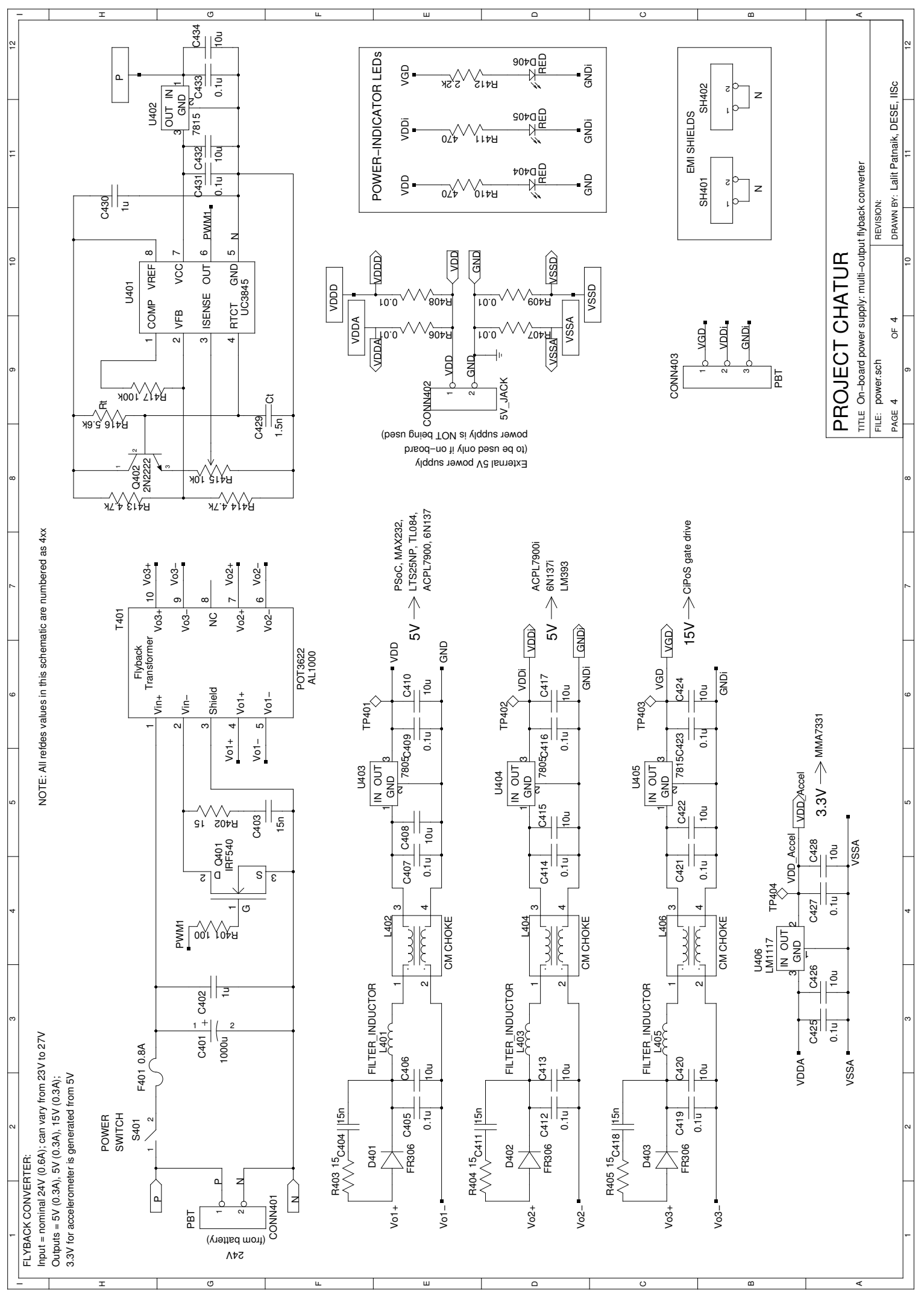

Figure B.15: gEDA schematics of Chatur board: Three output flyback converter 
$1 \%$ Flyback transformer (multi-winding inductor) design

\% Author: Lalit Patnaik, DESE, IISc

$\%$

\% Ref: "Power Electronics - Essentials and Applications"

$\%$ by L Umanand, pp. $379-383$

Vin_min $=23$

Vin_max $=27$

Dmin $=0.25$

Dmax $=0.5$

$11 \mathrm{fs}=100 \mathrm{e} 3$

$\mathrm{Ip}=0.6 \%(10 \mathrm{~V} * 0.3 \mathrm{~A}+10 \mathrm{~V} * 0.3 \mathrm{~A}+20 \mathrm{~V} * 0.3 \mathrm{~A}) / 23 \mathrm{~V}$

del_Ip $=0.1$

$\mathrm{Lp}=$ Vin_max $* 0.4 /\left(\mathrm{del}_{\mathrm{L}} \mathrm{I} \mathrm{p} * \mathrm{fs}\right)$

Ip_max $=I_{p}+$ del_Ip $/ 2$

$16 \quad \mathrm{E}_{-} \mathrm{L}=0.5 * \mathrm{Lp} * \mathrm{Ip} \_\mathrm{max}^{\wedge} 2$

$\mathrm{Kw}=0.3$

$\mathrm{Kc}=\operatorname{sqrt}(\mathrm{Dmin})$

$\mathrm{J}=4 \mathrm{e} 6 \quad \% \quad 4 \quad \mathrm{~A} / \mathrm{mm} 2$

$\mathrm{Bm}=0.25$

$21 \mathrm{Ap}=2 * \mathrm{E} \_\mathrm{L} /(\mathrm{Kw} * \mathrm{Kc} * \mathrm{~J} * \mathrm{Bm})$

$\%$ Choose core with higher Ap than that obtained above

$\%$ and get the core specifications

$26 \%$ For $36 / 22$ POT core:

$\mathrm{AL}=1000 \mathrm{e}-9 \% \mathrm{AL}$ is generally specified in $\mathrm{nH} / \mathrm{turns}^{\wedge} 2$

Aw $=0.75 \mathrm{e}-4$

$\mathrm{Np}=\operatorname{ceil}(\operatorname{sqrt}(\mathrm{Lp} / \mathrm{AL}))$

31

$\mathrm{ap}=\mathrm{Ip} / \mathrm{J}$

Is $1=0.3$

Is $2=0.3$

Is $3=0.3 \%$ average

as $1=\mathrm{Is} 1 / \mathrm{J}$

36

as $2=\mathrm{Is} 2 / \mathrm{J}$

as $3=$ Is $3 / \mathrm{J}$

\% Enter standard wire-guage cross-section areas:

$\%$ SWG-22 $=0.397 \mathrm{e}-6 \quad ;$ SWG-24 $=0.2454 \mathrm{e}-6 ;$ SWG-26 $=0.1642 \mathrm{e}-6 ;$

$41 \%$ SWG-28 $=0.111 \mathrm{e}-6 ;$ SWG $-30=0.0779 \mathrm{e}-6$

ap $=0.1642 \mathrm{e}-6 \%$ SWG-26

as $1=0.1642 \mathrm{e}-6 \%$ SWG-26

as $2=0.1642 \mathrm{e}-6 \%$ SWG -26

as $3=0.1642 \mathrm{e}-6 \%$ SWG -26

46

avl_Warea $=\mathrm{Aw} * \mathrm{Kw}$

reqd_Warea $=$ ap $* \mathrm{~Np}+$ as $1 * \mathrm{~Np}+$ as $2 * \mathrm{~Np}+$ as $3 * 2 * \mathrm{~Np}$

Listing B.1: MATLAB/octave script for design of three-output flyback transformer. 
Table B.1: PSoC ${ }^{\circledR}$ port map as applicable on Chatur board.

\begin{tabular}{|c|c|c|c|}
\hline PSoC ${ }^{\circledR}$ port & Signal & Description & Available at \\
\hline $\mathrm{P} 0[0]$ & Ia1 & Sensed current & CONN3 \\
\hline $\mathrm{P} 0[1]$ & Ib1 & Sensed current & CONN3 \\
\hline $\mathrm{P} 0[2]$ & Ia2 & Sensed current & CONN3 \\
\hline $\mathrm{P} 0[3]$ & $\mathrm{Ib} 2$ & Sensed current & CONN3 \\
\hline $\mathrm{P} 0[4]$ & Vdc1 & Sensed voltage & CONN3 \\
\hline $\mathrm{P} 0[5]$ & Vdc2 & Sensed voltage & CONN3 \\
\hline $\mathrm{P} 0[6]$ & IDAC0 & DAC output & CONN4 \\
\hline $\mathrm{P} 0[7]$ & IDAC2 & DAC output & CONN4 \\
\hline $\mathrm{P} 1[0]$ & JTAG-TMS & JTAG & CONN1 \\
\hline $\mathrm{P} 1[1]$ & JTAG-TCK & JTAG & CONN1 \\
\hline $\mathrm{P} 1[2]$ & CHAN & SAR ADC channel select & D201 (Green LED) \\
\hline $\mathrm{P} 1[3]$ & JTAG-TDO & JTAG & CONN1 \\
\hline $\mathrm{P} 1[4]$ & JTAG-TDI & JTAG & CONN1 \\
\hline $\mathrm{P} 1[5]$ & $\mathrm{SOC}$ & SAR ADC SOC & D202 (Green LED) \\
\hline $\mathrm{P} 1[6]$ & - & Push-button switch & $\mathrm{S} 4$ \\
\hline $\mathrm{P} 1[7]$ & RF_FLT & RF fault & D203 (Green LED) \\
\hline $\mathrm{P} 2[0]$ & ha1 & Motor-1 hall sensor-a & CONN7 \\
\hline $\mathrm{P} 2[1]$ & hb1 & Motor-1 hall sensor-b & CONN7 \\
\hline $\mathrm{P} 2[2]$ & hc1 & Motor-1 hall sensor-c & CONN7 \\
\hline $\mathrm{P} 2[3]$ & ha2 & Motor-2 hall sensor-a & CONN8 \\
\hline $\mathrm{P} 2[4]$ & hb2 & Motor-2 hall sensor-b & CONN8 \\
\hline $\mathrm{P} 2[5]$ & hc2 & Motor-2 hall sensor-c & CONN8 \\
\hline $\mathrm{P} 2[6]$ & - & - & - \\
\hline $\mathrm{P} 2[7]$ & - & - & D204 (Green LED) \\
\hline $\mathrm{P} 3[0]$ & IDAC1 & DAC output & CONN4 \\
\hline P3[1] & IDAC3 & DAC output & CONN4 \\
\hline $\mathrm{P} 3[2]$ & - & - & - \\
\hline $\mathrm{P} 3[3]$ & - & - & - \\
\hline $\mathrm{P} 3[4]$ & - & - & - \\
\hline P3[5] & - & - & - \\
\hline
\end{tabular}


Table B.1 - Continued

\begin{tabular}{|c|c|c|c|}
\hline PSoC $^{\circledR}$ port & Signal & Description & Available at \\
\hline P3[6] & - & - & - \\
\hline $\mathrm{P} 3[7]$ & - & - & - \\
\hline $\mathrm{P} 4[0]$ & $a x$ & Accelerometer output & CONN5 \\
\hline $\mathrm{P} 4[1]$ & ay & Accelerometer output & CONN5 \\
\hline $\mathrm{P} 4[2]$ & $\mathrm{az}$ & Accelerometer output & CONN5 \\
\hline $\mathrm{P} 4[3]$ & RF_RX & $\mathrm{RF}$ receiver data & TP11 \\
\hline $\mathrm{P} 4[4]$ & - & - & - \\
\hline $\mathrm{P} 4[5]$ & - & Potentiometer & VR3 \\
\hline $\mathrm{P} 4[6]$ & - & Potentiometer & VR2 \\
\hline $\mathrm{P} 4[7]$ & - & Potentiometer & VR1 \\
\hline $\mathrm{P} 5[0]$ & FLT_INV1 & Fault in Inverter-1 & D1 (Red LED) \\
\hline $\mathrm{P} 5[1]$ & FLT_INV1 & Fault in Inverter-2 & D2 (Red LED) \\
\hline $\mathrm{P} 5[2]$ & TORQ_ON & Motor energized & D3 (Red LED) \\
\hline $\mathrm{P} 5[3]$ & WAIT & In WAIT state & D4 (Red LED) \\
\hline $\mathrm{P} 5[4]$ & - & RS232 & CONN6 \\
\hline $\mathrm{P} 5[5]$ & - & RS232 & CONN6 \\
\hline $\mathrm{P} 5[6]$ & - & RS232 & CONN6 \\
\hline $\mathrm{P} 5[7]$ & - & $\mathrm{RS} 232$ & CONN6 \\
\hline $\mathrm{P} 6[0]$ & UH1i & Inverter-1 PWM & CONN201 \\
\hline $\mathrm{P} 6[1]$ & VH1i & Inverter-1 PWM & CONN201 \\
\hline $\mathrm{P} 6[2]$ & WH1i_ & Inverter-1 PWM & CONN201 \\
\hline $\mathrm{P} 6[3]$ & UL1i_ & Inverter-1 PWM & CONN201 \\
\hline $\mathrm{P} 6[4]$ & VL1i & Inverter-1 PWM & CONN201 \\
\hline $\mathrm{P} 6[5]$ & WL1i & Inverter-1 PWM & CONN201 \\
\hline $\mathrm{P} 6[6]$ & $\mathrm{Foli}_{-}$ & Inverter-1 Fault & CONN201 \\
\hline $\mathrm{P} 6[7]$ & - & Push-button switch & $\mathrm{S} 5$ \\
\hline $\mathrm{P} 12[0]$ & UH2i_ & Inverter-2 PWM & CONN202 \\
\hline $\mathrm{P} 12[1]$ & VH2i_ & Inverter-2 PWM & CONN202 \\
\hline $\mathrm{P} 12[2]$ & WH2i_ & Inverter-2 PWM & CONN202 \\
\hline $\mathrm{P} 12[3]$ & UL2i & Inverter-2 PWM & CONN202 \\
\hline
\end{tabular}


Table B.1 - Continued

\begin{tabular}{|c|c|c|c|}
\hline PSoC $^{\circledR}$ port & Signal & Description & Available at \\
\hline $\mathrm{P} 12[4]$ & VL2i & Inverter-2 PWM & CONN202 \\
\hline $\mathrm{P} 12[5]$ & WL2i & Inverter-2 PWM & CONN202 \\
\hline $\mathrm{P} 12[6]$ & Fo2i & Inverter-2 Fault & CONN202 \\
\hline $\mathrm{P} 12[7]$ & - & Push-button switch & $\mathrm{S} 6$ \\
\hline $\mathrm{P} 15[0]$ & - & Crystal & XTAL-Y2 \\
\hline $\mathrm{P} 15[1]$ & - & Crystal & XTAL-Y2 \\
\hline $\mathrm{P} 15[2]$ & - & Crystal & XTAL-Y1 \\
\hline $\mathrm{P} 15[3]$ & - & Crystal & XTAL-Y1 \\
\hline $\mathrm{P} 15[4]$ & - & Push-button switch & $\mathrm{S} 2$ \\
\hline $\mathrm{P} 15[5]$ & - & Push-button switch & $\mathrm{S} 3$ \\
\hline $\mathrm{P} 15[6]$ & - & - & - \\
\hline $\mathrm{P} 15[7]$ & - & - & - \\
\hline
\end{tabular}




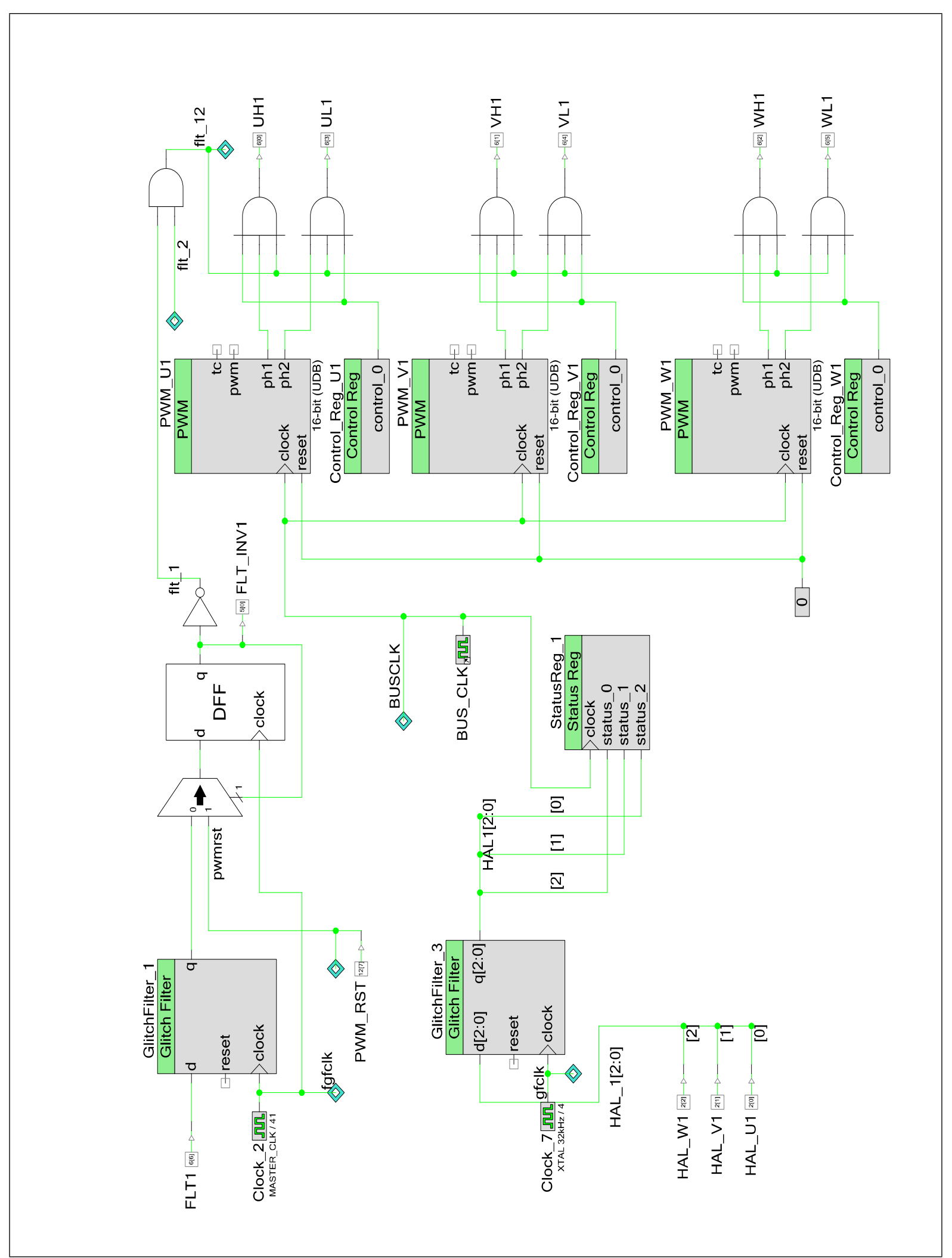

Figure B.16: PSoC $^{\circledR}$ top-level schematics (Page-1): Fault servicing, position sensing and PWM generation for motor-1. 


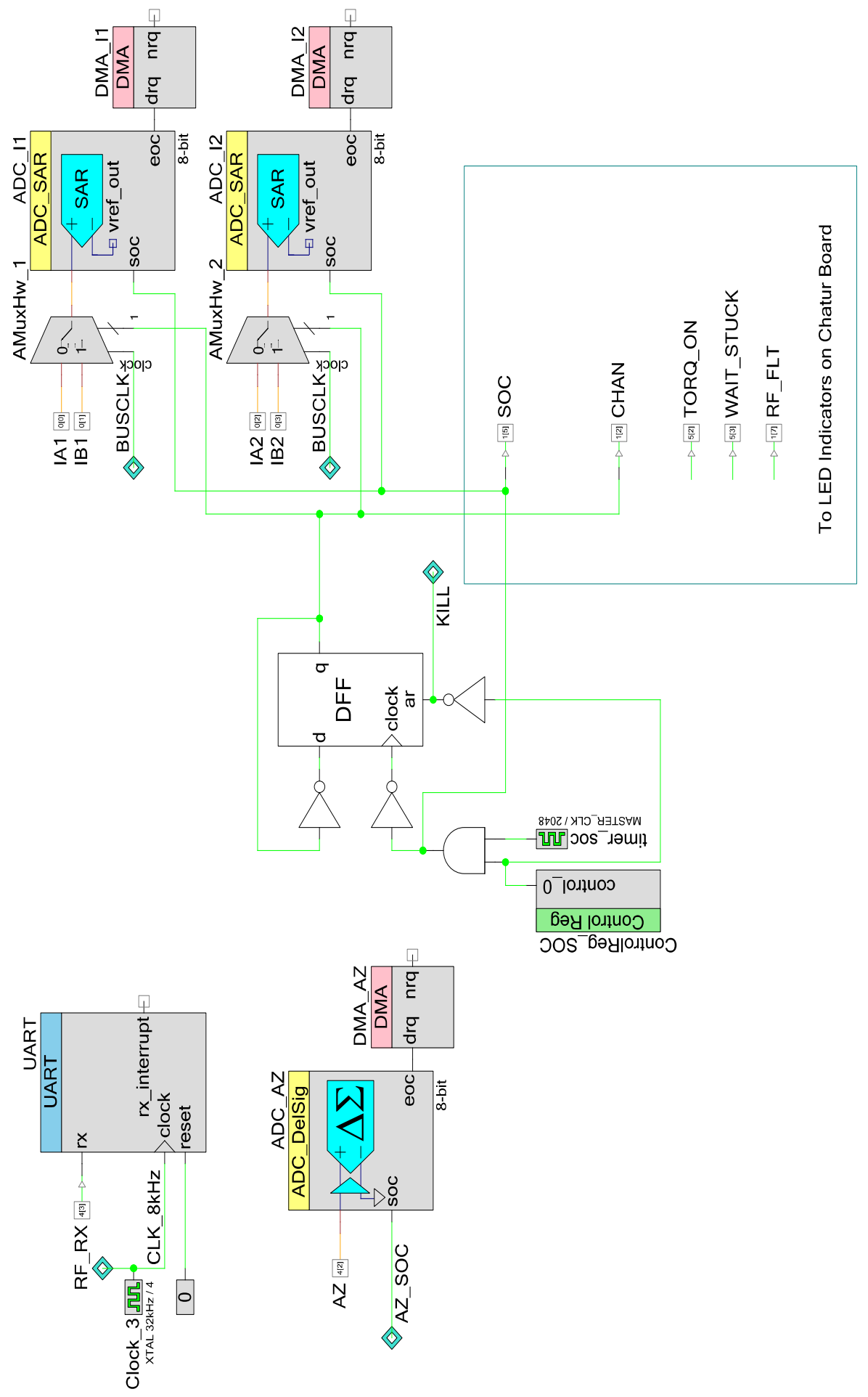

Figure B.17: PSoC ${ }^{\circledR}$ top-level schematics (Page-2): ADCs and UART. 


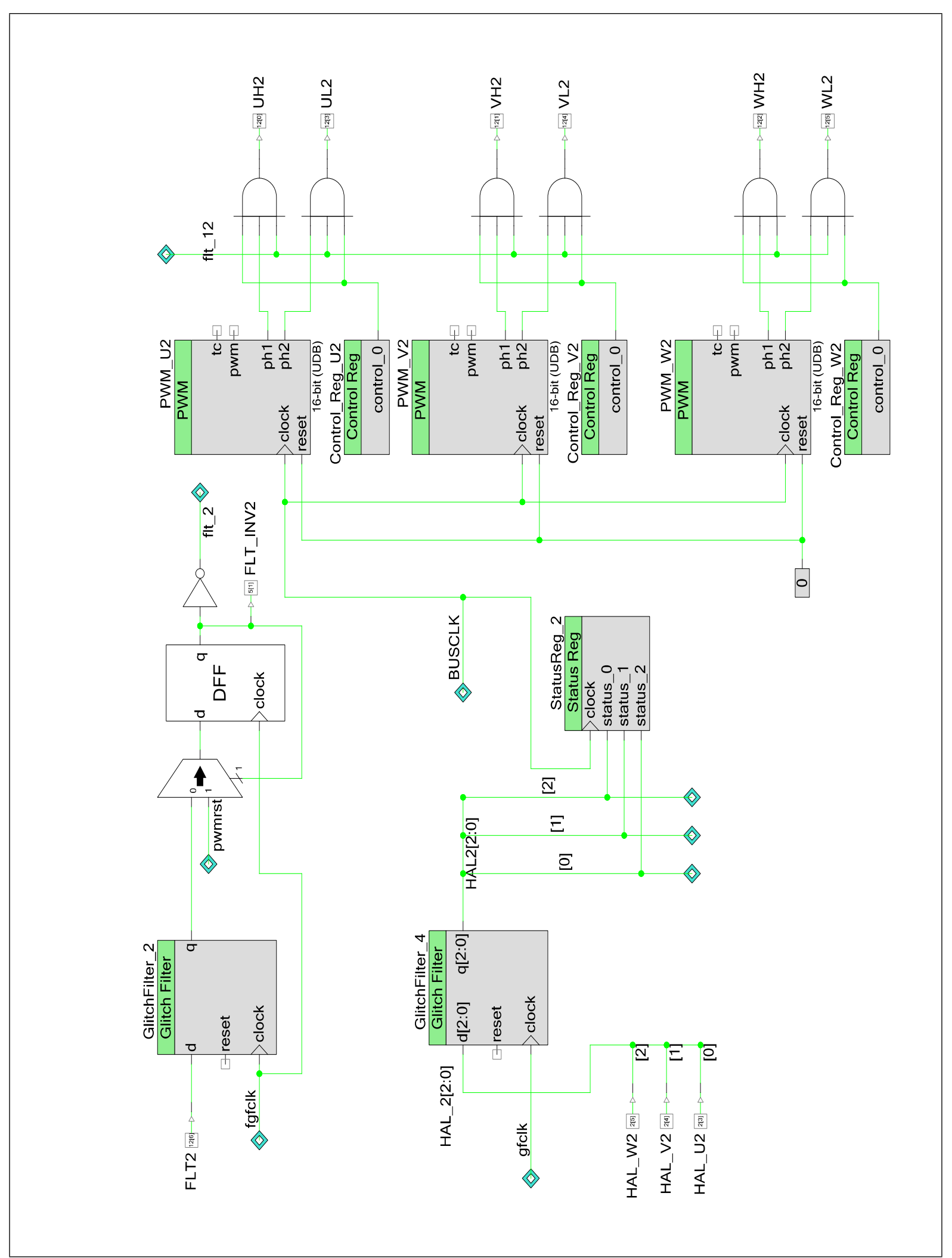

Figure B.18: PSoC $^{\circledR}$ top-level schematics (Page-3): Fault servicing, position sensing and PWM generation for motor-2. 

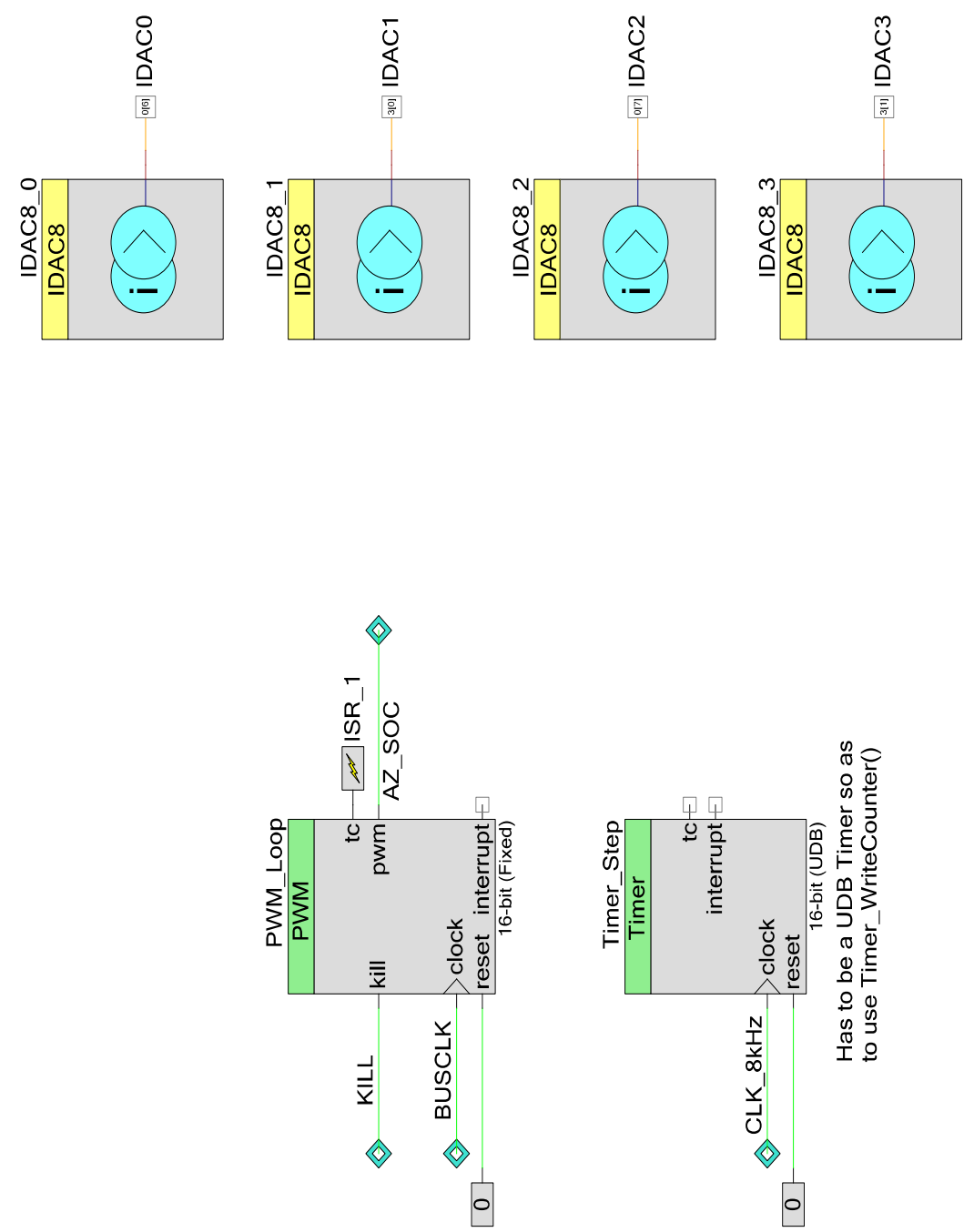

Figure B.19: PSoC $^{\circledR}$ top-level schematics (Page-4): Timers and IDACs. 
* Copyright

* Lalit Patnaik (plalit@dese.iisc.ernet.in, lalit.patnaik@gmail.com)

* PhD Scholar

* Department of Electronic Systems Engineering

* Indian Institute of Science

* Bangalore - 560012

* August 2014

* All Rights Reserved

12

* UNPUBLISHED, LICENSED SOFTWARE.

* CONFIDENTIAL AND PROPRIETARY INFORMATION

* WHICH IS THE PROPERTY OF IISc.

17

$* 1$

// THIS CODE IS FOR USE IN CHATUR BOARD (PSoC based controller board for the legged vehicle chatur)
/* Define and initialize all parameters */

\#define VMIN $1000 / /$ add a dc offset of 1000 to avoid overflow at lower end

\#define Ki1 $0.0045 / / 0.0045$ for loop-time=100us

\#define Kp1 $0.03 \quad / / 0.03$ for loop_time=100us

\#define Ki2 0.0045

\#define Kp2 0.03

32 \#define Ksync 1020 //Gain used for scaling lag between motors to get the wheg sync term \#define ALPHA 0.6

\#define WAIT 0

\#define CALC 1

\#define BLINDFOLD_CNT $30311 / /$ 32768-2457(subtract 300ms)

e A_THRESH 125

\#define K1 $85 / / 96 \Rightarrow \max 12 \mathrm{~A}$ (when $\mathrm{ref}=255) ; 88 \Rightarrow \max 11 \mathrm{~A} ; 85 \Rightarrow \max 10.6 \mathrm{~A}$

\#define K2 85

\#define N1 32768 // Step-timer max value (running at $8192 \mathrm{~Hz}$, gives a time-period of $4 \mathrm{~s}$ )

\#define D_IS 9 // Defines duty factor (spatial; not temporal) for pulsed torque (d_is)

$/ / \mathrm{d}_{-}$is $<4$ (out of 8 ) $\Rightarrow$ Regime -1

$/ / \mathrm{d}_{-}$is $=4$ (out of 8$) \Rightarrow$ Regime -2

$/ / 4<\mathrm{d}_{-}$is $_{1}<8$ (out of 8 ) $\Rightarrow$ Regime -3

$/ / \mathrm{d}_{-}$is $>=8$ (out of 8$) \Rightarrow$ Regime $-4 / 5$

47

$/ / * * * * * * * * * * * * * * * * * * * * * * * * * * * * * * * * * * * * * * * * * * * * * * * * * * * * * * * * * * * * * * * * * * * * * * * * * * * * * * * * * * * * * *$

* Define and initialize global variables

uint 16 vint $1=1000$, vc $1=1000$;

uint 16 vint $2=1000$, vc $2=1000$

uint16 Ifb $1=0$, Ifb 1 old $=0$, Ifb $2=0$, Ifb 2 old $=0$;

uint16 Ia 1 , Ib 1 ;

uint 16 Ia $2, \quad \mathrm{Ib} 2$;

uint16 I1 $[2]=\{0\}$;

uint16 I2 $[2]=\{0\}$

57 uint 8 chan $1=0$, $\operatorname{chan} 2=0 ; / /$ channel selectors for AMuxes

uint 8 flag $=$ WAIT

uint8 icnt1=0, icnt2 $=0$; // large-difference counters for glitch filters

uint 16 az $[2]=\{0\}$;

62

void picon1(uint16 iref, uint16 ifb)

int16 epi; // error can go negative as well!

uint16 vcont;

67 epi $=$ iref-ifb; // error calculation

vint $1=$ vint $1+\mathrm{Ki} 1 *$ epi

// limiter for integrator

if (vint $1>=$ VMAX)

vint $1=$ VMAX

else 
\{
if $\quad($ vint $1<=$ VMIN $)$
vint $1=$ VMIN;

77

\}

$\mathrm{vcont}=\operatorname{vint} 1+\mathrm{Kp} 1 * \mathrm{ep} ;$

// limiter for pi controller

if $(v \operatorname{vcont}>=\mathrm{VMAX})$

$\mathrm{vc} 1=\operatorname{VMAX}$

else

82

\{

if $(\operatorname{vcont}<=$ VMIN $)$

$\mathrm{vc} 1=\mathrm{VMIN}$

else

$\mathrm{vc} 1=\mathrm{vcont}$

87

\}

\}

void picon2(uint16 iref, uint16 ifb)

\{

92 int16 epi; // error can go negative as well!

uint16 vcont;

epi $=$ iref-ifb; // error calculation

vint $2=$ vint $2+\mathrm{Ki} 2 *$ epi

// limiter for integrator

97 if (vint $2>=$ VMAX)

vint 2 = VMAX;

else

\{

if $($ vint $2<=$ VMIN $)$

102

vint $2=$ VMIN

\}

vcont $=$ vint $2+\mathrm{Kp} 2 * \mathrm{epi}$

// limiter for pi controller

if $($ vcont $>=$ VMAX)

107

$\mathrm{vc} 2=\operatorname{VMAX}$

else

\{

if $(v \operatorname{vcont}<=\mathrm{VMIN})$

$\mathrm{vc} 2=\mathrm{VMIN}$

112 else

$\mathrm{vc} 2=\mathrm{vcont}$;

\}

117

void glitch_filter1()

\{

if (Ifb1>Ifb1old)

122

if $(($ Ifb1-Ifb1old $)>8000) / /(8000 / 2 \wedge 12) * 2 \mathrm{~A} \sim 4 \mathrm{~A}$ increase in one $\mathrm{s} / \mathrm{w}$ loop time \{

icnt $1=$ icnt $1+1$;

if $\quad($ icnt $1<3)$

If $1=$ Ifb 1 old ;

127

$$
\text { else }\{\quad \text { icnt } 1=0 ;
$$

132

else

if $(($ Ifb1old-Ifb1) >8000) // $\sim 4$ decrease in one $\mathrm{s} / \mathrm{w}$ loop time

\{

137

$\mathrm{icnt} 1=\mathrm{icnt} 1+1$;

if $\quad($ icnt $1<3)$

$\mathrm{Ifb} 1=\mathrm{Ifb} 1 \mathrm{old} ;$

142

icnt1 $=0$ 
147

152

157

167

172

177

182

187

192

197

202

207

212

void glitch_filter2()

\{

if (Ifb $2>$ Ifb 2 old )

if $(($ Ifb $2-$ Ifb $2 \circ \mathrm{ld})>8000)$

\{

icnt $2=$ icnt $2+1$

if $($ icnt $2<3)$

Ifb $2=$ Ifb 2 old

\}

else

icnt2 $=0$

\}

icnt2 $=0$

els e

$(($ Ifb 2 old $-I f b 2)>8000)$

icnt $2=$ icnt $2+1$;

if (icnt $2<3$ )

Ifb $2=$ Ifb $2 \circ \mathrm{ld}$

\}

else

icnt $2=0$

void main ()

$/ / * * * * * * * * * * * * * * * * * * * * * * * * * * * * * * * * * * * * * * * * * *$
$/ *$ Define and initialize all variables $* /$

uint8 secno1, secno1old, secno1older, secno2, secno2old, secno2older;

$/ /$ secnoxold = sector no. in previous s/w cycle

$/ /$ secnoxolder $=$ previous sector from which present sector was arrived at

uint 8 ref, RxDataOld=0, err_cnt=0, Iref18, Ifb18, Iref28, Ifb28;// vc28, Ifb18, Ifb28, vc18, vint 18 ;

int 16 Iref 1, Iref $2, \quad$ Iref 1 avg $=0, \quad$ Iref 2 avg $=0 ; / /$ olvc ;

int 16 Iref 1 sync $=0$, Iref 2 sync $=0$;

int 8 ews1, ews $2 ; / /$ error in wheg sync

uint 16 Ifb 1 avg $=0, \quad$ If 2 avg $=0$;

$/ /$ uint $16 \operatorname{mid}=29492 ; / / 32768-3276$ (subtract $400 \mathrm{~ms}$ )

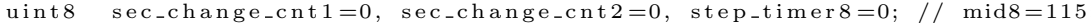

uint16 impact_cnt $=0$;

uint16 step_timer $=0$;

$/ / * * * * * * * * * * * * * * * * * * *$

/* Enable UART component */

UART_Start ( ) ;

/* Enable global interrupts */

CyGlobalintEnable:

/* Enable IDACs */

ID AC8_0_Start ( ) ; // IDAC0

ID AC8_1_Start ( ) ; // IDAC1

IDAC8_2_Start (); // IDAC2

IDAC8_3_Start ( ) ; // IDAC3

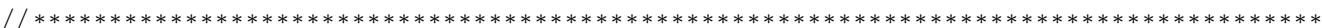

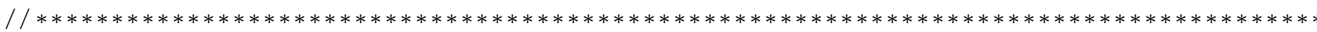

/* Configure and enable DMAs*/

/* Variable declarations for DMA_I1 */

uint 8 DMA_I1_Chan

uint8 DMA_I1_TD [1] ; 
/ DMA Configuration for DMA_I1 */

\#define DMA_I1_BYTES_PER_BURST 2

\#d efine DMA_I1_REQUEST_PER_BURST 1

\#define DMA_I1_SRC_BASE (CYDEV_PERIPH_BASE)

\#define DMA_I1_DST_BASE (CYDEV_SRAM_BASE)

DMA_I1_Chan = DMA_I1_DmaInitialize (DMA_I1_BYTES_PER_BURST, DMA_I1_REQUEST_PER_BURST, HI16 (DMA_I1_SRC_BASE) , HI16 (DMA_I1_DST_BASE) ) ;

DMA_I1_TD $[0]=$ CyDmaTdAllocate () ;

CyDmaTdSetConfiguration (DMA_I1_TD [0] ， 4， DMA_I1_TD [0] ， TD_INC_DST_ADR) ;

CyDmaTdSetAddress (DMA_I1_TD [0], LO16 ( ( u int 32 ) ADC_I1_SAR_WRK0_PTR), LO16 ( ( uint 32 ) I1 )) CyDmaChSetInitialTd (DMA_I1_Chan, DMA_I1_TD [0]) ;

CyDmaChEnable (DMA_I1_Chan, 1) ;

/* Variable declarations for DMA_I2 */

/* Move these variable declarations to the top of the function */

uint 8 DMA_I2_Chan;

uint 8 DMA_I2_TD [1]

/* DMA Configuration for DMA_I2 */

\#define DMA_I2_BYTES_PER_BURST 2

\#define DMA_I2_REQUEST_PER_BURST 1

\#d efine DMA_I2_SRC_BASE (CYDEV_PERIPH_BASE)

\#define DMA_I2_DST_BASE (CYDEV_SRAM_BASE)

DMA_I2_Chan = DMA_I2_DmaInitialize (DMA_I2_BYTES_PER_BURST, DMA_I2_REQUEST_PER_BURST,

HI16 (DMA_I2_SRC_BASE) , HI16 (DMA_I2_DST_BASE) ) ;

DMA_I2_TD $[0]=$ CyDmaTdAllocate () ;

CymaTdSetConfiguration (DMA_I2_TD [0], 4, DMA_I2_TD [0], TD_INC_DST_ADR);

CyDmaTdSetAddress (DMA_I2_TD [0] , LO16 ( ( u int 32 ) ADC_I2_SAR_WRK0_PTR), LO16 ( ( u int 32 ) I 2 )) ; CyDmaChSetInitialTd (DMA_I2_Chan, DMA_I2_TD [0]);

CyDmaChEnable(DMA_I2_Chan, 1);

/* Variable declarations for DMA_AZ */

/* Move these variable declarations to the top of the function */

uint 8 DMA_AZ_Chan;

uint8 DMA_AZ_TD [1]

/* DMA Configuration for DMA_AZ */

\#d e fine DMA_AZ_BYTES_PER_BURST 1

\#define DMA_AZ_REQUEST_PER_BURST 1

\#d efine DMA_AZ_SRC_BASE (CYDEV_PERIPH_BASE)

\#define DMA_AZ_DST_BASE (CYDEV_SRAM_BASE)

DMA_AZ_Chan = DMA_AZ_DmaInitialize (DMA_AZ_BYTES_PER_BURST, DMA_AZ_REQUEST_PER_BURST,

HI16 (DMA_AZ_SRC_BASE) , HI16 (DMA_AZ_DST_BASE))

DMA_AZ_TD $[0]=$ CyDmaTdAllocate () ;

CyDmaTdSetConfiguration (DMA_AZ_TD [0] , 2, DMA_AZ_TD [0] , 0);

CyDmaTdSetAddress (DMA_AZ_TD [0]， LO16 ( ( u int 32 ) ADC_AZ_DEC_SAMP_PTR)，LO16 ( (uint 32 ) az )) ;

CyDmaChSetInitialTd (DMA_AZ_Chan, DMA_AZ_TD [0]) ;

CyDmaChEnable (DMA_AZ_Chan, 1);

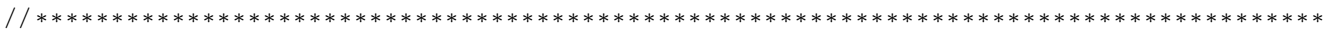

$/ / * * * * * * * * * * * * * * * * * * * * * * * * * * * * * * * * * * * * * * * * * * * * * * * * * * * * * * * * * * * * * * * * * * * * * * * * * * * * * * * * * * * * *$

/* Enable ADCs */

ADC_I1_Start ( ) ;

ADC_I1_IRQ_Disable ();

ADC_I $2_{-}$Start () ;

ADC_I2_IRQ_Disable ( ) ;

ADC_AZ_Start () ;

ADC_AZ_IRQ_Disable ();

ControlReg_SOC_Write (1);

/* Enable PWM blocks but keep their outputs disabled */

PWM_U1_Start ();

PWM_V1_Start ();

PWM_W1_Start ();

PWM_U2_Start ();

PWM_V2_Start ();

PWM_W2_Start ( ) ;

/* Enable ISR for transition from WAIT state to CALC state */

ISR_1_Start ( ) ;

CyDelayUs (750); // wait till 'things', settle down

PWM_Loop_Start (); // Start software loop timer (100us) 
/* Get position (sector no.) from hall sensors */

secnolold = StatusReg_1_Read (); // this variable stores the sector no. during the last s/w cycle

else

\{

err $\mathrm{r}_{-} \mathrm{cnt}=0$;

RF_FLT_DR $=($ RF_FLT_DR \& $\sim$ RF_FLT_MASK $) \quad((0<<$ RF_FLT_SHIFT $) \quad \&$ RF_FLT_MASK $)$; 


\section{$/ / * * * * * * * * * * * * * * * * * * * * * * * * * * * * * * * * * * * * * * * * * * * * * * * * * * * * * * * * * * * * * * *)$
$/ /$ ref $=1400 ; / /$ for use temporarily; remove when rf link is up \\ $/ /$ olvc $=$ ref $<<3 ; / /$ open loop vc; remove during closed loop operation!}

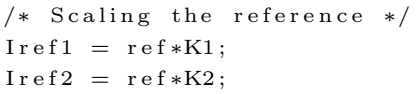


if $($ err_cnt $>1) / / 1$

\{ $\mathrm{ref}=0$;

// Glow LED at Pin RF_FLT

RF_FLT_DR $=\left(\right.$ RF_FLT_DR \& ${ }^{\sim}$ RF_FLT_MASK $) \mid((1<<$ RF_FLT_SHIFT $)$ \& RF_FLT_MASK $)$; \} else

$/ /$ ref $=1400 ; / /$ for use temporarily; remove when RF link is up!

$/ /$ olvc $=$ ref $<<3 ; / /$ open loop vc; remove during closed loop operation!

/* Scaling the received ref (uint8) value to get Irefs (uint16; 4.12 format; Ibase=2A) */

Iref $1=$ ref $* \mathrm{~K} 1 ; / / 96 \Rightarrow \max 12 \mathrm{~A}$

Iref $2=$ ref $*$ K2

step_timer $=$ Timer_Step_ReadCounter () ;

\} // end of between-impacts code

/* Wheg synchronization */

Iref $1+=$ Ireflsync;

Iref $2+=$ Iref $2 \mathrm{sync}$

/* Limit Iref to $11 \mathrm{~A}(255 * 88) * /$

if (Iref $1>=22000)$

Iref $1=22000$

if $($ Iref $1<=0)$

Iref $1=0$;

if $($ Iref $2>=22000)$

Iref $2=22000$

if $($ Iref $2<=0)$

Iref $2=0$;

if $(r$ ef $==0)$

\{

Ir ef $1=0$;

Ir ef $2=0$;

Iref $1 \mathrm{sync}=0$;

Iref 2 sync $=0$;

\}

/* Get position (sector no.) from hall sensors */

secno1 = StatusReg-1_Read ();

secno $2=$ StatusReg_2_Read ()

if (secnol != secnolold)

\{

secnololder = secnolold; // remember previous sector

\}

if (secno2 != secno2old)

secno2older $=$ secno2old; // remember previous sector

// ! ! ! ! ! ! ! ! ! ! ! ! ! ! ! ! ! ! ! ! ! ! ! ! ! ! ! ! ! ! ! ! ! ! ! ! ! ! ! ! ! ! ! ! ! ! ! ! ! ! ! ! ! ! ! ! ! ! ! ! ! ! !

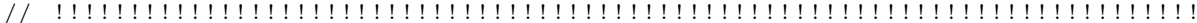

$/ *$ Sector change count */

if $(((\operatorname{secnolold}==5) \& \&(\operatorname{secno} 1==4)) \quad||((\operatorname{secno1old}==4) \& \&(\operatorname{secno} 1==6))|| \backslash$

$((\operatorname{secno1old}==6) \& \&(\operatorname{secno} 1==2))|| \quad((\operatorname{secno} 10 l d==2) \& \& \quad(\operatorname{secno} 1==3)) \quad||$

$((\operatorname{secnolold}==3) \& \&(\operatorname{secno} 1==1))||((\operatorname{secnolold}==1) \& \& \quad(\operatorname{secno} 1==5)))$

\{

$\mathrm{sec}_{-} \mathrm{change} \mathrm{e}_{-} \mathrm{cnt} 1++$

\}

if $(((\operatorname{secno} 2 \circ 1 d==5) \& \&(\operatorname{secno} 2==1)) \quad||((\operatorname{secno} 2 o l d==1) \& \& \quad(\operatorname{secno} 2==3)) \quad||$ 
$((\operatorname{secno} 2 o l d==3) \& \&(\operatorname{secno} 2==2)) \quad||((\operatorname{secno} 2 o l d==2) \& \&(\sec n o 2==6))||$

$((\operatorname{secno} 2 \circ 1 d==6) \& \&(\operatorname{secno} 2==4)) \quad|| \quad((\sec n o 2 \circ l d==4) \& \& \quad(\sec n o 2==5)))$

$\mathrm{sec}_{-} \mathrm{change} \mathrm{e}_{-} \mathrm{cnt} 2++$; \}

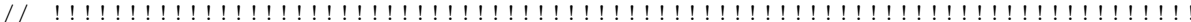
$/ /$ ! ! ! ! ! ! ! ! ! ! ! ! ! ! ! ! ! ! ! ! ! ! ! ! ! ! ! ! ! ! ! ! ! ! ! ! ! ! ! ! ! ! ! ! ! ! ! ! ! ! ! ! ! ! ! ! ! ! ! ! !

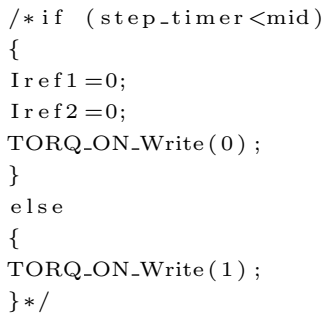

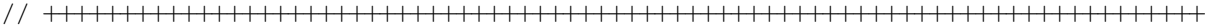

$/ /+1111111111111111111111111111111111111111111111111111111111111111111111111+$

/* 1. Rectify, level-shift, scale and filter measured currents

2. Generate proper duty ratios using PI controller

3. Activate PWM in appropriate phases (based on sector no.) */

$/ /$ For motor-1 (OCW) i.e. right motor while moving forward switch $(\sec n o 1)$

\{

case $0 \times 5$ :

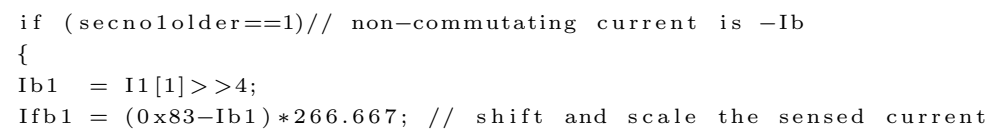


577

if ( secnololder $==5$ ) // non-commutating current is Ia

\{

Ia $1=\mathrm{I} 1[0]>>4$;

Ifb1 $=($ Ia $1-0 \times 82) * 266.667 ; / /$ shift and scale the sensed current

\}

else// non-commutating current is - Ic

\{

$\mathrm{Ia} 1=\mathrm{I} 1[0]>>4$;

$\mathrm{Ib} 1=\mathrm{I} 1[1]>>4$;

Ifb1 $=(\operatorname{Ia} 1+\operatorname{Ib} 1-0 \times 105) * 266.667 ; / /$ shift and scale the sensed current \}

glitch_filter 1()$; / /$ get rid of false glitches

Ifb 1 avg $+=($ Ifb $1-$ Ifblavg $) *$ ALPHA; // EMA filter

picon 1 ( Iref 1 avg, Ifb 1 avg) ; // Ifb 1 avg

Control_Reg_V1_Write (0);

//PWM_V1_Stop ( ) ;

PWM_W1_WriteCompare (0);

Control_Reg_W1_Write (1); / /PWM_W1_Enable ();

PWM_U1_WriteCompare (vc1-1000); // ref or vc1-1000

Control_Reg_U1_Write (1);

break: //PWM_U1_Enable ( ) :

case $0 \times 6$ :

\{

$\mathrm{Ia} 1=\mathrm{I} 1[0]>>4$;

$\mathrm{Ib} 1=\mathrm{I} 1[1]>>4 ;$

Ifb $1=(\operatorname{Ia} 1+\mathrm{Ib} 1-0 \times 105) * 266.667 ; / /$ shift and scale the sensed current \}

else// non-commutating current is Ib

$\mathrm{Ib} 1=\mathrm{I} 1[1]>>4$;

Ifb1 $=(\operatorname{Ib} 1-0 \times 83) * 266.667 ; / /$ shift and scale the sensed current

\}

glitch_filter 1()$; / /$ get rid of false glitches

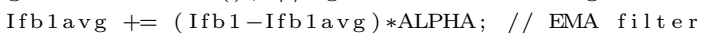

picon1 (Iref1avg, Ifb1avg); // Ifb1avg

Control_Reg_U1_Write (0);

//PWM_U1_Stop ( ) ;

PWM_W1_WriteCompare (0);

Control_Reg_W1_Write (1); //PWM_W1_Enable ( ) ;

PWM_V1_WriteCompare (vc1-1000); // r ef or vc1-1000

Control_Reg_V1_Write (1);

break;

//PWM_V1_Enable ();

case $0 \times 2$ :

if $($ secnololder $==6) / /$ non-commutating current is Ib

\{

$\mathrm{Ib} 1=\mathrm{I} 1[1]>>4$;

Ifb1 $=(\operatorname{Ib} 1-0 \times 83) * 266.667 ; / /$ shift and scale the sensed current

\}

else // non-commutating current is -Ia

\{

$\mathrm{Ia} 1=\mathrm{I} 1[0]>>4$;

$\mathrm{Ifb} 1=(0 \times 82-\mathrm{Ia} 1) * 266.667$;

\}

glitch_filter 1()$; / /$ get rid of false glitches

Ifblavg $+=($ Ifb1-Ifb1avg) *ALPHA; // EMA filter

picon 1 ( Ireflavg, Ifb1avg); // Ifb 1 avg

Control_Reg_W1_Write (0);

//PWM_W1_Stop ( ) ;

PWM_U1_WriteCompare (0);

Control_Reg_U1_Write (1);

/ /PWM_U1_Enable ( ) ;

PWM_V1_WriteCompare (vc1-1000); // ref or vc1-1000

Control_Reg_V1_Write (1); break ; //PWM_V1_Enable ();

case $0 \times 3$ :

if $($ secnololder $==2$ )// non-commutating current is $-I$ a 
$\mathrm{Ia} 1=\mathrm{I} 1[0]>>4$;

Compare ( vc1

break ; / / PWM_W1_Enable ( ) ;

case $0 \times 1$ :

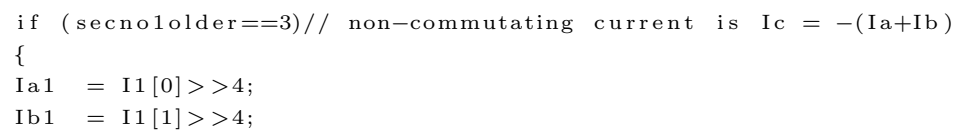

// For motor-2 (CW) i.e. left motor while moving forward switch (secno2) 
picon 2 ( Iref 2 avg, Ifb 2 avg) ; / / Ifb 2 avg

Control_Reg_W2_Write (0);

//PWM_W2_Stop (); // disable W leg

PWM_U2_WriteCompare (0); // latch V leg to $\mathrm{N}$

Control_Reg_U2_Write ( 1 ); / /PWM_U2_Enable ( )

PWM_V2_WriteCompare(vc2-1000); // switch U leg // ref or vc2-1000 Control_Reg_V2_Write (1);

break ;

/ /PWM_V2_Enable ( ) ;

case $0 \times 4$ :

if (

\{

$\mathrm{Ia} 2=\mathrm{I} 2[0]>>4$;

Ib $2=\mathrm{I} 2[1]>>4 ;$

Ifb2 $2=(0 \times 106-\mathrm{Ia} 2-\mathrm{Ib} 2) * 266.667 ; / /$ shift and scale the sensed current

else // non-commutating current is -Ia

I $2=\mathrm{I} 2[0]>>4 ;$

Ifb2 $=(0 \times 83-\mathrm{Ia} 2) * 266.667 ; / /$ shift and scale the sensed current

\}

glitch_filter 2()$; / /$ get rid of false glitches

Ifb 2 avg $+=($ Ifb $2-$ Ifb 2 avg $) *$ ALPHA; // EMA filter

picon 2 ( Iref 2 avg, Ifb 2 avg ); // Ifb 2 avg

Control_Reg_V2_Write $(0)$;

/ /PWM_V2_Stop ( ) ;

PWM_U2_WriteCompare (0);

Control_Reg_U2_Write (1);

/ / PWM_U2_Enable ( ) ;

PWM_W2_WriteCompare (vc2-1000); // ref or vc2-1000

Control_Reg_W2_Write (1);

break;

/ / PWM_W2_Enable ( ) ;

case $0 \times 6$ :

if $($ secno2older $==2) / /$ non-commutating current is $-I b$

\{

$\mathrm{Ib} 2=\mathrm{I} 2[1]>>4$;

Ifb $2=(0 \times 83-$ Ib2 $) * 266.667 ; / /$ shift and scale the sensed current

// Note: Ib has a 1 -bit zero error; hence $0 \times 7 F$ instead of $0 \times 80$

\}

else // non-commutating current is $\mathrm{Ic}=-(\mathrm{I} \mathrm{a}+\mathrm{Ib})$

\{

I $2=$ I $2[0]>>4 ;$

$\mathrm{Ib} 2=\mathrm{I} 2[1]>>4 ;$

Ifb $2=(0 \times 106-\mathrm{Ia} 2-\mathrm{Ib} 2) * 266.667 ; / /$ shift and scale the sensed current

\}

glitch_filter 2()$; / /$ get rid of false glitches

Ifb 2 avg $+=($ Ifb $2-$ Ifb 2 avg $) *$ ALPHA; // EMA filter

picon 2 ( Iref 2 avg, Ifb 2 avg ) ; // Ifb 2 avg

Control_Reg_U2_Write (0);

//PWM_U2_Stop ( ) ;

PWM_V2_WriteCompare (0);

Control_Reg_V2_Write (1); / / PWM_V2_Enable ( ) ;

PWM_W2_WriteCompare (vc2-1000); // ref or vc2-1000

Control_Reg_W2_Write (1);

break ;

/ / PWM_W2_Enable ();

case $0 \times 2$ :

if (secno2older $==3$ ) // non-commutating current is Ia

\{

$\mathrm{Ia} 2=\mathrm{I} 2[0]>>4$;

Ifb $2=($ Ia $2-0 \times 83) * 266.667 ; / /$ shift and scale the sensed current

\}

else // non-commutating current is -Ib

$\mathrm{Ib} 2=\mathrm{I} 2[1]>>4$;

Ifb $2=(0 \times 83-\mathrm{Ib} 2) * 266.667 ; / /$ shift and scale the sensed current \}

glitch_filter 2()$; / /$ get rid of false glitches

Ifb 2 avg $+=($ Ifb $2-$ Ifb 2 avg $) *$ ALPHA; // EMA filter 
picon $2($ Ireff 2 avg, Ifb 2 avg ); / / If 2 avg

Control_Reg_W2_Write (0);

//PWM_W2_Stop ( ) ;

PWM_V2_WriteCompare (0);

Control_Reg_V2_Write (1);

//PWM_V2_Enable ()

PWM_U2_WriteCompare (vc2-1000); // ref or vc2-1000

Control_Reg_U2_Write ( 1 );

break ;

/ PWM_U2_Enable ( )

case $0 \times 3$ :

if ( secno2older $==1$ )// non-commutating current is $-\mathrm{Ic}=\mathrm{Ia}+\mathrm{Ib}$

\{

$\mathrm{Ia} 2=\mathrm{I} 2[0]>>4$

$\mathrm{Ib} 2=\mathrm{I} 2[1]>>4$;

Ifb2 $2=(\operatorname{Ia} 2+\operatorname{Ib} 2-0 \times 106) * 266.667 ; / /$ shift and scale the sensed current

else // non-commutating current is Ia

$\mathrm{Ia} 2=\mathrm{I} 2[0]>>4$

$\mathrm{Ia} 2=\mathrm{I} 2[0]>>4 ;$
$\mathrm{Ifb} 2=(\mathrm{Ia} 2-0 \times 83) * 266.667 ; / /$ shift and scale the sensed current

glitch_filter 2()$; / /$ get rid of false glitches

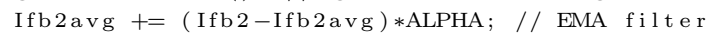

picon 2 ( Iref 2 avg, Ifb 2 avg ); // Ifb 2 avg

Control_Reg_V2_Write $(0)$

//PWM_V2_Stop ( ) ;

PWM_W2_WriteCompare ( 0$)$;

Control_Reg_W2_Write (1);

/ / PWM_W2_Enable ()

PWM_U2_WriteCompare(vc2-1000); // ref or vc2-1000

Control_Reg_U2_Write (1);

break ;

/ / PWM_U2_Enable ( ) ;

case $0 \times 1$ :

if (secno2older==5)// non-commutating current is Ib

\{

$\mathrm{Ib} 2=\mathrm{I} 2[1]>>4$

Ifb $2=(\operatorname{Ib} 2-0 \times 83) * 266.667 ; / /$ shift and scale the sensed current

\}

else // non-commutating current is - Ic

\{

$\mathrm{Ia} 2=\mathrm{I} 2[0]>>4$

$\mathrm{Ib} 2=\mathrm{I} 2[1]>>4$

Ifb2 $2=(\operatorname{Ia} 2+\operatorname{Ib} 2-0 \times 106) * 266.667 ; / /$ shift and scale the sensed current

glitch_filter2(); // get rid of false glitches

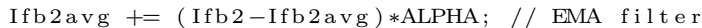

picon $2($ Iref 2 avg, Ifb 2 avg ) ; / Ifb 2 avg

Control_Reg_U2_Write $(0)$

//PWM_U2_Stop ( ) ;

PWM_W2_WriteCompare (0);

Control_Reg_W2_Write (1);

/ PWM_W2_Enable ()

PWM_V2_WriteCompare (vc2-1000); // ref or vc2-1000

Control_Reg_V2_Write (1);

break ;

/ / PWM_V2_Enable ( ) ;

case $0 \times 0$

break;

default :

PWM_U2_Stop ( ) ;

PWM_V2_Stop ( ) ;

PWM_W2_Stop ( )

$1 / \mathrm{n} 111111111111111111111111111111111111111111111111111111111111111111111111111+$ // H111111111111111111111111111111111111111111111111111111111111111111+

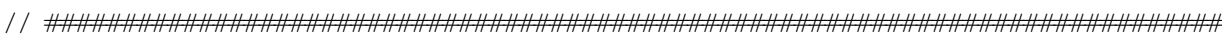
/* Send debug waveforms to IDACs (For Diagnostics) */ 
862

867

872

877

882

887

892

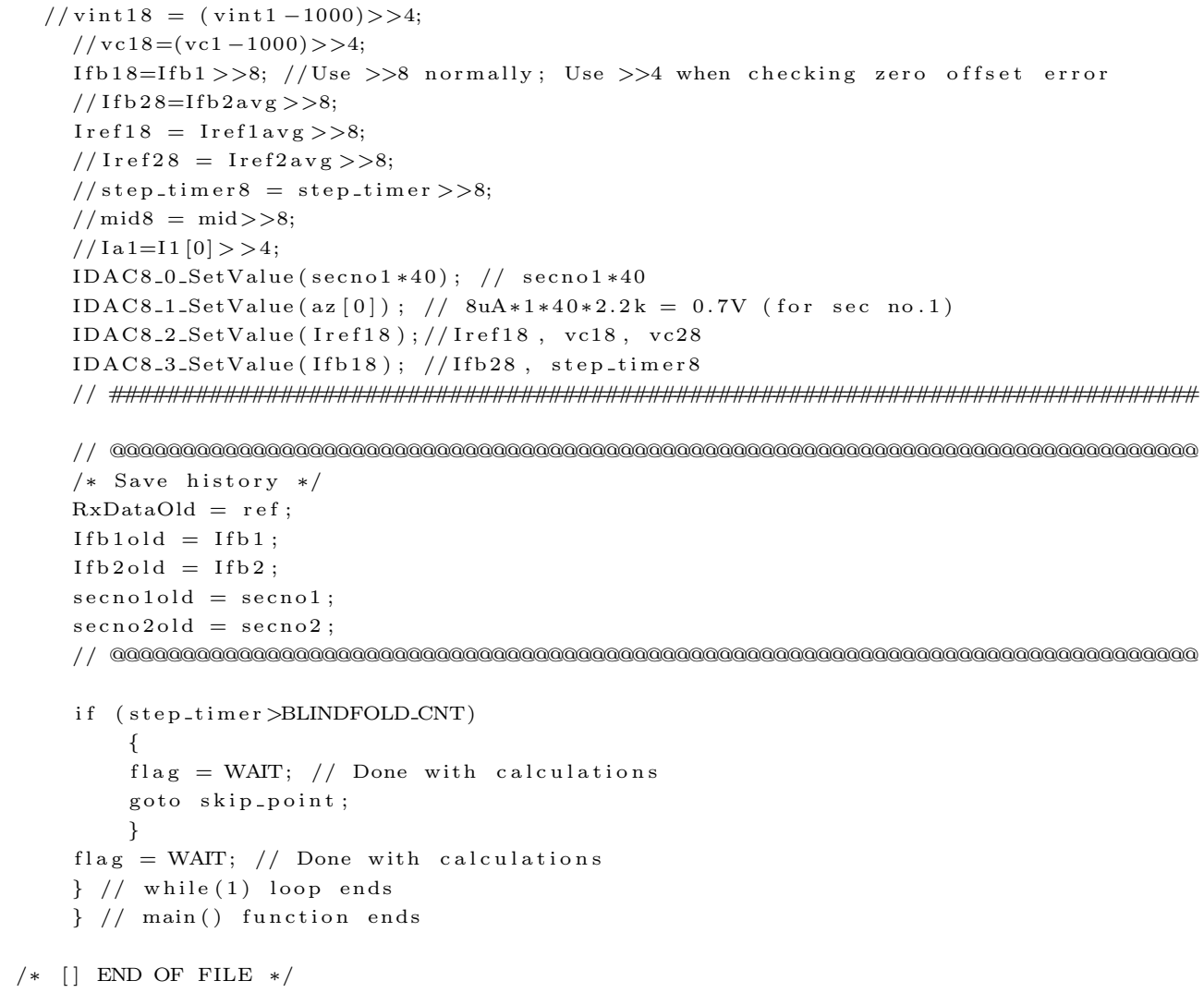

Listing B.2: C-code for the control algorithm that is executed by PSoC ${ }^{\circledR}$ 's ARM core. 


\section{Appendix C}

\section{List of Publications}

1. Lalit Patnaik and Loganathan Umanand, "Torque regimes in a rimless wheel based 2D dynamic walker using pulsed torque actuation," under review, IEEE Transactions on Robotics.

2. Lalit Patnaik and Loganathan Umanand, "A control topology for pulsed torque actuation of a synchronized dual BLDC motor driven platform with cyclically varying load torque," under review, IET Power Electronics.

3. Lalit Patnaik and Loganathan Umanand, "Modeling of constraints, fundamental limits and optimal locus of operating points in velocity-angle space for an inverted pendulum based actuated dynamic walker," manuscript submitted, Simulation Modelling Practice and Theory.

4. Lalit Patnaik and Loganathan Umanand, "A dynamic model for the Jansen leg mechanism using bondgraphs," manuscript under preparation. 


\section{Bibliography}

Alexander, R. M. Mechanics of bipedal locomotion. In Davies, P. Spencer and Sunderland, N., editors, Perspectives in Experimental Biology, page 493. Pergamon Press, 1976.

Alexander, R. M. Optimization and gaits in the locomotion of vertebrates. Physiological reviews, 69(4):1199-1227, 1989.

Alexander, R. M. Simple models of human movement. ASME Applied Mechanics Reviews, 48(8):461-470, August 1995.

Alexander, R. M. Walking and running. The Mathematical Gazette, 80(488):pp. 262-266, 1996.

Alexander, R. M. Principles of Animal Locomotion. Princeton University Press, 2003.

Alexander, R. M. and Jayes, A. S. Fourier analysis of forces exerted in walking and running. Journal of Biomechanics, 13(4):383-390, 1980.

Allen, Thomas J.; D.Quinn, Roger; Bachmann, Richard J., and Ritzmann, Roy E. Abstracted biological principles applied with reduced actuation improve mobility of legged vehicles. In IEEE/RSJ International Conference on Intelligent Robots and Systems, pages 1370-1375. CWRU, October 2003.

Arikawa, Keisuke and Hirose, Shigeo. Development of quadruped walking robot TITANVIII. In Intelligent Robots and Systems' 96, IROS 96, Proceedings of the 1996 IEEE/RSJ International Conference on, volume 1, pages 208-214. IEEE, 1996.

Asano, F. Dynamic gait generation of telescopic-legged rimless wheel based on asymmetric impact posture. In IEEE-RAS International Conference on Humanoid Robots, December 2009.

Asano, F.; Kawamoto, J., and Paul, Saint. Passive dynamic walking of viscoelastic-legged rimless wheel. May 2012. 
Bechstein, B. U. Improvements in and relating to toys., 1912. UK Patent 7453.

Bekker, M.G. Is the wheel the last word in land locomotion. New Scientist, 248:406-410, 1961.

Bhounsule, Pranav A; Cortell, Jason; Grewal, Anoop; Hendriksen, Bram; Karssen, JG Daniël; Paul, Chandana, and Ruina, Andy. Low-bandwidth reflex-based control for lower power walking: $65 \mathrm{~km}$ on a single battery charge. The International Journal of Robotics Research, 33(10):1305-1321, 2014.

Cavagna, G. A.; Heglund, N. C., and Taylor, C. R. Mechanical work in terrestrial locomotion: two basic mechanisms for minimizing energy expenditure. American Journal of Physiology - Regulatory, Integrative and Comparative Physiology, 233:243-261, 1977.

Cavagna, G A; Willems, P A, and Heglund, N C. The role of gravity in human walking: pendular energy exchange, external work and optimal speed. The Journal of Physiology, 528(3):657-668, 2000.

Cavagna, G. A.; Willems, P. A.; Legramandi, M. A., and Heglund, N. C. Pendular energy transduction within the step in human walking. Journal of Experimental Biology, 205 (21):3413-3422, 2002.

Chen, H.C. and Liaw, C.M. Sensorless control via intelligent commutation tuning for brushless dc motor. IEE Proceedings - Electric Power Applications, 146:678-684(6), November 1999.

Chi, Wen-Chun; Cheng, Ming-Yang, and Chen, Cheng-Hu. Position-sensorless method for electric braking commutation of brushless dc machines. IET Electric Power Applications, 7:701-713(12), November 2013.

Clark, Samuel Kelly and Dodge, Richard N. A handbook for the rolling resistance of pneumatic tires. 1979.

Coleman, Michael J.; Chatterjee, Anindya, and Ruina, Andy. Motions of a rimless spoked wheel: a simple 3D system with impacts. Dynamics and Stability of Systems, 1997.

Collins, Steven Hartley and Ruina, Andy. A bipedal walking robot with efficient and human-like gait. In IEEE International Conference on Robotics and Automation, April 2005. 
Collins, Steven Hartley; Wisse, Martijn, and Ruina, Andy. A three-dimensional passivedynamic walking robot with two legs and knees. The International Journal of Robotics Research, 20(7):607-615, July 2001.

Collins, Steven Hartley; Ruina, Andy; Tedrake, Russ, and Wisse, Martijn. Efficient bipedal robots based on passive-dynamic walkers. Science, 307:1082-1085, February 2005.

Coutermarsh, Barry. Velocity effect of vehicle rolling resistance in sand. Journal of Terramechanics, 44(4):275-291, 2007.

Dertien, Edwin. Realisation of an energy-efficient walking robot. Master's thesis, University of Twente, June 2005.

Dertien, Edwin. Dynamic walking with dribbel. Robotics Automation Magazine, IEEE, 13(3):118-122, sept. 2006.

Diwakar, J. E. Study of dynamics of induction motor driven, gear-coupled linkagemechanism using bond graphs. $\mathrm{PhD}$ thesis, Indian Institute of Science, November 1996.

Fallis, G. T. Walking toy., 1888. US Patent 376588.

Gabrielli, G. and von Karman, . What price speed? Mechanical Engineering, pages 775-781, October 1950.

Garcia, Mariano; Chatterjee, Anindya; Ruina, Andy, and Coleman, Michael. The simplest walking model: Stability, complexity, and scaling. ASME Journal of Biomechanical Engineering, 120(2):281-288, 1998.

Geyer, Hartmut. Simple Models of Legged Locomotion based on Compliant Limb Behavior. $\mathrm{PhD}$ thesis, Friedrich Schiller University of Jena, 2005.

Gonzalez de Santos, Pablo; Garcia, Elena, and Estremera, Joaquin. Walking robots. Quadrupedal Locomotion: An Introduction to the Control of Four-legged Robots, pages 3-32, 2006.

Hildebrand, Milton. Symmetrical gaits of horses. Science, 150(3697):701-708, 1965.

Hirose, Shigeo; Yoneda, Kan, and Tsukagoshi, Hideyuki. TITAN VII: Quadruped walking and manipulating robot on a steep slope. In Robotics and Automation, 1997. Proceedings., 1997 IEEE International Conference on, volume 1, pages 494-500. IEEE, 1997. 
Hirukawa, Hirohisa; Kanehiro, Fumio; Kaneko, Kenji; Kajita, Shuuji; Fujiwara, Kiyoshi; Kawai, Yoshihiro; Tomita, Fumiaki; Hirai, Shigeoki; Tanie, Kazuo; Isozumi, Takakatsu; Akachi, Kazuhiko; Kawasaki, Toshikazu; Ota, Shigehiko; Yokoyama, Kazuhiko; Handa, Hiroyuki; Fukase, Yutaro; ichiro Maeda, Jun; Nakamura, Yoshihiko; Tachi, Susumu, and Inoue, Hirochika. Humanoid robotics platforms developed in HRP. Robotics and Autonomous Systems, 48(4):165-175, 2004. Humanoids 2003.

Hobbelen, Daan GE and Wisse, Martijn. Limit cycle walking, volume 14. Chapter, 2007.

Honda, . ASIMO - the honda humanoid robot. Technical report, Honda Motor Co., September 2007.

Ingram, A. J. Numerical kinematic and kinetic analysis of a new class of twelve bar linkage for walking machines. Master's thesis, Rand Afrikaans University, December 2004 .

Inman, Verne Thompson; Ralston, Henry James, and Todd, Frank. Human walking. Williams \& Wilkins, 1981.

Irawan, Addie and Nonami, Kenzo. Compliant walking control for hydraulic driven hexapod robot on rough terrain. Journal of Robotics and Mechatronics, 23(1):149, 2011.

Irawan, Addie; Nonami, Kenzo; Ohruko, Hiroshi; Akutsu, Yasunaga, and Imamura, Shota. Adaptive impedance control with compliant body balance for hydraulically driven hexapod robot. Journal of System Design and Dynamics, 5(5):893-908, 2011.

Jansen, Theo. Strandbeest. Website, 2014. http://www.strandbeest.com/.

JunBae Lee, JunHo Song and Chung, DaeWoong. Reference board for IKCS12F60AA. 2010.

Kajita, Shuuji and Espiau, Bernard. Legged robots. In Bruno Siciliano, Oussama Khatib, editor, Handbook of Robotics, pages 361-389. Springer, 2008.

Kajita, Shuuji; Tani, Kazuo, and Kobayashi, Akira. Dynamic walk control of a biped robot along the potential energy conserving orbit. In Proceedings of the IEEE International Workshop on Intelligent Robots and System, pages 789-794, 1990. 
Kajita, Shuuji; Kanehiro, Fumio; Kaneko, Kenji; Yokoi, Kazuhito, and Hirukawa, Hirohisa. The 3D linear inverted pendulum mode: A simple modeling for a biped walking pattern generation. In Intelligent Robots and Systems, 2001. Proceedings. 2001 IEEE/RSJ International Conference on, volume 1, pages 239-246. IEEE, 2001.

Karnopp, D. and Margolis, D. Analysis and simulation of planar mechanism systems using bond graphs. ASME Journal of Mechanical Design, 101:187-191, April 1979.

Karnopp, Dean C; Margolis, Donald L, and Rosenberg, Ronald C. System dynamics: a unified approach. John Wiley, 1990.

Kato, Keisuke and Hirose, Shigeo. Development of the quadruped walking robot, titanixmechanical design concept and application for the humanitarian de-mining robot. Advanced Robotics, 15(2):191-204, 2001.

Knight, Rob and Nehmzow, Ulrich. Walking robots - a survey and a research proposal. Technical Report CSM-375, University of Essex, October 2002.

Kunzig, Robert. The physics of walking. Discover, 22(7), July 2001. URL http:// discovermagazine.com/2001/jul/featphysics.

Kuo, Arthur D. The six determinants of gait and the inverted pendulum analogy: A dynamic walking perspective. Human Movement Science, 26(4):617 - 656, 2007.

Kuo, Arthur D.; Donelan, J. Maxwell, and Ruina, Andy. Energetic consequences of walking like an inverted pendulum: Step-to-step transitions. Exercise and Sport Sciences Reviews, 33(2):88-97, April 2005.

Lawyer, David S. Rail vs auto energy efficiency, July 2004. URL http://www . lafn.org/ $\sim$ dave/trans/energy/rail_vs_autoEE.html.

Lim, Hun-Ok and Takanishi, Atsuo. Biped walking robots created at waseda university: Wl and wabian family. Philosophical Transactions of the Royal Society A: Mathematical, Physical and Engineering Sciences, 365(1850):49-64, 2007.

Luo, Ren C; Sheng, Jun, and Chang, Peng-Hsi. Biped robot limit cycle walking generation considering energy dissipation caused by impact. In Advanced Robotics and Intelligent Systems (ARIS), 2013 International Conference on, pages 17-22. IEEE, 2013.

Mahon, J. J. Walking toy., 1914. US Patent 1007316, Serial Nos. 541801 and 796095. 
Margaria, Rudolfo. Biomechanics and Energetics of Muscular Exercise. Oxford University Press, 1976.

Martin-Alvarez, A.; de Peuter, W.; J.Hillebrand, ; P.Putz, ; A.Matthyssen, , and de Weerd, J.F. Walking robots for planetary exploration missions. In World Automation Congress, May 1996.

McGeer, Tad. Passive dynamic walking. The International Journal of Robotics Research, 9(2):62-82, April 1990a.

McGeer, Tad. Passive walking with knees. In IEEE International Conference on Robotics and Automation, pages 1640-1645, 1990b.

McMahon, Thomas A. Mechanics of locomotion. The International Journal of Robotics Research, 3(2):4-28, 1984a.

McMahon, Thomas A. Muscles, reflexes, and locomotion. Princeton University Press Princeton, 1984b.

Minetti, A.E. and Alexander, R.M. A theory of metabolic costs for bipedal gaits. Journal of Theoretical Biology, 186(4):467-476, 1997.

Mohan, N.; Undeland, T. M., and Robbins, W. P. Power electronics: converters, applications, and design, chapter 10. Wiley, New Delhi, India, 3rd ed. edition, 2005.

Morrey, J.M.; Lambrecht, B.; Horchler, Andrew D.; Ritzmann, Roy E., and Quinn, R.D. Highly mobile and robust small quadruped robots. In Intelligent Robots and Systems, 2003. (IROS 2003). Proceedings. 2003 IEEE/RSJ International Conference on, volume 1, pages 82-87 vol.1, Oct 2003.

Mosher, Ralph S. Exploring the potential of a quadruped. Technical report, SAE Technical Paper, 1969.

Murphy, Michael P; Saunders, Aaron; Moreira, Cassie; Rizzi, Alfred A, and Raibert, Marc. The littledog robot. The International Journal of Robotics Research, 2010.

Muybridge, Eadweard. Animals in motion. Courier Dover Publications, 1957.

Nagasaka, K.; Kuroki, Y.; Suzuki, S.; Itoh, Y., and Yamaguchi, J. Integrated motion control for walking, jumping and running on a small bipedal entertainment robot. In Robotics and Automation, 2004. Proceedings. ICRA '04. 2004 IEEE International Conference on, volume 4, pages 3189-3194 Vol.4, April 2004. 
Narukawa, T.; Yokoyama, K., and Takahashi, M. Design and stability analysis of a 3D rimless wheel with flat feet and ankle springs. Journal of System Design and Dynamics, 3(3):258-269, 2009.

Nonami, K. Development of mine detection robot comet-ii and comet-iii. In SICE 2002. Proceedings of the 41st SICE Annual Conference, volume 1, pages 346-351 vol.1, Aug 2002.

Patil, M. B.; Ramanarayanan, V., and Ranganathan, V. T. Simulation of power electronic circuits, chapter 14. Narosa, 2009.

Patnaik, Lalit. Vector control of single phase induction motor. Master's thesis, Indian Institute of Science, June 2009.

Pavei, Gaspare; Cazzola, Dario; La Torre, Antonio, and Minetti, Alberto E. The biomechanics of race walking: Literature overview and new insights. European Journal of Sport Science, 14(7):661-670, 2014.

Pratt, Jerry E. Exploiting inherent robustness and natural dynamics in the control of bipedal walking robots. PhD thesis, Massachusetts Institute of Technology, 2000.

Pratt, Jerry E. and Drakunov, Sergey V. Derivation and application of a conserved orbital energy for the inverted pendulum bipedal walking model. In Robotics and Automation, 2007 IEEE International Conference on, pages 4653-4660. IEEE, 2007.

Pratt, Jerry E. and Pratt, Gill. Exploiting natural dynamics in the control of a 3D bipedal walking simulation. In Proceedings of the International Conference on Climbing and Walking Robots (CLAWAR99), pages 1-11, 1999.

Quinn, R.D.; Offi, J.T.; Kingsley, D.A., and Ritzmann, Roy E. Improved mobility through abstracted biological principles. In Intelligent Robots and Systems, 2002. IEEE/RSJ International Conference on, volume 3, pages 2652-2657 vol.3, 2002.

Radtke, JL. The energetic performance of vehicles. Open Fuels 63 Energy Science Journal, $1: 11-18,2008$.

Raibert, Marc. Legged robots that balance, volume 3. MIT press Cambridge, MA, 1986a.

Raibert, Marc. Legged robots. Communications of the ACM, 29(6):499-514, June 1986b.

Robotics-Unlimited, . Meet OutRunner: The world's first remotely controlled running robot. YouTube, May 2014. URL https://www . youtube.com/watch?v=LTIpdtv_AK8. 
Sakagami, Y.; Watanabe, R.; Aoyama, C.; Matsunaga, S.; Higaki, N., and Fujimura, K. The intelligent ASIMO: system overview and integration. In Intelligent Robots and Systems, 2002. IEEE/RSJ International Conference on, volume 3, pages 2478-2483 vol.3, 2002.

Saranli, Uluc; Buehler, Martin, and Koditschek, Daniel E. RHex: A simple and highly mobile hexapod robot. The International Journal of Robotics Research, 20:616-631, 2001.

Smith, A.C. and Berkemeier, M.D. The motion of a finite-width rimless wheel in 3d. In Robotics and Automation, 1998. Proceedings. 1998 IEEE International Conference on, volume 3, pages 2345-2350 vol.3, 1998.

Srinivasan, Manoj. Fifteen observations on the structure of energy-minimizing gaits in many simple biped models. Journal of The Royal Society Interface, 8(54):74-98, 2011.

Strogatz, Steven H. Nonlinear dynamics and chaos: with applications to physics, biology and chemistry. Perseus publishing, 2001.

Taylor, Graham and Thomas, Adrian. Evolutionary Biomechanics. Oxford University Press, 2014.

Tucker, V. A. The energetic cost of moving about. American Scientist, 63(4):413-419, July-August 1975.

Tucker, V.A. Energetic cost of locomotion in animals. Comparative Biochemistry and Physiology, 34:841-846, 1970.

Umanand, L. Power electronics essentials and applications, chapter 5. Wiley, New Delhi, India, 1st ed. edition, 2009.

Usherwood, James R. Why not walk faster? Biology Letters, 1(3):338-341, 2005.

Usherwood, James R.; Szymanek, Katie L., and Daley, Monica A. Compass gait mechanics account for top walking speeds in ducks and humans. Journal of Experimental Biology, 211(23):3744-3749, 2008.

Usherwood, James R.; Channon, A.J.; Myatt, J.P.; Rankin, J.W., and Hubel, T.Y. The human foot and heel-sole-toe walking strategy: a mechanism enabling an inverted pendular gait with low isometric muscle force? Journal of The Royal Society Interface, 9(75):2396-2402, 2012. 
Vukobratovic, Miomir and Borovac, Branislav. Zero-moment point - thirty five years of its life. International Journal of Humanoid Robotics, 1(01):157-173, 2004.

Vukobratovic, Miomir and Juricic, Davor. Contribution to the synthesis of biped gait. Biomedical Engineering, IEEE Transactions on, (1):1-6, 1969.

Waldron, Kenneth J. and McGhee, Robert B. The adaptive suspension vehicle. IEEE Control Systems Magazine, 6(6):7-12, 1986.

Wilson, J. E. Walking toy, 1938. US Patent 2140275.

Wisse, Martijn and van Frankenhuyzen, Jan. Design and construction of MIKE; a 2D autonomous biped based on passive dynamic walking. In Kimura, Hiroshi; Tsuchiya, Kazuo; Ishiguro, Akio, and Witte, Hartmut, editors, Adaptive Motion of Animals and Machines, pages 143-154. Springer Tokyo, 2006.

Yan, J. and Agrawal, S. K. Rimless wheel with radially expanding spokes: dynamics, impact, and stable gait. In Proc. IEEE Int. Conf. Robot. Autom., April 2004. 
